\title{
RESULTS OF FIVE YEARS OF POTTERY ANALYSIS IN THE TEMPLE OF MILLIONS OF YEARS OF THUTMOSIS III IN WESTERN THEBES (2011-2015)
}

Bettina Bader', Myriam Seco Álvarez ${ }^{2}$

Abstract: This article discusses some of the ceramic finds from the Temple of Millions of Years of Thutmosis III in Thebes/West recorded from 2011 to 2015. Whilst the finds presented in this article mostly date from the late Middle Kingdom and Second Intermediate Period, tombs created before the temple was built, are discussed in full, selected New Kingdom pottery types are also shown, e.g. from a foundation deposit. An interesting cluster of tombs from approximately the $11^{\text {th }}$ Dynasty was situated to the north of the temple, some pottery of which is also published.

Keywords: Thebes, Pottery, late Middle Kingdom, Thutmosis III, New Kingdom, Temple of Millions of Years, foundation deposit

\section{1.) Introduction}

After analysing the ceramic material of the temple of millions of years of Thutmosis III for five seasons of 4 to 6 weeks duration each it seems time to present a more substantial report of the work achieved and the first results of the pottery analysis. This article will provide a comprehensive overview of the kind of results to be expected from work in a funerary temple of the $18^{\text {th }}$ Dynasty. ${ }^{3}$

Previous limited work was done by Abdallah Alaeldine in the short seasons of $2008^{4}$ and 2009, which will not be treated here.

\footnotetext{
Principal Investigator of the START Project Y754-G19 : Beyond Politics, Material Culture in Second Intermediate Period Egypt and Nubia, Institute of Oriental and European Archaeology, Austrian Academy of Sciences.

2 Director of the Thutmosis III Project.

3 Bettina Bader would like to thank E. Schuster, who inked most of the drawings shown in this article.

4 Seco Álvarez et al. 2010.

5 Seco Álvarez/Martinez Babon 2015b, 35-41.

6 These traces include disused 'Beer Bottles' filled with thick white plaster (lime and gypsum) as well as numerous sherds of clear early 18th Dynasty pottery with adhering pigments and mixtures thereof in yellow, blue, green and pink. Bettina Bader would like to thank Agustín Gamarra Campuzano for discussing pigments and simple methods of distinguishing lime from gypsum plaster. See also SECO
}

Very frequently the dating of the contexts, excavated between 2010 and 2015, relies entirely on the pottery analysis. In chronological order, the types of contexts with pottery located within, around and under the temple are as follows:

a) Middle Kingdom tombs ${ }^{5}$ of the early and the late Middle Kingdom within and without the temple area;

b) re-use of some of these tombs in the Second Intermediate Period or the early New Kingdom;

c) destruction or closure of the (robbed) tombs within the temple area during the construction of the temple;

d) deposits from founding the temple;

e) traces of building or repairing parts of the temple, but usually out of context; ${ }^{6}$

f) trash or levelling deposits to the north of the temple;

g) Ramesside use of parts of the temple, ${ }^{7}$ and perhaps also in the 'settlement structures' to the south outside the enclosure wall, which were not discovered before ${ }^{8}$

h) Late Period or Ptolemaic reuse of some of the tombs (just outside the temple);

i) Limited Late Antique activity in some parts of the temple and its environs (e.g. around the Pylon, some areas north of the southern enclosure wall) ${ }^{9}$

Álvarez et al. 2012/13 (appeared 2016), 358; BADER 2015, 305, cat.nos 22-23, fig. 4.22-23, for actual examples.

Seco Álvvarez/Martinez Babon 2015d, 383-391.

8 B. Bader would like to thank C. Knoblauch and D. Aston for revising the English of this article. Thanks go to D. Aston for his expertise of Ramesside and Late Period pottery and to V. Müller for some comments. Any remaining mistakes are her responsibility.

9 Whether the limited visibility of Late Antique material is grounded in the previous work of Grébaut (reported by Daressy), Weigall and Ricke, who may have removed much of such material or in actual limited activity is impossible to judge today. From experience is seems unlikely that any late ceramic material could have been so effectively removed. 
j) Ottoman to modern reuse of some parts of the temple (oven built within the eastern enclosure wall). ${ }^{10}$

So far there are no contexts from within the temple that could be assigned to actual cult activities because in most places the original temple floor level was not in place anymore and Grébaut, Weigall as well as Ricke already excavated in most parts within the temple, mainly in the magazines in the north and the south and the actual cult buildings. ${ }^{11}$ The current excavation proceeds until bedrock is reached, which is in most places well below the temple floor.

\section{2.) Processing method}

The processing method aims to record the type of material and its quantity for each find context before any reduction of the material is undertaken. ${ }^{12}$ To this end bespoke recording sheets were developed by Bettina Bader. These sheets simplify and speed up recording the original context in detail, including the body sherds and the diagnostics (or complete profiles of vessels) many of which are then documented by drawing in a subsequent step. The body sherds are divided by fabric, ware and vessel shape and subsequently counted as well as weighed, in order to obtain information for each context on the degree of brokenness of the material, which may vary from one context to another, ${ }^{13}$ and to assess the quantity of the single types by means of a constant measurement. ${ }^{14}$ Most of the body sherds, which cannot be used for reconstructing larger parts of vessels are then discarded except painted or otherwise decorated body sherds. Differences in treatment are made in contaminated surface contexts where only unusual pottery is kept or forms missing from the site corpus.

After the initial season 2011 it became clear that most of the excavated contexts contained a very large number of various versions of bases of the so-called New Kingdom 'Beer jars', Holthoer's class BB. ${ }^{15}$ Literally tens of 1000 s of bases and base fragments of these are found. They are very bulky and quite repetitive and for this reason their type, fabric, quantity, dimensions and state of preservation were recorded on specialised sheets developed by Bettina Bader. Usually it was not possible to reconstruct full profiles of broken BB jars from surface contexts due to the quantity of very similar looking sherds in terms of fabric and ware and wall thickness. The effort made to do so varies according to the quality of the context. However, these vessels often survive almost complete. After trials of reconstruction the recorded duplicates of the various types are discarded. For the variety of types and a preliminary quantification of one small sector see below 7.2.

\section{1.) Storage of ceramic material}

The bulk of the material has to be stored in the open air in the temple area with smaller volumes of materials stored in already excavated tombs (such as tombs $\mathrm{I}^{16}$ and $\mathrm{VII}^{17}$ ) and in one small site magazine for the most important material behind, i.e. west, of the temple proper. Storage in the open air makes use of wooden boxes made from palm tree sticks (garrith) which are well labelled and covered with straw mats for protection. For temporary storage this method is quite useful.

\section{3.) Fabric classification}

The fabric classification used for the ceramic material of this site is based on the Vienna System $^{18}$ with some adjustments to the local varieties of fabrics in Thebes and the chronological range. The classification is based on macroscopic examination of fresh sections (parallel to the rilling lines) of sherds by means of a $10 \times$ magnifying lens. Unfortunately a scientific examination of the

\footnotetext{
14 See Bader 2016; Bader 2010.

15 Holthoer 1977, pl. 18.

16 In square 1000-South1. See plan in Seco Álvarez et al. 2012/13 (appeared 2016), plan 1; Seco Álvarez et al. 2010, 30-31.

17 In square 960-970-980-South4. See plan Weigall 1907, 286. In Weigall's plan this tomb is located in his square D1.

18 Nordström/Bourriau 1993, 147-180.
} 
material by means of thin sections and petrography or chemical methods has not yet been possible. Nevertheless the data collected is presented here for a complete overview of the nature of the raw materials even though the photos are not ideal (Fig. 1).

Despite the fact that a number of different chronological phases are present in the pottery repertoire of the temple, it was decided to use the same fabric codes for the different chronological periods although there are differences between a 'Middle Kingdom Nile B2' and a 'New Kingdom Nile B2' especially in the way the material has been fired. As long as the 'ingredients' are the same, the same label is used. The firing technology is then noted separately (e. g. hard fired, 'sandwich', etc.).

There are regional differences in the various Nile silt fabric recipes, which are particularly obvious comparing the Nile clay fabrics found in the Delta, ${ }^{19}$ Memphis, Ehnasya el-Medina, Dayr al-Barsha and Thebes, even if only in a diachronic view. This fact provides vital evidence for a probably larger number of local pottery manufacturing workshops, although none of them has been discovered yet. This, on the other hand, is connected with the lagging behind of settlement archaeology in Egypt in general. At Thebes, and certainly not

Fig. 1 - Description

\begin{tabular}{|c|c|c|c|c|c|}
\hline Fig. & Fabric & Location & No. & Width of section & Drawing of vessels \\
\hline $1 . \mathrm{a}$ & Nile B1 & $\mathrm{R} 4 / 5-\mathrm{L} 11$ & -1 & $5 \mathrm{~mm}$ & hemi. cup \\
\hline $1 . \mathrm{b}$ & Nile B2/C1 & 900-N5-L4 & 8758 & $17 \mathrm{~mm}$ & 4.a \\
\hline 1.c & Nile C2 & Tomb V-L9 & -4 & $10 \mathrm{~mm}$ & $18 . \mathrm{g}$ \\
\hline $1 . d$ & Nile C2 & Tomb III-L10 & 9191 & $9 \mathrm{~mm}$ & $8 . h$ \\
\hline 1.e & Nile C3 & Tomb V-L7/8 & 9398 & $11 \mathrm{~mm}$ & 18.e \\
\hline 1.f & Nile C4 & Tomb II-L4 & -7 & $13 \mathrm{~mm}$ & 6.f \\
\hline $1 . \mathrm{g}$ & Marl A2 & Tomb V-L9 & $-14-15$ & $8 \mathrm{~mm}$ & 18.j \\
\hline $1 . \mathrm{h}$ & Marl A3 & Tomb IV-L2 & -10 & $7 \mathrm{~mm}$ & $15 . \mathrm{i}$ \\
\hline $1 . \mathrm{i}$ & Marl A4 & Tomb II-L4 & -1 & $8 \mathrm{~mm}$ & $6 . \mathrm{i}$ \\
\hline $1 . \mathrm{j}$ & Nile B2 & 910-N1-L11 & -31 & $5 \mathrm{~mm}$ & $42 . \mathrm{d}$ \\
\hline $1 . \mathrm{k}$ & Nile B2/C1 & 910-N1-L11 & -4 & $8 \mathrm{~mm}$ & $40 . \mathrm{e}$ \\
\hline 1.1 & Nile B3 & 950-N5-L3 & 8723 & $75 \mathrm{~mm}$ & Bader 2015, fig. 5.24 \\
\hline $1 . \mathrm{m}$ & Nile B4 & 920-S5-L4 & -99 & $11 \mathrm{~mm}$ & $\mathrm{BB}$ jar base \\
\hline $1 . n$ & Nile D & 930-940-N4-Sd & -46 & $7 \mathrm{~mm}$ & $\mathrm{BB}$ jar rim \\
\hline 1.0 & Marl A2 & EWN-Sd & -2 & $5 \mathrm{~mm}$ & jar \\
\hline 1.p & Marl A4 & 910-N1-L6 & -20 & $5 \mathrm{~mm}$ & $42 . \mathrm{j}$ \\
\hline $1 . q$ & Marl A4 & 900-S2-L1 & 8371 -Painted b.s. & $10 \mathrm{~mm}$ & - \\
\hline $1 . r$ & Marl B & 910-N1-L11 & Body sherd & $9 \mathrm{~mm}$ & - \\
\hline $1 . \mathrm{s}$ & Marl D & 940-S3-L1 & Body sherd & $10 \mathrm{~mm}$ & - \\
\hline $1 . t$ & FF 1 & 910-N1-L9 & -56 & $8 \mathrm{~mm}$ & $43 . b$ \\
\hline $1 . \mathrm{u}$ & FF 2 & 910-N1-L6 & Body sherd & $7 \mathrm{~mm}$ & - \\
\hline $1 . v$ & FF 3 & 930-S4-L2 & -1 & $5 \mathrm{~mm}$ & $53 . \mathrm{c}$ \\
\hline $1 . \mathrm{w}$ & FF 4 & 920-S2-L1 & Body sherd & $5 \mathrm{~mm}$ & - \\
\hline $1 . x$ & FF 4 & 930-940-N5-Sd & -3 & $9 \mathrm{~mm}$ & $53 . b$ \\
\hline 1.y & FF 5 & 920-S2-L1 & Body sherd & $8 \mathrm{~mm}$ & - \\
\hline $1 . z$ & FF 6 & 910-N1-L9 & Body sherd & $9 \mathrm{~mm}$ & - \\
\hline 1.aa & FF 7 & $\mathrm{R} 4 / 5-\mathrm{L} 13$ & -36 & $8 \mathrm{~mm}$ & $38 . c$ \\
\hline
\end{tabular}

19 See also Hein 2001, 143, for a sub-division of the New Kingdom Nile silts at the site of Ezbet Helmi. It could perhaps be hypothesised that in the New Kingdom more limestone particles are added to the pastes than before all over Egypt because also at Ezbet Helmi several limestone con- taining fabric variants are introduced in this period although no quantification is provided. At Thebes, in contrast, already the Middle Kingdom silt fabric material contains on average more limestone than for example that of Tell el-Daba. 


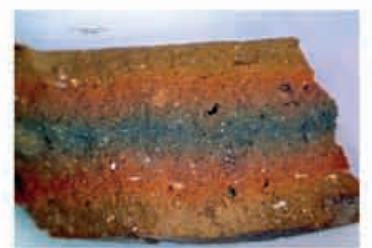

a)

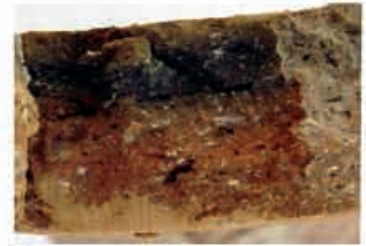

e)
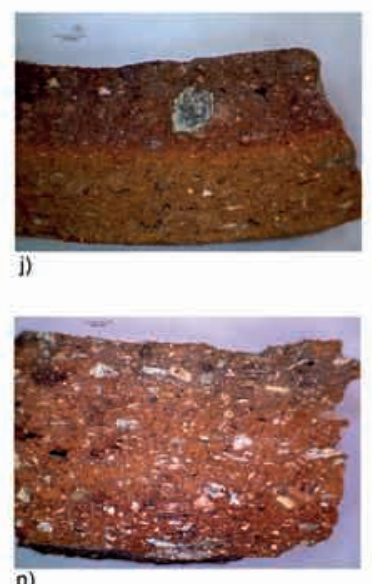

n)
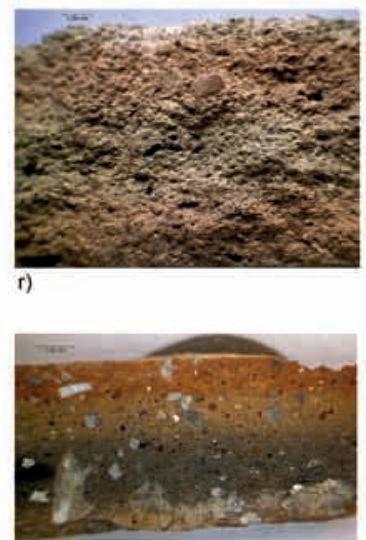

v)

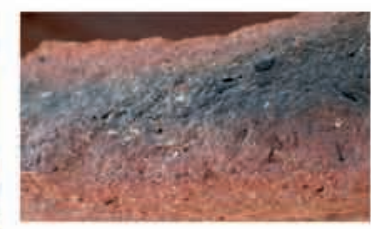

b)

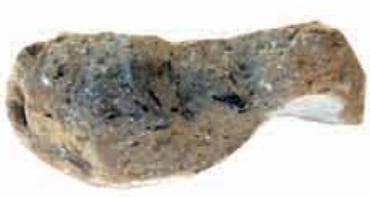

f)

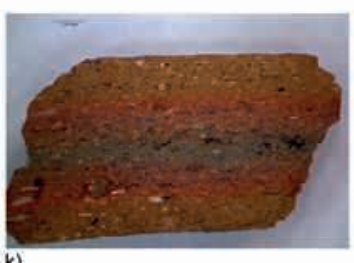

k)

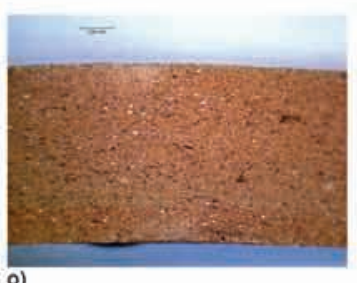

o)

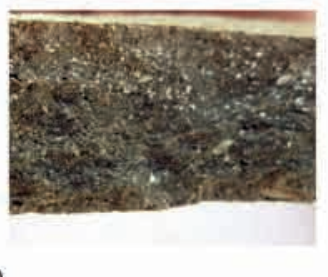

s)

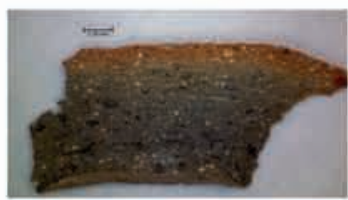

w)

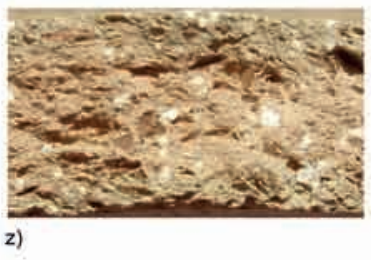

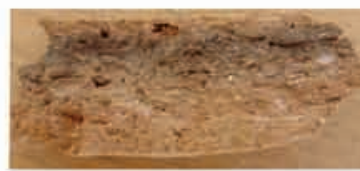

c)

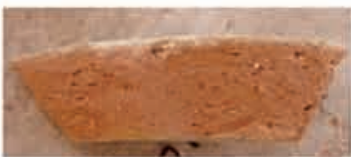

g)

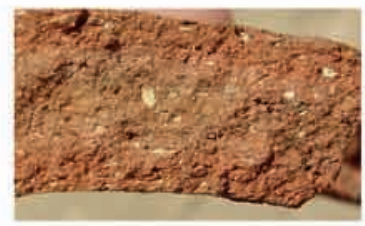

1)

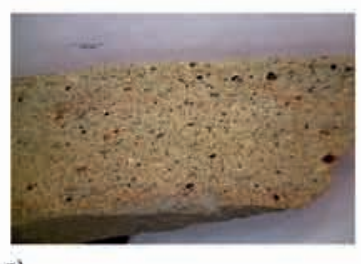

p)

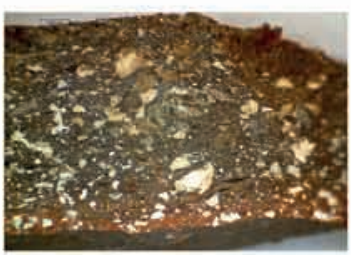

t)

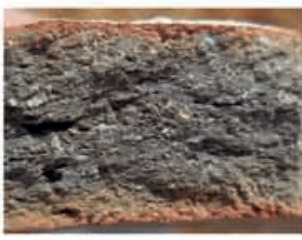

x)

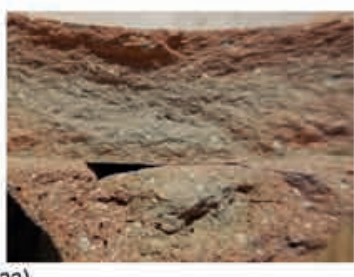

y)
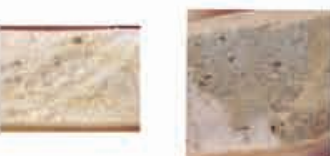

h)

i)

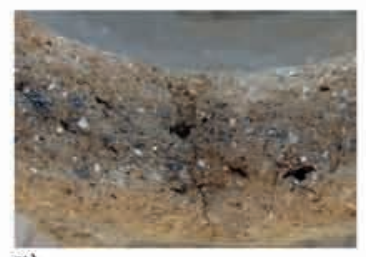

m)

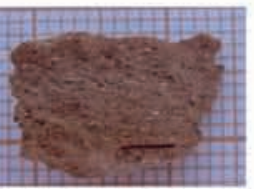

q)

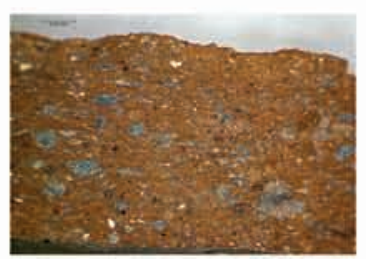

u)

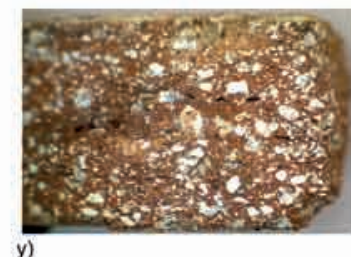

Fig. 1 Section photographs of Middle and New Kingdom Pottery fabrics, photos B. Bader. 
only at the temple of millions of years of Thutmosis III, ${ }^{20}$ the Nile silt fabrics in general contain more limestone inclusions than, for example, ceramic material derived from the north eastern Delta. This fact has been acknowledged by a modification of the classification system by adding ' 3 ' to the respective fabric, i.e. Nile B3; Nile C3. The differentiation to Nile $\mathrm{D}$ depends on the frequency of the limestone inclusions. Another seemingly special recipe not met in most of the other compared sites is a very special ware based on the Nile B2 description except that it is always uncoated, soft, and of a dull brown surface colour (the Munsell colours vary from 7.5YR5/3 brown, 7.5YR6/4 light brown to $10 \mathrm{YR} 7 / 3$ very pale brown). During processing these fragments are very distinctive and easy to recognise (see Fig. 2.a). The surface of Nile B4 may show traces of having been wet smoothed. The definition of wet smoothed here is the smoothing of the wet vessel on the wheel with the wet hands of the potter with the same basic material of which the vessel is being made. The most characterising feature, though, is the presence of inclusions which look like charred black plant remains of medium size $\left(2-5 \mathrm{~mm}^{21}\right)$. They are most obvious in the thickest parts of a vessel and may not be visible or present in the thinner parts of a vessel. Bettina Bader has seen one similar (accidential?) example of this ware in Ehnasya el Medina recently, but in a vessel type of very much earlier date. ${ }^{22}$

In the course of the work it became clear that Nile B4 is occasionally used for a range of other New Kingdom forms such as dishes, small beaker jars, medium to large jars and even 'flower pots'. A coarser variant in combination with Nile $\mathrm{C}$ was used in the Middle Kingdom (see below). Perhaps it is possible to identify a specific workshop in operation here.

\section{1.) The Middle Kingdom}

In the Middle Kingdom pottery repertoire the most common fabrics appearing are Nile B1, Nile B2, Nile B2/C1 (for the rougher variant of Nile

\footnotetext{
Aston 2008, 34.

21 This classification is based on the fabric recording sheets developed by J. Bourriau. Bourriau/Gallorini 2016, 23, fig. 3.

22 N. Ayers kindly told B. Bader that such a ware is also present at Edfu. Personal communication July 2014.
}

B2), Nile B3, Nile $\mathrm{C}(2)$, Nile $\mathrm{C} 3$, Nile $\mathrm{C}^{23}{ }^{23}$, and Marl A2, A3, A4 and Marl C1. Hitherto no imported fabrics from the Middle Bronze Age Levant or Nubia were encountered. A detailed description of the single fabrics will not be given in this instance but some preliminary colour photographs are provided in Fig. 1.a-i.

\section{2.) The New Kingdom}

In the early New Kingdom contexts the number of different Egyptian fabrics diminish somewhat, so that only Nile B2, Nile B2/C1 (for the rougher variant of Nile B2), Nile B3, Nile B4 and some rare varieties of Nile $C$ always in a hard fired version are met. The variety of Egyptian Marl clays changes to Marl A2, A4, B (Fig. 1.j-s) and what looks like an Egyptian mixed clay used especially for some of the pink coated red and black painted material.

A variety of imported fabrics from Syria-Palestine occur in in the New Kingdom, ${ }^{24}$ which seem to correspond in part to those from Tell el-Daba and Memphis. With the published material from Karnak North there is not much similarity. ${ }^{25}$ So far not many diagnostics of amphorae, which is the only imported vessel type encountered so far, were found in the course of the work and thus, the incipient classification system below is - far from ideal - also based on body sherds. The range of fabrics will need to be tested, extended and described in greater detail in the future. Also the dating needs to be further refined because some of the samples came from surface contexts.

FF-1 (Fig. 1.t) is an amphora handle from the foundation deposit 910-N1-L9-56 (Fig. 43.b). The exterior surface has no scum and the interior has a sort of blistered look with tiny holes. The section is dominated by abundant large white angular particles (probably limestone) of medium to large size. Grey particles are also visible in about the same size. The break is very dense, and there seems to be almost no space between the particles. It does resemble visually P90 of Memphis in certain respects such as the abundance of white inclu-

\footnotetext{
23 This fabric/ware type is much rarer in the Middle Kingdom, but the evidence is unequivocal because one MKbeer jar rim was made from a similar coarser fabric.

24 The fabrics certainly differ from Middle Bronze Age ones, encountered by Bettina Bader at sites like Tell el-Daba and Memphis/Kom Rabia.

25 HeIn 2012, pl. XIX.
} 
sions, but there it is burnt out limestone. It is certainly reminiscent of the IV-1 family of the Middle Bronze Age at Tell el-Daba but not the same. Perhaps it is related to one of D. Aston's Tell el-Daba $\mathrm{H} / \mathrm{VI}$ corpus. ${ }^{26}$

FF-2 (Fig. 1.u) is a fabric with uniform brownish red section and the dominating inclusion consists of abundant grey angular particles, some quartz inclusions and some limestone inclusions. There are also orange coloured inclusions as well as red argillaceous inclusion. The sample 910-N1L6 (foundation deposit) is from a body sherd. This fabric may be similar to one of D. Aston's Tell elDaba H/VI corpus. ${ }^{27}$

FF-3 (Fig. 1.v) shows a steel grey section with light yellowish brown and orange oxidation zones, whilst the grey core is located towards the interior of the vessel wall. The texture of the matrix is dense with very few air holes in it. The inclusions consists of grey rounded medium to small sized particles in ample spacing, dark red rounded particles are present as well as medium sized limestone particles and some quartz. The sample is from 930-S4-L2-1 (Fig. 53.c).

FF-4 (Fig. 1.w) consists of sherds with a wide grey core and very thin reddish oxidation zones. The inclusions comprise abundant quartz of various sizes and some limestone of small size in small quantities. The sample is from a body sherd in a surface context (920-S2-L1).

Perhaps the same fabric group is represented in the rim fragment of an amphora with a thick white scum (Fig. 1.x). Unfortunately, the sherd came from the dumping/levelling layer north of the temple. The sample is from sherd 930-940-N5-Sd-3 (Fig. 53.b).

FF-5 (Fig. 1.y) seems to be similar to FF-1 except that the firing of the vessel resulted in a uniform red section and air holes and other gaps in the matrix. The dominant inclusion is again abundant white to light grey angular particles from small to large size and some red argillaceous inclusions. The sample was taken from a body sherd from surface context 920-S2-L1. a)
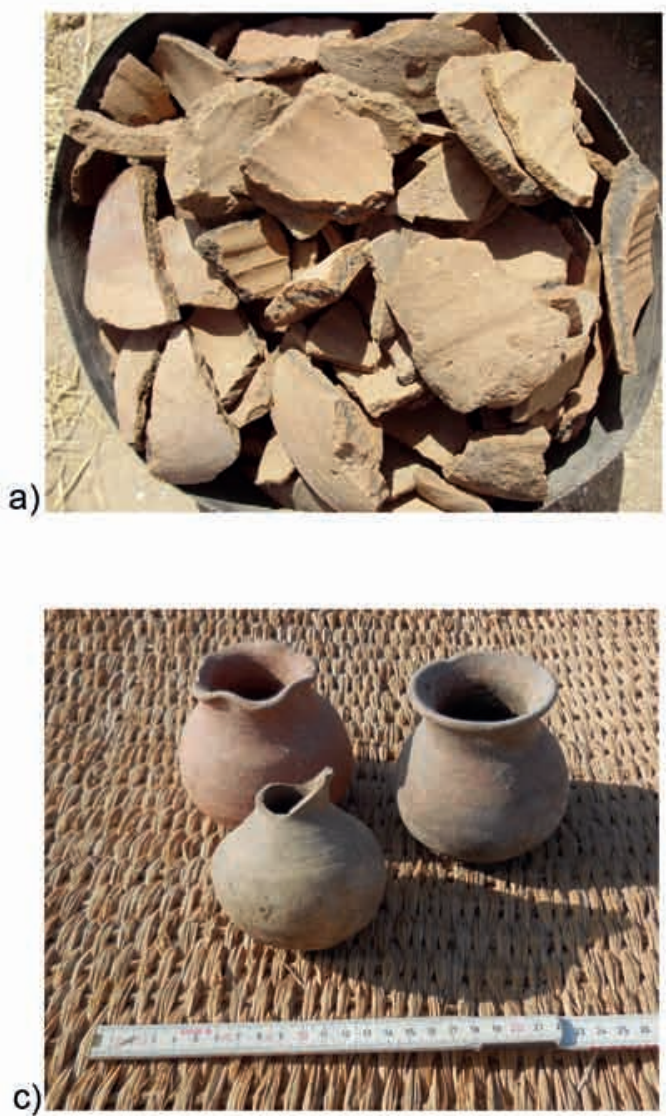

b)
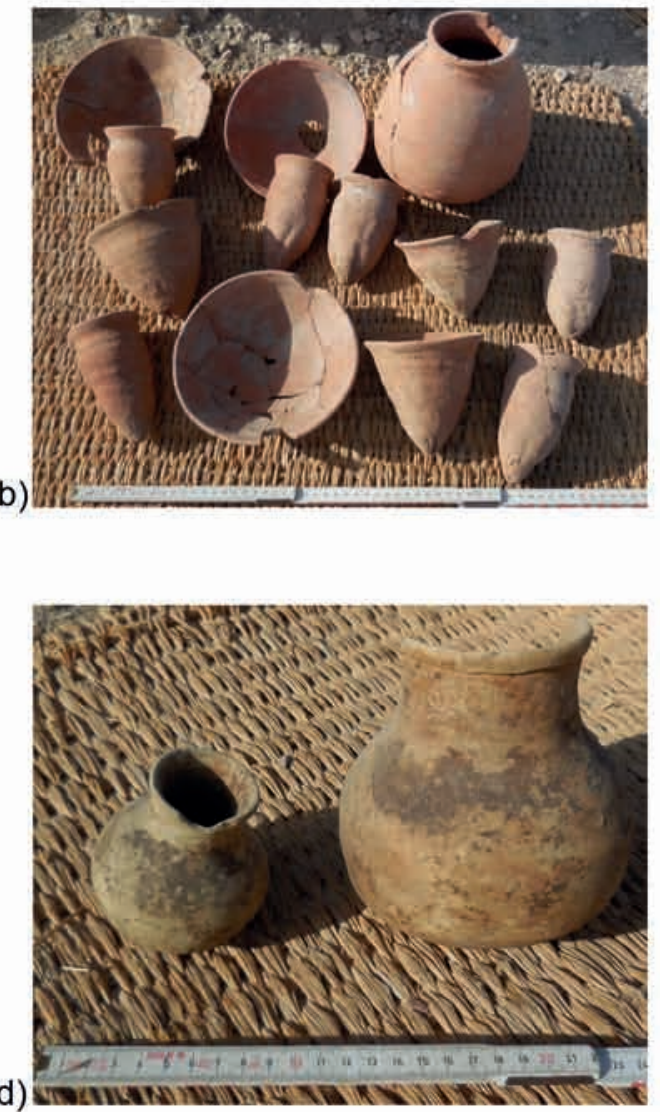

Fig. 2 a) Ware Nile B4. b) Pottery from 900-N5-L4. c) Pottery from 900-N5-L7. d) Pottery from 900-N5-L9. Photos by B. Bader. 
FF-6 (Fig. 1.z) has a uniform light brown to beige section with a diffuse darker layer on the exterior of the vessel wall. Whilst the fabric is dense, there are rounded white particles which commonly occur, some reddish brown shale particles, argillaceous inclusions and some fine quartz. The sample is from 910-N1-L9, foundation deposit, body sherd.

FF-7 (Fig. 1.aa) is derived from an amphora handle from tomb XI. This is a fine fabric with a diffuse light grey core and reddish beige oxidation zones. The inclusions are finely distributed and rather fine. Some limestone may be of medium size. Quartz occurs but in rather small quantities. The sample is from R 4/5-L13-36 (Fig. 38.c).

For the later New Kingdom the fabrics occurring represent the usual range of Nile and Marl clay fabrics and in addition Marl D in contexts dated to the Ramesside and even late Ramesside period (see below). Hitherto it has not been observed in more secure early New Kingdom contexts at the temple, which may corroborate the opinion that this fabric begins to appear in larger numbers only from the mid- $18^{\text {th }}$ Dynasty onwards.

\section{4.) Manufacturing technology of pottery and dating}

Due to the fact that the area on which the temple of Thutmosis III was erected was used already before as well as after the reign of this king, the different manufacturing technologies of the vessels provide an important additional dating criterion, ${ }^{28}$ especially when stratigraphic information is not available.

Obvious combination technologies like coiling and turning on a slowly rotating wheel/turning device $^{29}$ (with rather erratic and uneven rilling lines) suggest a date well before the New Kingdom. Also handmade bases (unevenly modelled/ pinched and hollowed ${ }^{30}$ or just squeezed together as in pointed beakers) or vertical trimming/scraping of various degrees of roughness on the exterior suggest a date in the early Middle Kingdom and earlier. A decisive criterion can be also seen in the orientation and ordering of organic particles within the paste. When the chaff and straw inclusions are not ordered parallel to the edge of the sherds it

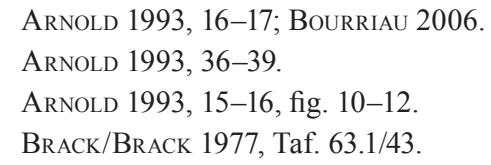

is clear that no centrifugal force was present to have an impact on these particles. In most cases of closed vessels this would mean that they belonged to an earlier period of pottery development. There are exceptions to this rule, of course, because even in the New Kingdom bread moulds are formed over a core and therefore show the same feature.

Mould made bases with added coils that were roughly smoothed and turned rims would suggest a rather earlier date than the New Kingdom again with one exception: the New Kingdom large storage jar type (with a white slip) retained this technology. ${ }^{31}$

Bases cut from the hump on the wheel/turning device with a string/wire show very particular traces that can easily be recognised ${ }^{32}$ but this technology was used for a long time in most chronological periods in Egypt. Therefore it is often difficult to distinguish pottery, for example model dishes, on the grounds of technology alone. Here the combination of various factors needs to be considered such as surface treatment, fabric and firing.

During the early New Kingdom the entirely wheel produced pottery increased ${ }^{33}$ and only the medium to large vessels were produced in several parts. Even BB jars are usually produced in two parts with the joining line in mid-height, frequently not very well smoothed so that the production process remains traceable.

An almost certain sign of early New Kingdom technology is visible in scrutinising the bases of slender elongated jars typical for the early $18^{\text {th }}$ Dynasty (Fig. 13.g): in the lowest third to quarter of the height of these vessels deep horizontal rillings spaced tightly together go down towards the base. This feature exists due to the fact that the dried vessel was put on the wheel (upside down) probably turned by an assistant to the potter and the potter used a wooden knife or similar tool to remove the excess clay and shape the base. In this procedure quartz grains and smaller stones were dragged horizontally across the vessel and produced these deep regular rillings. ${ }^{34}$

Similar signs have been observed in the manufacture of dishes and bowls contemporaneous with the reign of Thutmosis III, which were placed upside down on the wheel after drying and simi-

\footnotetext{
ARnold 1993, 54, fig. 60A-B.

ARnold 1976; Arnold 1993.

34 See Discussion by ArNold 1976, 29 pl. 7 for a photo of an example.
} 
larly trimmed with a tool, turning the wheel rather than the pot.

The kick wheel came into operation some time later ${ }^{35}$ and at the temple site there are hitherto only a few examples for such advanced manufacturing technology. They are very well recognisable among the pottery vessels produced with less innovative technology and they belong invariably to much later periods by analogy.

\section{5.) The pottery corpora from the temple and its environs}

\section{1.) The early Middle Kingdom tombs to the north of the enclosure wall}

Several simple tombs were situated close to the north eastern corner of the northern temple enclosure wall. The individuals were buried in rectangular wooden coffins, much deteriorated, with a few other grave gifts such as strings of beads or wooden bracelets. Seven individuals have been found to date with pottery, women, men and children. Interestingly the orientation of the bodies was not uniform, in that some of them were oriented east to west and others north to south. They may have belonged to a smaller cemetery. ${ }^{36}$ It seems to have been similarly varied as in Petrie's cemeteries A and B in el-Tarif, which he described as oriented "north of east" and "south of east". The structure and architecture of the Tarif tombs, though, was more varied. ${ }^{37}$ Parallels cited here exclusively refer to ceramic material from the Theban necropolis in order to try and get a clearer idea about the chronological position of this find. Parallels from other regions such as $\mathrm{Aswan}^{38}$ might only serve to obscure the chronological picture.

\subsection{1.) In situ finds with individuals: Burials} 900-North 5-L4, L7 and L9

A burial in square 900 -North5 with no L4 contained in addition to the body 13 intact or almost intact pottery vessels with archive numbers 8746 to 8758 (Fig. 2.b, 3, 4.a). They were arranged around the head of the deceased.

\footnotetext{
35 ARnold 1993, 16-17, 79-83, ca from the Third Intermediate Period onwards. KLotz 2013: at least since the reign of Ramesses II. B. Bader owes this reference to an anonymous reviewer.

36 Cf. Seco Álvarez et al. 2012/13 (appeared 2016), plan 1. Seco Álvarez/Martinez Babon 2015b, 36-37.
}

\section{Nile B2/C1 - Dishes (Fig. 3.a-c)}

Two dishes were found, which are very reminiscent of a late development of Old Kingdom Maidum bowls with the slightest remnant of a carination reminiscent of shapes of the Old Kingdom very high up the height of the vessels. The third dish (8756) shows an everted direct rim with a typically hand scraped base (cf. el Tarif cemeteries $^{39}$ ). Note that two of the dishes had an intentionally broken quite large hole in the base (no 8755 and 8757) and a pouring spout in the rim (no. 8755). The breaks were old, very carefully executed and smoothed. For these vessels no immediate parallels from Thebes are known to us.

No. 8755 , rd. $=17.8-18.0$, ht. $=6.5$, Nile B2/C1 + lime, section uniform light brown, surface red slipped on interior with red rim band (10R5/6 red) on exterior on uncoated (7.5YR6/4 light brown), coiled and turned on device, base coiled and subsequently scraped.

No. 8756 , rd. $=20.2$, ht. $=5.8$, Nile B3 soft, section uniform light brown, surface red slipped on interior (5YR6/6 reddish yellow) uncoated outside (7.5YR6/4 light brown), coiled and turned on device, base coiled and subsequently scraped.

No. 8757 , rd. $=16.6-16.8$, ht. $=6.9$, Nile B2/C1 + lime, section: pink core with red and brown oxidation zones, surface red slipped on interior with wide red rim band (10R5/6 red) on exterior on uncoated (2.5YR5/6 red), hand coiled and turned on turning device, base coiled and subsequently scraped.

\section{Nile B2/C1 - Pointed beakers (Fig. 3.d-I)}

Nine of these vessels belong to a type of pointed beaker that was very roughly made by hand: first by hollowing and pinching until some turning movement was exerted on these vessels and their rims finished by turning in some way because the horizontal rilling lines are very well visible. It remains unclear if the turning was done inside a bowl using it as turning device or if there was a turning device involved at all. At some stage the vessel was squeezed by hand and turned at the same time in some parts as becomes obvious by

\footnotetext{
Petrie 1909, 2.

Seidlmayer 2005.

39 Petrie 1909, pl. XIV. 32, 34; Arnold 1968, Abb. 3-4. It is clear that such a simple form finds parallels very easily, although the base technology is very distinctive.
} 

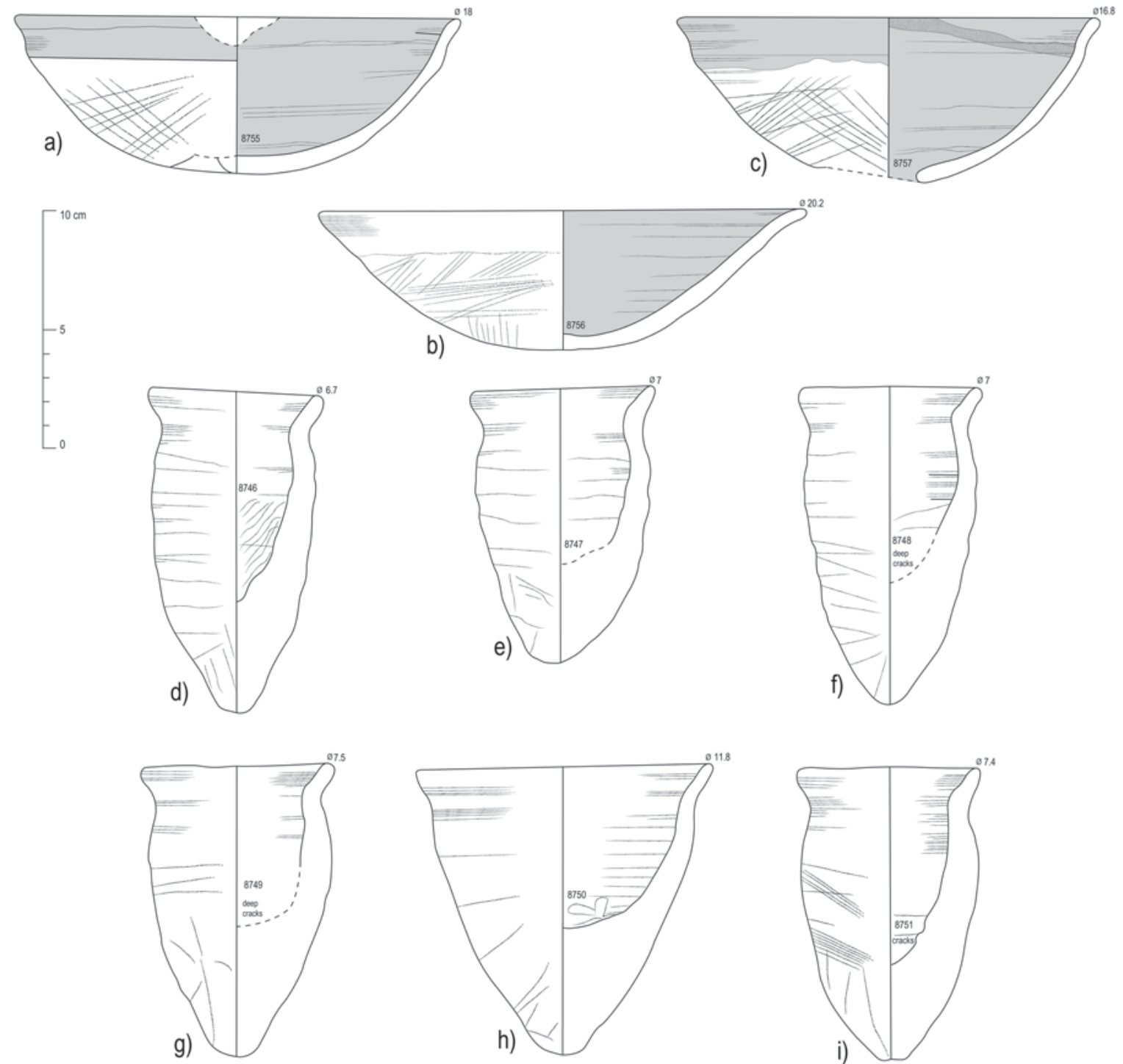

e)

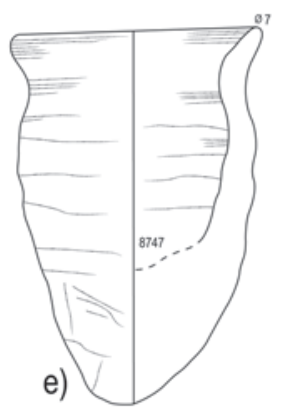

h)
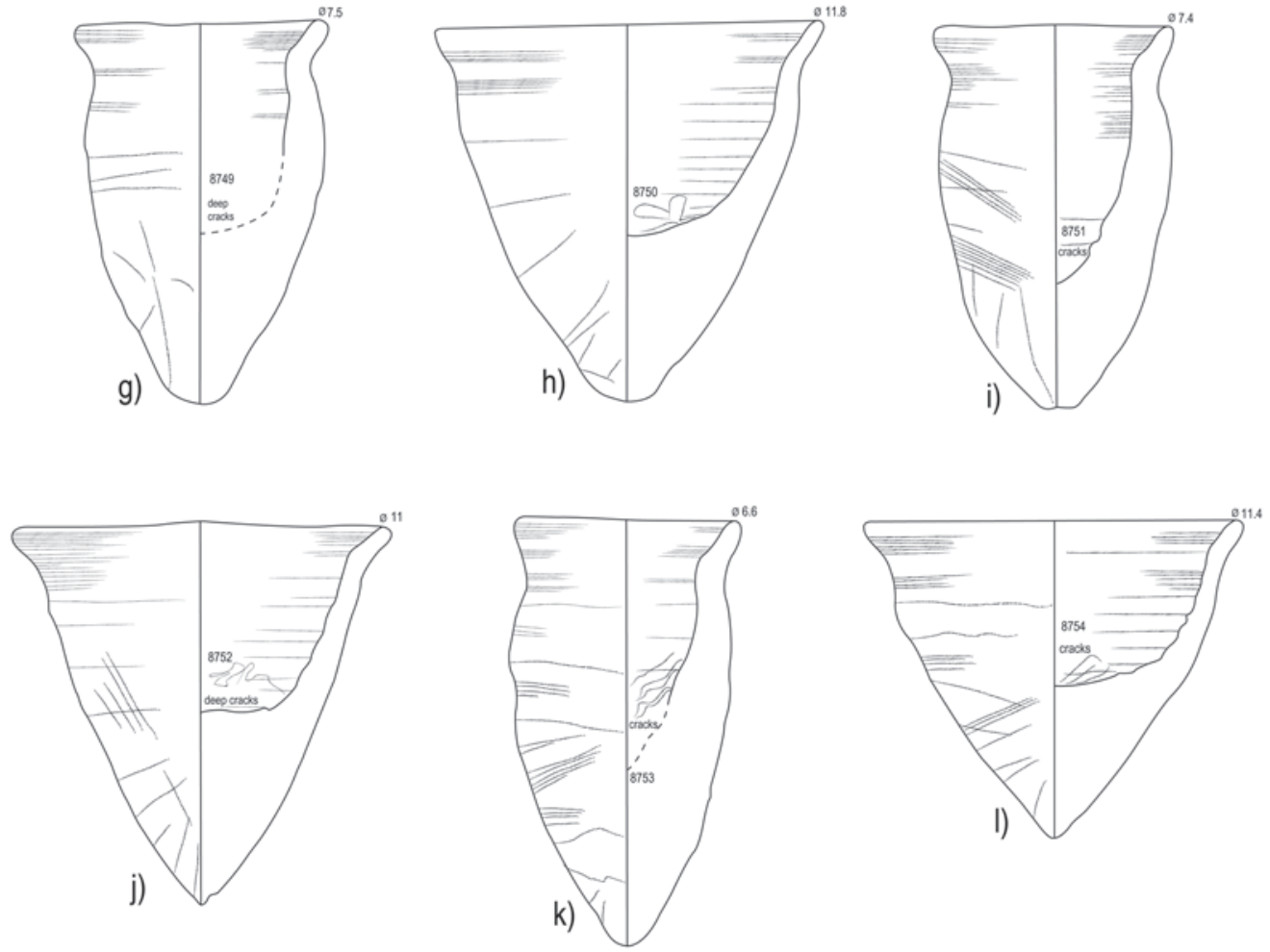

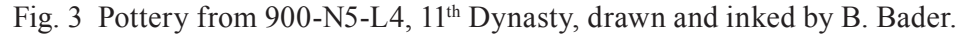


the turned folds visible on the interior. ${ }^{40}$ The width of the beakers varies and good parallels for the wider beakers no 8752 and no 8754 can be found in the debris of the royal tombs of the early $11^{\text {th }}$ Dynasty at el-Tarif. ${ }^{41}$ Similarly, more parallels can be found in Petrie's types 174-202 in cemeteries A and $\mathrm{B}$ at el-Tarif. ${ }^{42}$ In Seidlmayer's seriation the beakers (his type QA130, which includes wide and narrow shapes) appear in all his phases from I to III, but more frequently early in the sequence, which he dated to the $11^{\text {th }}$ Dynasty. ${ }^{43}$ In the area of the mortuary temple of Sethos I a Middle Kingdom shaft was found, which also contained such beakers. ${ }^{44}$ The Middle Kingdom Handbook positions these beakers, which are clearly to be distinguished from the later types (cf. Fig. 8.g, 10.b), early in the reign of Amenemhat I. The later examples show longer and more pinched 'handles' as well as a longer turned 'beaker' part. Finally the rim is always quite thick and direct without the slightly modelled rim turning outwards. ${ }^{45}$

No. 8746, rd.=6.3-6.7, ht. $=13.5$, Nile B2/C1 + lime, section: pink/red core with brown oxidation zones, surface uncoated (5YR6-7/6 reddish yellow), hand coiled and turned on turning device, base hand modelled, complete, weight $296 \mathrm{~g}$.

No. 8747, rd.=6.6-7.0, ht. $=11.5$, Nile B2/C1 + lime, section not visible, surface uncoated (7.5YR7/6 reddish yellow), hand coiled and turned on turning device, base hand modelled, complete, weight $284 \mathrm{~g}$.

No. 8748, rd. $=6.6-7.0, \mathrm{ht} .=11.2$, Nile $\mathrm{B} 2 / \mathrm{Cl}$, section: $\mathrm{red} / \mathrm{pink}$ core with reddish yellow oxidation zones, surface uncoated (5YR6/6 reddish yellow), hand coiled and turned on turning device, base hand modelled, complete, weight $281 \mathrm{~g}$.

No. 8749 , rd. $=7.1-7.5$, ht. $=12.1$, Nile B2/C1, section not visible, surface uncoated (5YR6/6 reddish yellow), hand coiled and turned on turning device, base hand modelled, complete, weight $253 \mathrm{~g}$.

No. 8750, rd. $=11.8-9.0$, ht. $=12.0$, Nile B2/C1, section: red/pink core with yellowish red oxidation zones, surface uncoated (5YR5/6 yellowish red),

40 Cf. Arnold 1976, 22, pl. 6.d.

41 ARnOld 1968, 50, Abb. 6.47, although this material is from the surface. It is derived from one chamber of the Tomb of Intef $N h t-n b-t p-n f r$, the saff el Dawaba. See also Arnold 1972, Abb. 2.5.1402.

42 Petrie 1909, pl. XV.

43 Seidlmayer 1990, 78-80, 104, 395.

44 Myśliwiec 1987, 29-30, 21a-22a; Schiestl/Seiler 2012, 1024-1025. hand coiled and turned on turning device, base hand modelled, complete, weight $432 \mathrm{~g}$.

No. 8751, rd.=6.8-7.4, ht. $=12.3$, Nile B2/C1 + some lime, section not visible, surface uncoated (2.5YR6/6 red), hand coiled and turned on turning device, base hand modelled, complete, weight $350 \mathrm{~g}$.

No. 8752, rd.= 11.0, ht. $=12.2$, Nile B2/C1 + lime, section grey core and violet, red and yellowish oxidation zones, surface uncoated (5YR6/6 reddish yellow), hand coiled and turned on turning device, base hand modelled, complete, weight $369 \mathrm{~g}$, two kiln stains ca 3.0 to $3.5 \mathrm{~cm}$ below the rim on ,south" and ,east".

No. 8753 , rd. $=6.3-6.6$, ht. $=13.6$, Nile B2/C1, section red core with brown oxidation zones, surface uncoated (5YR6/6 reddish yellow), hand coiled and turned on turning device, base hand modelled, intact except for chip on rim, weight $312 \mathrm{~g}$.

No. 8754 , rd. $=11.4$, ht. $=10.2$, Nile B2/C1 + lime, section: black core with violet and red oxidation zones, surface uncoated (7.5YR6/4 light brown), hand coiled and turned on turning device, base hand modelled.

\section{Nile B2/C1 - Medium squat jar (Fig. 4.a)}

This type of medium jar with short but visible neck and squat body shape again suggests parallels at el-Tarif from Petrie's (the best parallel is type $453^{46}$ ) as well as Arnold's work. ${ }^{47}$ It seems as if this general shape is best represented in Seidlmayer's work by his type QA215, which appears in the seriation in his Phases I and II, representing the entire $11^{\text {th }}$ Dynasty. ${ }^{48}$ Interestingly such shapes do not appear in Seiler's article on the Theban necropolis or in the entire Middle Kingdom Handbook.

No. 8758, rd. $=9.5$, max.d. $=17.8$, pres.ht. $=20.6$, Nile B2/C1 + lime, section black core with violet, red and reddish yellow oxidation zones, surface uncoated (2.5YR6/6 red), coiled and turned on device, base coiled and subsequently scraped, body visibly joined on, almost complete profile; In

\footnotetext{
Cf. Seiler 2012, 304, fig. 4.1, phase II.1.

Petrie 1909, pl. XVIII.

47 Arnold 1972, Abb. 5, no 10, I 1145. The earlier work published 1968 shows the manufacturing technology used, which resembles that described here very closely, although the body shapes differ slightly. Cf. ArNold 1968, Abb. 7. 64-66.

48 Seidlmayer 1990, 84-87, 395.
} 

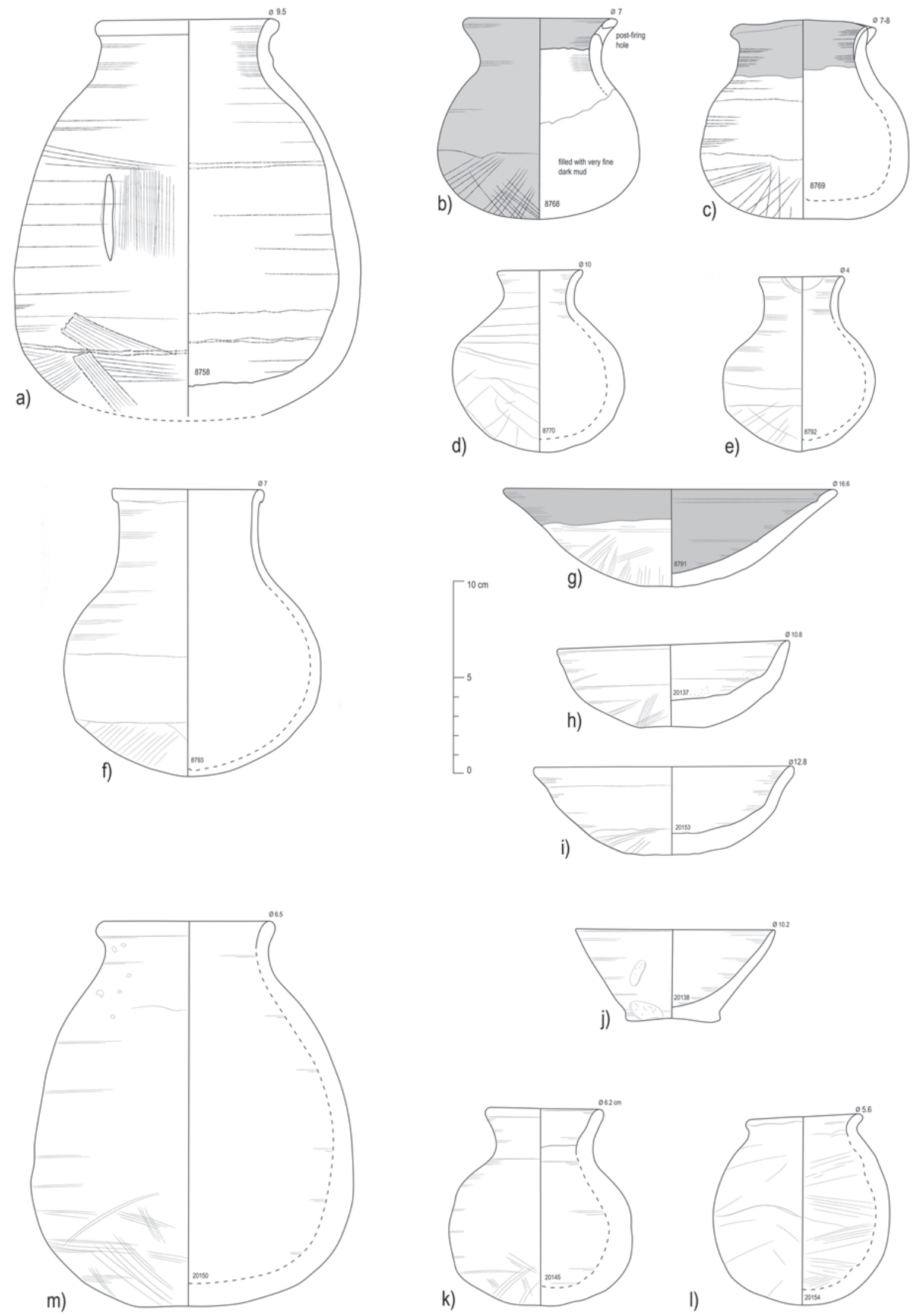

Fig. 4 a) Pottery from 900-N5-L4. b-d) Pottery from 900-N5-L7. e-f) Pottery from 900-N5-L9. g-1) Pottery from radîm

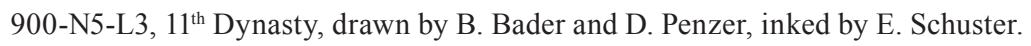


2015 a sherd was joined to the base from context 910N5L7. On the exterior in mid height there is a wide and deep pre-firing incision, rough manufacture. The section is shown in Fig. 1.b, the wall thickness is $1.7 \mathrm{~cm}$.

An in situ burial in the same square 900-North 5 was given number L7 and contained three almost intact pottery vessels of closed shape (nos 87688770) (Fig. 2.c) situated at the foot end of the coffin. With this burial there were no pointed handmade beakers.

\section{Nile B2/B3 - Small globular jar with everted rim (Fig. 4.b)}

This small jar with direct everted rim shows a slightly flattened base and a distinctive technology where at first the vessel was coiled and then turned on a centred (?) device. Finally, the base was trimmed with a tool with not very much smoothing done afterwards. In the rim there was a postfiring hole, perhaps for fastening some cover. The neck is relatively wide. This vessel shape is quite common in early Middle Kingdom Thebes and appears in Tarif (e.g type Petrie's $380^{49}$ ). In Seidlmayer's seriation this type falls within his QA200, which occurs very rarely and is evenly distributed over all his phases from the $11^{\text {th }}$ Dynasty to the reign of Senwosret II..$^{50}$

No. 8768 , rd. $=7.0$, max.d. $=10.3$, ht. $=10.3$, Nile B2 or B3, section not visible, surface red slipped on exterior with red rim band (10R4/3 weak red) interior on uncoated (7.5YR6/4 light brown), hand coiled and turned on turning device, base coiled and subsequently scraped, intact, a very clear and pure mud filling was still inside the pot, therefore it was not weighed.

\section{Nile B2 - Small squat jar with direct quadrifoil rim (Fig. 4.c)}

A small jar with quadrifoil rim has a wider rim band on the interior and the exterior. Made in a very similar way to the vessel above (8768) it is slightly squatter. Again the neck is relatively wide. Quadrifoil rims are common in the Tarif cemeteries A and B and Petrie's types 320 to 346 seem to cover this general type. ${ }^{51}$ These vessels were also

\footnotetext{
49 Petrie 1909, pl. XVII.

50 Seidlayer 1990, 82-83, 395.

51 Petrie 1909, pl. XVII, type 324 seems to fit exceptionally well.

52 SeILER 2012, 300-301, fig. 3-4.

53 Seidlmayer 1990, 82-83, 395.
}

put by Seiler in her Phase I.1 which covers the early $11^{\text {th }}$ Dynasty. ${ }^{52}$ Seidlmayer's seriation puts similar vessels (QA 244) in the $11^{\text {th }}$ Dynasty with most occurrences in his phases I and II. ${ }^{53}$

No. 8769 , rd. $=7.0-8.0$, $\max . \mathrm{d} .=10.0$, ht. $=10.3$, Nile B2, section uniform light brown as far as visible, surface dark red slip on exterior (10R5/6 red) and interior on rim (uncoated 10YR7/3 very pale brown), hand coiled and turned on turning device, base coiled and subsequently scraped, intact, weight $427 \mathrm{~g}$, in some places under quadrifoil rim the vessel was not well red slipped.

\section{Marl A4 - Small jar with direct everted rim (Fig. 4.d)}

This vessel type is distinguished from those before by the material - a marl clay fabric, and the presence of a narrower neck. The rim is direct and everted getting slightly thinner towards the orifice plane. The manufacturing technology is largely the same as described before but the vessel is more asymmetrical. Exact parallels are not shown in Seiler's work but Petrie's corpus suggests shape parallels from Tarif (e. g. his types $370,386^{54}$ ). Seidlmayer integrated this shape into his type QA 212, which appears in his Phases I to III equivalent to the $11^{\text {th }}$ and early $12^{\text {th }}$ Dynasty. ${ }^{55}$ No specifications on dating can be made as regards to the raw material.

No. $8770, \mathrm{rd} .=4.0$, max.d. $=8.8, \mathrm{ht} .=9.3$, Marl $\mathrm{A} 4$, section fine uniform olive greenish, surface uncoated ( $2.5 \mathrm{Y} 7 / 3$ pale yellow), hand coiled and turned on turning device, base coiled and subsequently scraped.

Finally the last in situ burial to be discussed here, 900-North 5 L9, is of a woman with a child ${ }^{56}$ with one vessel each made from Marl clay, both were found at the feet of the deceased (Fig. 2.d).

\section{Marl A4 - Small jar with direct everted rim (Fig. 4.e)}

The same chronological discussion as for no 8770 can be applied to the vessel described below. It has essentially the same form although the neck is slightly longer and the maximum diameter slightly

\footnotetext{
54 Petrie 1909, pl. XVII.

55 It seems as if type 386 might also have been grouped with QA 202: Seidlmayer 1990, 82-88, 395.

56 The anthropological study of the human bones was undertaken by Linda Chapon.
} 
less wide. An additional trait is the post-firing 'spout' broken into the rim, just as in one of the dishes above.

No. $8792, \mathrm{rd} .=4.0$, max.d. $=7.8, \mathrm{ht} .=9.0$, Marl A4, section uniform greenish, surface uncoated (2.5Y7/4 pale yellow), hand coiled and turned on turning device, base coiled and subsequently scraped on exterior, intact; weight $131 \mathrm{~g}$, found at the feet of the baby, a secondary, spout' has been broken into the rim in antiquity, perhaps to ease pouring out a liquid. The break is old. Stained with brown material on exterior.

\section{Marl A4 - Medium jar with straight neck and folded lip (Fig. 4.f)}

The medium jar made of the same fabric is not just a larger version of the vessel type discussed before but it shows again a wider neck and a folded lip. The proportions altogether are slightly different. For this type the cemeteries at Tarif provide at least a shape parallel (Petrie's type 401), which falls according to Seidlmayer exclusively into his Phase I, the early $11^{\text {th }}$ Dynasty. ${ }^{57}$

No. 8793, rd. $=7.0$, max.d. $=13.1$, ht. $=14.8$, Marl A4, section uniform yellowish/beige, surface uncoated (2.5Y7/4 pale yellow), hand coiled and turned on turning device, surface coiled and subsequently scraped, stained with brown material on exterior.

5.1.2.) Pottery types of the early Middle Kingdom found in the radîm around the tombs

The radim around these burials mentioned under 5.1.1. yielded more ceramic material, which in manufacturing technology, quality of fabric and surface treatment, as well as corpus fits well with the vessels found in situ. Among them a large number of sturdy mostly intact pointed beakers was found (cf. Fig. 3.d-1). In this section only vessels of that corpus that are not duplicated by the in situ vessels are described and discussed (mostly from surface context 910-North5-L3). ${ }^{58}$

\section{Nile B2 - Dish (Fig. 4.g)}

Simple vessel shapes such as this are notoriously difficult to date, but manufacturing technology, surface treatment and subtle details help to narrow

\footnotetext{
Seidlmayer 1990, 82-83, 395, QA240.

58 Bettina Bader would like to thank Daniela Penzer, Univ. of Munich, who drew most of these vessels.
}

down the range. In this case these indicators point towards the $11^{\text {th }}$ Dynasty none the least due to the incised horizontal groove on the interior and the quality of the fabric. Specific parallels cannot be cited, because Petrie's publication is not detailed enough to identify vessels like this with certainty. ${ }^{59}$

No. 8791, 910-N5-L5, rd.=16.6, max.d. $=17.3$, ht. $=5.1$, Nile B2, section grey core, red and brown oxidation zones, surface red slipped inside (2.5YR5/6 red) with red rim outside (7.5YR6/4 light brown), coiled and turned, base scraped in rough manner, smoke stained on interior ca. 50\% of the pot. A horizontal groove was incised ca $2.0 \mathrm{~cm}$ below the rim on the interior.

Nile B2/C1 - Small carinated dishes (Fig. 4.h-i) These small dishes or bowls seem to be derived from an older tradition with carinated contours. Although the carinations are not very pronounced their ancestry from 'Maidum'bowls in the widest sense seems clear. Their small size cannot be exactly paralleled in the Theban necropolis as yet, but similar shapes and materials were found at Tarif. ${ }^{60}$

No. 20137, 910-N5-L3, rd.=10.8, bd.= 3.2, ht.= 4.5 , Nile $\mathrm{B} 2 / \mathrm{C} 1$, section grey core, thin orange and light brown oxidation zones, surface red slipped inside and outside (2.5YR5/6 red), coiled and turned, base scraped and subsequently smoothed, tool cut.

No. 20153, 900-N5-L10, rd. $=12.8$, ht. $=4.5$, Nile B2, section not visible, pink slip inside and outside (7.5R7/4 pale red), nat. colour (10YR6/4-6 light yellowish brown to brownish yellow), coiled and turned, scraped, intact, $40 \mathrm{~g}$.

\section{Nile B2 - Small bowl (Fig. 4.j)}

The small bowl might belong to the $11^{\text {th }}$ Dynasty corpus of these burials because it is slightly deeper than the average small bowls and several similar types appear in Petrie's Tarif corpus (e.g. 107-108, $\left.112,118-120,129-131,133^{61}\right)$. Seidlmayer integrated them into different groups, namely QA144 and QA150 and the resulting seriation grouped those either across all his Phases (QA144) or in Phase II (QA150), which equals the entire $11^{\text {th }}$ Dynasty and

\footnotetext{
59 Perhaps Arnold 1968, 42 and Abb. 2.3, just shows such a vessel although the rim is more out-turned.

60 Arnold 1968, 47, Abb. 5.26-27.

61 Petrie 1909, pl. XIV.
} 
the $12^{\text {th }}$ up to Senwosret II or the second half of the $11^{\text {th }}$ Dynasty. ${ }^{62}$

No. $20138,910-N 5-L 3, \mathrm{rd} .=10.2$, bd. $=5.5$, ht. $=$ 4.8 , Nile B2, section uniform light brown, surface uncoated (7.5YR5/4 brown), coiled and turned or wheelmade (W1), base string cut.

\section{Nile B2/C1 - Small squat jar (Fig. 4.k)}

This small squat jar resembles those made of marl clay (nos 8792, 8770) although the body appears to be more squat than globular. This may be a direct result of the manufacturing technique, where the angular transitions of the joins of the vessel remained quite visible instead of being smoothed away. As for parallels, the same holds true for this vessel as those mentioned before. The dark red slip is worth noting because it is a very typical surface treatment for the earlier period at the site.

No. 20145, 910-N5-L3, rd.=6.2, $\max . \mathrm{d} .=9.4$, ht. $=10.2$, Nile B2/C1, section uniform brown, surface red slipped out and on rim in (2.5YR5/4 weak red), coiled and turned, base scraped, intact, $80 \mathrm{~g}$.

\section{Nile B2 fine - Small globular jar (Fig. 4.I)}

This vessel was unlike the other pottery entirely handmade, probably by opening and pinching a lump of clay. Only a few vaguely horizontal rilling lines were visible on the interior of the wide neck suggesting that no centrifugal force has been used at all. The fabric was exceptionally fine and micaceous. The base had also been trimmed and smoothed by hand on the exterior. Overall the vessel was very well smoothed although the wall thickness varied a lot across the pot. The short everted rim was direct but also not very well executed. Much of the vessel was smoke blackened or burnt probably due to its post-depositional history. In Petrie's corpus type $380^{63}$ is a viable parallel, but without the information about manufacturing technology, nothing further on dating can be said.

No. 20154, 900-N5-L9 ${ }^{64}$, rd.= 5.6, max.d.=9.1, ht.=9.2, Nile B2 fine, section uniform brown and black, surface uncoated (5YR4/3 reddish brown), smoke blackened (10YR3/1 very dark grey), handmade, base handmade.

\footnotetext{
Seidlmayer 1990, 81, 395.

63 Petrie 1909, pl. XVII.

${ }^{64}$ This jar was found very close to burial 900-North5-L9 but not in situ.
}

Nile B2/C1 - Medium bag shaped jar (Fig. 4.m) A slightly different variant to no. 8758 (Fig. 4.a) is shown here in order to demonstrate a possible range. Whilst this example is complete with flattened base, the technology described above is equally pertinent. Further differences can be seen in the height of the neck, and the length of the direct rim, which was trimmed. As for parallels the same suggestions can be made as before.

No. 20150, 910-N5-L3, rd.=6.5, max.d. $=16.7$, ht. $=20.0$, Nile B2/C1, section not visible, surface uncoated (2.5YR5-6/6 red), coiled and turned, base roughly scraped with a tool, slightly sooted on the exterior, only in lower third as if it stood in fire, complete, weight $2.8 \mathrm{~kg}$.

\section{Nile B2/C1 -Medium Hes - vases (Fig. 5.a-b)}

Two of the very widely distributed libation vessels were also found in the immediate vicinity of the early Middle Kingdom burials north of the temple enclosure. These two vessels differ in morphological details such as the form of the rim (straight everted or slightly turned down), the shape of the foot (tapering or with a slight 'foot') and the form of the shoulder (round or more pronounced). This form is widely distributed across the Theban necropolis and at Tarif a wide range of such vessels was found by Petrie. The type that seems most similar to no 20134 is Petrie's type 566, whilst type 579 resembles no 20133 although it does not have such a long neck. ${ }^{65}$ Almost no vessels in Petrie's corpus show pinched 'feet' or long necks or particularly round shoulders. Whether this is a question of detail in the old drawings or of dating will have to await further research. Interestingly the vessels shown by Arnold from Tarif and the Asasif both do not show these characteristics. ${ }^{66}$ Also none of the vessels published in the Middle Kingdom pottery Handbook fits exactly the description of those presented here. ${ }^{67}$

No. 20133, 910-N5-L3, rd.=6.6, max.d.= 12.2, bd.= 6.4, ht. $=22.5$, Nile B2, section red core, orange and light brown oxidation zones, surface red slipped outside with rim inside (10R4/6 red), coiled and turned (W1?), base cut with tool, complete, $2.4 \mathrm{~kg}$.

\footnotetext{
65 Petrie 1909, pl. XX. Both of these types went into Seidlmayer's type QA262, which in the seriation clustered in the Phases that he dated to the $11^{\text {th }}$ Dynasty. Seidlmayer $1990,91,395$.

66 Arnold 1972, Abb. 5.5.

67 Schiestl/Seiler 2012, 508-510.
} 

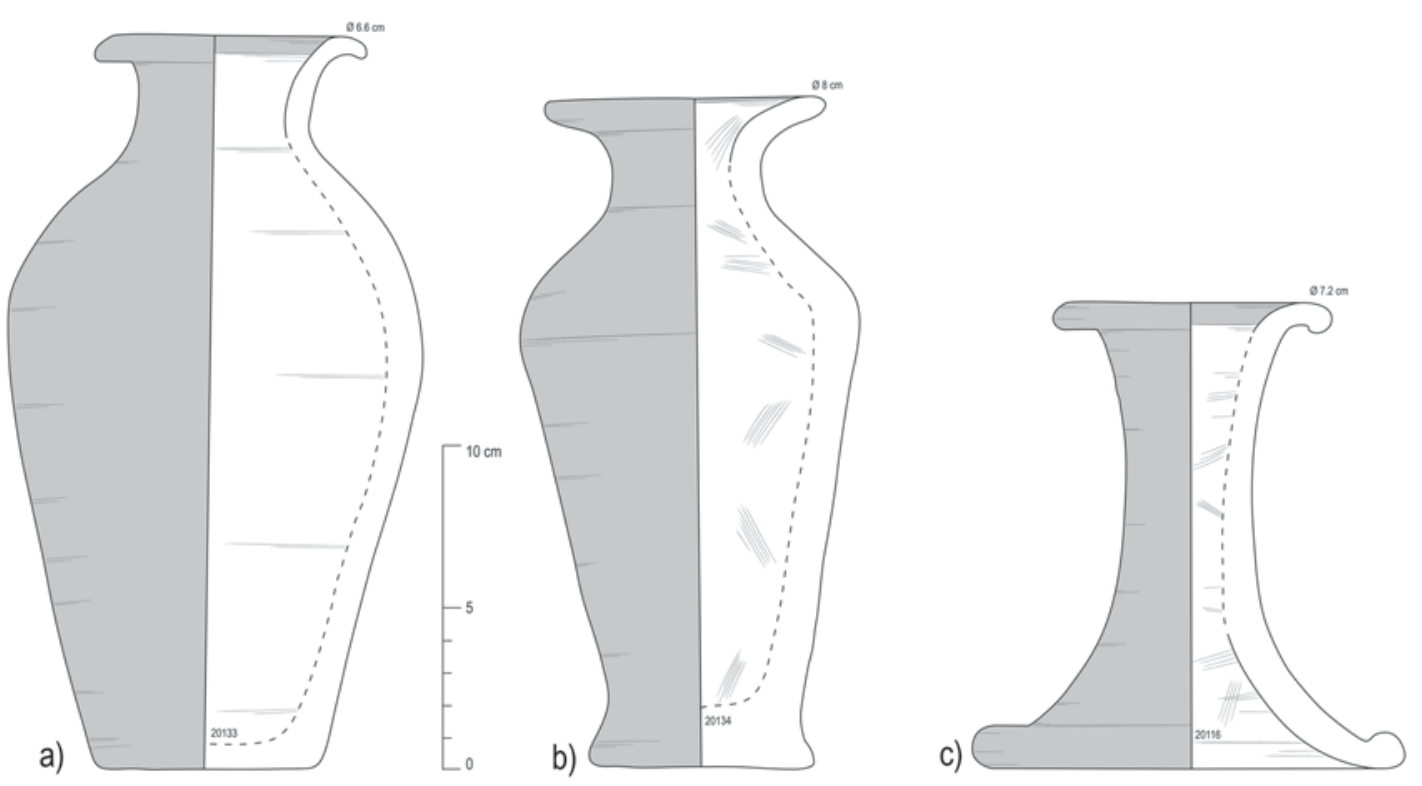

Fig. 5 Pottery from radîm 900-N5-L3, 11 $1^{\text {th }}$ Dynasty, drawn by D. Penzer, inked by E. Schuster.

No. 20134, 910-N5-L3, rd.= 8.0, max.d. $=10.4$, bd. $=6.6$, ht. $=20.6$, Nile $\mathrm{B} 2 / \mathrm{C} 1$, section uniform brown, surface red slipped outside and on rim inside (10R4/6 red), coiled and turned, base cut with tool and smoothed.

\section{Nile B2/C1 - Medium tall stand (Fig. 5.c)}

Of note is also the stand found in the vicinity of the early Middle Kingdom burials. The thick dark red slip of the surface and the good quality of the execution are remarkable. There are no direct parallels from Tarif for this stand but generally taller stands tend to be earlier in time than the ubiquitous lower ones of the later Middle Kingdom. The Middle Kingdom pottery Handbook lists this type for the whole duration of the Middle Kingdom although there are no examples from the Theban necropolis. ${ }^{68}$

No. $20116,910-\mathrm{N} 5-\mathrm{L} 3, \mathrm{rd} .=7.2$, bd. $=12.0$, ht. $=$ 14.3, Nile $\mathrm{B} 2 / \mathrm{C} 1$, section red core, orange and brown oxidation zones, surface red slipped outside and on rim inside down to a quarter of the height (7.5R5/6 red), coiled and turned (W1?), base scraped on interior and well smoothed exterior.

\subsection{3.) Summary}

These burials are well in accord with some burial assemblages of Petrie's cemeteries A and B at Tarif, which he dated to the ' $11^{\text {th }}$ Dynasty'. ${ }^{69}$ Seidlmayer's analytic work on this material, which still provides the largest body of comparanda available to date, enabled us to see the development that took place and to get closer to a date for the group of graves north of the temple of Thutmosis III, namely to the first two phases that he defined (encompassing the $11^{\text {th }}$ Dynasty). First of all it is important to learn that very simple graves of a 'poorer stratum' of people belonging to this time horizon are to be found a considerable distance to the south of Tarif. Whether the tombs rather belong to the time horizon of the Intef kings buried in the saff tombs of el-Tarif or to that of Mentuhotep II buried in Deir el-Bahari cannot yet be answered with certainty. Arnold's study of the material from Tarif and the Asasif, however, suggests a closer relationship of the Thutmosis III tomb cluster to Tarif than to Deir el-Bahari and the Asasif, ${ }^{70}$ although it was not in situ and although we know now that some of it must be later

\footnotetext{
70 Arnold 1972, Abb. 2-5. It is clear though that some of the material from the Asasif, such as the large jar no. 9 in Abb. 4 and the globular jars with everted rims no 11 belong to a later phase of the Middle Kingdom.
} 
than the early Middle Kingdom. Unfortunately, much ceramic material from the relevant periods from the Asasif remains unpublished. Published evidence from Dra Abu el-Naga does not seem to have an immediate bearing on the dating of the material presented here.

\section{2.) The pottery from the Middle Kingdom tombs within the temple area}

At the time of writing twelve rock cut corridor and shaft tombs have been brought to light in the area of the mortuary temple of Thutmosis III. All of them were disturbed and/or destroyed by the construction of the temple. The tombs often contained mixed ceramic material of the early Middle Kingdom, the late Middle Kingdom, the Second Intermediate Period and the early New Kingdom. A few deposits can be isolated, which may constitute the original burial assemblages although they were probably shifted from their original positions, either by intruders to rob the tombs or by parties preparing the next burial (e.g. tomb III). Nevertheless the ceramic material is presented here tomb by tomb and deposit by deposit in order to make the material available to the scientific community in its find contexts. In several cases it is possible to discern a sequence of use phases of these tombs, which were part of a larger Middle Kingdom cemetery in this area in Thebes encompassing the northern part of the Ramesseum and the mortuary temples of Amenhotep II, Thutmosis III and Thutmosis IV.71

In this instance the discussion of comparanda will also concentrate on the Theban area, except in cases where only external parallels are known.

\subsection{1.) Tomb II (in squares 990 -South3/South4)}

Tomb II was excavated in 2009 and is situated in the south western part of the temple close to the stone pavement still preserved and recently restored. Whilst fragments of four sarcophagi were noted during the excavation, ${ }^{72}$ only parts of two polychrome coffins dating generally to the Middle Kingdom/Second Intermediate Period could be restored. ${ }^{73}$

\footnotetext{
71 Nelson/Kalos 2000; Nelson 2003; Nelson 2013; Sesana/ Consonni 2013; Quibell 1898, 3-5; Guidotti/Silvano 2003, 20-21; GuidotTI 1985.

72 Seco Álvarez et al. 2012/13 (appeared 2016), 337.

73 Seco Álvarez et al. 2012/13 (appeared 2016), 357.
}

The ceramic material from this tomb comprised a small and very broken assemblage from 900-South3-4 L4 (Fig. 6). This group of pottery can be called typical for the late Middle Kingdom and perhaps the early Second Intermediate Period, without any obvious later intrusions. The body sherds of this context are in keeping with such a proposition (fragments of Nile B2 open shapes; Nile B2 closed shapes; Nile C 'beer jars' and bread moulds). This does not mean that the context was necessarily in situ. This impression may be strengthened by the fact that all vessels are only preserved as fragments.

\section{Nile B1 - Hemispherical cups (Fig. 6.a-b)}

The rims of hemispherical cups are of widely open shape with a diameter of around 13.0 to $14.0 \mathrm{~cm}$ and a red rim band. These vessels are a type fossil in the Middle Kingdom and have a huge list of comparanda all over Egypt. ${ }^{74}$ Because dating by external parallels is seen in a very critical light and leads to circular arguments, ${ }^{75}$ mainly parallels from the Theban necropolis and the Theban east bank are mentioned here such as Tomb ${ }^{\prime} 79^{76}$ and the earlier shafts of the ' $13^{\text {th }}$ to $16^{\text {th }}$ Dynasty' at Dra Abu el-Naga ${ }^{77}$.

Tomb II-L4-2, rd.= 13.0, pres. ht.=4.5, Nile B1, surface uncoated with thin red slipped rim band on interior and exterior, coiled and turned (W1).

Tomb II-L4-3, rd.= 14.0, pres. ht.= 2.8, Nile B1, surface uncoated with thin red slipped rim band on interior and exterior, coiled and turned (W1).

\section{Nile B2 - Small shallow dish with bulbous lip} (Fig. 6.c)

This singular small fragment has so far no parallels in the Theban necropolis, although the ware and technology hints at a date in the Middle Kingdom.

Tomb II-L4-4, rd.= 11.0, pres. ht.=2.0, Nile B2, surface eroded on interior, dark red slip on exterior, probably coiled and turned.

\section{Nile B2/C1 - Small dishes with everted rims (Fig. 6.d-e)}

The two dishes subsumed under this heading are slightly different in contour, the first with a slight

\footnotetext{
Schiestl/Seiler 2012, 84-110, esp. 96-97.

BAder 2007; Bader 2009, 149-159.

76 Guidotti 1985, 53, n. 11-n17.

77 Seiler 2005, 53, Abb. 12.
} 
a)
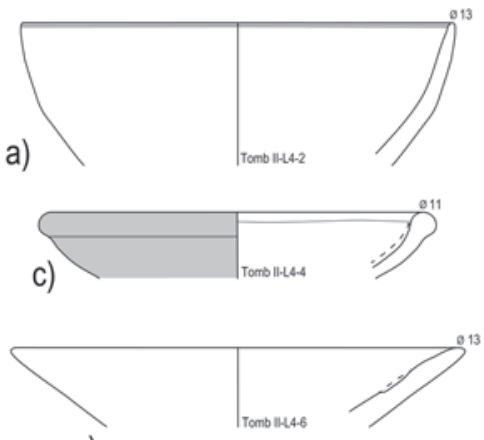

e)

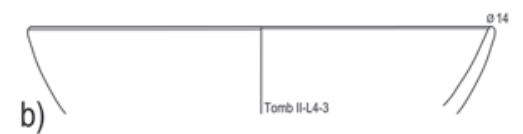

d)
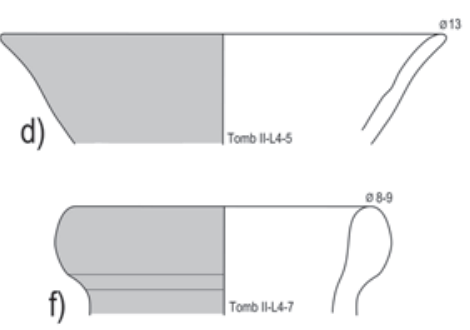

h)
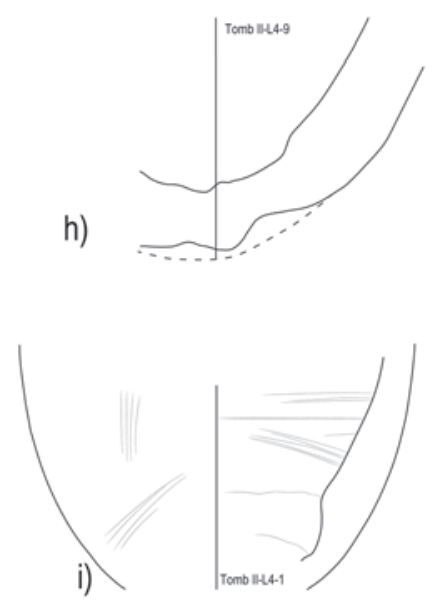

g)

Fig. 6 Pottery from Tomb II late Middle Kingdom/early Second Intermediate Period, drawn by B. Bader, inked by E. Schuster.

s-curved contour, whilst the other is straight. Both of them have direct rims and are eroded. Their fragmentary state only allows a rough comparison perhaps with carinated or conical cups. ${ }^{78}$

Tomb II-L4-5, rd.= 13.0, pres. ht. $=3.4$, Nile B2/ $\mathrm{C} 1$, surface eroded interior, dark red slip on exterior, probably coiled and turned.

Tomb II-L4-6, rd.= 13.0, pres. ht.= 2.5, Nile B2/ $\mathrm{C} 1$, surface uncoated, probably coiled and turned.

\section{Nile C - 'Beer' jars (Fig. f-h)}

The diagnostic fragments of beer jars found in this context do not only suggest kettle shaped rims and ovoid body shapes, but also that the fabric variant with charred plant remains seen in some types in the New Kingdom also existed already in the late Middle Kingdom (see Fig. 1.f). The kettle rim can be paralleled at Dra Abu el-Naga in the late Mid-

78 Schiestl/Seiler 2012, conical cups: 120-121, carinated cups: $292-297$. dle Kingdom/early Second Intermediate Period phase, ${ }^{79}$ as well as in the Ramesseum. ${ }^{80}$

Tomb II-L4-7, rd.=8.0-9.0, pres. ht.= 3.3, Nile $\mathrm{C} 4$, surface red slipped on exterior, interior eroded, coiled and turned (W1). The section of the kettle rim is shown in Fig. 1.f, the wall thickness is $1.3 \mathrm{~cm}$.

Tomb II-L4-8, pres. ht.= 18.8, Nile C2 + some lime, surface uncoated? eroded, coiled and turned, based roughly scraped on exterior, asymmetric.

Tomb II-L4-9, pres. ht.= 7.4, Nile C4, surface totally eroded, probably coiled and turned, base scraped on exterior.

Marl A4 - Small or medium closed vessel (Fig. 6.i)

The body fragment of a small or medium vessel cannot be compared to other material. The soft-

\footnotetext{
SEILER 2005, 74-75, DAN 3.

80 Loyrette et al. 1993, fig. 4.m-n.
} 
ness of the vessel wall, the technology and the fabric seem to indicate a general Middle Kingdom date for this fragment.

Tomb II-L4-1, pres. ht.= 7.6, Marl A4, for section see Fig. 1.i, surface uncoated, coiled and turned, base trimmed and smoothed.

\subsection{2.) Tomb III (squares 930-940-950-South2-3)}

Tomb III was also located in the south western part of the temple and encompassed a sloping corridor with east-west orientation and a niche cut into the soft bedrock as well as a vertical shaft and one burial chamber. ${ }^{81}$ A preview of the ceramic material of this tomb has already been published. ${ }^{82}$

Progress has been made on the analysis of the material from Tomb III. It was possible to reconstruct twelve full profiles of Holthoer's BB jars with direct rim as well as five more base/body fragments from context 940-South2-L11. They all seem to belong to Holthoer's type BB2 transition$\mathrm{al},{ }^{83}$ but final recording needs to confirm this. It is interesting that so many vessels of the same recognisable type came from one deposit. But other New Kingdom types were also found in this context, such as no. 8370 (cream slipped sherd of a closed vessel with red and black lines), no. 8369 (Marl A4 closed vessel) as well as a Middle Kingdom carinated bowl (no. 9190).

Further study is necessary to ascertain whether this deposit is derived from activities around the tomb or the temple itself. Interestingly several of the BB jars were made of uncoated Nile B4 with a very soft feel to the surface. In another context around the tomb a number of hemispherical cups and other Middle Kingdom vessels were found. They are largely congruent with what is presented below. A very close parallel to Tomb III itself and the material found within it is represented by Tomb ' 79 in the area of the mortuary temple of Thutmosis IV. ${ }^{84}$ This includes the two offering trays/soul houses derived from L17, which are not republished here. ${ }^{85}$

\section{Nile B2 - so-called Beer bottle after Holthoer (BB jar, Fig. 7.a-b)}

The examples of this type are quite well made if compared with others. Bader's base type is D with cutting round the edges, but the vessel can stand up nicely. For a more in depth discussion on this type see below $(7.2 ; 89)$.
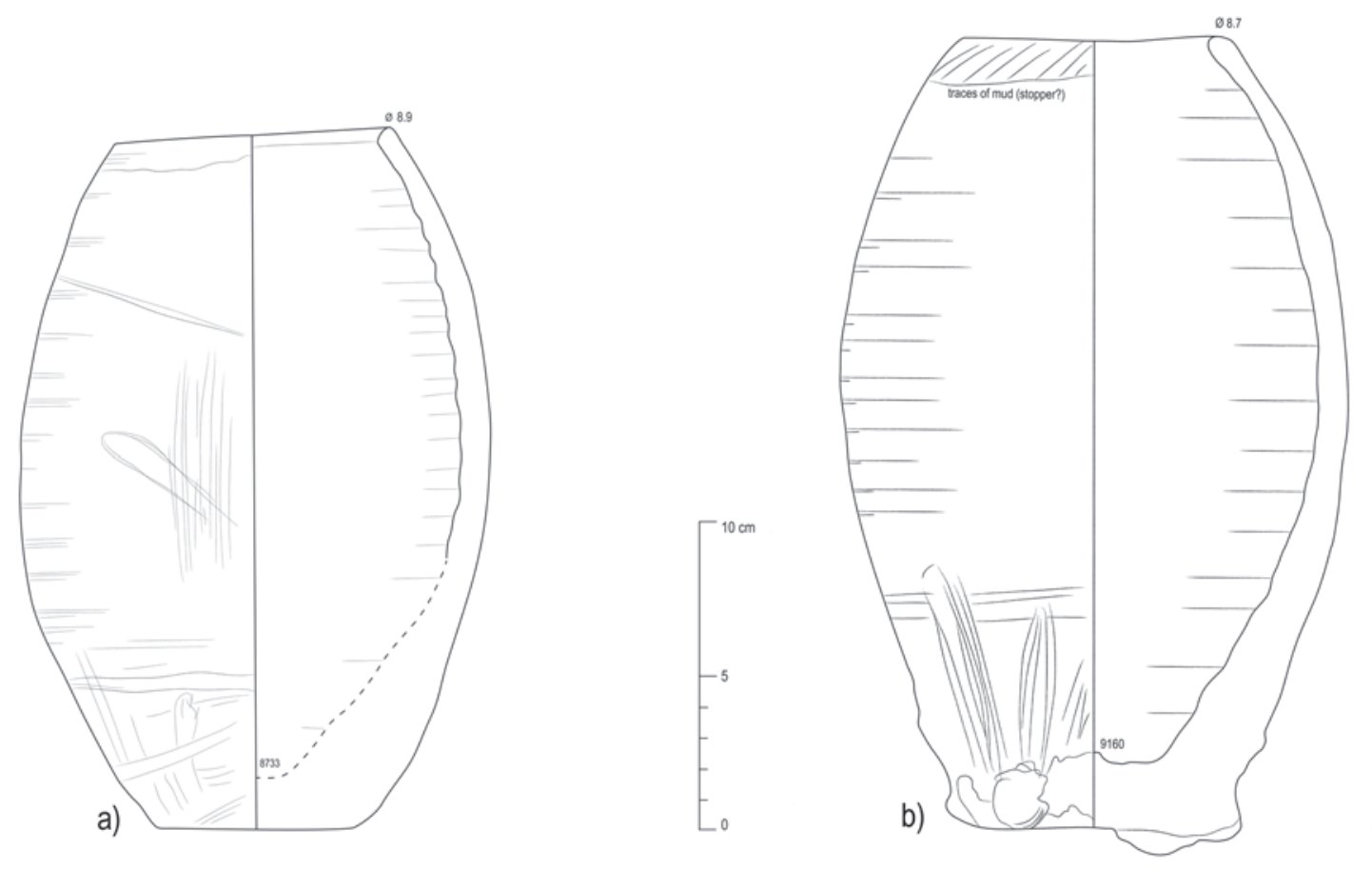

Fig. 7 BB jars from Tomb III-L11, New Kingdom, drawn by B. Bader, inked by E. Schuster.

81 Archaeological description and plan, Seco ÁlvVarez et al. 2012/13 (appeared 2016), plans 5 and 7.

82 Seco Álvarez et al. 2012/13 (appeared 2016), 359-364, fig. 36.3-h, 37.a-c.
83 HOlthoer 1977, pl. 18.

84 Guidotti 1985.

85 Bader in Seco Álvarez et al. 2012/13 (appeared 2016), 360-362. 
No. 8733 , rd. $=8.9$, max.d. $=15.1$, bd. $=6.2$, ht. $=$ 22.4, Nile B2, section not visible, surface uncoated (5YR5/4 reddish brown), wheelmade (W1), base string cut, complete, $1.6 \mathrm{~kg}$.

No. 9160 , rd. $=8.7$, bd. $=9.0-9.3$, max.d. $=16.4$, ht. $=24.2-26.1$, Nile B2/C1, section uniform reddish brown, surface uncoated (5YR6/4 light reddish brown), wheelmade, base string cut and hand modelled, traces of mud filling and mud stopper around the rim.

The pottery from the 'patio' 930-South2$\mathbf{L 1 0}^{86}$ contains several types of pottery vessels all of which belong to the Middle Kingdom. The question remains whether they are derived from single or repeated use of this tomb. The good state of preservation suggests that the deposit was pushed aside by later users of the tomb.

\section{Nile B1 - Hemispherical cups (Fig. 8.a-d)}

Four hemispherical cups with complete profile offer a range of vessel indices from 205 to 225 . Only one of them has a thin red rim band (Fig. 8.b), the others remain uncoated. The quality of these cups is very good and the bases are all trimmed with a tool creating a pattern. The rim diameter is rather wide from 12.3 to $13.0 \mathrm{~cm}$. The best parallels are from Tomb '79 with very similar dimensions and surface treatment. ${ }^{87}$ Those from Dra Abu el-Naga dating to the late Middle Kingdom seem to be different. ${ }^{8} 8$

No. 9169, rd. $=12.6-12.8$, ax.d. $=12.8$, ht. $=5.7$, Nile B1, section not visible, surface uncoated (7.5YR6/4 light brown), asymmetric, coiled and turned (W1), base trimmed with a tool, complete, vessel index 224.6, base scraped in Memphis style. ${ }^{89}$

No. $9176, \mathrm{rd} .=13.0$, ht. $=6.0$, Nile B1, section uniform brown, surface uncoated (7.5YR6/4 light brown) with thin red rim band (10 $\mathrm{R} \mathrm{5/6}$ red), coiled and turned (W1), base trimmed with a tool, vessel index 216.7.

No. 9177, rd. $=12.3$, ht. $=6.0$, Nile B1, section uniform brown, surface uncoated (10YR7/3 very pale brown), coiled and turned (W1), base trimmed with a tool, vessel index 205.

No. $9178, \mathrm{rd} .=12.6, \mathrm{ht} .=5.6$, Nile B1, section uniform brown, surface uncoated (7.5YR6/3 light

\footnotetext{
86 A selection of pottery from this context was presented by Bader in Seco Álvarez et al. 2012/13 (appeared 2016), 359-360.

87 Guidotti 1985, 53, n. 11-17.

88 SeILer 2005, Abb. 12.
}

brown), coiled and turned (W1) base trimmed with a tool, vessel index 225 .

\section{Nile B1 - Small bowl (Fig. 8.e)}

Comparanda and dating criteria for small dishes and bowls are difficult to find and the dates usually cover a long period..$^{90}$

No. 9175, rd. $=10.0$, bd. $=3.7$, ht. $=3.6-4.3$, Nile B1, section uniform brown, surface uncoated (7.5YR7/3 pink), coiled and turned, base string cut.

\section{Nile C2 - Carinated bowl with undulating rim and ring base (Fig. 8.f)}

This large bowl, one of three found altogether in the tomb (see Fig. 10.a, 12.g), does not appear in this particular form in the Middle Kingdom Handbook, although due to peculiarities of fabric and technology there can be no doubt that it belongs to the Middle Kingdom. The extraordinary good quality even points to an early date in the Middle Kingdom for this type.

No. 9155, rd. $=30.3, \mathrm{bd} .=8.3$, ht. $=15.3$, Nile C2, section dark grey core with brown oxidation zones, surface red slipped on interior and on exterior down to the carination, splashed below (10R5/6 red), natural surface 5YR5/4 reddish brown, coiled and turned, interior well smoothed, lower part trimmed on exterior and separate pad added to form ring base; before trimmed and smoothed.

Nile B2/C1 - Beaker with pointed base (Fig. 8.g) In contrast to the beakers of the early Middle Kingdom (Fig. 3.d-1), this type represents a later variant with a wider and longer top part. This type appears in Thebes in the late $11^{\text {th }}$ and early $12^{\text {th }}$ Dynasty. ${ }^{91}$ The parallels given in the Handbook suggest that this pottery type is a Theban or at least southern invention.

No. 9172, rd.=7.5-7.8, ht. $=15.0$, Nile B2/C1, section not visible, surface uncoated (7.5YR6/4 light brown), breaking and pinching, top of vessel turned, base handmade/pinched, join visible.

\section{Nile C2 - Base of large closed vessel ('beer' jar? Fig. 8.h)}

Large truly globular bodies are basically only paralleled by Middle Kingdom 'beer' jars, which

\footnotetext{
89 This represents a very particular scraping pattern of the base going across the base and around it.

90 Schiestl/Seiler 2012, Hyperboloid model bowls, 856-858.

91 Schiestl/Seiler 2012, 132-135, esp. nos 6-7 from the early $12^{\text {th }}$ Dynasty.
} 

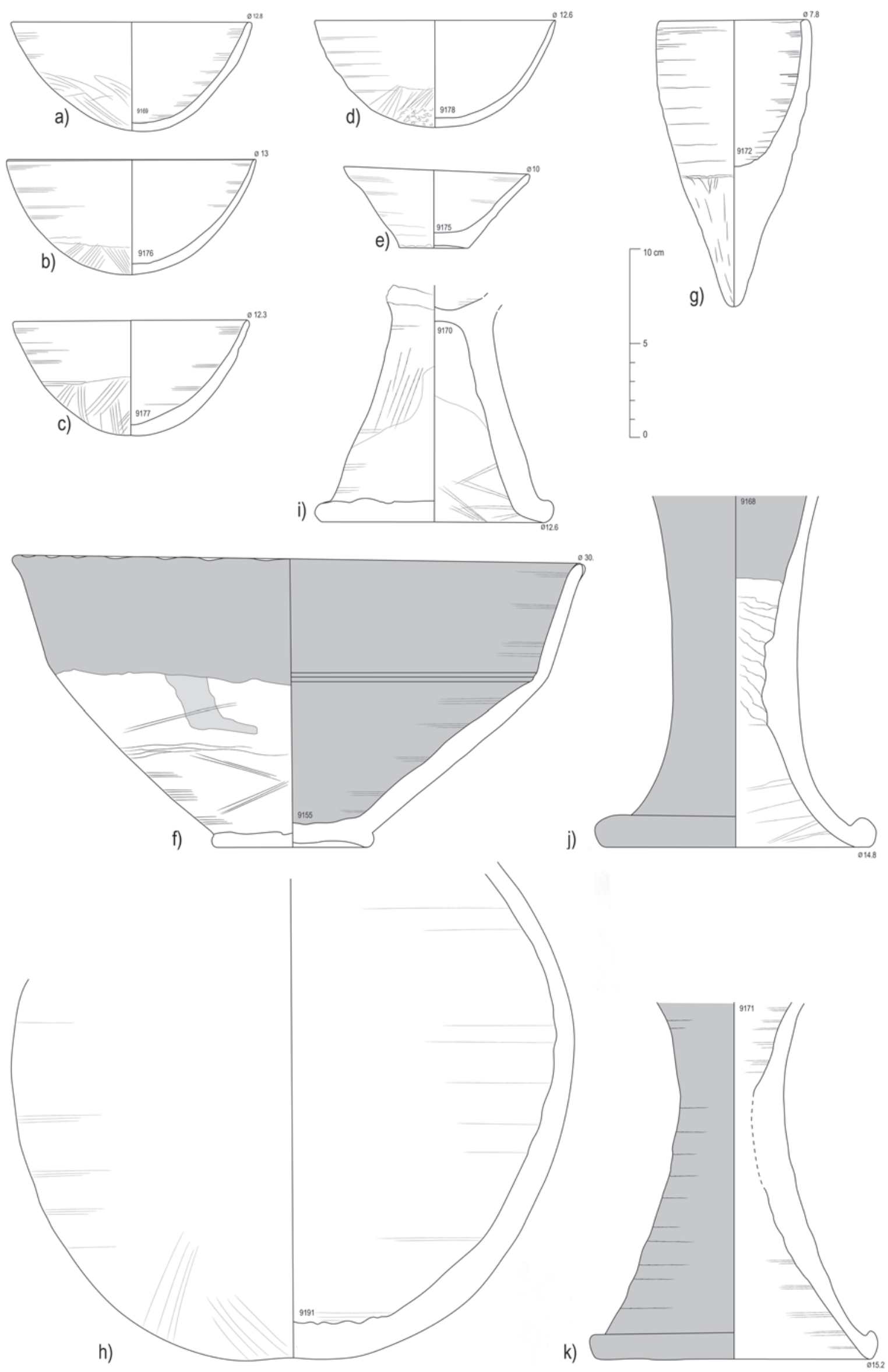

Fig. 8 Pottery from Tomb III-L10, early Middle Kingdom, drawn by B. Bader, inked by B. Bader and E. Schuster. 
were only found in the Theban necropolis early in the $12^{\text {th }}$ Dynasty. ${ }^{92}$ This fits well with the contents of Tomb '79 beneath the temple of Thutmosis IV, where also only bodies without the necks were preserved..$^{93}$

No. 9191, pres. ht. $=26.3$, Nile C2, section uniform light brown, surface probably uncoated, badly eroded (10YR6/4 light yellowish brown), coiled and turned, base scraped with a tool. The section is shown in Fig. 1.d, thickness of the wall ca $0.9 \mathrm{~cm}$.

\section{Nile B2 - Offering stand with dish (Fig. 8.i)}

Only the feet of this offering stand were preserved.

No. 9170 , bd. $=12.6$, pres. ht. $=12.6$, Nile B2 + some limestone, section olive core, red oxidation zones, surface uncoated (5YR5/6 yellowish red), coiled and turned, base hand formed and trimmed, surface obscured by blackening, smoke blackening/burning outside and inside foot.

\section{Tall stands various fabrics (Fig. 8.j, k)}

These stands are also paralleled well in Tomb '79 beneath the temple of Thutmosis IV. ${ }^{94}$

No. 9168, max.d. $=14.8$, bd. $=14.0$, pres.ht. $=$ 19.1, Nile B2 + limestone, section uniform light brown, surface dark red slipped and polished on exterior and interior inside down to mid height, very good quality, coiled and turned, the middle of the stand is squeezed together and turned like a beer bottle neck of the MK, base scraped on the inside but well smoothed only base preserved.

No. 9171, max.d. $=15.2$, bd. $=14.3$, pres.ht. $=$ 19.2, Nile C2, section uniform light brown, surface red slipped and polished on exterior (10R6/6 light red), natural surface colour (10YR6/6 brownish yellow), coiled and turned, the middle of the stand is hand formed, base was wheel turned, it remains unclear if the preserved end is really the base, only splashes of red slip on interior.

The Corridor Entrance Deposit 940-South2$\mathbf{L 1} 7^{95}$ consisted of a larger number of complete and largely reconstructable vessels of high quality in

\footnotetext{
Schiestl/Seiler 2012, 406-407.

Guidotti 1985, 60, n. 79-80.

GuidotTi 1985, 58, n. 71, 59, n. 73.

95 Seco Álvarez et al. 2012/13 (appeared 2016), see fig. 12 for the context.
}

combination with two offering tables/soul houses also produced from pottery one of which was rectangular, whilst the other was oval. ${ }^{96}$

\section{Nile B1 - Hemispherical cups (Fig. 9.a-c)}

The vessel indices of the cups with complete profiles are around 200, thus a little smaller than in L10. It is unlikely, though, that this has any chronological significance because the raw material is very similar to the material above and some vessel types such as the cups, the carinated bowls, the pointed beakers and the globular jars all re-appear in this context. The rim diameters are very similar.

No. 9203, rd. $=12.2$, ht. $=6.1$, Nile B1, section uniform brown, surface uncoated (5YR5-6/4 light reddish brown to reddish brown), coiled and turned (W1), base scraped with a tool, vessel index 200.

No. $8721, \mathrm{rd} .=13.8, \max . \mathrm{D}=13.8, \mathrm{ht} .=6.7$, Nile B1, section uniform light brown, surface uncoated (7.5YR6/4 light brown) with red rim band (10R5/8), coiled and turned (W1), base trimmed on the outside, vessel index 205.97.

940-S2-L17-1, rd. $=13.0$, pres.ht. $=5.9$, Nile B1 + lime, section uniform light brown, surface uncoated (7.5YR6/6 reddish yellow) with thin red rim band (10R5/8 red), coiled and turned, very well smoothed, base trimmed.

\section{Nile B1 - Shallow dish/plate with white dots on interior (Fig. 9.d)}

This dish was produced to a very high quality and similar fragments with white dots have been found in Tomb ' 79 beneath the temple of Thutmosis IV. ${ }^{97}$ Whilst dishes with such decoration were found in the later Second Intermediate Period phase at Dra Abu el-Naga, ${ }^{98}$ the shape of the vessel is more in keeping with undecorated ones of the early Middle Kingdom. ${ }^{99}$

No. 9161, rd. $=18.7-19.3$, ht. $=6.3$, NileB1/B2, section not visible, surface red slipped inside (10R5/6 red), natural surface colour (7.5YR5/4 brown), very well smoothed, coiled and turned, base trimmed/scraped, complete.

\footnotetext{
Published with a discussion and parallels together with a small selection of pottery from L17 by BADER, in SECO Álvarez et al. 2012/13 (appeared 2016), 360-362, fig. $35-36$.

97 Guidotti 1985, 61, n. 93

98 SeILER 2005, 80-81, Abb. 34.

99 Guidotti 1985, 52, n. 8; Schiestl/Seiler 2012, 196, shallow inflected bowls.
} 

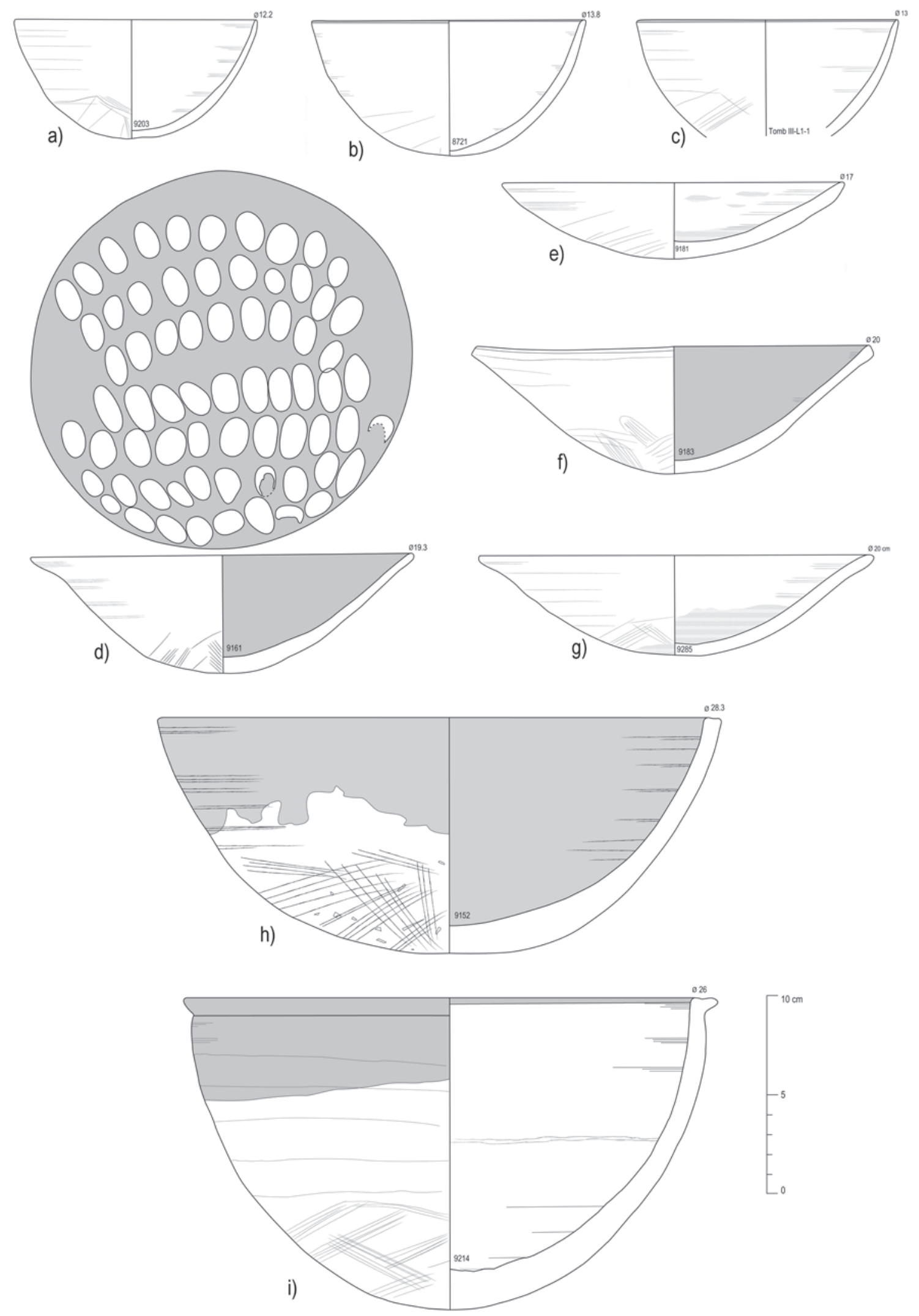

Fig. 9 Pottery from Tomb III-L17, early Middle Kingdom, drawn by B. Bader, inked by B. Bader and E. Schuster. 
Nile B2 - Shallow dish/plate (Fig. 9.e)

Very few parallels exist for such very shallow dishes of high quality. One may be seen in the temple of Thutmosis IV although it is smaller. ${ }^{100}$

No. 9181, rd.= 17.0-17.2, ht.= 3.8, Nile B2 + limestone, section not visible, surface uncoated (7.5YR6/4 light brown), coiled and turned, base scraped with a tool, intact.

\section{Nile B2 - Dishes with round bases (Fig. 9.f-g)}

Whilst dishes with inflected contour occur in the earlier part of the Middle Kingdom and also in the temple of Thutmosis IV, ${ }^{101}$ the dish with trimmed and red slipped rim lacks a direct parallel although the shape and technology are in keeping with the other material.

No. 9183, rd. $=20.0$, ht. $=6.4$, Nile B2, section not visible, surface red slipped inside (7.5R6/6 light red), uncoated on exterior (5YR6/6 reddish yellow), coiled and turned, very well smoothed, base scraped with a tool, complete, rim trimmed with tool on top.

No. 9285 , rd. $=20.0$, ht. $=5.1$, Nile B2 + limestone, section uniform light brown, surface uncoated (7.5YR7/4-6 pink to reddish yellow), coiled and turned, base scraped with a tool, interior smoke blackened.

\section{Nile C2 - Large bowls and basins (Fig. 9.h-i)}

Such bowls made of Nile $\mathrm{C}$ with a variety of surface treatments show longevity as they seem to occur throughout the whole duration of the Middle Kingdom except in the $11^{\text {th }}$ Dynasty. They may have a trimmed top of the rim or show a small folded lip that was also trimmed. ${ }^{102}$

No. 9152, rd. $=28.3$, ht. $=11.8$, Nile $\mathrm{C} 2$, section not visible, surface red slipped on interior, on exterior irregular down to mid body (10R5/6 red, natural surface 10YR6/3 pale brown), coiled and turned, base scraped with a tool, intact.

No. 9214, rd. $=26.0$, ht. $=15.5$, Nile $\mathrm{C} 2+$ limestone, section dark grey core with red and brown oxidation zones, surface with wide red rim band outside, thin red rim band inside (10R4/6 red, natural surface 5YR4/4 reddish brown), well smoothed inside, outside rough, coiled and turned, base scraped, top of rim trimmed with tool.

\footnotetext{
100 GuidotTi 1985, 52, n. 7.

101 Schiestl/Seiler 2012, 196, especially no 21 from temple of Thutmosis IV.
}

Nile C2 - Large carinated bowl with undulating rim (Fig. 10.a)

This type was already discussed above (cf. Fig. 8.f).

No. $9154, \mathrm{rd} .=26.0, \mathrm{bd} .=10.3$, ht. $=14.6-14.9$, Nile C2, section not visible, surface red slipped inside and outside below carination (7.5R6/6 red, natural surface 7.5YR6/4 light brown), coiled and turned, base scraped and pad with ring subsequently added, ring handmade and added separately, complete.

\section{Nile B2 - Conical beaker with pointed base} (Fig. 10.b)

This type was already discussed above (cf. Fig. 8.g).

No. 9193, rd.=9.0-10.0, ht. $=15.5$, Nile B2 + limestone, section uniform light brown, surface uncoated (7.5YR7/4 pink), combination technique wheelmade and handmade, base hand formed.

\section{Nile C2 - 'Beer' jar (Fig. 10.c)}

This example of a large jar with a narrow cylindrical neck shows a heavily folded rim and a complete red slip on the exterior. Such vessels seem to be particularly frequent in the northern part of Egypt in the early 12th Dynasty. ${ }^{103}$

No. 9158, rd. $=11.2$, max.d. $=27.6$, ht. $=36.7$, Nile C2 + limestone, section not visible, surface red slip outside (2.5YR6/8 red, natural surface 2.5YR6/8), well smoothed, coiled and turned, base scraped on the outside, large crack in rim, but otherwise intact.

\section{Nile B1, Nile B2 and Nile C2 - Model dishes} and bowls (Fig. 10.d-s, Fig. 11.a-g)

The small dishes, usually carelessly 'wheelmade' with string cut bases and rather carelessly manufactured are collected here rather by shape than by fabric. Some vessels have a white rim band. The variation ranges from very fine Nile $\mathrm{B} 1$ to the rough end of Nile B2 with a sizable group of Nile $\mathrm{C}$ dishes. Whether they were just small versions of ordinary bowls or by their function defined as 'models' must remain unclear from the current find context. What can be said is that in the overall Middle Kingdom corpus there are no exactly

\footnotetext{
102 Schiestl/Seiler 2012, 314-320.

103 Schiestl/Seiler 2012, 412-413.
} 
equivalent vessels that happen to be just larger with the same morphological details. For this reason it may not be justifiable to name these vessels models. A good range of parallels is found in Tomb ' 79 beneath the temple of Thutmosis IV..$^{104}$

No. 9209, rd. $=11.1-11.3$, bd. $=4.5-4.8$, ht. $=$ 3.6-4.0, Nile B1, section not visible, surface uncoated (5YR5-6/6 yellowish red to reddish yellow) with white rim inside (5Y8/1 white), wheelmade, base string cut, complete.

No. 9208, rd. $=9.4-9.7$, bd. $=3.5$, ht. $=3.6$, Nile B2, section uniform brown, surface uncoated (7.5YR6/3 light brown), wheelmade, base string cut

No. 9166 , rd. $=10.1$, bd. $=4.0$, ht. $=2.9-3.3$, Nile B2, section uniform brown, surface uncoated (7.5YR6/3 light brown) with white rim band, wet smoothed, wheelmade, base string cut, almost complete.

No. 9212, rd. $=10.7, \mathrm{bd} .=4.0$, ht. $=2.6$, Nile B2, section uniform brown, surface uncoated (7.5YR6/4 light brown) with white rim inside (2.5YR8/1 white), wheelmade, base string cut.

No. 9213 , rd. $=10.8, \mathrm{bd} .=4.6$, ht. $=3.1-3.6$, Nile B2, section red core brown oxidation zones, surface uncoated (5YR6/6 reddish yellow) with white rim inside (10YR8/1 white), wheelmade, base string cut.

No. 9192, rd.= 10.0, bd.=3.7-4.0, ht.=3.1-3.7, Nile B2 + limestone, section not visible, surface uncoated (7.5YR6/4 light brown), wet smoothed, wheelmade, base string cut, complete.

No. 9201, rd.=7.2, bd. $=3.7-4.0, \mathrm{ht} .=2.0-2.3$, Nile B2 + limestone, section uniform dark brown, surface uncoated (7.5YR6/4 light brown), careless manufacture, wheelmade, base string cut.

No. $9202, \mathrm{rd} .=8.5-9.2$, bd. $=3.5-4.5$, ht. $=2.3-$ 2.8 , Nile B2 + limestone, section not visible, surface uncoated (7.5YR6/4 light brown) wheelmade, base string cut, intact.

No. 9206, rd. $=10.2$, bd. $=3.8$,ht. $=2.9-3.1$, Nile B2 + limestone, section uniform brown, surface uncoated (7.5YR6/4 light brown), wheelmade, base string cut, almost complete, white colour on the outside on one side of the pot only -accidental??

No. 9198, rd. $=7.8-8.2$, bd. $=4.5-4.8$, ht. $=1.9-$ 2.1, Nile $\mathrm{B} 2 / \mathrm{C} 1$ + limestone, section not visible, surface uncoated (7.5YR6/4 light brown), wheelmade, base string cut, intact.
No. 9330, rd.=9.2-9.5, bd. $=3.3-4.0$, ht. $=3.1-$ 3.5, Nile $\mathrm{B} 2 / \mathrm{C} 1$ + limestone, section uniform brown, surface uncoated (7.5YR6/4 light brown), wet smoothed, wheelmade, base string cut.

No. 9167, rd. $=7.8-8.0$, bd. $=3.3-3.5$, ht. $=2.2-$ 3.8 , Nile $\mathrm{C} 2$, section not visible, surface uncoated (7.5YR6/3 light brown), wet smoothed, wheelmade, base string cut, intact.

No. 9200, rd. $=10.3-10.6, \mathrm{bd} .=4.0-4.5$, ht. $=$ 2.6-3.7, Nile C2, section not visible, surface uncoated ( 7.5 Y R $/ 4$ brown) with white rim inside and outside (10YR8/2 very pale brown), wheelmade, base string cut, intact, straw visible on the surface.

No. 9207, rd. $=8.2$, bd. $=4.1-4.3$,ht. $=2.5-3.2$, Nile C2, section not visible, surface uncoated (7.5YR6/4 light brown) with white rim inside (10YR8/2 very pale brown), wheelmade, base string cut, intact, straw may be only on the surface.

No. 9211 , rd. $=11.2-11.5$, bd. $=4.2-4.5$, ht. $=3.2-$ 3.6, Nile $\mathrm{C} 2$, section uniform brown, surface uncoated (7.5YR7/4 pink) with white rim inside (10YR8/2 very pale brown), wheelmade, base string cut, intact.

No. 9179, rd.=9.3, bd.=4.7-5.0, ht.= 2.9-3.4, Nile C2, section not visible, surface uncoated (5YR5/4 reddish brown) with white rim inside (10YR8/1 white), wheelmade, base string cut, intact, rim trimmed with tool on top.

No. 9210, rd. $=9.0-9.7$, bd. $=4.0-4.4$, ht. $=1.1-$ 3.1, Nile $\mathrm{C} 2$ + limestone, section not visible, surface uncoated (7.5YR6/4 light brown) with white rim inside (10YR8/1 white), wheelmade, base string cut, intact.

No. 9194, rd.=9.8-10.2, bd.=3.7, ht. $=3.7$, Nile B1, section uniform light brown, surface uncoated (7.5YR7/3 pink), wet smoothed, wheelmade, base string cut.

No. $9180, \mathrm{rd} .=10.2-10.4$, bd. $=3.7$, ht. $=3.1-3.3$, Nile B1, section not visible, surface uncoated (7.5YR6/4 light brown), wheelmade, base string cut, intact, very fine fabric .

No. 9184, rd. $=9.5-9.7$, bd. $=4.1-4.3$, ht. $=5.2-$ 5.6, Nile B1-B2 limestone on surface, section not visible, surface uncoated (7.5YR6/3 light brown), wheelmade, base string cut, intact.

No. 9199, rd.=9.3, bd.=3.4-3.6, ht.= 3.5-4.0, Nile B2, section uniform light brown, surface uncoated (7.5YR6/4 light brown), wheelmade, base string cut, almost complete. 

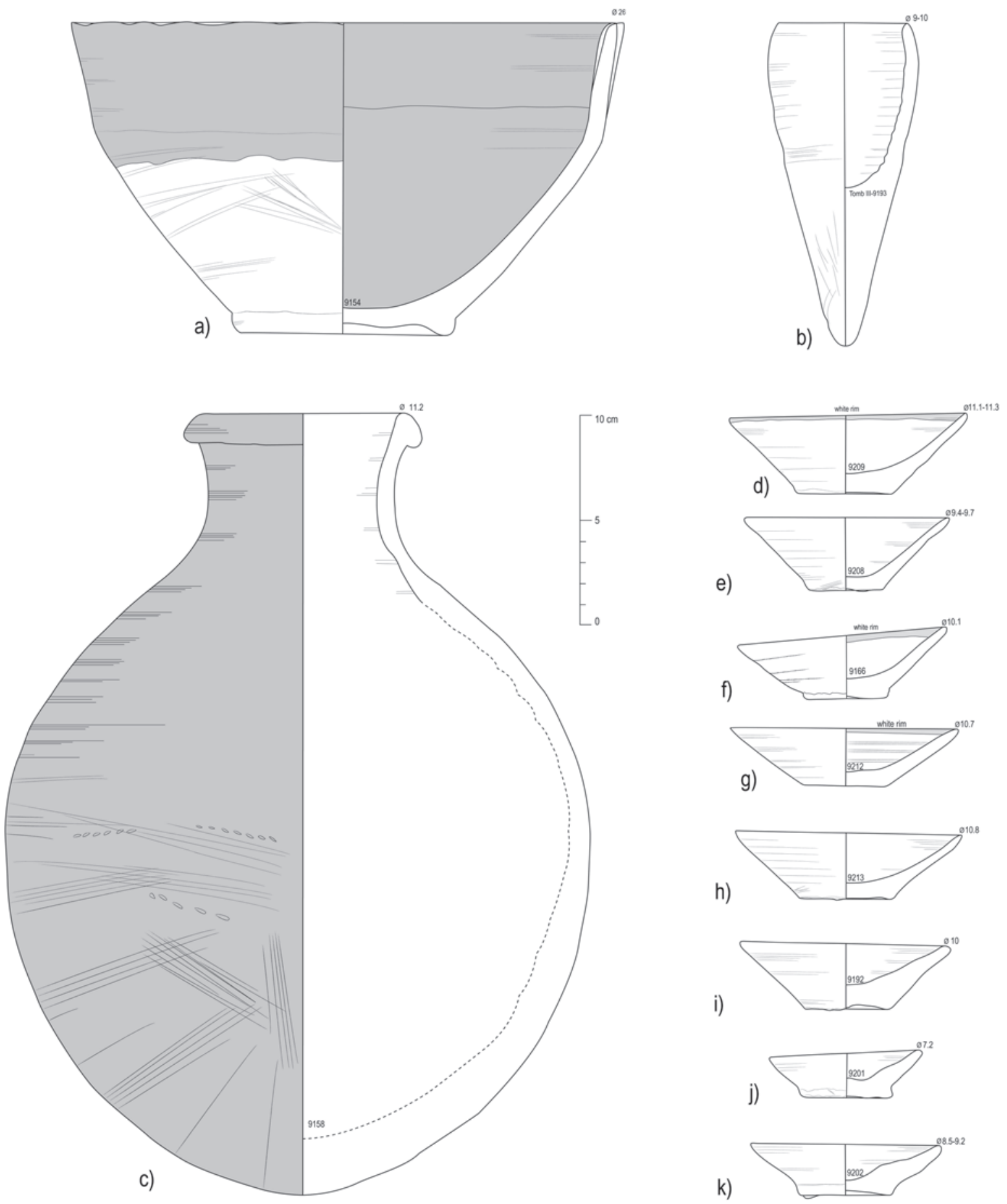

e)

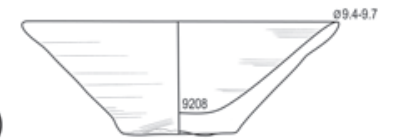

h)

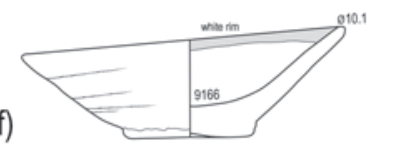

g)
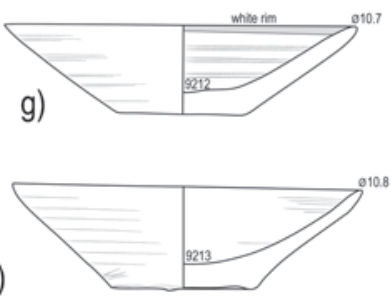

i)

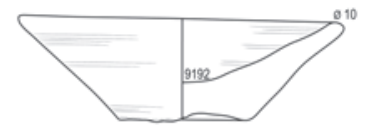

j)

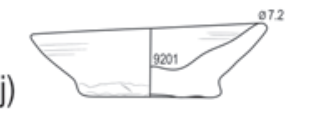

k)
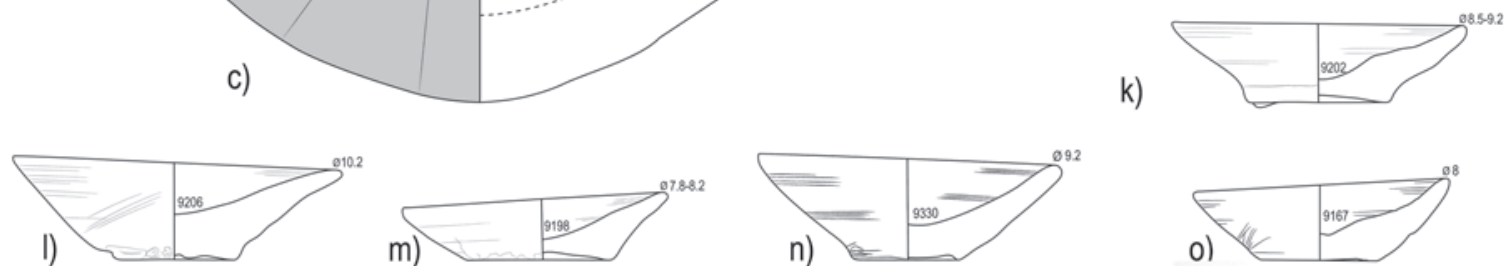

m)
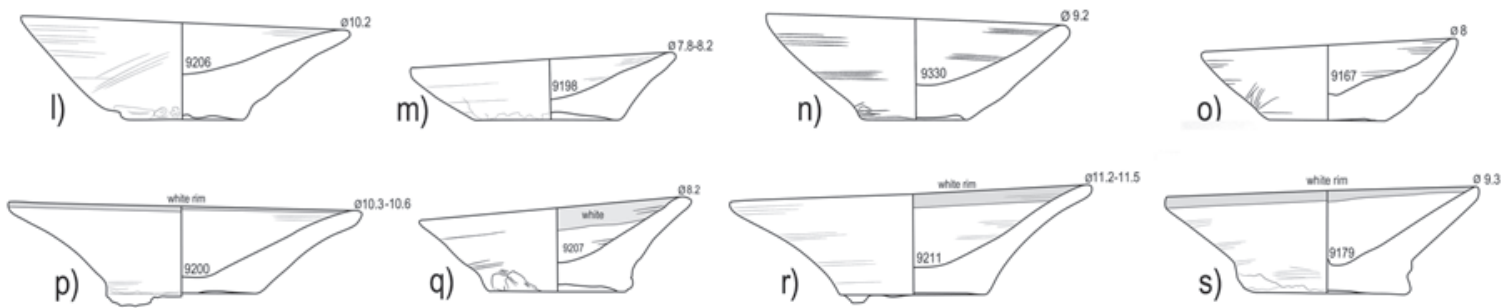

Fig. 10 Pottery from Tomb III-L17, early Middle Kingdom, drawn by B. Bader, inked by E. Schuster. 

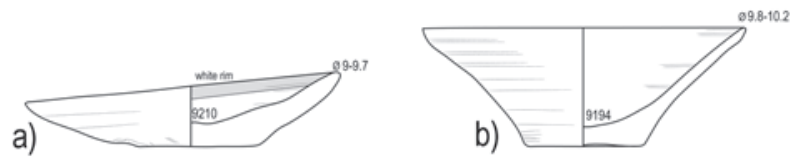

b)

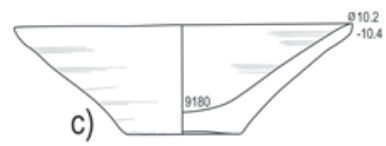

c)

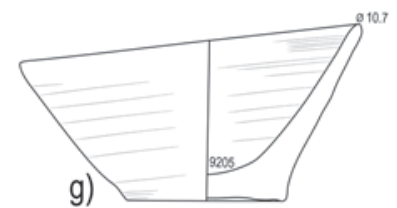

e)
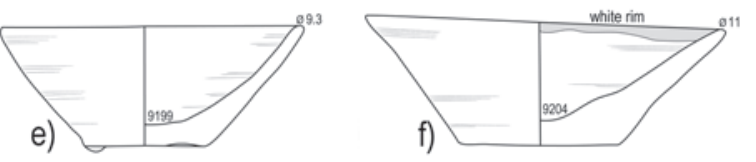

g)

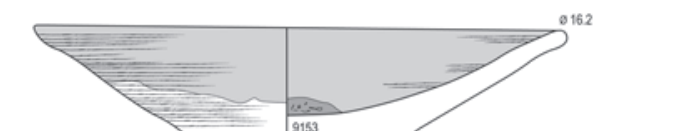

h)
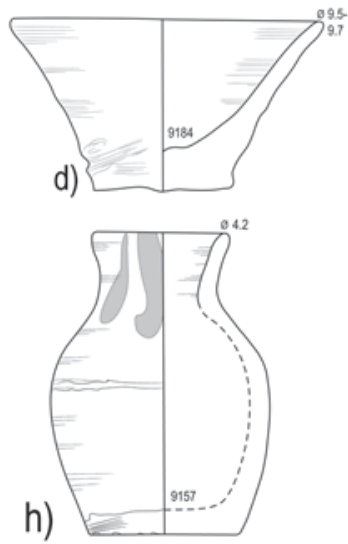

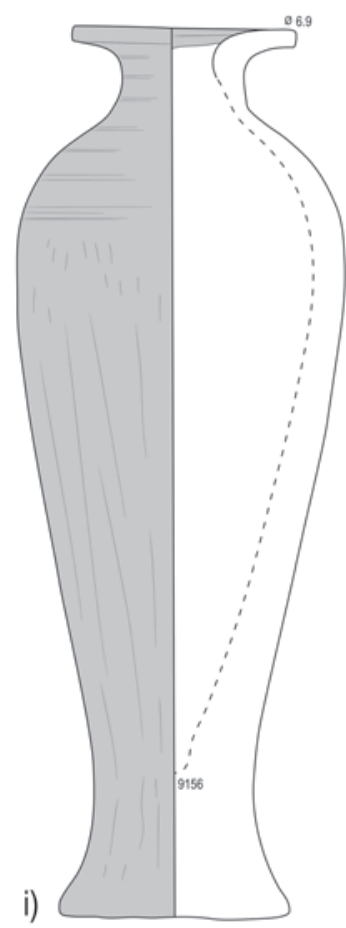

j)
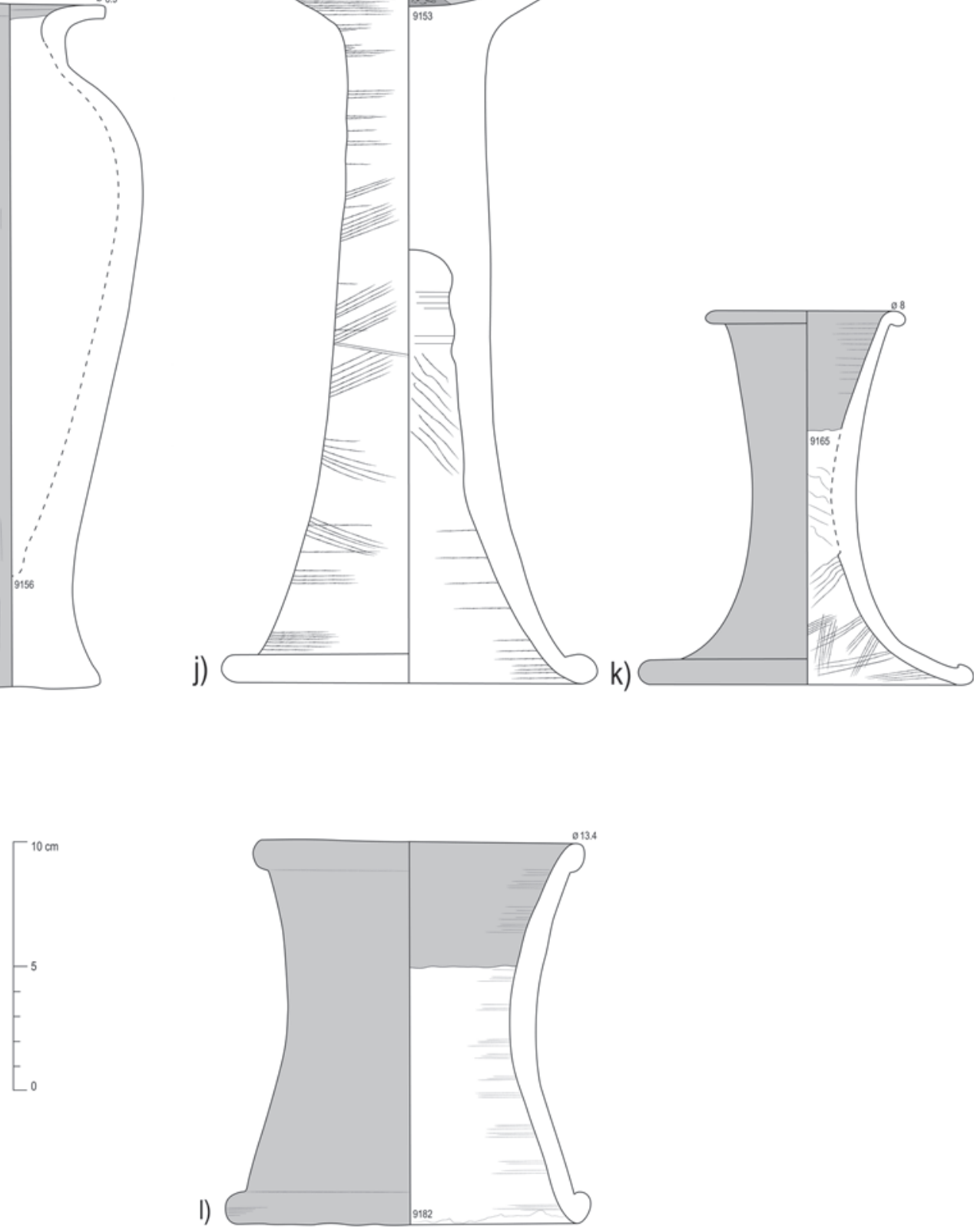

Fig. 11 Pottery from Tomb III-L17, early Middle Kingdom, drawn by B. Bader, inked by E. Schuster. 
No. 9204 , rd. $=11.0$, bd. $=5.0$, ht. $=3.5-4.2$, Nile B2, section uniform brown, surface uncoated (5YR6/6 reddish yellow) with white rim inside (10YR8/1 white), wheelmade, base string cut, almost complete.

No. 9205 , rd. $=10.7$, bd. $=4.8$,ht. $=4.3-5.5$, Nile B2, section uniform brown, surface uncoated (7.5YR6/4-6 light brown to reddish yellow), wheelmade, base string cut.

\section{Nile B1/B2 - Model jar (Fig. 11.h)}

This particular type of model jar with flat base also occurs in another tomb (Fig. 20.1-n). It finds exact parallels only outside of Thebes ${ }^{105}$ and an approximate one in Tomb ' $79 .{ }^{106}$

No. 9157, rd.=4.2, max.d. $=6.7$, bd. $=4.6-4.8$, ht. $=9.4$, Nile B1/B2 fine, section not visible, surface uncoated (7.5YR 5/4 brown), two splashes of red colour on exterior, coiled and turned, base string cut, intact.

\section{Nile B2 - Hes vase (Fig. 11.i)}

The libation vessel has a very rounded shoulder and a narrow outwards flaring foot. The rim is narrower than the shoulder. The manufacture of the piece is very good. Parallels were found in Tomb ' $79^{107}$ and in tombs in Deir el Bahari as well as in the Asasif. Most of them date to the early $12^{\text {th }}$ Dynasty. 108

No. 9156, rd. $=6.9$, max.d. $=10.0$, bd. $=6.9-7.3$, ht.= 27.4, Nile B2, section light brown, surface dark red slipped and polished outside and inside on rim (10R5/6 red) natural surface 7.5YR6/4 light brown, very well smoothed, lower part of vessel handmade, top wheel turned hand modelled, almost complete.

\section{Nile B2 and C2 - Tall stands (Fig. 11. j-l)}

Tall stands occur with a dish attached as well as without a dish on top. Both were presumably used for the same purpose -offering incense or something similar. No. 9153 shows traces of burning some material on the interior of the dish. The other stands differ a little in shape. Whilst no. 9165 has a narrow waist, the other example (no. 9182) is altogether wider. Parallels are found in various places

\footnotetext{
105 Schiestl/SEILER 2012, 980-983.

106 Guidotti 1985, 59, n. 76-78.

107 Guidotti 1985,59 , n. 82, which is a little smaller.

108 Schiestl/SeILer 2012, 508-510.

109 Guidotti 1985, 55, n. 42; 58, n. 70; Schiestl/Seiler 2012, 824-825; Consonni/SESAna 2016, fig. 6.3.
}

in Thebes but they do not particularly help in dating. 109

No. 9153 , rd. $=16.2$, bd. $=14.9$, ht. $=29.6$, Nile $\mathrm{C} 2$ + limestone, section uniform light brown, surface red slipped on interior and on rim exterior (7.5R6/6 light red), natural surface 10YR7/3 very pale brown, wheelmade, the inside of the foot shows no scraping, base wheel turned.

No. 9165 , rd. $=8.0$, bd. $=13.5$, ht. $=15.0$, Nile B2, section not visible, surface red slipped on exterior and on rim on interior, some traces on inside of base (10R5/8 red), natural surface 10YR6/4 light yellowish brown, wheelmade, base scraped on inside, complete.

No. $9182, \mathrm{rd} .=13.4$, bd. $=14.6$, ht. $=15.3-15.5$, Nile B2 + limestone, section uniform light brown, surface thickly dark red slipped and polished (10R5/8 red), natural surface colour 7.5YR5/3 brown, very good quality, wheelmade, base wheelmade.

The pottery from 940-South2-L24 "End of Corridor Deposit" $" 110$ consists of an assemblage of mainly Middle Kingdom and Second Intermediate Period/New Kingdom date. The bulk of the diagnostic material belongs in the Middle Kingdom and it seems to cover two different phases, whilst telltale New Kingdom body sherds and one rim of 'BB-jars' (no. 43, Nile B3) were also present as well as one possible rim of a dish with direct out-turned rim (no. 45, Nile B3, no. 46 Nile B2). An interesting observation on post-depositional processes was made. Some of the reconstructed vessels joined burnt and not burnt material proving that they first broke, were then moved around and only afterwards some of the sherds were burnt by external agents. This feature may suggest more than one intrusion into the tomb with burning incidents. ${ }^{111}$

Because much of the sherd material from 940-South2-L24 duplicates that of L17 above, only selected sherds and complete profiles are shown. The numbering of the fragments follows the pattern of Tomb III-L24-1 to Tomb III-L24-nn, unless a vessel was given an archive number (usually because it was intact or at least recognisable when found).

\footnotetext{
110 Seco Álvarez et al. 2012/13 (appeared 2016), see fig. 13 for the context.

111 The same observation has been made in KV 40, D. Aston, personal communication.
} 


\section{Nile B1 - Hemispherical cups (Fig. 12.a)}

All cup fragments from this context (nos Tomb III-L24-1-8, 24), belong to the wide open cup shape as seen before (Fig. 8.a-d, 9.a-c). Their rim diameters vary from 12.0 to $14.0 \mathrm{~cm}$, whilst their vessel walls can be very delicate but also thick $(0.2-0.4 \mathrm{~cm})$. The direct rims may end rounded or pointed. Only two of the specimens show a thin red rim band, the others were uncoated. The bases, where preserved, were trimmed as is usual for this type. Their appearance is in keeping with those described before.

Tomb III-L24-1, rd.= 14, pres.ht.=1.8, Nile B1, section black, surface burnt, coiled and turned, base trimmed.

Nile B1 and Nile B2 - Carinated cups (Fig. 12.b) Carinated cups appear in a variety of Nile B1 and Nile B2 (Tomb III-L24-20-23, 25), with rim diameters of 8.0 to $14.0 \mathrm{~cm}$. Although the carination is never preserved there is hardly any other way to reconstruct these fragments because their vessel contour runs very straight. The vessels were coiled and turned and well executed. Three fragmentary bases (Tomb III-L24-37-38, 44) may belong with these cups. They were either cut from the hump by knife or irregularly trimmed. ${ }^{112}$

Tomb III-L24-20, $\mathrm{rd} .=14.0$, pres.ht.= 2.7, Nile B1, surface uncoated, coiled and turned.

Nile B1 - Small dish with incurved rim (Fig. 12.c)

Only two examples of this type were found (Tomb III-L24-33-34) and whilst the sherd was very smooth in and out, it was burnt. A red slip is still recognisable. Parallels exist in Egypt but seemingly not in Thebes. ${ }^{113}$

Tomb III-L24-33, $\mathrm{rd} .=9.0$, pres.ht. $=3.4$, Nile B1, surface red slipped on interior and exterior, probably coiled and turned, very well smoothed.

\section{Nile B2 - Shallow dishes with trimmed rim (Fig. 12.e)}

Several examples of this type were found (Tomb III-L24-27-31). The direct rim is thickened at the end and was trimmed with a tool or with the fingers of the potter. The rim diameters vary from 19.0 to $24.0 \mathrm{~cm}$. Some of the fragments may belong to the same vessels. Some of the sherds

\footnotetext{
112 Schiestl/Seiler 2012, 227-228, no 9 to 22, 241-242, no 22 to 38 .

113 SChiestl/Seiler 2012, 928-929.
}

were red slipped only on the interior, one on the interior and the rim on the exterior.

Tomb III-L24-27, rd.= 19, pres.ht.= 7.1, Nile B2 + lime, burnt interior/exterior, surface probably red slipped,

Nile B2 - Bowl with undulating rim (not shown) Two fragments of this type were found in the assemblage, but the preservation was not good enough to obtain a reasonably certain rim diameter (Tomb III-L24-35-36). They are probably similar to undulating dishes shown in the Middle Kingdom Handbook. ${ }^{114}$

\section{Nile B2 - Medium carinated bowl (Fig. 12.f)}

One rim and one base fragment may belong together (Tomb III-L24-39-40) to form a medium carinated bowl. It was burnt. The manufacturing technology and the general feel of the ware seemed to justify the current reconstruction. ${ }^{115}$

Tomb III-L24-39-40, rd.= 18.3, bd.=4.4, reconstr.ht. $=6.3+3.8$, Nile B2 + lime, section black, surface burnt, coiled and turned, base trimmed.

Nile B2/C1 - Medium carinated bowl with undulating rim (Fig. 12.g)

This type has already been discussed (see Fig. 8.f, 10.a).

No. 9197, rd. $=22.5-22.8, \mathrm{bd} .=8.0$ ht. $=11.4$, Nile B2/C1, some larger straw on the surface, section not visible, surface red slipped on interior and exterior below carination (10R6/6 light red), natural surface colour 7.5 YR5/4 brown, coiled and turned, disc base with low ring, separate pad of clay added by hand, intact.

\section{Nile C2 - Medium dishes (Fig. 12.d)}

Several examples of dishes with direct out-turned rim made of the rough Nile $\mathrm{C} 2$ were found (Tomb III-L24-47-52). The assignment to the Middle Kingdom was clear by the material and by the technology (very rough scraping patterns close to the base). The rim diameters range from 15.0 to $20 \mathrm{~cm}$. Dishes without obscured surface by burning, had been left uncoated. No close dating of this long lived type is possible.

Tomb III-L24-48, rd.= 19.0, pres.ht. $=2.2$, Nile $\mathrm{C} 2$, surface uncoated, coiled and turned, base scraped.

\footnotetext{
114 Schiestl/SeIler 2012, 82-83.

115 Schiestl/Seiler 2012, 237, no 2.
} 

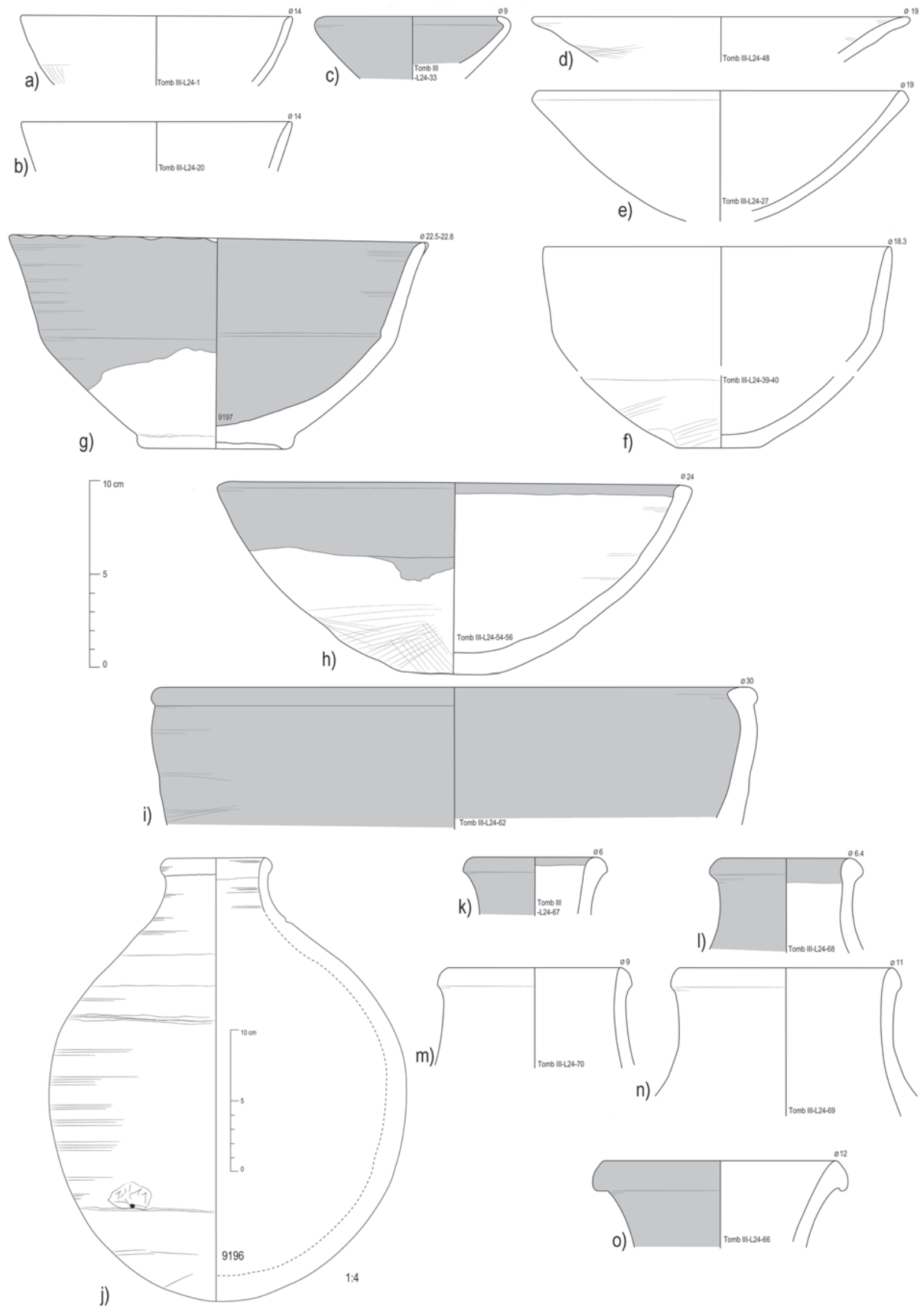

Fig. 12 Pottery from Tomb III-L24, early Middle Kingdom, drawn by B. Bader, inked by E. Schuster. 
Nile C2 - Medium to large bowl (Fig. 12.h)

Medium to large bowls with thickened and trimmed rims belong to the assemblage (Tomb IIIL24-52-57, 59-60). Their rim diameters range from 24.0 to $28.0 \mathrm{~cm}$. The intact examples are always asymmetrical and therefore the rim sherds can only be measured with a diameter chart with limited accuracy. The surface of the fragments may be uncoated or red slipped on the top part of the vessels (wide rim band on exterior and narrower on the interior). A red slip, which may have an orange tinge (5YR6/6 reddish yellow, for example) was observed. An exact parallel does not exist in the Middle Kingdom Handbook, although raw material and manufacturing technology place this bowl in this period.

Tomb III-L24-54-56, rd. $=24.0$, ht. $=10.3$, Nile C2, surface wide red slipped band (2.5YR5/6 red) on uncoated exterior (10YR6/4 light yellowish brown), narrow red slipped band on uncoated interior, coiled and turned, base scraped, smooth on the interior with the stain of a wick.

\section{Nile C2 - Large restricted bowl with incurved} rim (Fig. 12.i)

Several fragments of a large bowl or basin were red slipped on the interior and the exterior (Tomb III-L24-61-65). Some of the sherds were burnt. The top of the rim is slightly incurved with a somewhat irregularly folded lip. The technology follows the usual pattern of coiling and turning. The better preserved sherds show traces of rough scraping. ${ }^{116}$

Tomb III-L24-62, $\mathrm{rd} .=30.0$, pres.ht. $=7.3$, Nile $\mathrm{C} 2$, surface red slipped on interior and exterior, coiled and turned, base scraped.

\section{Nile C2 - 'Beer' jars (Fig. 12.j-0, 13.a)}

At least two different types of beer jars were found in this assemblage that belong to different chronological phases, known from other sites by analogy only. The older one has a very globular body (Fig. 12.j) with a short straight neck and a thickened lip. External parallels date this type to the beginning of the $12^{\text {th }}$ Dynasty. ${ }^{117}$ At Thebes such jars have been found in a Middle Kingdom tomb below the Mortuary temple of Amenhotep II. ${ }^{118}$ Some rim fragments can be connected with this globular type (Fig. 12.k-1). The other, later type usually occurs from the late $12^{\text {th }}$ Dynasty onwards to the early Second Intermediate Period, ${ }^{119}$ is more ovoid in body shape (Fig. 13.a) and the rim fragments are funnel shaped with a folded lip (Fig. 12.o). Fig. 12. $\mathrm{m}-\mathrm{n}$ show a wider rim diameter, which might mean they represent a chronological development. This hypothesis still needs to be tested.

\section{Earlier type}

No. 9196, $\mathrm{rd} .=6.8$, max.d. $=25.0, \mathrm{ht} .=31.2$, Nile C2 + limestone, section not visible, surface red slip on exterior and interior on rim (10R5/6 red), very well smoothed, coiled and turned, base scraped, intact, possible traces of mud stopper on the shoulder, possible killing hole in the body

Tomb III-L24-67, rim, rd.= 6.0, pres.ht. $=3.1$, Nile C2, surface red slipped on exterior and on rim interior, coiled and turned.

Tomb III-L24-68, rim, rd.= 6.4, pres.ht. $=5.1$, Nile C2 + limestone, surface red slipped on exterior and on rim interior, coiled and turned.

\section{Later type}

Tomb III-L24-69 rim, rd.= 11.0, pres.ht. $=7.9$, Nile $\mathrm{C} 2+$ limestone, surface uncoated, coiled and turned.

Tomb III-L24-70 rim, rd. $=9.0$, pres.ht. $=5.4$, Nile C2 + limestone, surface probably uncoated burnt, coiled and turned.

Tomb III-L24-58, ovoid base, pres.ht.= 11.6, Nile C2, surface uncoated, held together during drying by rope, coiled and turned, base scraped (Fig. 13.a).

Tomb III-L24-66, rim, rd.= 12.0, pres.ht.=4.6, Nile $\mathrm{C} 2+$ limestone, surface red slipped on exterior, coiled and turned.

\section{Nile B1 - Model dishes and model bowls (Fig. 13.b-c)}

Two examples of small dishes made from a very finely levigated clay were present in this context (Tomb III-L24-15, 16). Their rim diameter is 10.0 and $7.0 \mathrm{~cm}$, respectively. The latter is deeper ${ }^{120}$ than the other and often on the interior the edges of the coils are visible and not smoothed away. This trait can also be observed in the material

\footnotetext{
119 Seiler 2005, 71, Abb. 24, ZN 02/162, DAN 1.

120 Schiestl/Seiler 2012, 875, no 6, late $12^{\text {th }}$ Dynasty.
}

\footnotetext{
117 ARNOLD 1988, 130-132.

118 Consonni/Sesana 2016, fig. 3.
} 

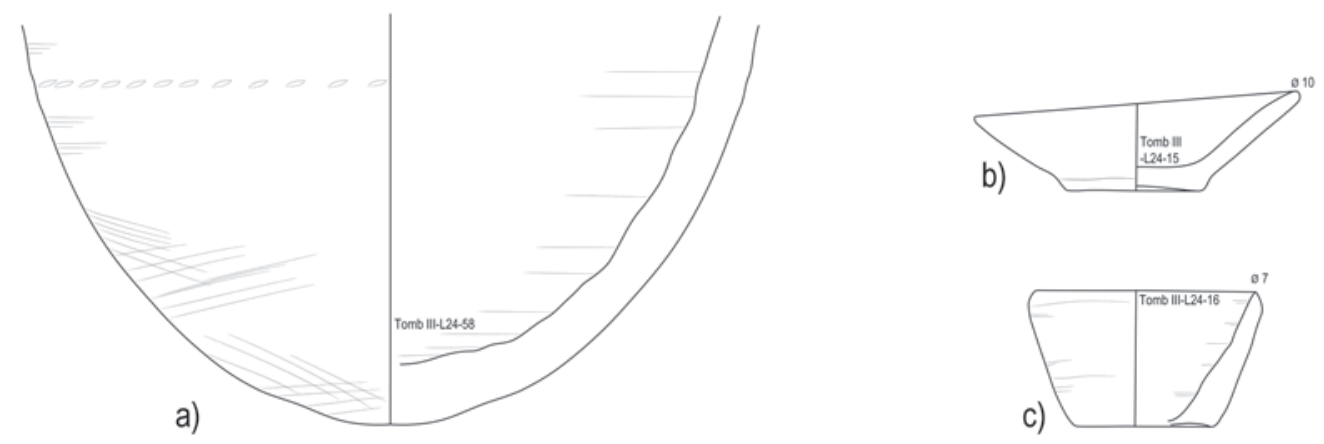

d)

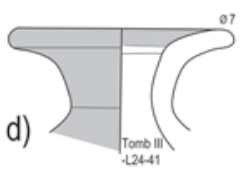

e)
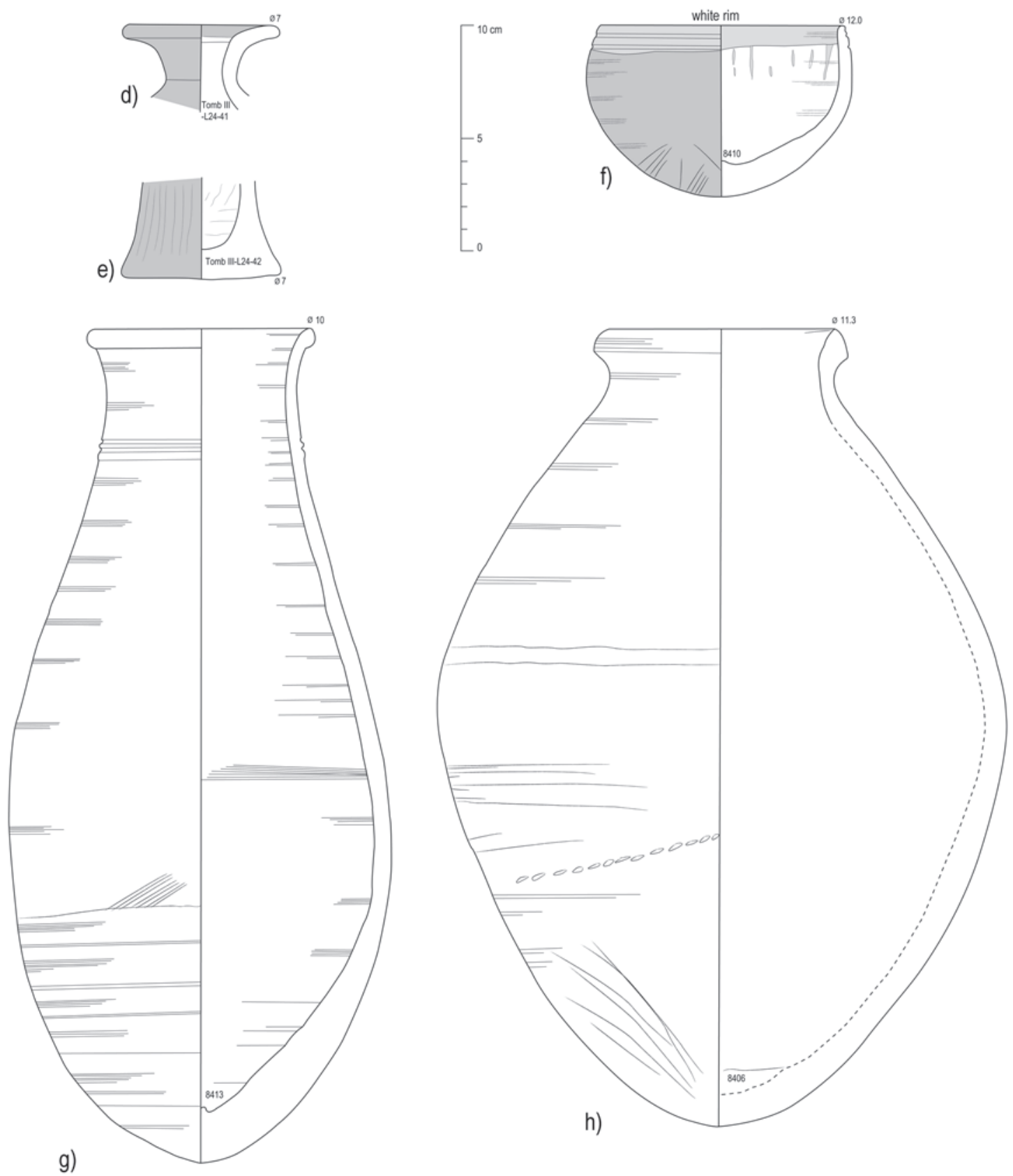

Fig. 13 a-g) Pottery from Tomb III-L24 Middle and New Kingdoms, h) Pottery from Tomb III-L38, late Second Intermediate Period/New Kingdom, drawn by B. Bader, inked by B. Bader and E. Schuster. 
found in the late Middle Kingdom tomb of the lady Senebtisi at Lisht. ${ }^{121}$ One other dish fragment (Tomb III-L24-19) was made from Nile B2, whilst another (Tomb III-L24-14) consisted of the rough Nile C2 fabric. All bases of these small vessels were string cut.

Tomb III-L24-15, rd. $=10.0$, bd. $=4.2$, ht $=3.1-$ 2.3, Nile B1, surface uncoated (7.5YR6/4 light brown), coiled and turned, base string cut.

Tomb III-L24-16, rd.= 7.0, bd.= 5.0, ht.= 4.1, Nile B1, surface uncoated (10YR6/3 pale brown), coiled and turned, base string cut, top of rim trimmed with a tool.

\section{Nile B1 - Model jars (not shown)}

Several diagnostic fragments of model jars are derived from this context (Tomb III-L24-9-13). Most of them were made from Nile B1 and burnt. Those not burnt were well made and uncoated as well as wet smoothed. The rim diameters are consistently $4.0 \mathrm{~cm}$ and the vessel shape of the fragments resembles very closely that of no. 9157 in Fig. 11.h, from Tomb III-L17.

\section{Nile B3/D - Hes vase (Fig. 13.d-e)}

The rim and base fragment of a Hes vase rounds off the repertoire of this context (Tomb IIIL24-41-42). The Nile clay fabric contains abundant limestone particles and also some fine chaff. Due to its incomplete state no parallels can be cited because height and contour of the shoulder cannot be reconstructed. It is unlikely to have had a spout because there seemed to be not enough space $(80 \%$ preservation of the rim).

Tomb III-L24-41, rd.= 7.0, pres.ht. $=2.9$, Nile $\mathrm{B} 3 / \mathrm{D}$, surface red slipped on exterior and on rim interior, partly burnt, coiled and turned.

Tomb III-L24-42, bd.= 7.0, pres.ht.= 4.2, Nile $\mathrm{B} 3 / \mathrm{D}$, surface red slipped on exterior, coiled and turned, base trimmed, exterior of foot vertically trimmed.

Later pottery forms dated to the late Second Intermediate Period and the New Kingdom attest to subsequent use of the tomb after the original burial(s). Interestingly most of the intrusive material was found at the end of the corridor.

\footnotetext{
121 Mace/Winlock 1916, 112.

122 SeILER 2005, 90-91, but deeper.

123 Aston 2003, 140-149, Phases I-II, Thutmosis III-IV.

124 Seco Álvarez et al. 2012/13 (appeared 2016), plan 9, fig. 14 , the anthropological report $339-342$, the pot is pub-
}

\section{Nile B2/C1 - Bowl with incised grooves (Fig. 13.f)}

Such bowls with incised grooves and a white rim band over a red slip are known from Second Intermediate Period contexts at Dra Abu el-Naga. ${ }^{122}$

No. $8410, \mathrm{rd} .=11.0-12.0, \mathrm{ht} .=7.5$, Nile B2/C1 + limestone, section not visible, surface red slipped on exterior (2.5YR5/6 red) with a white rim (10YR8/2 very pale brown) natural colour 5YR6/4 light reddish brown, wheelmade, base trimmed with a tool.

\section{Nile B2 - Tall slender jar (Fig. 13.g)}

This type of tall jar is securely dateable into the New Kingdom by its typical elongated and slender shape, the material as well as the manufacturing technology. ${ }^{123}$

No. $8413, \mathrm{rd} .=10.0$, max.d. $=16.6$, ht. $=36.2$, Nile B2, section dark grey core with red and brown oxidation zones, surface uncoated (2.5YR6/4 weak red), wheelmade, base wheelmade: turned on the wheel and scraped horizontally.

In addition four mud stoppers were found which were very crumbly. Only the first one was fired or somehow otherwise compacted.

Finally the only vessel found in the burial chamber 940-South2 L38, which contained four bodies, suggests a date in the late Second Intermediate period or the early New Kingdom. ${ }^{124}$

\section{Nile B2 - Large jar (Fig. 13.h)}

No. 8406 , rd.= 11.3, max.d. $=25.3$, ht. $=35.4$, Nile B2, section not visible, surface red slipped on exterior (2.5YR6/6 red), natural surface colour 7.5 YR6/2 pinkish gray, very well smoothed, wheelmade (W1), base wheelmade, intact.

\subsection{3.) Tomb IV (square 980 -South3) ${ }^{125}$}

Tomb IV excavated in 2010 contained two assemblages of pottery, one in the corridor (980S3L1) and one in the burial chamber (980S3L2). Neither group is homogenous. The corridor deposit included material of the late Middle Kingdom and the New Kingdom, whilst the burial chamber group consisted of vessels belonging to the early Middle

lished in fig. 37.b; SeILer 2005, Falttafel 4.2, the max.d. of our vessel sits higher up the vessel height.

125 Archaeological description Seco Álvarez et al. 2012/13 (appeared 2016), 338, plan 8. 
and New Kingdom corpus. This may mean that three use phases are to be expected or at least two with some activity in the New Kingdom. The end of the accessibility of Tomb IV may be expected at the beginning of, or during the building project of Thutmosis III.

The ceramic material from the corridor deposit (980S3L1) dating to the Middle Kingdom will be listed first and the New Kingdom material afterwards. Most of the types were already discussed before.

\section{Nile B1 - Carinated cup/dish (Fig. 14.a)}

This fragment belongs to the Middle Kingdom corpus. ${ }^{126}$

Tomb IV-L1-4, rd.= 16.0, pres.ht.=4.7, Nile B1, surface red slipped on interior and exterior (10R5/3-4 weak red), wheel turned, very fine.

\section{Nile B1 - Ring base of closed vessel (Fig. 14.b)}

Tomb IV-L1-10, bd.=8.6, pres.ht.= 5.9, Nile B1, surface dark red slipped on exterior? smoke blackened, wheel turned, well smoothed, ring added separately.

\section{Nile B2/C1 - Dish with direct out-turned rim} (Fig. 14.d)

This type was already discussed.

Tomb IV-L1-5, rd.=24.0, pres.ht.=2.3, Nile B2/ $\mathrm{C} 1$, surface uncoated (10YR7/3 very pale brown), coiled and turned, fine quality, burnt on rim.

\section{Nile B2/C1 - Base of pointed beaker (not shown)}

This type was already discussed, only a base fragment belongs to this context.

Tomb IV-L1-6, pres.ht. $=10.0$, Nile $\mathrm{B} 2 / \mathrm{C} 1$, uncoated (7.5YR5/4 brown), base hand formed, vertically trimmed and smoothed. (early type with real point) not drawn.

\section{Nile C2 - 'Beer' jar (Fig. 14.c, e)}

The body of the vessel is ovoid, while the rim fragment belongs to the kettle rim type with a short neck. ${ }^{127}$

Tomb IV-L1-8, rd. $=9.0$, pres.ht. $=8.0$, Nile C2 with some lime, surface uncoated (7.5YR7/6 reddish yellow), coiled and turned.

\footnotetext{
126 Schieste/Seiler 2012, 227-228, 242.

127 SeILer 2005, 70-75, DAN 3.
}

Tomb IV-L1-9, pres.ht. $=14.0$, Nile C2, surface uncoated (7.5YR6/6 reddish yellow), coiled and turned, base scraped on exterior, but very well smoothed also inside.

\section{Marl A2 -Base of conical vessel (Fig. 14.f)}

In the ceramic material of the mortuary temple of Thutmosis III conical vessels, mostly made from Marl clay fabrics in two wares (uncoated; red slipped and vertically burnished) appeared quite frequently. Several such vessels have been found in Middle Kingdom Tomb contexts but rarely if at all in other temple contexts, not even on the surface. Whilst it was at first unclear whether this vessel type might be modern, because no published parallels could be found, there are similar vessels in the Metropolitan Museum of Art in New York, from Winlock's Theban Tomb 703, which were more obviously coiled and not vertically scraped. The area, in which these pottery vessels were found is practically immediately east of the Thutmosis III temple. ${ }^{128}$

Tomb IV-L1-7, bd. $=8.9$, pres.ht. $=10.8$, Marl A2, scum (10YR8/2 very pale brown), base coiled and turned (on wheel?), vertically scraped on exterior, very fine, very hard with convex base, would not stand on an even surface. It was squeezed together as the folds on the interior show.

\section{Nile B1 - Model dishes (Fig. 14.g-h)}

This type was already discussed.

Tomb IV-L1-1, rd.= 7.8-8.0; bd.= 4.0-5.0, ht. $=3.4-4.5$, Nile B1, surface uncoated $(5$ YR6/4 light reddish brown), wheel turned, base string cut, asymmetrical.

Tomb IV-L1-2, rd.=8.0, bd. $=5.0$, ht. $=3.3$, Nile B1, surface uncoated (10YR6/4 light yellowish brown), wheel turned, base string cut.

\section{Nile B1 - Model jar (Fig. 14.i)}

This vessel type belongs to the Middle Kingdom corpus. ${ }^{129}$

980-S3-L1-3, rd. $=4.5$, bd. $=2.3-2.8$, ht. $=5.6$, Nile B1 with limestone, surface uncoated (10YR6/3 pale brown), wheel turned, base string cut, asymmetrical.

\footnotetext{
128 Metropolitan Museum nos 35.3.287-292, heights $48.5 \mathrm{~cm}$, $40.0 \mathrm{~cm}, 26.0 \mathrm{~cm}, 25.5 \mathrm{~cm}, 24.5 \mathrm{~cm}$ and $23.0 \mathrm{~cm}$. Dated from $11^{\text {th }}$ to $18^{\text {th }}$ Dynasty in online database http://www. metmuseum.org/art/collection/search/.

129 Schiestl/Seiler 2012, 977, throughout the $12^{\text {th }}$ Dynasty.
} 

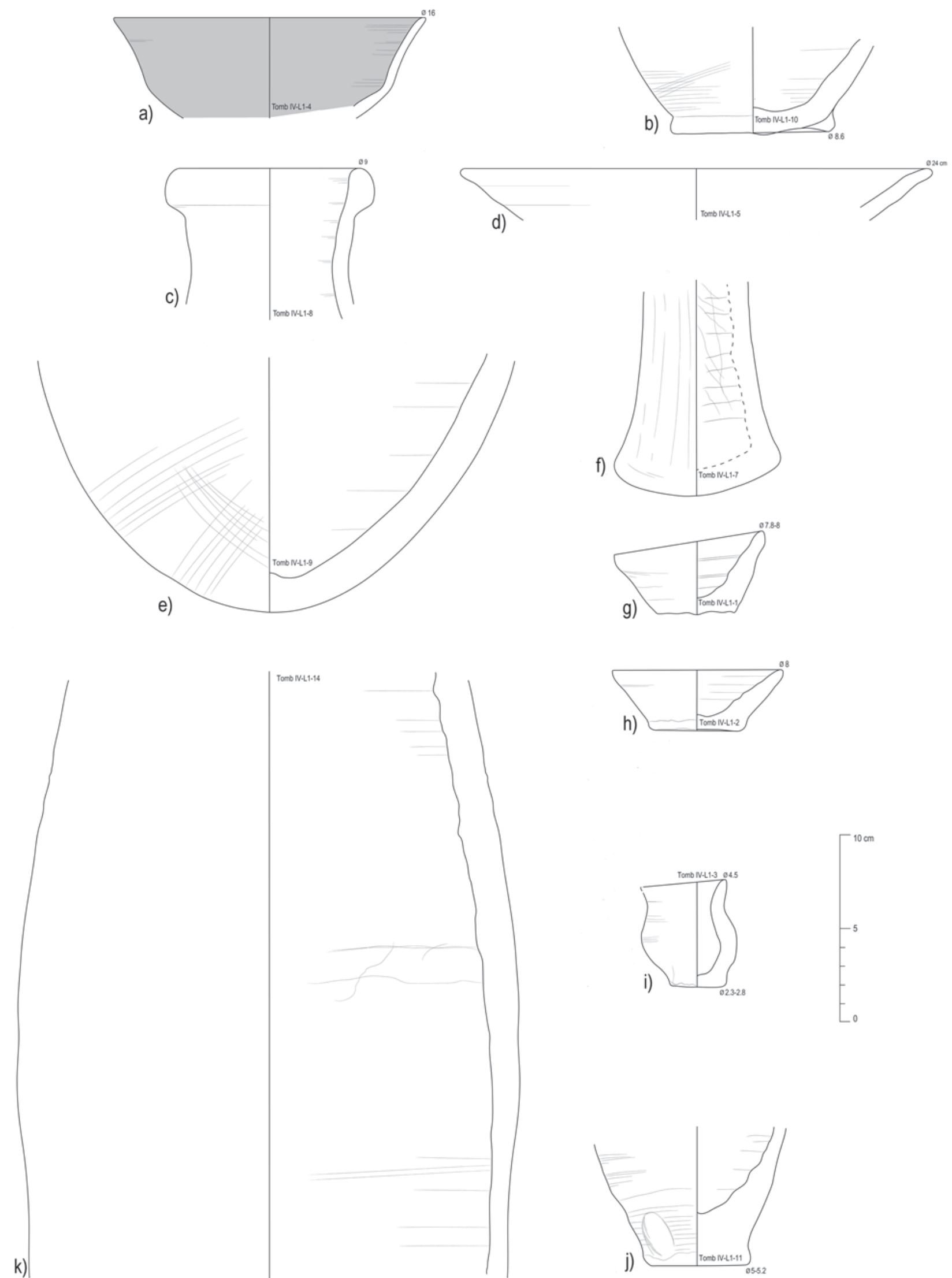

h)

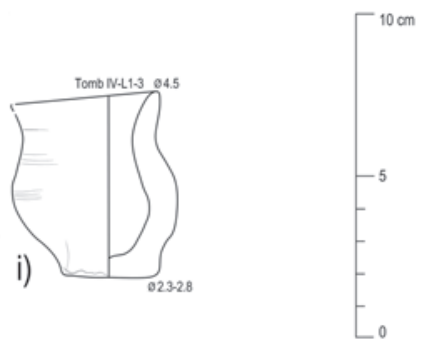

Fig. 14 Pottery from Tomb IV-L1, Middle and New Kingdom, drawn by B. Bader, inked by E. Schuster. 


\section{New Kingdom pottery}

Nile B3 - Flat Base of closed vessel (Fig. 14.j)

This vessel fragment is reminiscent of the New Kingdom technology for mass producing medium sized 'BB jars', with string cutting and finger impressions on the exterior. The base diameter appears too small for such a vessel, but smaller vessels with different contours do exist (see Fig. 42.d-f).

Tomb IV-L1-11, bd. $=5.0-5.2$, pres.ht. $=6.7$, Nile B3, surface uncoated (wet smoothed?, 5YR6/6 reddish yellow), wheel turned, base string cut with deep finger impressions.

\section{Marl A4 - Large closed vessel (Fig. 14.k)}

This wheelmade vessel fragment must have belonged to a fairly large vessel, perhaps for storage.

Tomb IV-L1-14, pres.ht. $=32.0$, Marl A4, uncoated (scum, 2.5Y8/2 pale yellow), wheel turned with join line. On the interior the surface seems to be covered with red slip or stained from contents (accidential?).

\section{Five mud stoppers}

Three stoppers were lentil shaped and very crumbly. Impressed rim diameters range from ca $10.0 \mathrm{~cm}$ to $11.0 \mathrm{~cm}$. One stopper is lentil shaped but flattened on the 'base' with the orifice impression of a diameter of 9.0 to $10.0 \mathrm{~cm}$. One mud stopper is very small with an impression of 2.0 to $3.0 \mathrm{~cm}$ wide orifice of a vessel. Only the model jar is remotely fitting this description in this assemblage.

\section{S3L2 Burial chamber deposit}

The Middle and New Kingdom ceramic material is discussed according to shape in this small assemblage.

\section{Nile B1 - Hemispherical cups (Fig. 15.a-c)}

The hemispherical cups of this context are all of a wide open form with a rim diameter from 11.6 to $12.6 \mathrm{~cm}$. One cup was well enough preserved to calculate the vessel index of 187 . None of these cups showed the thin red rim band. All of them were very thin walled and well executed. ${ }^{130}$

Tomb IV-L2-1, rd.= 12.6, pres.ht.=4.1, Nile B1, surface uncoated (7.5YR6/4 light brown), coiled and turned.

\footnotetext{
130 Schiestl/SEILER 2012, 96-99.

131 Schiestl/Seiler 2012, 227-228.

132 SChiestl/Seiler 2012, 982-984.
}

Tomb IV-L2-2, rd.= 12.0, pres.ht. $=5.8$, Nile B1, surface uncoated (7.5YR6/6 light brown), coiled and turned, base trimmed.

Tomb IV-L2-3, rd.= 11.6, ht. $=6.0-6.6$, Nile B1, surface uncoated (7.5YR6/6 light brown), coiled and turned, base trimmed, very well smoothed, vessel index 187.

\section{Nile B1 fine - Wheelmade ring base of open vessel (Fig. 15.d)}

This fragment could have belonged to a carinated cup or another delicate open vessel type of fine material. ${ }^{131}$

Tomb IV-L2-7, bd. $=4.5-4.8$, pres.ht. $=3.8$, Nile B1 fine, surface dark red slipped on interior (10R5/6 red), uncoated on exterior (7.5YR5/4 brown), coiled and turned, base (wheel?) turned.

\section{Nile B1 - Model jar (Fig. 15.f)}

Comparable vessels have only been found outside of Thebes. The quality of it is very good. ${ }^{132}$

Tomb IV-L2-8, rd.= 4.4, max.d.= 7.6, bd.=4.6, ht. $=9.3$, Nile B1, uncoated (7.5YR6/4 light brown), vessel covered in white substance, coiled and turned, base string cut.

\section{Nile B2 - Hemispherical cup (Fig. 15.g)}

The only example of the slightly rougher version of cups in this context is slightly deeper than those of Nile B1 and the rim was adorned with a red rim band. The vessel index is also a little lower, 167. It is doubtful that this difference has a chronological significance here. ${ }^{133}$

Tomb IV-L2-4, rd.= 12.0, ht. $=7.5$, Nile B2, surface uncoated (7.5YR6/4 light brown) with thin red rim band (10R6/6 red), coiled and turned, base trimmed, vessel index 167 .

\section{Nile B2/C1 - Chalice(?) base (Fig. 15.e)}

Chalices range in date from the $11^{\text {th }}$ to the early $12^{\text {th }}$ Dynasty. One early example is very similar. ${ }^{134}$

Tomb IV-L2-6, bd.= 5.2, pres.ht.= 2.1, Nile B2/ $\mathrm{C} 1$, surface red slipped on interior and exterior (also under base), coiled and turned.

\section{Nile B2/C1 - Incurved dish (Fig. 15.h)}

This vessel type does not fit into the Middle Kingdom assemblage and may belong to a later use

\footnotetext{
133 Schiestl/Seiler 2012, 96-99.

134 Schiestl/Seiler 2012, 296-297, best match is 297, no 1; Consonni/Sesana 2016, fig. 6.10.
} 


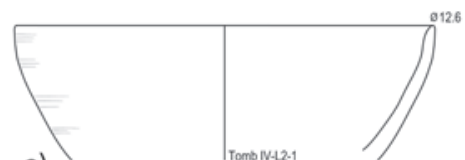

a)

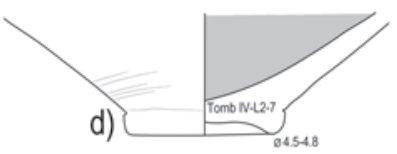

e)

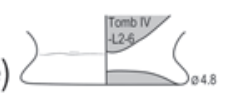

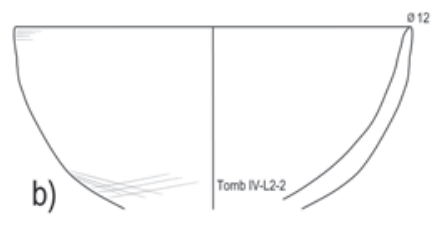

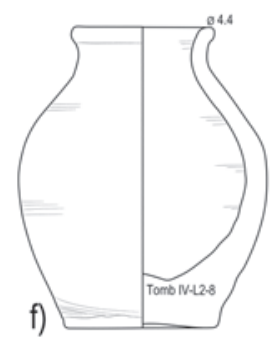

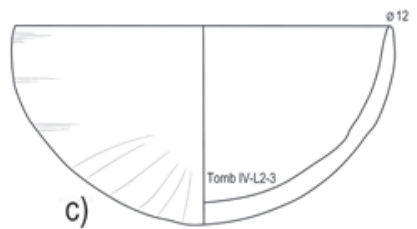

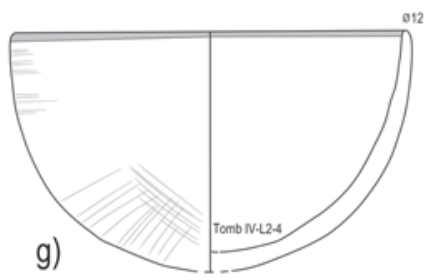

g)

h)
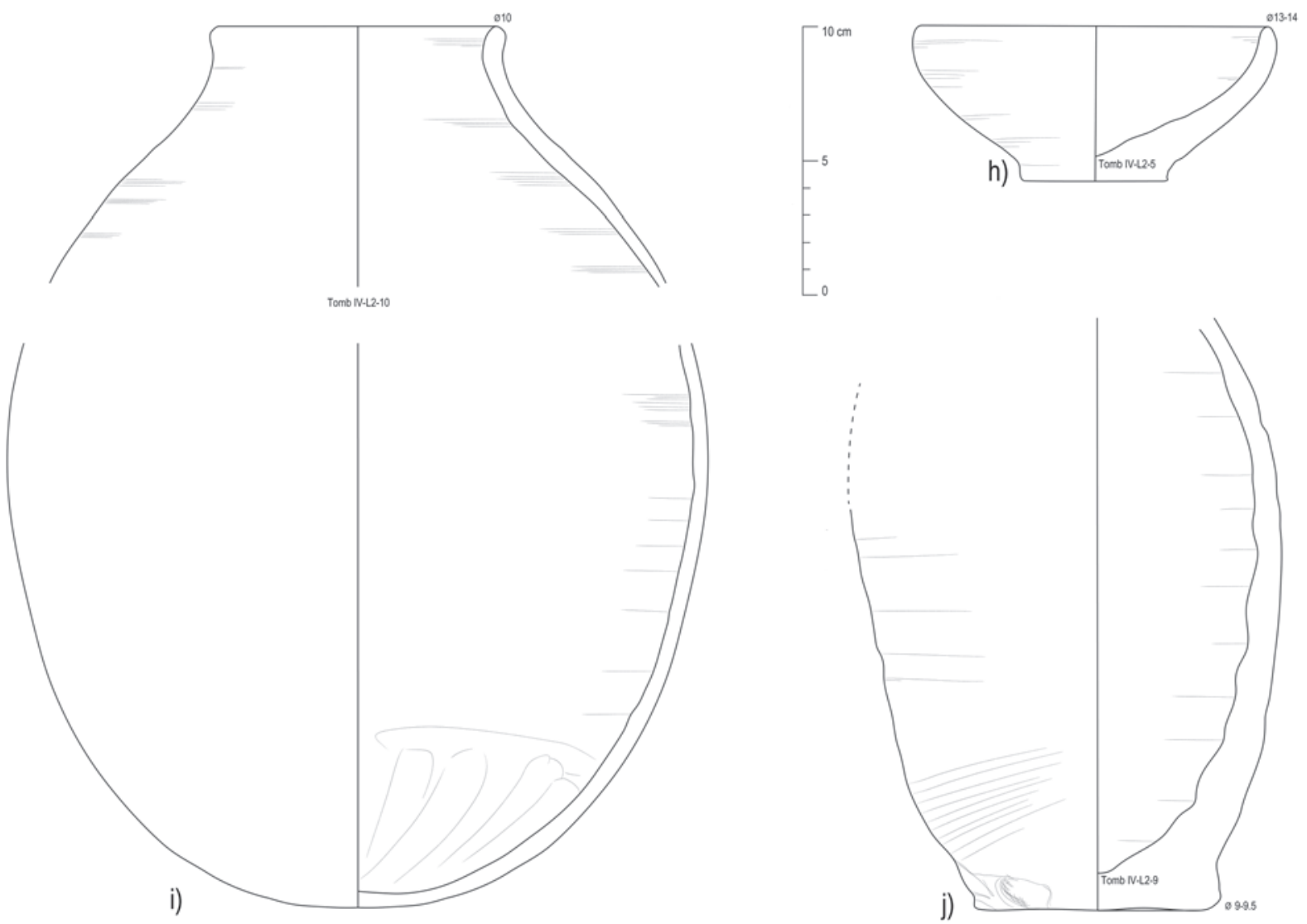

Fig. 15 Pottery from Tomb IV-L2, Middle and New Kingdom, drawn by B. Bader, inked by E. Schuster.

phase of the tomb as it does not appear in the Middle Kingdom Handbook.

Tomb IV-L2-5, rd.= 13.0-14.0, bd.= 5.4, ht.= 5.8 , Nile $\mathrm{B} 2 / \mathrm{C} 1+$ limestone, surface covered in white layer (natural surface 7.5YR5/4 brown), coiled and turned, well smoothed, base string cut, vessel index 235 .

\section{Nile C2 - 'Beer' jar (Fig. 16)}

This beer jar type belongs clearly to the earlier globular shape already seen in Tomb III. This example shows a direct vertical rim without any thickening or lip, for which no exact parallel is available in the Middle Kingdom Handbook in this size. ${ }^{135}$ Material and manufacture reinforce this identification, though.

135 Consonni/Sesana 2016, Fig. 3; Schiestu/Seiler 2012, 406-413 both show folded rims. 


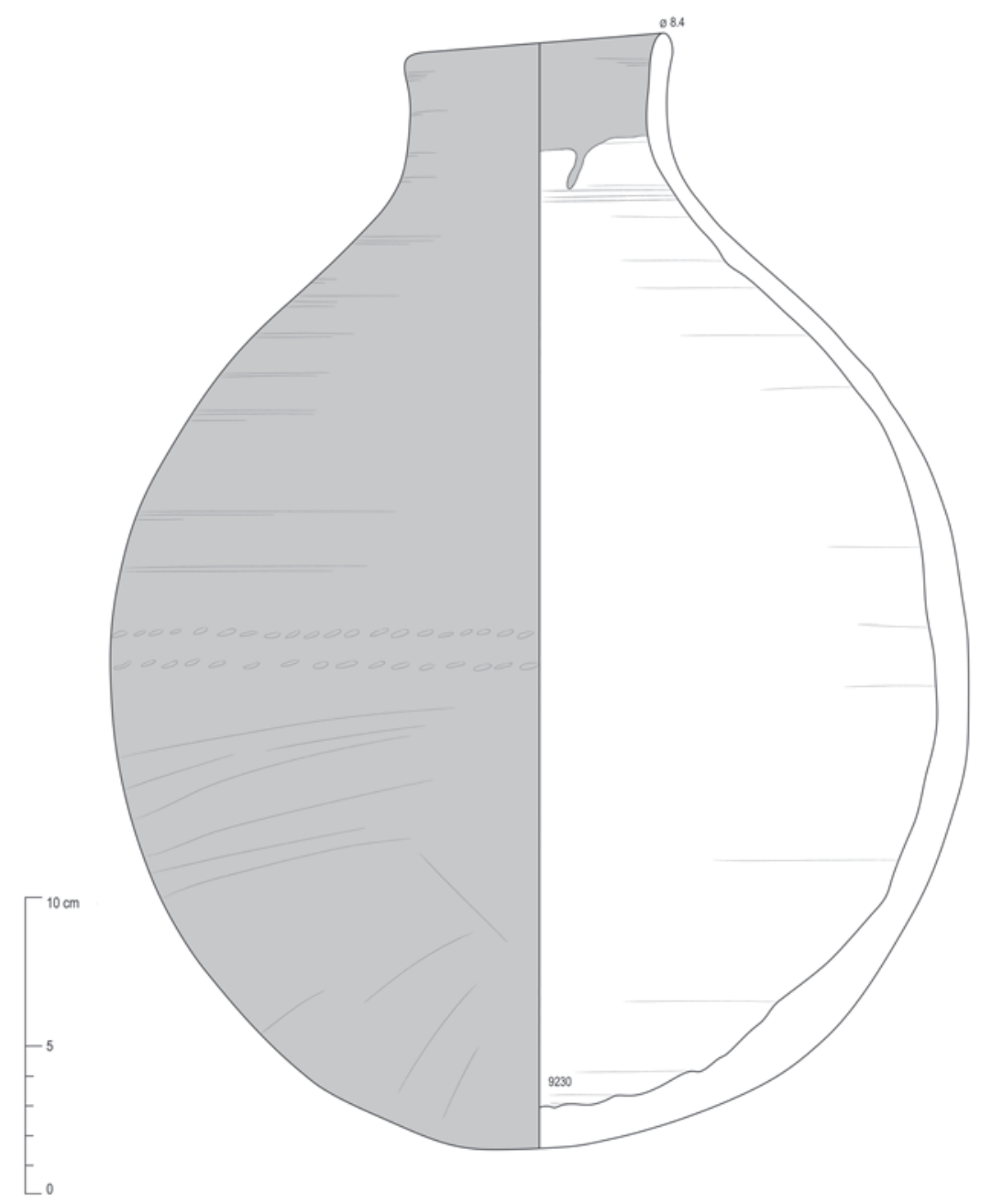

Fig. 16 Pottery from Tomb IV-L2, Middle Kingdom, drawn by B. Bader, inked by E. Schuster.

No. 9230, rd. $=8.3$, max.d. $=29.0$, ht. $=36.8$ 37.5 , Nile $\mathrm{C} 3$, surface dark red slipped on exterior and on rim on interior (10R5/6 red), natural surface colour 7.5YR6/4 light brown, coiled and turned, base scraped on exterior but very well smoothed.

\section{Marl A3 - Medium to large jar (Fig. 15.i)}

Although the fragments did not join there can be only little doubt that they belong to the same vessel. The best parallels belong to the early $12^{\text {th }}$ Dynasty. ${ }^{136}$

Tomb IV-L2-10, $\mathrm{rd} .=10.0$, pres.ht. $=9.8+17.9$, Marl A3, section uniform greenish yellowish, uncoated surface ( $7.5 \mathrm{Y} 8 / 2$ pale yellow), coiled and turned, base mould made, well smoothed. The section is shown in Fig. 1.h. The top of the rim shows three incised post-firing rim ticks.

136 SCHiestL/SEILER 2012, best available parallel is 422, no 1-3 from the early $12^{\text {th }}$ Dynasty.

\section{Nile B3 - New Kingdom BB jar (Fig. 15.j)}

Tomb IV-L2-9, bd.=9.0-9.5, pres.ht. $=22.0$, Nile B3, surface uncoated (7.5YR6/4 light brown), wheel turned, base string cut.

\subsection{4.) Tomb V (square 970-North1)}

Tomb V excavated in 2011 consisted of a vertical shaft of $7.0 \mathrm{~m}$ depth with one burial chamber in the west. It was very close to Tomb VI (see below). The fill of the shaft and the chamber were excavated in several layers (L2, L6, L7, L8, L9, L10 and L11). Notably one layer above those containing pottery (from L6 onwards) consisted of clean sand without any finds, ${ }^{137}$ which seems to indicate that after the last entering of the tomb no more disturbances took place. The absence of any New Kingdom pottery indicates that the location of the tomb

\footnotetext{
137 Seco Álvarez 2015, 119, plan 2 and 15; Seco Álvarez et al. in press, ms 9.
} 
had been forgotten by the time of the construction of the mortuary temple of Thutmosis III. Because a number of joins were made between L6 and L7, L8 and L9 as well as L7 and L9 in the sherd material, these four contexts are treated as one, with L10 and L11 shown separately. ${ }^{138}$ Several joins could be made over old breaks. Thus, the destruction of the original burial might have smashed the ceramic inventory of the tomb perhaps without removing too much of it. The ceramic material is quite homogeneous and belongs to the late Middle Kingdom ('late $12^{\text {th }}$ early $13^{\text {th }}$ Dynasty') with a possible extension into the Second Intermediate Period. Not a single body sherd of these layers belonged to the well-known New Kingdom corpus.

The small beakers (Nile B2), one in a niche of the chamber (Layer 11, no. 9396) and several unmistakeable fragments of the same type in Layer 9 with a rim diameter of about $7.0 \mathrm{~cm}$, suggest a later date in the Second Intermediate Period, perhaps even as late as the $17^{\text {th }}$ Dynasty. ${ }^{139}$ Comparison of our examples with earlier beakers ${ }^{140}$ from the late $12^{\text {th }}$ and $13^{\text {th }}$ Dynasty favours a date in the $17^{\text {th }}$ Dynasty, because the vessels walls are very straight. The beer jars without neck and shoulder appear in Seiler's work but are not included into her chronological synopsis. ${ }^{141}$ The Middle Kingdom Handbook gives this type a range in the $13^{\text {th }}$ Dynasty. The vessel type occurs exclusively in Thebes and is, thus, probably the locally developed 'beer' jar type. ${ }^{142}$

Also present in L6 and L7 were re-used sherd tools, such as scrapers and digging tools.

\section{Nile B1 - Hemispherical cup (Fig. 17.a)}

The shape of the cup is straight walled. Similar variants occur in the Theban necropolis in the Asasif and the Ramesseum. ${ }^{143}$

Tomb V-L7-14, rd.= 11.0?, pres.ht. $=5.0$, Nile B1, surface uncoated with red slipped rim band, coiled and turned, well smoothed.

\section{Nile B1 - Model dish (Fig. 17.b)}

This type has already been discussed, see above.

\footnotetext{
138 L10 only contained one better preserved beer jar base/ body no 9397. L11 represents a niche in the burial chamber that only contained no 9396 a small beaker jar. See below.

139 Cf. A. SeIler, Tradition \& Wandel, 86-87. Fig. 37. ZN02/116 dates this type to the $17^{\text {th }}$ Dynasty.

140 SChiestl/Seiler 2012, 542-543.

141 SeILER 2005, 10-71, Abb. 24 ZN 02/185, 127-130.
}

Tomb V-L8-1, rd.=11.0, pres.ht.=3.3, Nile B1, surface uncoated, wheelmade.

\section{Nile B2/C1 - Carinated cup (Fig. 17.c)}

This cup shape has an everted direct rim with concave contour. The carination is just preserved. ${ }^{144}$

Tomb V-L7-15/20, rd.= 11.0, pres.ht. $=5.5$, Nile $\mathrm{B} 2 / \mathrm{C} 1$, surface uncoated, coiled and turned, well smoothed.

\section{Nile B2 - Incurved cup/beaker (Fig. 17.d)}

The Nile clay fabric belongs unequivocally to Nile B2 of best Middle Kingdom tradition. ${ }^{145}$

Tomb V-L6-5, rd.= 7.0, pres.ht.=6.0, Nile B2, surface uncoated, coiled and turned, trimmed on the exterior.

\section{Nile B2 - Small beaker jars (Fig. 17.e-f)}

One rim and most of the body of one or more small beakers were found in the assemblage. A complete example of this type was found in L11 (see below). Rim sherds Tomb V-L9-12 and 16 also belong to this type and also show a rim diameter of $7.0 \mathrm{~cm}$. It is possible that the base/body fragment Tomb V-L9-10 represents the same vessel as one of the rims but none of them joined. This is the only type that may be of obvious later date than the late Middle Kingdom (cf. above).

Tomb V-L9-11, rd.=7.0, pres.ht.=4.3, Nile B2 with some limestone, surface red slipped on exterior and rim on interior, coiled and turned/wheelmade?

Tomb V-L9-10, pres.ht. $=15.6$, Nile B2 with some limestone, surface red slipped on exterior, coiled and turned/wheelmade? base trimmed on exterior.

\section{Nile B2 - Offering stand with bowl (Fig. 17.g)}

A regular occurrence in Middle Kingdom tomb assemblages, offering stands are known in several variations spanning the entire Middle Kingdom. ${ }^{146}$

Tomb V-L7-13, bd.=9.0, pres.ht.= 6.4, Nile B2 fine, surface uncoated, coiled and turned, on interior of base scraped in the lower part but smooth in the upper part.

\footnotetext{
142 Schiestl/Seiler 2012, 670-671; For B. Bader it is doubtful whether the fragment from Memphis belongs to this type.

143 Schiestl/Seiler 2012, 100-109, late $12^{\text {th }} /$ early $13^{\text {th }}$ Dynasty.

144 Schiestl/Seiler 2012, 236-242, occurs during the whole of the Middle Kingdom.

145 SChiestl/Seiler 2012, 336, no 3.

146 Schiestl/Seiler 2012, 350-366.
} 
a)
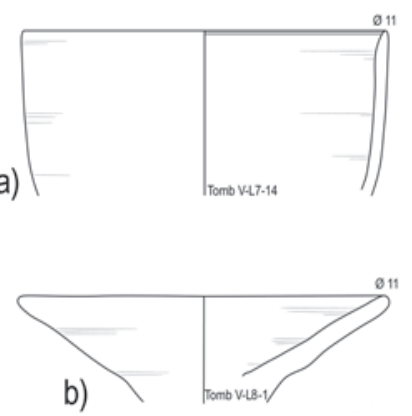

b)

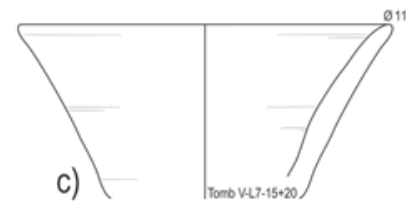

d)

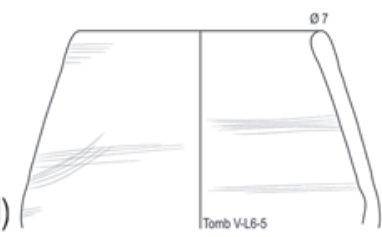

g)

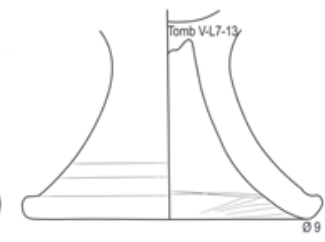

e)
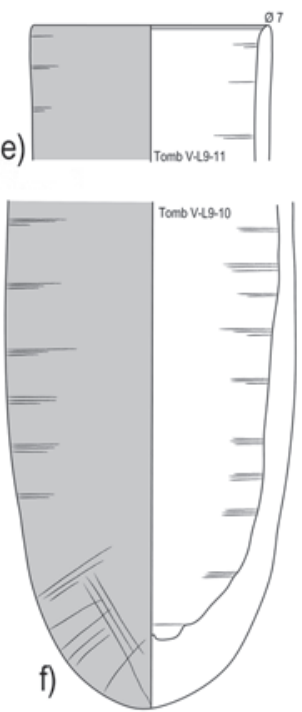
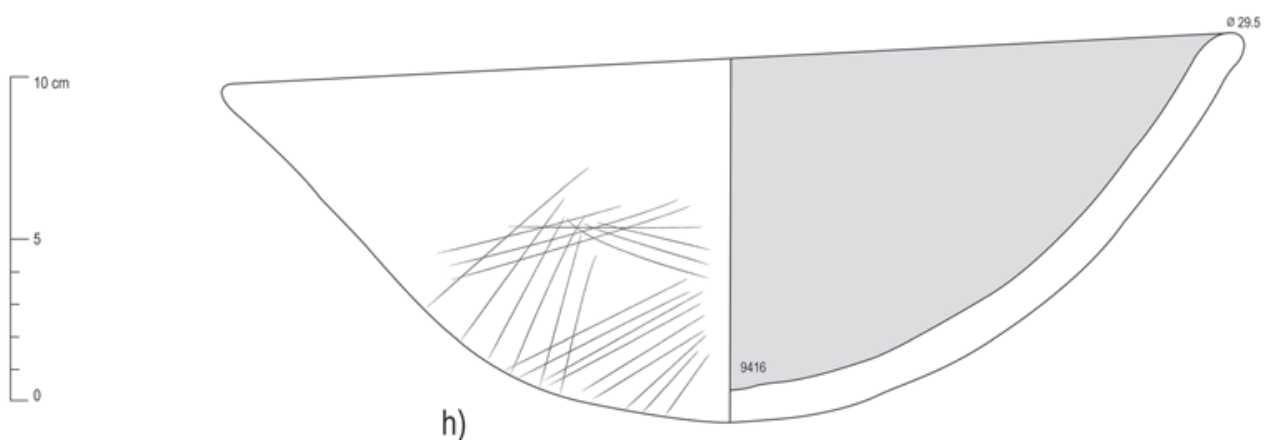

h)
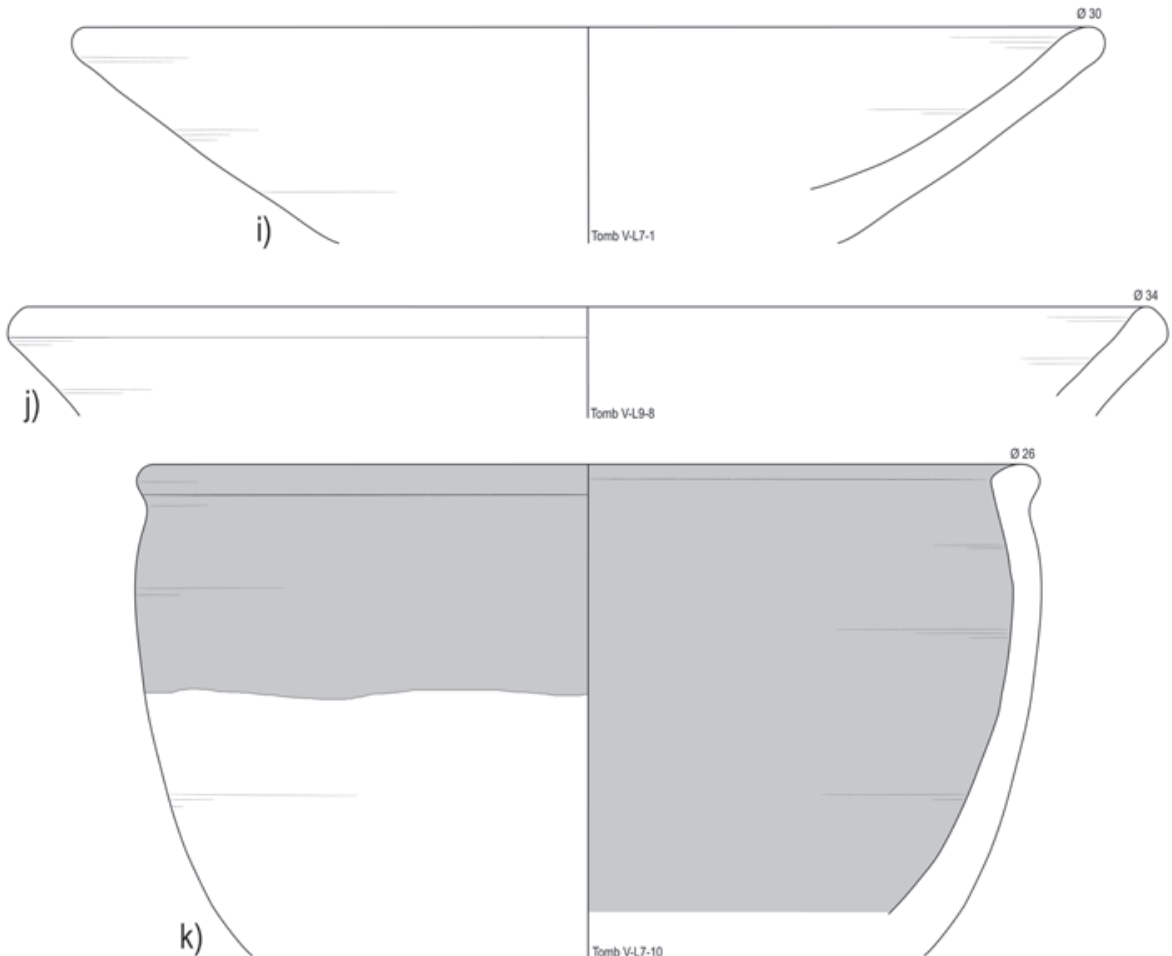

Fig. 17 Pottery from Tomb V-L6, 7, 8 and L9, late Middle Kingdom to Second Intermediate Period, drawn by B. Bader, inked by E. Schuster. 


\section{Nile C2 - Large dishes (Fig. 17.h-j)}

Such vessels appear in L6, L7, L8 and L9 and some of them seem to belong together although only one dish from L8 could be reconstructed to a full profile (no. 9416). The variations of the rim execution can be divided into simple direct (no. 9416, Tomb V-L7-1 and 4-9, Fig. 17.h-i), simple direct rims with rope impressions directly under the rim on the exterior (Tomb V-L6-2, Tomb V-L8-2,) and with trimmed tops of rims (Tomb V-L7-2-3, Tomb V-L9-7-8, Fig. 17.j). One round base of a dish occurred in Tomb V-L8/9-5 with rough scraping on the exterior. Rim diameters are in a range of $32.0-38.0 \mathrm{~cm}$. Parallels date to the $12^{\text {th }}$ and $13^{\text {th }}$ Dynasty. ${ }^{147}$

No. $9416,{ }^{148} \mathrm{rd} .=29.5$, ht. $=11.3$, Nile C2, section uniform light brown, surface red slipped on interior (10R5/4 red) uncoated exterior (7.5YR6/4 light brown), well smoothed, coiled and turned, base scraped with a tool, VI 261.

Tomb V-L7-1, rd.= 30.0, pres.ht.=6.6, Nile C2 with some limestone, surface uncoated, coiled and turned, interior smoothed, base scraped. (Tomb VI-L7-4-9 same vessel)

Tomb V-L9-8, rd.= 34.0, pres.ht.= 3.4, Nile C2, surface uncoated, coiled and turned.

\section{Nile C2 - Bowl (Fig. 17.k)}

Deeper bowls occur with folded lip. Already in L2 one such (smaller) fragment was found as the only diagnostic. Tomb V-L7-11-12 are the same as the example shown. Parallels do not occur in the Theban necropolis. ${ }^{149}$

Tomb V-L7-10, rd.=26.0?, pres.ht. $=14.8$, Nile C2 + some limestone, surface red slipped on interior, and $10 \mathrm{~cm}$ down the exterior, coiled and turned.

\section{Nile C2 - 'Beer' jars (Fig. 18.a-i)}

A large number of broken Middle Kingdom beer jar fragments made of Nile $\mathrm{C} 2$ and $\mathrm{C} 3$ either uncoated or with a red slip on the exterior was found in all four layers 6, 7, 8 and 9 amounting in total to $40.25 \mathrm{~kg}$ or 748 fragments. Prolonged trials to reconstruct more complete profiles were unsuccessful. L7 contained eight beer jar bases (Tomb V-L7-30-37, Fig. 18.a) all of which were made from Nile $\mathrm{C} 2$ without any other inclusions than

\footnotetext{
147 Schiestl/SeIler 2012, 322-323.

148 L8.

149 Schiestl/Seiler 2012, 322-325, 328-333.

150 SEILER 2005, Fig. 24, ZN 02/162.

151 Similar to SeILER 2005, Fig. 25, ZN 01/891 but even longer.
}

rough straw. Both red slipped on exterior and uncoated examples occurred, all were roughly scraped on the exterior. L9 included one more beer jar base (Tomb V-L9-9), Nile C2, uncoated and scraped on the exterior.

Tomb V-L7-33, pres.ht.= 12.0, Nile C2, surface red slipped on interior, coiled and turned, roughly scraped on exterior.

The co-occurrence of a funnel shaped beer jar rim with folded $\operatorname{lip}^{150}$ (Fig. 18.b), tubular necks with folded rims ${ }^{151}$ (and slight indentations on the interior) (Fig. 18.d, e), kettle rims ${ }^{152}$ (Fig. 18.c) and the neckless beer jar without pronounced shoulder ${ }^{153}$ can be observed in this context. One very small fragment representing just the top of the rim (Tomb V-L9-3 $\mathrm{rd} .=8.5$ ) could belong to a kettle rim or a tubular one.

It remains unclear if this assemblage thus represents a chronological range or a disturbed context depending on the validity of the argument that these rim types must follow each other. Whether there may be any overlap, cannot be estimated from the current find situation.

Tomb V-L9-2, rd.= $10.0(45 \%)$, pres.ht= 4.9, Nile C2, surface red slipped on exterior, coiled and turned.

Tomb V-L7-21, rd.=9, pres.ht. $=10.5$, Nile C2, surface red slipped on exterior and on interior on rim; same vessel is no 22 .

No. $9398,{ }^{154}, \mathrm{rd} .=9.5$, max.d. $=24.3$, pres.ht. $=$ 32.4 , Nile $\mathrm{C} 3$, section dark grey core close to inner edge of section with light brown and reddish oxidation zones, see Fig. 1.e, surface thick dark red slipped on exterior (10R5/6 red), natural colour 2,5YR6/6 red, vessel was made in two parts, coiled and turned, base probably scraped, the neck was smoke stained in places.

Tomb V-L6/7-4, rd.= 11.0, pres.ht.= 5.2, Nile $\mathrm{C} 3$, surface red slipped on exterior and on rim interior, coiled and turned.

Tomb V-L7-23, rd.= 8.0, pres.ht.= 11.5, Nile C2, surface red slipped on exterior, coiled and turned, nos 24-25 belong to same vessel, very similar no 26 with rim diameter 7.5 and nos 27-28 (rim diameter 7.0).

Tomb V-L9-1, rd.= 8.0, pres.ht.=4.6, NIle C2, surface uncoated, coiled and turned.

\footnotetext{
152 Cf. SeILER 2005, Fig. 28, ZN 01/400.

153 Cf. Seiler 2005, Fig. 24, ZN 02/185; Schiestl/Seiler 2012, 670-671, type II.H.4.1, $13^{\text {th }}$ Dynasty.

154 Joined from sherds of L7 and L8.
} 

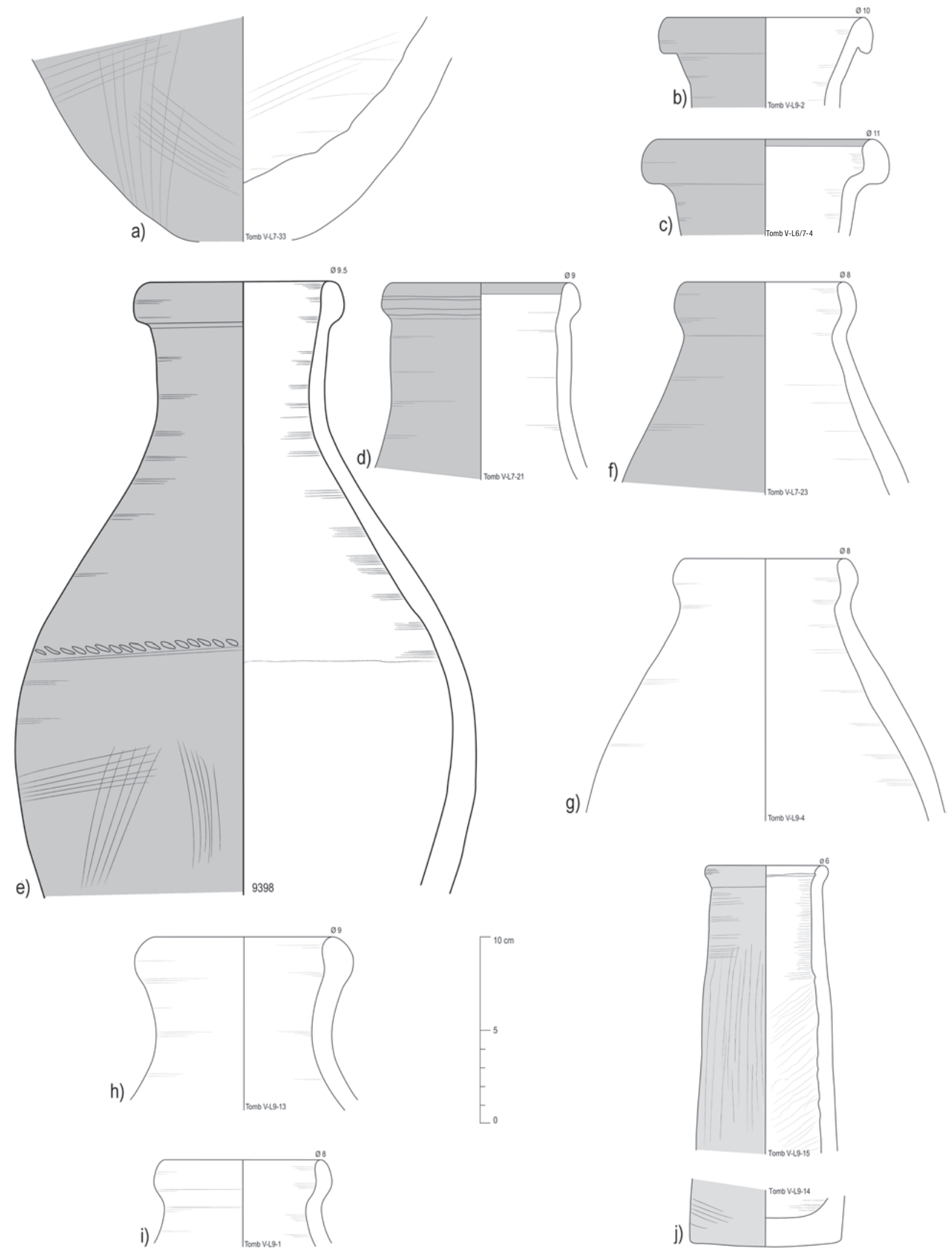

Fig. 18 Pottery from Tomb V-L6, 7, 8 and L9, late Middle Kingdom to Second Intermediate Period, drawn by B. Bader, inked by E. Schuster. 


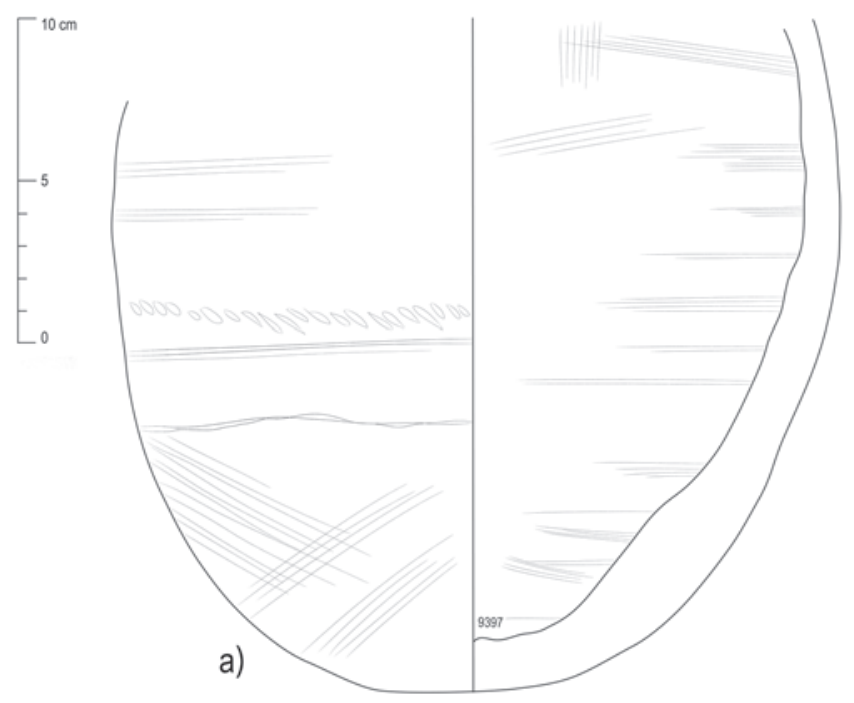

b)

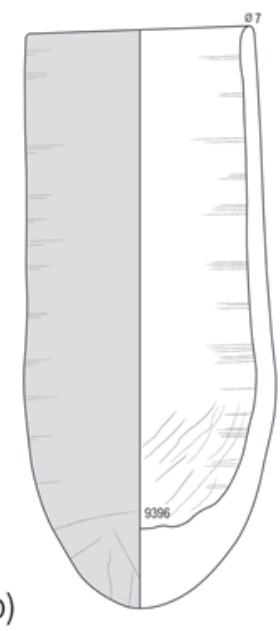

Fig. 19 a) Pottery from Tomb V-L10, late Middle Kingdom to Second Intermediate Period. b) Pottery from Tomb V-L11, late Second Intermediate Period to New Kingdom, drawn by B. Bader, inked by E. Schuster.

Tomb V-L9-4, rd.=8, pres.ht. $=14.1$, Nile C2, surface uncoated, coiled and turned.

Tomb V-L9-13, rd.=9.0, pres.ht. $=9.3$, Nile C2, surface uncoated, coiled and turned, exterior covered with white mortar (gypsum/lime?).

\section{Marl A2 - Conical vessel (Fig. 18.j)}

This is another example ${ }^{155}$ of such a vessel that was already discussed under Tomb IV (see p. 189). Due to the fact that no other late material was present in Tomb V, it seems very likely that the vessel type is contemporary with the late Middle Kingdom or the Second Intermediate Period.

Tomb V-L9-14-15 156 , rd.=6.0, bd. $=8.1$, pres. ht. $=3.7+15.4$, Marl A2, see Fig. 1.g for the section, surface with thick white scum, coiled and turned, vertically trimmed on exterior, lower part squeezed together (folds on interior), base hand formed.

The only pottery found in Tomb V-L10 is the base of an ovoid beer jar of the late Middle Kingdom to the early Second Intermediate Period. ${ }^{157}$

\section{Nile C2 - 'Beer' jar (Fig. 19.a)}

No. 9397, max.d.= 22.4, pres.ht. $=20.8$, Nile C2, section, dark grey core inside, light brown oxida-

\footnotetext{
155 Seco Álvarez et al. in press, fig. 24.c.

156 Non-joining sherds.

157 Schiestl/Seiler 2012, 650-676.

158 Seco Álvarez et al. in press, ms 9.
}

tion zone outside, surface uncoated (5YR7/4 pink), coiled and turned, held together by rope during the drying process, base made by hand, subsequently scraped on the outside, join line indicated manufacture in at least two parts. The base was filled with the same material as the tomb shaft: limestone chips: small and larger, from a few centimetres to chips of $4-5 \mathrm{~cm}$ thickness and $8-9 \mathrm{~cm}$ length. Other finds were rodent or lizard bones, not articulated, one very small white ring bead, one "miniature silex sickle blade". Also in the fill were blank lumps of sealing clay. Adhering to the vessel walls below the secondary fill, there were remains of a pure mud fill. The mud was dark, compacted and without any inclusions.

From the niche Tomb V-L11 one complete profile of a single small beaker jar is derived (Fig. 19.b). ${ }^{158}$ This type has been discussed above (p. 194).

No. 9396, rd.=7.0, max.d.=7.7, ht. $=17.7$, Nile $\mathrm{B} 2 / \mathrm{C} 1$, section dark grey core, red and light brown oxidation zones, surface red slipped on exterior (10R6/6 light red), natural colour 7.5YR6/4 light brown, coiled and turned, base made from lump on the wheel and subsequently scraped outside, the interior shows clearly folds from squeezing together and slightly turning the vessel, ${ }^{159}$ the joins could be

159 This is a very clear criterion to distinguish red slipped, burnished body sherds of such beakers, which also occur, from dipper juglets that are always made on a wheel with quite deep grooves on the interior. 
made over old breaks, which means the pot was broken during deposition or soon afterwards.

\subsection{5.) Tomb VI (square $970-$ North1) $)^{160}$}

Tomb VI also excavated in 2011 is located just a short distance to the east of Tomb $\mathrm{V}$ in the same square with a very similar architecture of a vertical shaft of $7.0 \mathrm{~m}$ depth and a funerary chamber. ${ }^{161}$ The rectangular shaft mouths of the two tombs are parallel to each other suggesting a similar date for their construction. The layers belonging to the shaft fill from top to bottom of the shaft are L3, ${ }^{162}$ L12 and L13. The entrance to the burial chamber was located at the west of the shaft and in front of the mud brick wall closing the burial chamber a pottery deposit was found, L14. Interestingly this wall was built on top of the pottery deposit L15, mainly consisting of a large dish, which indicates at least two use-phases of the burial chamber. L 16 and L17 represent fill at the entrance of the burial chamber. ${ }^{163}$ Joins between vessels of L15 and L17 prove the contemporaneity of the material and that it was scattered later.

The ceramic material consists of a mixture of Middle and New Kingdom ceramic material (mid $18^{\text {th }}$ Dynasty, often only in the form of non-diagnostic fragments), where the New Kingdom pottery becomes less prominent the further down the shaft was excavated. Several joins between layers also exemplified the mixed nature of the shaft and burial chamber fill. Multiple joins between vessels were made between L13 and L14, L14 and L15, L15 and L17, which shows that they were at least accessed and moved around at the same time. Thus these contexts will be presented together (see below). Most of the pottery belongs to the corpus of well-known Middle Kingdom types of late $12^{\text {th }}$ or $13^{\text {th }}$ Dynasty date.

In addition to the diagnostic and recorded material the non-diagnostic pottery consisted mainly of body fragments of heavy Middle Kingdom 'beer jars (in total of all Layers $46.0 \mathrm{~kg}$, both red slipped on exterior and uncoated occurred) with $0.5 \mathrm{~kg}$ of fragments of open Middle Kingdom vessels of Nile C2 and small amounts of hemi-

160 Seco Álvarez 2015, plan 2, fig. 15.

161 Seco Álvarez et al. in press, ms 4.

162 This is the context on the very top of the shaft, a surface layer. Already in this surface layer some ceramic material of clear Middle Kingdom date (hemispherical cups, beer jar kettle rim, Marl A3 jar) was found. spherical cup fragments. Only singular New Kingdom fragments like ring pattern burnished open vessels or $0.25 \mathrm{~kg}$ of New Kingdom closed vessels were found in these contexts. The conclusion one might draw from a comparison of tombs V and VI reveals that they probably were built at roughly the same time, but that their post depositional history differs quite distinctively, after the burials and after they had been robbed.

The surface layer above the tomb shaft, 970-North1-L3 yielded two interesting fragments, which are presented for completeness' sake of the site corpus.

\section{Marl A3 - Globular jar (Fig. 20.a)}

This globular jar belongs to the corpus of the Middle Kingdom. ${ }^{164}$

970-N1-L3-10, pres.ht.= 11.6, Marl A3, surface uncoated, coiled and turned, very well smoothed.

\section{Nile B2 - Medium jar (Fig. 20.b)}

This vessel type is by parallels dated to the New Kingdom. ${ }^{165}$

970-N1-L3-4, rd. $=6.0$, pres.ht. $=9.8$, Nile B2, fine but hard fired variant, surface red slipped on exterior to just below neck and rim on interior, wheelmade, with folding on interior suggesting squeezing into shape.

The uppermost fill of the shaft, Tomb VI-L12, contained the Middle and New Kingdom mix alluded to above. The Middle Kingdom components consists of Nile B1 hemispherical cups of open shape with a rim diameter of $11.0-12.0 \mathrm{~cm}$ and a thin red rim band, several straight beer jar rims made from Nile $\mathrm{C} 2$ with a slight indentation on the interior, and 36 diagnostic fragments of small dishes made from Nile B1 and Nile B2/C1 (cf. above, Tomb III).

\section{Nile B1 - Model bowl (Fig. 20.c)}

This model vessel type of restricted shape does not occur in the Theban necropolis. ${ }^{16}$

Tomb VI-L12-13, rd.= 5.0, pres.ht. $=3.6$, Nile B1, surface uncoated, coiled and turned.

\footnotetext{
163 See Seco Álvarez 2015, plan 2; Seco Álvarez et al. in press, ms 4.

164 Schiestl/Seiler 2012, 420-422, 444-446.

165 Aston 2003, 141, fig. 1a, second and third row from top.

166 Schiestr/Seiler 2012, 933, but in Tomb VI made from Nile B1.
} 
Nile C2 - 'Beer' jar (Fig. 20.d)

This beer jar rim belongs very likely to Seiler's type DAN $2 .{ }^{167}$

Tomb VI-L12-3, $\mathrm{rd} .=10$, pres.ht. $=6.3$, Nile $\mathrm{C} 2$, surface red slipped on the exterior, coiled and turned.

The pottery from the layers Tomb VI-L13, L14, L15 and L17 is presented in the following in a combined manner due to the multiple joins between them.

\section{Nile B1 - Hemispherical cups (Fig. 20.e-f)}

Several fragments of straight walled cups were found. They showed thin red slipped bands and a rim diameter of $10.0-11.0 \mathrm{~cm} .{ }^{168}$

Tomb VI-L17-5, rd.= 11.0?, pres.ht.= 3.5, Nile B1, surface uncoated with red slipped rim band, coiled and turned, interior smooth.

Tomb VI-L17-7, $\mathrm{rd} .=10.0$, pres.ht. $=2.9$, Nile B1, surface uncoated with red slipped rim band, coiled and turned, interior smooth.

\section{Nile B1 and B2 - Conical cups with sharp ridge on interior (Fig. 20. g-h)}

These cups are characterised by their wide open shape with direct or thickened rims that may be trimmed with a tool from the top. The bases are string cut, whilst on the interior close to the base a separate area is often defined by a sharp ridge. ${ }^{169} \mathrm{It}$ is possible that this 'cup in a cup' was used for liquid offerings or libations. The same type made from a slightly coarser fabric was found in Layer 16. Another feature in such vessels was the seemingly intentional 'spout' broken into the rim as if to ease pouring liquids from it. This feature was also noticed in the carinated bowls of the early Middle Kingdom (see above, Fig. 3.a, 4.e, 20.h) as well as at other sites. ${ }^{170}$ Several fragments of such cups were found in Layers 13 and 14 (Tomb VI-L13/14-27, bd.=4.6), which have no parallels from the Theban necropolis.

Tomb VI-L13/14-7, $\mathrm{rd} .=9.0$, bd. $=4.0$, ht. $=5.6$, Nile B1, surface uncoated, coiled and turned, base string cut.

\footnotetext{
167 SEILER 2005, 70-73.

168 Schiestl/Seiler $2012,100-107$, late $12^{\text {th }} /$ early $13^{\text {th }}$ Dynasty.

169 Cf. Schiestl/SeIler 2012, 126-127, unfortunately it is not stated whether these bases were string cut; dating late $12^{\text {th }}$ to $13^{\text {th }}$ Dynasty.

170 E.g. Ehnasya el-Medina.
}

No. $8772(\mathrm{~L} 14), \mathrm{rd} .=12.0$, bd. $=4.5$, ht. $=5.5$, Nile B2, section not visible, uncoated (7.5YR7/3 pink), coiled and turned on device, base string cut, top of rim trimmed with a tool from top.

\section{Nile B1 - Model jar (Fig. 20.i)}

This type of model jar may represent a canopic jar shape or nmst although this cannot be certain. This type is not otherwise known from the Theban necropolis. ${ }^{171}$

Tomb VI-L13/14-6, rd.=2.5, pres.ht. $=6.1$, Nile B1, section uniform light brown, surface uncoated but covered with white plaster, coiled and turned, base string cut.

\section{Nile B2/C1 - Model/Small dishes (Fig. 20.j-k)}

Open vessels of small size occur throughout the Middle Kingdom. ${ }^{172}$

No. 20257 (L14), rd.=8.2, bd.=4.4, ht. $=2.5$, Nile B2/C1, section uniform brown, surface uncoated (5YR5/4 reddish brown), completely covered with white lime plaster, ${ }^{173}$ made on slow wheel (W1), base string cut.

No. 20258 (L14), rd.= 7.8, bd.=3.6, ht. $=2.4$, Nile $\mathrm{B} 2 / \mathrm{C} 1$, section uniform brown, surface uncoated (7.5YR5/4 brown), covered with lime plaster, ${ }^{174}$ made on slow wheel (W1), base string cut.

\section{Nile B2 - Model jars (Fig.20.I-n)}

Closed vessels of small size can also be found in the late $12^{\text {th }}$ and early $13^{\text {th }}$ Dynasty. ${ }^{175}$

No. $20260^{176}(\mathrm{~L} 14), \mathrm{rd} .=3.8$, max.d. $=5.3$, bd. $=$ 3.3 , ht. $=7.0$, Nile B2, section uniform light brown, surface uncoated (7.5YR6/4 light brown), made on slow wheel (W1), base string cut.

No. 8604 (L17), rd.=3.5, $\max . d .=8.5$, bd. $=3.7-$ 3.8 , ht. $=8.0$, Nile B2 fine, section not visible, surface uncoated (7.5YR7/4 pink), coiled and turned on device/wheel (W1), base string cut, uneven, intact, weight $102 \mathrm{~g}$.

No. 8605 (L17), rd.=3.0-3.4, max.d.=5.6, bd.= 3.8 , ht. $=8.1$, Nile B2 fine, section yellowish red, surface uncoated (7.5YR7/4 pink), coiled and turned on device/wheel (W1), base string cut, intact, weight $111 \mathrm{~g}$.

\footnotetext{
171 Cf. Schiestl/SeILer 2012, 963, similar to type IV.2.C2.a.7, but made from Nile B1.

172 Cf. Schiestl/Seiler 2012, 846-848, esp.847, no 5, 7.

173 Strong reaction to lemon juice.

174 Strong reaction to lemon juice.

175 Schiestl/Seiler 2012, 980-981.

176 Drawn by D. Penzer.
} 

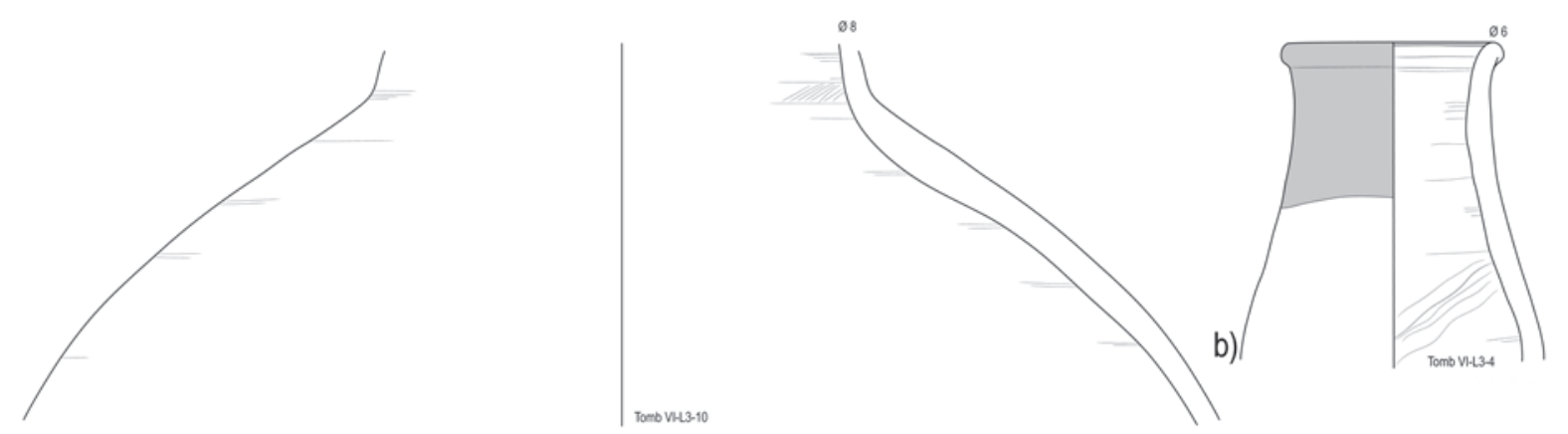

a)
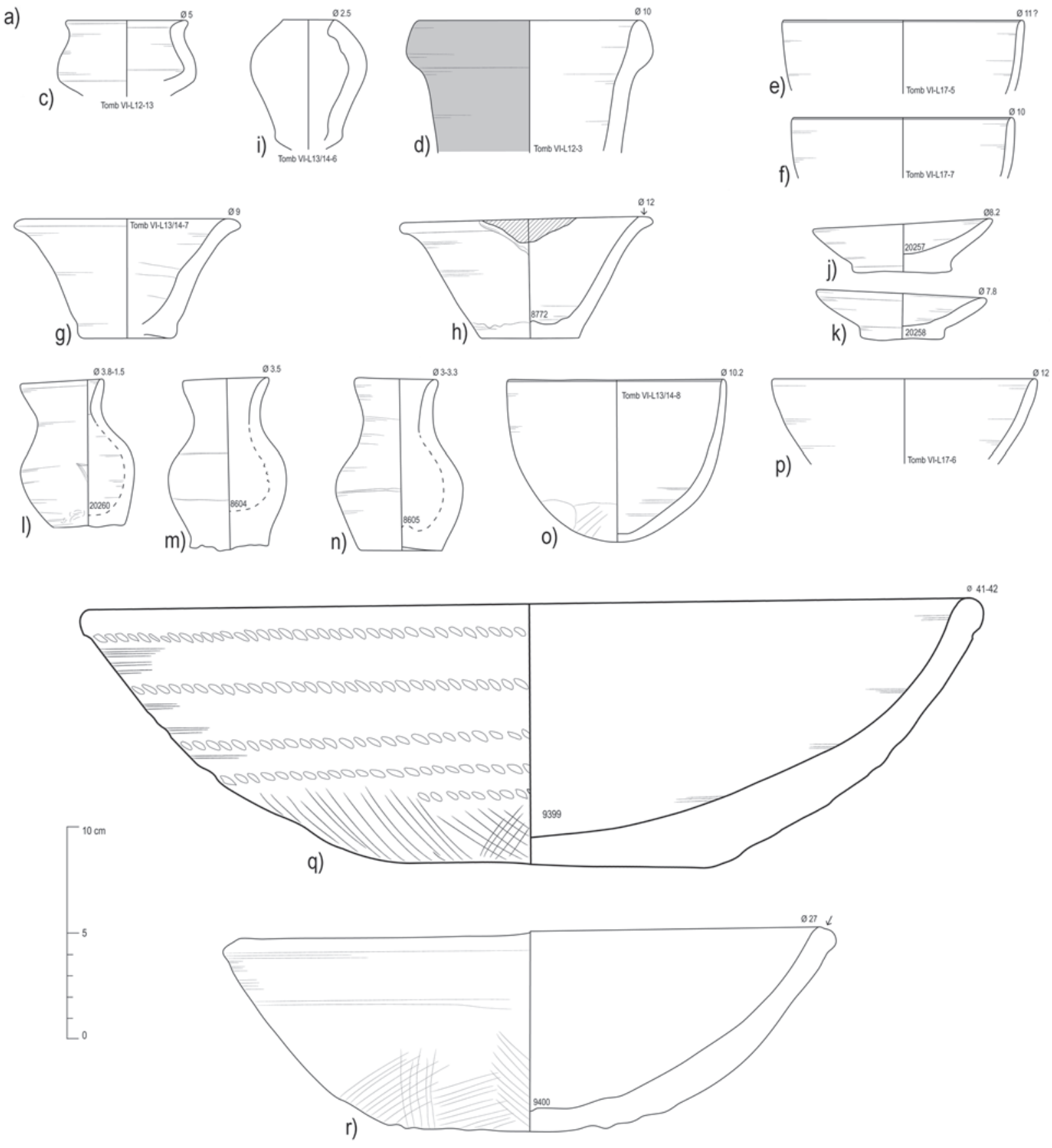

Fig. 20 Pottery from Tomb VI-L3, L12-17, Middle and New Kingdom, drawn by B. Bader, inked by E. Schuster. 
a)
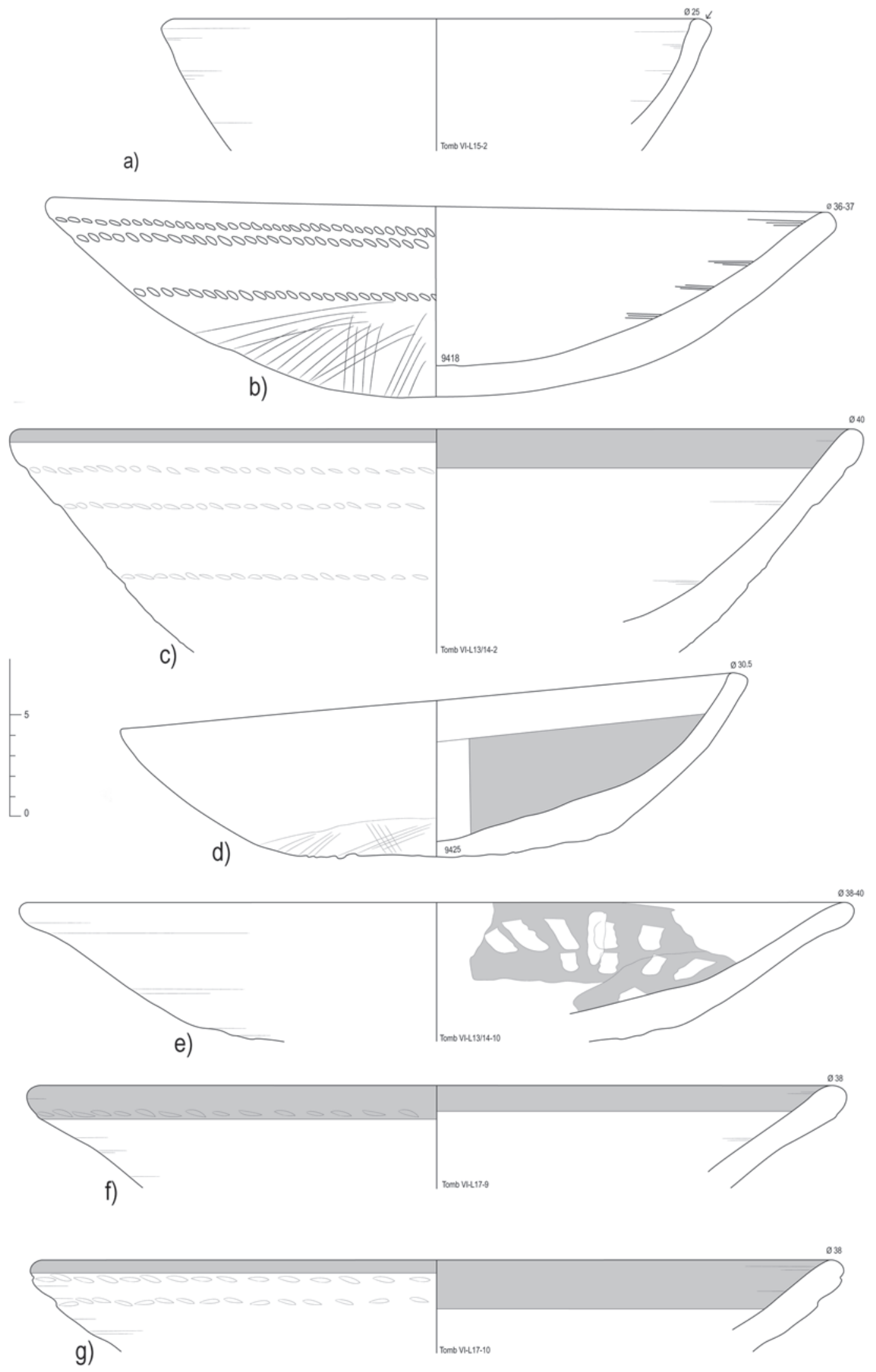

Fig. 21 Pottery from Tomb VI-L12-17, late Middle Kingdom, drawn by B. Bader, inked by E. Schuster. 
Nile B2 - Hemispherical cups (Fig. 20.0-p)

One profile of such a cup could be reconstructed and it represents a slightly deeper vessel with a rim diameter of ca. $10.2 \mathrm{~cm}$, whilst another fragment without red rim band shows an open form, thick walls and hints at an earlier date. ${ }^{177}$

Tomb VI-L13/14-8, rd. $=10.2$, max.d. $=10.4$, ht.= 7.7, Nile B2 fine variant, section uniform light brown, surface uncoated with thin red rim band, coiled and turned, smooth interior, base trimmed with tool, vessel index 135.1.

Tomb VI-L17-6, rd. $=12.0$, pres.ht. $=4.0$, Nile B2, surface uncoated, coiled and turned, smooth on interior, relatively thick vessel walls.

\section{Nile C2 - Large Dishes and bowls (Fig. 20.q-r, 21.a-g)}

Large Nile C2 dishes and bowls belong to almost every Middle Kingdom burial assemblage. ${ }^{178}$ In this instance a good selection of shallower and deeper dishes and bowls came to light, which most probably belonged to the original assemblage. Especially noteworthy are two dishes of Nile C2 with white painted decoration on the interior over a red slip: one with a white cross (no. 9425, Fig. 21.d ${ }^{179}$ and one with radial white finger strokes (Tomb VI-L13/14-10, Fig. 21.e) on a red slip. Two further uncoated base fragments were found (Tomb VI-L15-3 (100\% preserved), Tomb VI-L17$20(25 \%$ preserved)). The rims may be trimmed or direct, sometimes even in one vessel. Slightly incurved varieties do not exist in the Middle Kingdom Handbook.

No. 9399, (L15 joins L17), rd.=41.0-42.0, bd.= $15.0-16.0, \mathrm{ht} .=11.6-13.2$, Nile $\mathrm{C} 2$, section uniform light brown, surface uncoated (7.5YR6/4 light brown), coiled and turned, rope impressions on the outside, base scraped with a tool, joins over old breaks.

No. 9400 (L15), rd.= 27.0, ht. $=9.5-10.5$, Nile $\mathrm{C} 2$, section uniform light brown, surface uncoated (7.5YR6/4 light brown), coiled and turned, base roughly scraped on exterior, top of rim trimmed with a tool at an angle.

Tomb VI-L15-2, rd.= 25.0, pres.ht. $=6.4$, Nile $\mathrm{C} 2$, surface uncoated, coiled and turned, top of rim trimmed with tool obliquely. Sherd 1 and 5

177 Schiestl/Seiler 2012, 88-107.

178 Schiestl/Seiler 2012, 192-194, 198-201, 212-213, 150151. very similar. Same type as no. Tomb VI-L17-14 to 19.

No. 9418 (L17), rd.= 36.0-37.0, ht. $=8.3-10.5$, Nile $\mathrm{C} 2$, section thin dark brown core, light brown oxidation zones, surface uncoated (10R7/4 very pale brown), coiled and turned, smoothed afterwards on wheel, base roughly scraped with a tool, three rows of rope marks on exterior, only one small sherd missing: weight $2.75 \mathrm{~kg}$.

Tomb VI-L14-2, rd. $=40.0$, pres.ht. $=10.9$, Nile $\mathrm{C} 2$, surface uncoated with red rim band $(2.0 \mathrm{~cm}$ on interior, $0.8 \mathrm{~cm}$ on exterior), coiled and turned, three rows of horizontal rope marks.

No. 9425 (L13 joins L14), rd.= 30.5, max.d.= 30.8 , ht. $=8.1-7.0$, Nile $\mathrm{C} 2$, section thin dark grey core light brown oxidation zones, surface uncoated exterior (10YR7/3 very pale brown) and red slip on interior (5YR6/4 light reddish brown), white cross painted on interior (10YR8/1 white), combination technique of coiling and turning, base coiled and scraped on exterior.

Tomb VI-L13/14-10, rd.= ca 38.0-40.0, pres. ht. $=6.8$, Nile $C 2$, surface red slipped on interior with radial white finger decoration on the interior, exterior uncoated, coiled and turned, base roughly scraped.

Tomb VI-L17-9 (and11), rd.= ca 38.0, pres.ht.= 5.1, Nile C2, surface uncoated with red rim band, coiled and turned, one row of rope impressions on exterior.

Tomb VI-L17-10 (and 12, 13), rd.= ca 38.0, pres. $h t .=4.4$, Nile $\mathrm{C} 2$, surface uncoated with red rim band, coiled and turned, two rows of rope impressions on exterior.

Nile C2/3 - 'Beer' jars (Fig. 22.a-g, 23.a)

Several fragmentary beer jars of Middle Kingdom date are derived from Tomb VI. At least five bases and base fragments were represented in this assemblage (Fig. 22.a, g, 23.a). Their bodies were ovoid or ellipsoid, all rim fragments show kettle rims (Fig. 22.b-f). These fragments can be equated with Seiler's type DAN 3. ${ }^{180}$ A similar vessel occurs in the Ramesseum. ${ }^{181}$

No. 8767, (L13 and L14), (body only), max.d.= 27.5 , pres.ht. $=40.2$, Nile $\mathrm{C} 3$, section dark grey to black core (interior) with thin pink to red oxida-

\footnotetext{
179 Cf. SeILer 2005, Fig. 20, ZN 01/588, $13^{\text {th }}$ Dynasty. But this example has a folded rim.

180 SEILER 2005, 70-75.

181 LOYRETTE et al. 1993, fig. 4.n.
} 


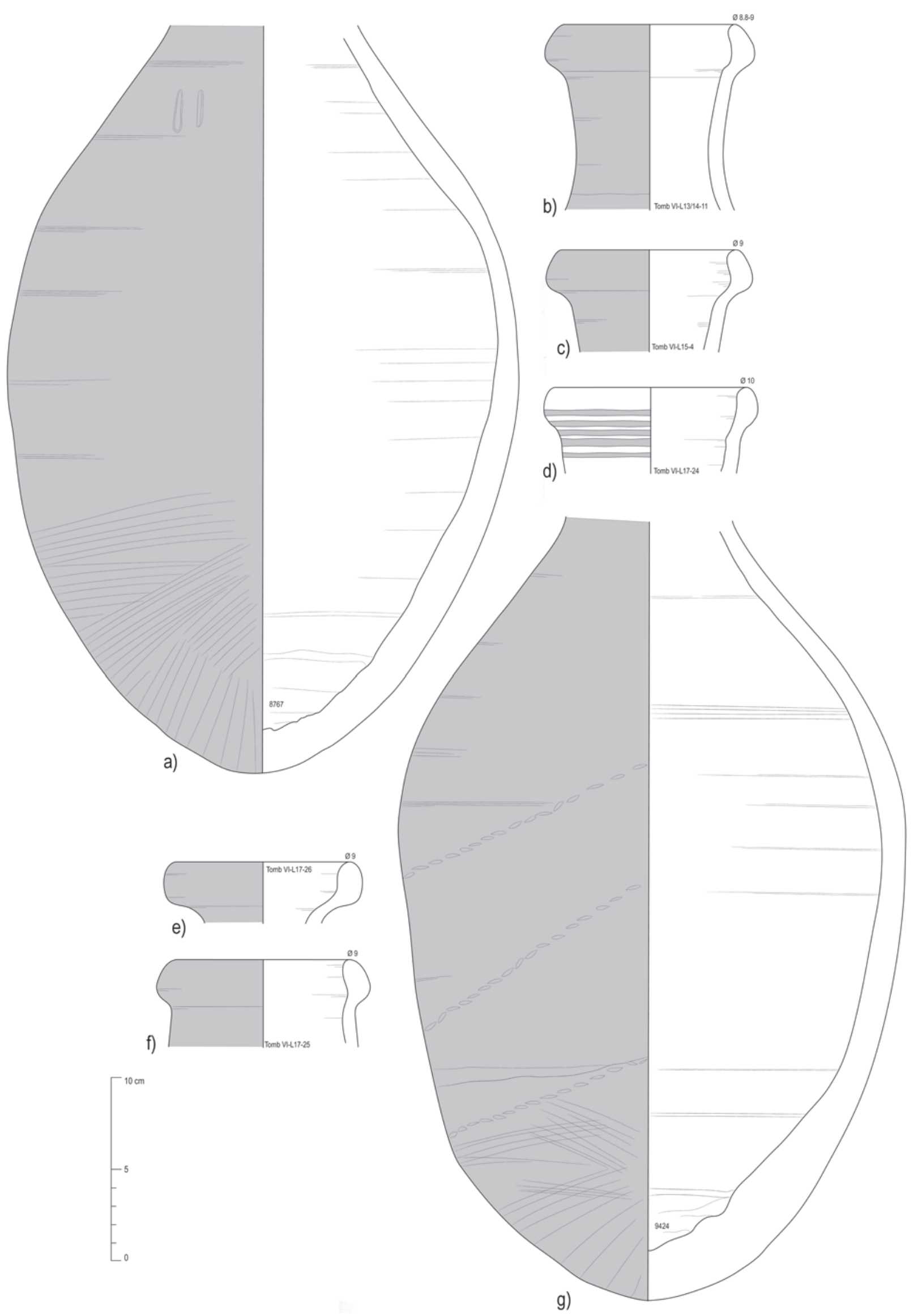

Fig. 22 Pottery from Tomb VI-L12-17, late Middle Kingdom, drawn by B. Bader, inked by E. Schuster. 
tion zones, surface traces of red slip on exterior, coiled and turned on device/wheel, base coiled and subsequently scraped, body visibly joined on in second step. Two vertical incisions were made close to the neck, pre-firing.

No. 9424 (L14), pres.ht.= 42.5, Nile C2, section dark grey core, red and brown oxidation zones, surface red slip on exterior (10R4-5/6 red) down to the base, base itself remained uncoated, natural colour (7.5YR7/4 pink), coiled and turned, base roughly scraped on exterior, vessel made in three parts, joining lines in lower third and just below the neck.

No. 9417 (L17), max.d.= 27.2, pres.ht.= 25.9, Nile C2, section greyish core, red and light brown oxidation zones, surface uncoated (10R6/4 pale red), red splash of colour on exterior (7.5R $7 / 4$ red), coiled and turned on wheel, base scraped with a tool, rope marks, only lower part of body preserved, one kiln stain at height of max.d.

\section{Beer jar kettle rims (Fig. 22.b-f)}

Tomb VI-L13/14-11, rd.= 8.8-9.0, pres.ht. $=10.2$, Nile C2, surface red slipped on exterior and interior on rim, coiled and turned.

Tomb VI-L15-4, $\mathrm{rd} .=9.0$, pres.ht. $=5.5$, Nile C2, surface red slipped on exterior, coiled and turned.

Tomb VI-L17-24, rd. $=10.0$, pres.ht. $=4.6$, Nile $\mathrm{C} 2$, surface traces of red slip on exterior, coiled and turned.

Tomb VI-L17-26, $\mathrm{rd} .=9.0$, pres.ht. $=3.4$, Nile $\mathrm{C} 2$, surface dark red slipped on exterior, coiled and turned.

Tomb VI-L17-25, rd. $=9.0$, pres.ht. $=4.8$, Nile $\mathrm{C} 2$, surface dark red slipped on exterior, coiled and turned.

\section{Marl A2 - Conical vessels (not shown)}

The body/base fragment as well as one rim fragment of such vessels also appeared in L13 of the tomb. They were made by hand and vertically scraped with the top of the vessel turned on a turntable.

\section{Tomb VI-L16}

The numerous and highly fragmented pottery from this layer was described as lying below one

182 Seco Álvarez et al. in press, ms 4.

183 Schiestr/Seiler 2012, 124-125.

184 Aston 1994, 145.157, 146.159 and 147.161. They are similar but not the same; BietaK 1991, 34, no 1698, calcite. The item resembles the shape quite well, late Middle Kingdom date. of the large dishes from L15 (no. 9400, 9399). ${ }^{182}$ The vessels encountered were small and finely executed and seem to represent at least part of the assemblage of the original burial, especially those that look like kohl jars.

\section{Nile B2 - Cups/Bowls with out-turned rims (Fig. 23.b-d)}

In contrast to the conical cups discussed before (Fig. 20.g-h) the current cups exhibit a tapering direct rim and a distinct sharp edged 'cup' in the middle of the interior. The base is string cut and the quality of the production is high. There are no exact parallels in the Theban necropolis, although there are similar vessels without the 'cup'. ${ }^{183}$

No. $8773, \mathrm{rd} .=11.3-11.5, \mathrm{bd} .=4.6-4.8, \mathrm{ht} .=$ 6.3-6.6, Nile B3, section not visible, surface uncoated (7.5YR7/4 pink), coiled and turned on device/wheel (W1), base string cut, intact, weight $183 \mathrm{~g}$.

No. 8774, rd. $=11.8$, bd. $=4.6-5.0$, ht. $=6.3$, Nile B2 fine, section red core with reddish brown oxidation zones, surface uncoated (5YR6/4 light reddish brown), made on slow wheel (W1), base string cut.

No. 8771 , rd. $=11.8$, bd. $=4.5$, ht. $=6.6$, Nile B3, section not visible, surface uncoated (7.5YR7/4 pink), coiled and turned on device/wheel (W1), base string cut, intact, weight $178 \mathrm{~g}$.

\section{Nile B2 - Kohl jars (Fig. 23.e-f)}

These remarkable vessels resemble in shape very closely stone vessels that are usually produced from 'calcite/alabaster/travertine'. ${ }^{184}$ The replacement of stone with clay, if indeed it was one, is not very well known in the southern part of Egypt. In the north, at Tell el-Daba, such shapes occur but so far only in the Second Intermediate Period. ${ }^{185}$

No. $20261,{ }^{186} \mathrm{rd} .=5.0$, max.d. $=5.8$, bd. $=3.4$, ht. $=6.3$, Nile B2, section uniform light brown, surface uncoated (10YR7/3 very pale brown), wheelmade (W1), base string cut.

No. $20259,{ }^{187} \mathrm{rd} .=5.0$, max.d. $=5.6$, bd. $=4.0$, ht. $=6.3$, Nile B2, section not visible, surface uncoated (7.5R6/4 light brown), wheelmade (W1), base string cut.

\footnotetext{
185 Cf. Bietak 1991, for example 176-178, Fig. 137.9, Phase E/1, Second Intermediate Period, black burnished surface.

186 Drawn by D. Penzer.

187 Drawn by D. Penzer.
} 

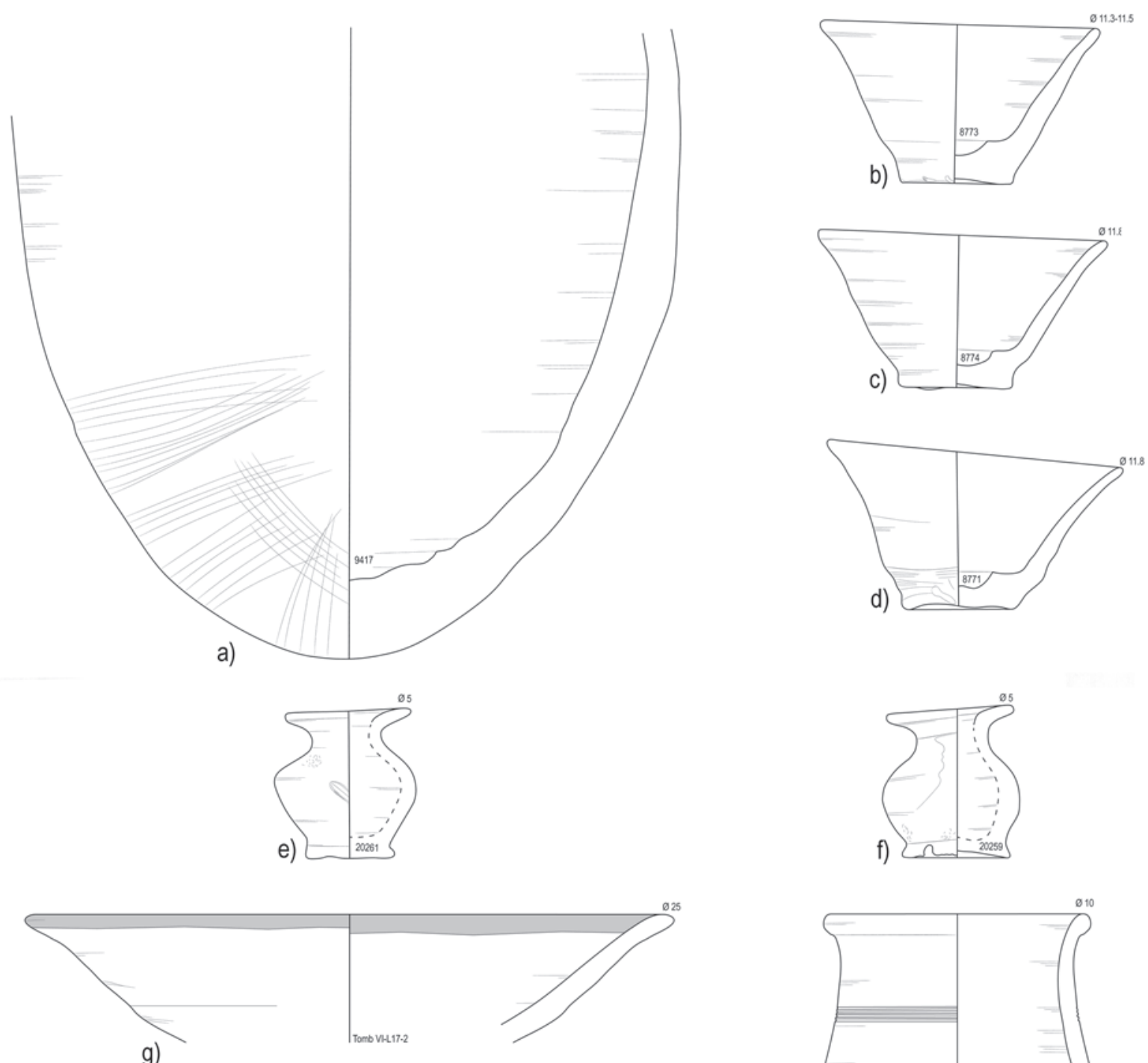

g)
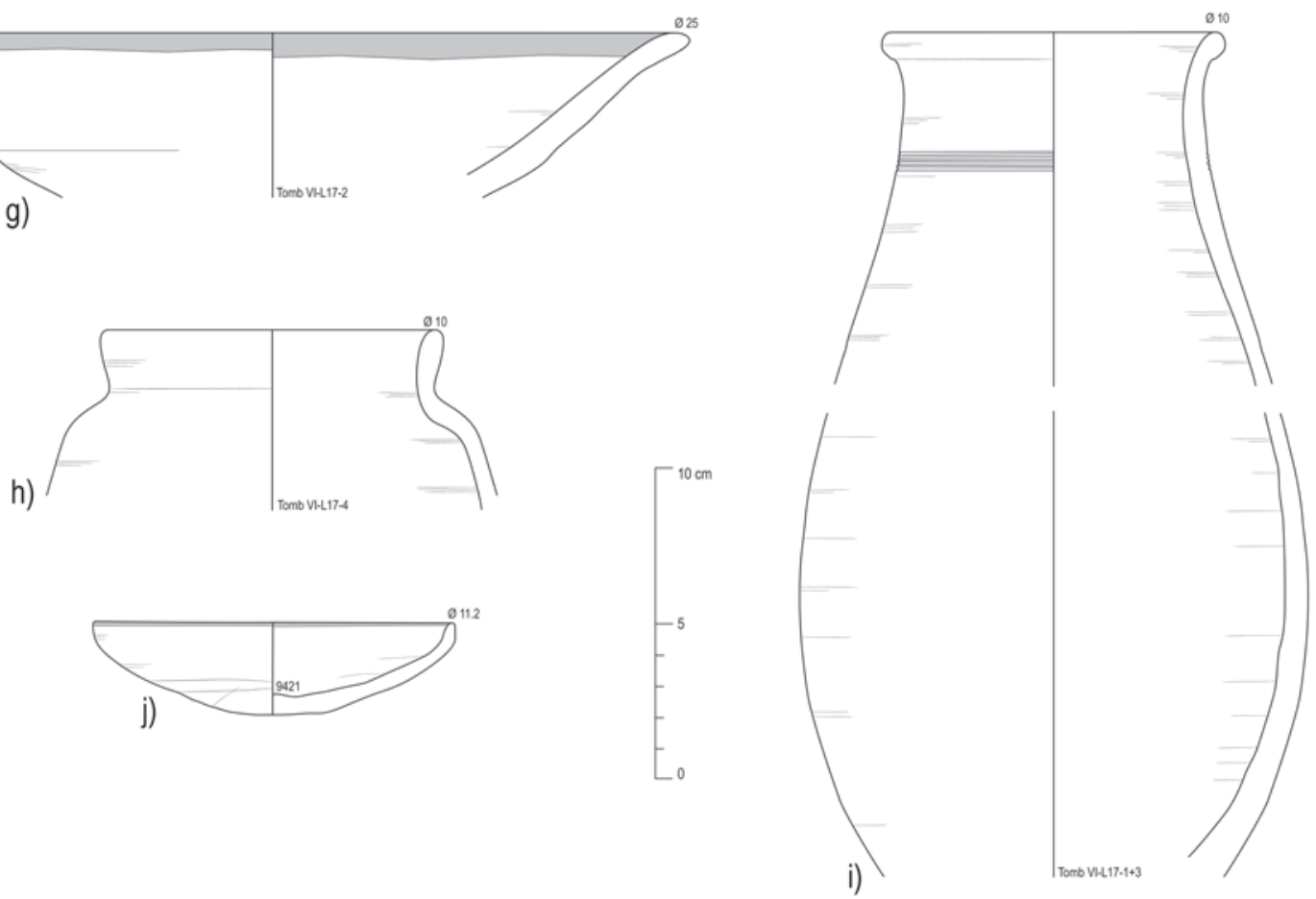

Fig. 23 a) Pottery from Tomb VI-L12-17, late Middle and b-f) Pottery of Tomb VI-L16, late Middle Kingdom, g-i) Pottery of Tomb VI-L17, New Kingdom, j) Pottery of Tomb VII, New Kingdom, drawn by B. Bader, inked by E. Schuster. 
The only diagnostic material of New Kingdom date came from the material at the entrance of the burial chamber, L17, and can be assigned to the early to mid- $18^{\text {th }}$ Dynasty. The undiagnostic material also yielded some New Kingdom sherds of closed vessels, most probably belonging to 'beer jars' of Holthoer's types BB2 or BB3 as these are the most frequent types encountered in pure NK contexts across the site.

\section{Nile B2 - Shallow dish with direct out-turned rim (Fig. 23.g)}

Fabric as well as shape and morphological details suggest a New Kingdom date.

Tomb VI-L17-2, rd.= 26, pres.ht.= 5.2, Nile B2, surface uncoated with red slipped rim band on interior and exterior, wheelmade, base horizontally trimmed with tool on wheel.

\section{Nile B2 - Shouldered BB jar (Fig. 23.h)}

This sherd belongs to Holthoer's type $4^{188}$ and it embodies one of the few fragments with shoulder from the Thutmosis III temple. The vast majority of the other BB jars follows the direct incurved shape. Whether the fragment found in the fill of the burial chamber indicates a later date than Thutmosis III must remain uncertain.

Tomb VI-L17-4, rd.= 10.0, pres.ht.= 5.7, Nile B2, surface uncoated, wheelmade (W1).

\section{Marl A4 - Slender jar (Fig. 23.i)}

This vessel was reconstructed from non-joining sherds, but they were the only marl clay fabric fragments in the context. The shape is typical for the early New Kingdom especially with the horizontal grooves below the rim. ${ }^{189}$

Tomb VI-L17-1+3, rd.= 10.0, max.d. $=$ ht. $=11.3$ +14.8 , Marl A4, section uniform greenish, surface covered with scum, wheelmade, five horizontal grooves, pre-firing, base finished on wheel.

\subsection{6.) Tomb VII (960-970-980-South 4)}

Tomb VII was re-excavated in 2011 by M. Seco. It had previously been excavated by A. Weigall. ${ }^{190}$ It

\footnotetext{
188 HOLthoer 1977, pl. 18.

189 Aston 2003, Fig. 1b, lower row; fig. 1c in the middle.

190 See plan Weigall 1907, 286.

191 Seco Álvarez 2015, plan 5; Seco Álvarez et al. in press, ms 10, plan 4

192 Brunton 1930, pl. XIII, type 9q, r, s, t, u. No exact parallel exists.
}

is currently used to store ceramic material in stackable plastic (vegetable) boxes covered with reed mats. The material found inside the chamber, the shaft and the corridors was certainly backfill consisting of surface debris contemporary with Weigall's work. ${ }^{191}$ For this reason after proper processing only exceptional material was marked for recording by drawing. This included a small dish with an animal foot print, no. 9421, as well as a Marl A4 footed carinated bowl with elaborate prefiring incisions on the interior and false spouts and incised wavy lines on the exterior as is known from the 'Second Intermediate Period' corpus of Qau and Badari III. ${ }^{192}$ A shallow dish made of a fine Nile B1/2 with white dots painted with the fingers was found there as well as a nicely made red polished high stand as found in tomb III (see Fig. 11.1).

The back fill of the shafts and chamber was reexcavated in four 'layers' (L1, L2, L4 and L5) and the ceramic material dates to the late Middle Kingdom, the New Kingdom (probably up to the $19^{\text {th }}$ Dynasty), Late Antiquity (LRA 7, not specifically diagnostic, $2^{\text {nd }}$ to $8^{\text {th }}$ century $\mathrm{AD}^{193}$ ), and the modern period (represented by a few ballâs jar fragments with incised patterns). Among the sherds in layer 1 and 4 there were also some blue painted ones. ${ }^{194}$ In this back fill a number of mud stoppers have also been found. Both conical and dome shaped stoppers derived from vessels with a rim diameter of about $7.0-9.0 \mathrm{~cm}$ were documented. ${ }^{195}$

\section{Nile B2 - Model dish (Fig. 23.j)}

According to Seiler's work at Dra Abu el-Naga shapes like this occur from the late Second Intermediate Period (' $17^{\text {th }}$ Dynasty') and continue into the New Kingdom. The vessel from Tomb VII in contrast does not show a white wash which seems to be very common for the Dra Abu el-Naga examples. ${ }^{196}$

No. 9421 (L4), rd.=11.2, ht.= 3.5, Nile B2, section uniform light brown, surface uncoated (10R7/3 very pale brown), wheelmade, base cut from wheel with tool and trimmed, animal footprint in the interior of the dish.

\footnotetext{
193 Cf. for example Pyke 2005.

194 The patterns include wide petals and stripes and narrow petals.

195 For more details see Bader in Seco Álvarez et al. in press.

196 SeILER 2005, 140-141, Fig. 62.1-2, 4.
} 
a)
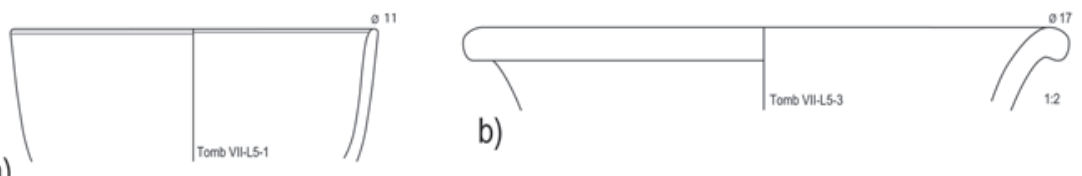

b)
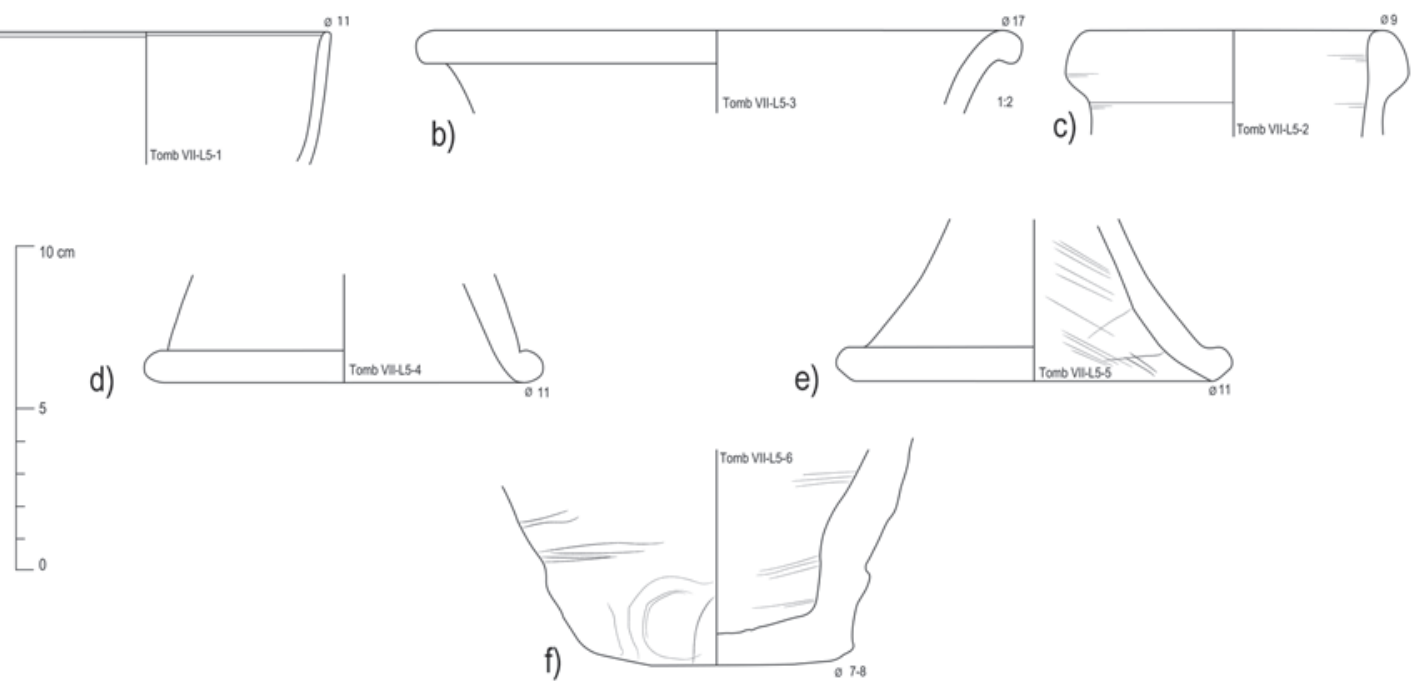

Fig. 24 Pottery from Tomb VII-L5, funerary chamber, Middle and New Kingdom, drawn and inked by B. Bader.

Layer Tomb VII-L5 represents the material recovered from the funerary chamber and only Middle and New Kingdom sherds were found. Because nothing later in date was mixed in there might be a very slight possibility that this material was originally laid down in the funerary chamber which may not have been cleaned thoroughly by Weigall. However, it is safer to assume that the material is from the back-fill.

\section{Nile B2 - Hemispherical cup (Fig. 24.a)}

The hemispherical cup is of open shape with a rather straight vessel contour, with a red rim band.

Tomb VII-L5-1, rd.= 11.0, pres.ht.= 4.0, Nile B2, surface uncoated with thin red slipped rim band, coiled and turned.

\section{Nile B1/2 - Ring stands (Fig. 24.b, d-e)}

The stand fragments belong to the usual range for supports in the Middle Kingdom. ${ }^{197}$

Tomb VII-L5-3, rd.= 17, pres.ht.= 2.5, Nile B1, surface uncoated, coiled and turned, well smoothed.

Tomb VII-L5-4, bd.= 11.0, pres.ht.= 3.4, Nile B1, surface uncoated, coiled and turned, well smoothed.

Tomb VII-L5-5, bd. $=11.0$, pres.ht. $=5.0$, Nile B2, surface burnt, coiled and turned, interior of base scraped with a tool.

\footnotetext{
197 SCHiestl/Seiler 2012, 820-822.

198 SEILER 2005, 70-75.
}

Nile C2 - 'Beer' jar (Fig. 24.c)

The beer jar rim belongs to the type with a bulbous folded lip and a very slight indentation on the interior, belonging to Seiler's type DAN 2. ${ }^{198}$

Tomb VII-L5-2, rd.=9.0, pres.ht.= 3.3, Nile C2, surface uncoated, coiled and turned.

\section{Nile B2/C1 - BB jar (?) of the New Kingdom} (Fig. 24.f)

Tomb VI-L5-6, bd. $=7.0-8.0$, pres.ht. $=7.0$, Nile $\mathrm{B} 2 / \mathrm{C} 1$, surface uncoated, base string cut with finger impressions.

\subsection{7.) Tomb IX (square 960 -North 1)}

The tomb was excavated in 2012 by Abd el Ghani Abd el-Rahman. The rectangular tomb shaft had a depth of $3.4 \mathrm{~m}$ and two tomb chambers, one opening towards the east and one towards the west. ${ }^{199}$

The ceramic material from Tomb IX was drawn and analysed in full. All the Layers (L9, L5, L6, L7 and L8) belonging to this tomb contained mixed ceramic material, mostly from the late Middle Kingdom with a much smaller component dating to the New Kingdom often restricted to single body sherds, e.g. in L8. Taken together there was not too much material but it can be considered typical for a late Middle Kingdom tomb assemblage and includes at least one Nile $\mathrm{C} 2$ 'beer jar' body

199 Seco Álvarez 2015, plan 1 for the location of the tomb in the temple. Seco Álvarez et al. in press, ms 5, plan 5. Seco Álvarez/Martinez Babon 2015b, 37. 
preserved up to the neck (no. 8682, Fig. 26.j) as well as a large dish also made of Nile C2. The ubiquitous Middle Kingdom beer jar body fragments could not be reconstructed despite prolonged efforts to do so. The grand total of 320 body fragments belonging to 'beer jars' amounts to $24.0 \mathrm{~kg}$. They were already extremely eroded, as if they had been exposed to water or wind. Thus, it might be suggested that the tomb shaft and chamber were open at least for a while to allow weather related phenomena to affect the pottery fragments thoroughly. Flash floods filling the shaft and the chamber are also possible.

960-N1-L9 signifies a surface context close to the mouth of the shaft but not exactly belonging to the tomb. The other assemblages are shaft and burial chamber fill. Interestingly Tomb IX-L5 also contained one single very much later sherd (tightly ribbed $^{200}$ ) probably dating to the Third Intermediate Period or later. The material was thoroughly processed and reconstructed. Cross joins were found between L6 and L7, and L3 and L5. Thus the ceramic material will be presented in the groups L9, L5, L6 and L7, L8.

The topmost pottery assemblage 960-N1-L9 already yielded a good proportion of Middle Kingdom ceramic material with only a few New Kingdom diagnostics (a clear flower pot rim with a small lip (960N1-L9-1), the base of a 'BB jar' and the base of a Marl A4 jar (960N1-L9-23)). There was even a dish fragment that may belong to the early Middle Kingdom corpus (960N1-L9-11) with a deep horizontal groove under the orifice plane on the interior of the vessel. This context contained the most of the New Kingdom 'BB jar' sherds of all the assemblages of the tomb.

\section{Nile B1 and B2 - Hemispherical cups (Fig. 25.a-b)}

There were two Nile B1 hemispherical cups in the assemblage with a rim diameter of $12.0 \mathrm{~cm}$ and a thin red slipped rim band. Their shape was open. The Nile B2 fragment has the same dimensions, shape and surface treatment. The preserved base (no 960N1-L9-7) was trimmed with a tool and very well smoothed on the interior. See previous discussions of this type.

\footnotetext{
200 This sherd joined another from 960-North 1-L3 which represents material from the surface of the square.

201 Schiestl/Seiler 2012, 962-964, are similar but not exactly the same.
}

960N1-L9-4, rd.= 12.0, pres.ht. $=4.1$, Nile B1, surface uncoated with red slipped rim band, coiled and turned.

960N1-L9-7, pres.ht. $=2.5$, Nile B1, surface uncoated, coiled and turned, base trimmed with tool.

\section{Nile B2 - Model jar ('canopic', Fig. 25.c)}

The full profile of a model jar was made from Nile B1. Similar shapes occurred already before (Fig. 20.i) but there are no other comparanda from the Theban necropolis. ${ }^{201}$

960N1-L9-1, rd. $=4.0$, max.d. $=7.1$, ht. $=7.5$, Nile B2 fine variant, section uniform light brown, surface uncoated, coiled and turned, base string cut, lower part of vessel squeezed and turned.

\section{Nile B2 - Carinated dish with grooves (Fig. 25.d)}

This rim fragment just preserves the carination that starts quite high up creating only a short vertical contour. The two horizontal grooves were incised pre-firing on the exterior just below the rim. The rim diameter suggests a rather small vessel although the measurement was not certain. ${ }^{202}$

960N1-L9-8, rd. $=9.0$ ?, pres.ht. $=2.4$, Nile B2, surface light red slipped on exterior, coiled and turned (?).

\section{Nile B2 - Base of open form (Fig. 25.e)}

One ring base was made on the wheel which may be an indication that the sherd should be dated to a later period than the Middle Kingdom, probably the late Second Intermediate Period or more likely the New Kingdom. Another dish base with a red slip on the interior and exterior was, in contrast, hand modelled (bd. $=4.5$, Nile B2).

960N1-L9-3, bd. $=4.0$, pres.ht. $=2.2$, Nile B2, surface burnt, wheelmade (?), interior smoothed.

\section{Nile B2 - Large dish with incised groove (Fig. 25.f)}

This rim fragment of a very shallow dish is very reminiscent of an older tradition in pottery making perhaps even derived from the late Old Kingdom. ${ }^{203}$ The type and quality of the red slip supports this impression. It is possible of course to

\footnotetext{
202 Schiestl/Seiler 2012, 230-234 with varying lenghts of carinations, 238, no 9.

203 Seidlmayer 2005, Abb. 1. 61BD/2
} 

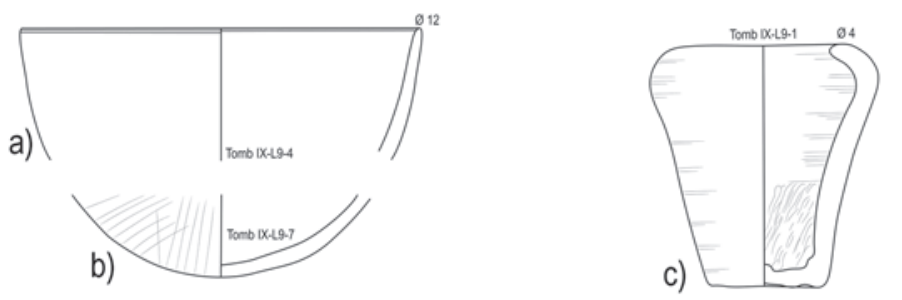

d)
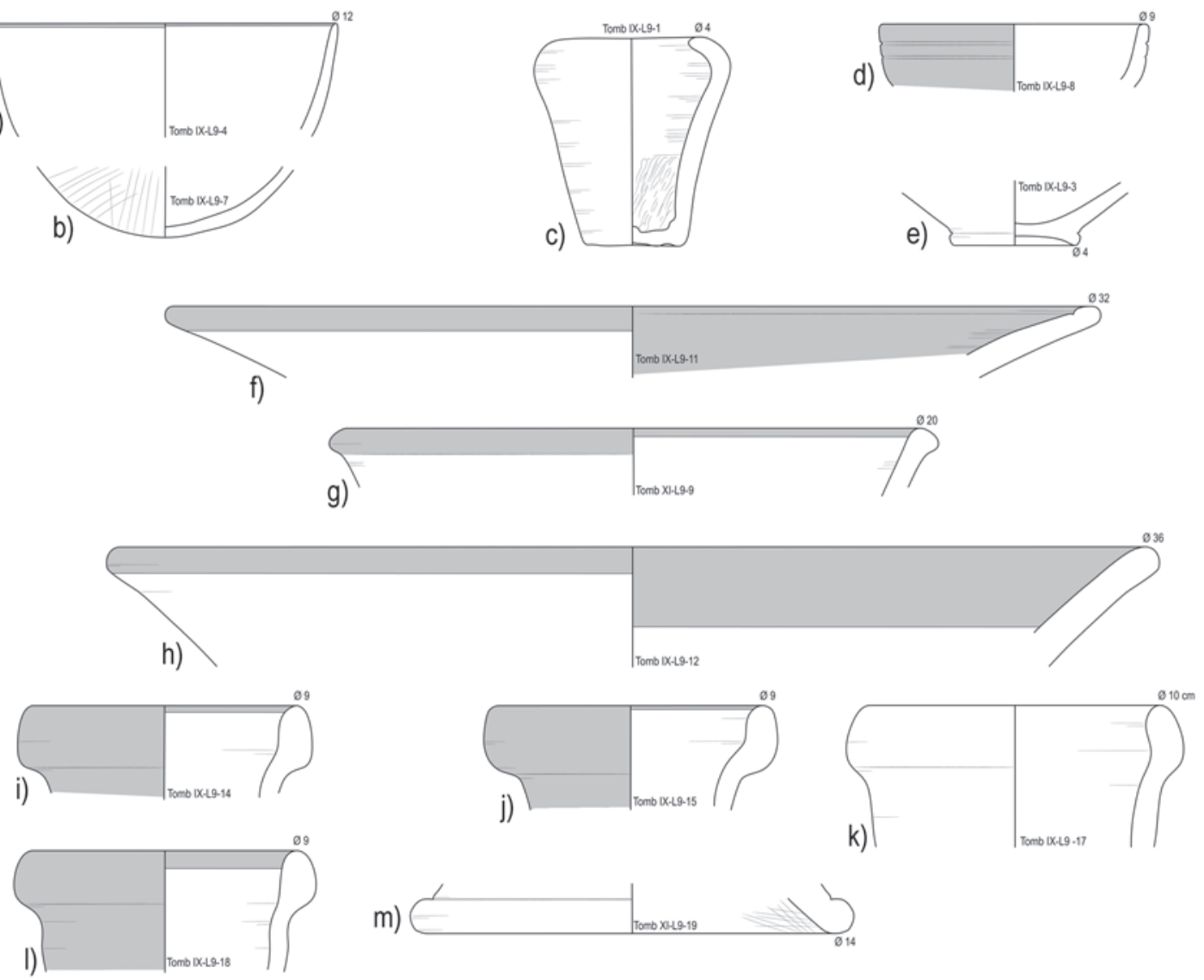

$\mathrm{m})$
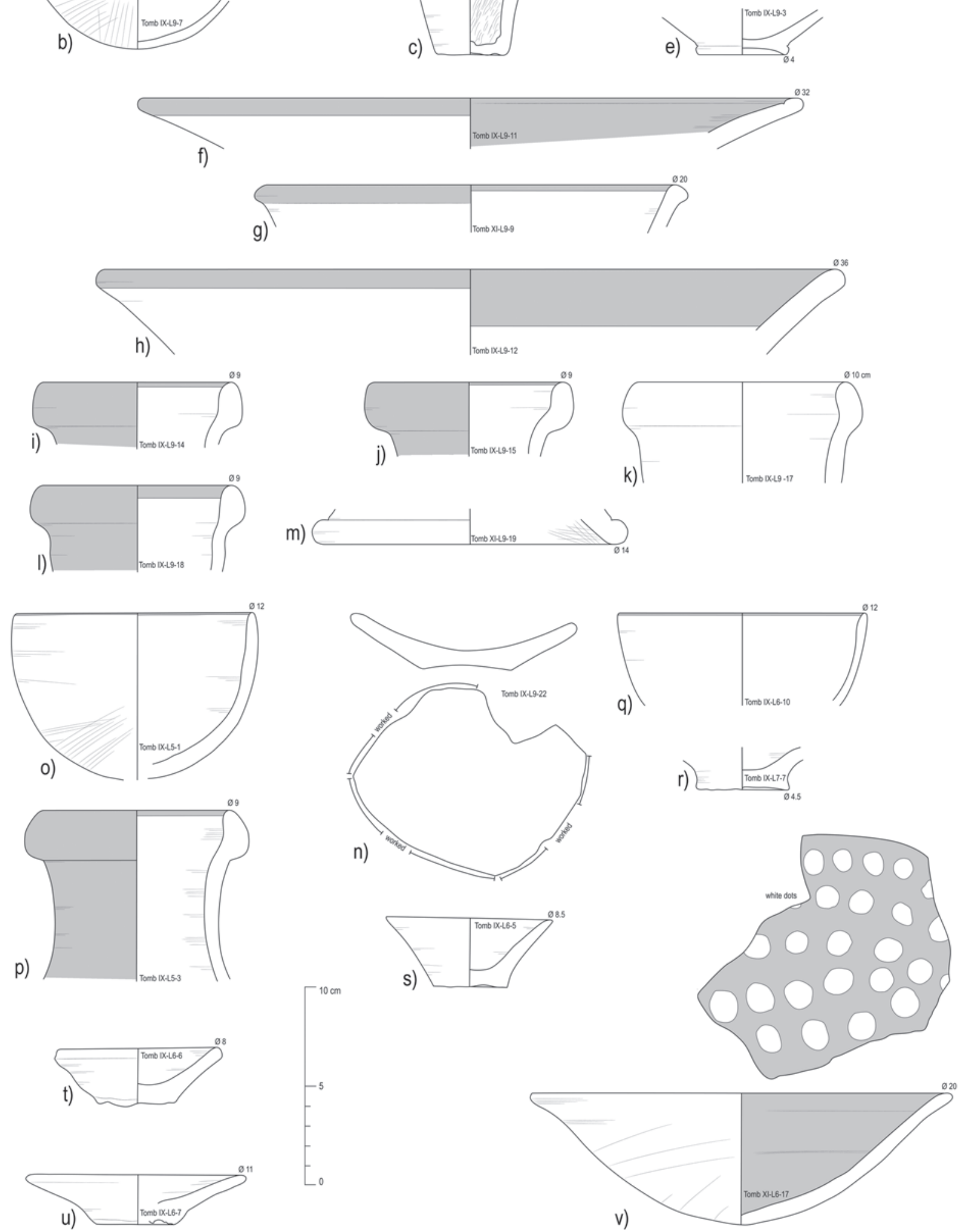

Fig. 25 a-n) Pottery from Tomb IX-L9. o-p) Pottery from Tomb IX-L5. q-v) Pottery from Tomb IX-L6 and L7, late Middle and New Kingdom, drawn by B. Bader, inked by E. Schuster. 
find remnants of the earlier history of the Theban necropolis throughout.

960N1-L9-11, rd.= 32.0?, pres.ht. $=2.5$, Nile B2, surface red slipped on interior and on exterior rim, coiled and turned (W1?).

\section{Nile C2 - Medium and large dishes and bowls} (Fig. 25.g-h)

Whilst two examples of dishes or bowls with direct rim occur (Fig. 25.h), once with a red slip on the interior and rim on exterior and once covered in white plaster $(960 \mathrm{~N} 1-\mathrm{L} 9-10, \mathrm{rd} .=37.0$, not shown), the other type (Fig. 25.g) shows a thickened and modelled rim with a red rim band on the interior and exterior. Such dishes occur frequently during the whole Middle Kingdom. ${ }^{204}$

960N1-L9-9, rd. $=20.0$, pres.ht. $=$ Nile C2, surface red slipped rim on exterior and interior, coiled and turned.

960N1-L9-12, rd. $=36.0$, pres.ht. $=$ Nile C2, surface red slipped on interior and rim exterior, coiled and turned.

\section{Nile C2 - 'Beer' jars (Fig.25.i-l)}

All preserved fragments show an indentation on the interior of the rim, without the possibility to judge if indeed a kettle rim was intended. The rim diameters are quite uniform with 9.0 and $10.0 \mathrm{~cm}$. The shape of the necks may have been tubular or slightly funnel shaped. ${ }^{205}$

960N1-L9-14, rd. $=9.0$, pres.ht. $=3.2$, Nile C2, surface red slipped on exterior and rim on interior, coiled and turned.

960N1-L9-15, rd.=9.0, pres.ht. $=3.8$, Nile C2, surface red slipped on exterior and rim on interior, coiled and turned.

960N1-L9-16-17, rd. $=10.0$, pres.ht. $=5.1$, Nile C2, surface burnt, coiled and turned.

960N1-L9-18, rd. $=9.0$, pres.ht. $=4.4$, Nile C2, surface red slipped on exterior and rim on interior, coiled and turned.

\section{Nile C2 - Stand (Fig. 25.m)}

960N1-L9-19, bd. $=14.0$, pres.ht. $=1.8$, Nile C2, surface uncoated, coiled and turned, base scraped on interior.

Interestingly a secondary tool made from a New Kingdom dish was also found in this assemblage (Fig. 25.n).

\footnotetext{
204 Schiestl/Seiler 2012, 72-73.

205 SEILER 2005, 70-75, DAN 2 and 3.

206 Schiestl/Seiler 2012, 100-104.
}

960N1-L9-22, bd. $=4.5,11.8$ by 9.4, Nile B2, surface dark red slipped on exterior and interior, wheelmade.

Ceramic material of Tomb IX-L5 was collected at the mouth of the shaft. Late Middle Kingdom (probably early to mid-13 ${ }^{\text {th }}$ Dynasty) material was mixed with pieces from the surface dating to the New Kingdom and one body sherd (fast wheel, tightly ribbed) dates clearly to the $8^{\text {th }}$ century $\mathrm{BC}$ or later. Five examples of New Kingdom BB jar bases and two rim fragments as well as a jar base with horizontal deep grooves from working the base on the wheel were present as well as rim fragments of flower pots. Only the diagnostic Middle Kingdom pottery will be shown here that does not occur already in the corpus of L9.

\section{Nile B1 - Hemispherical cup (Fig. 25.o)}

One almost complete profile of a cup with straight vessel contour and a rim diameter of $12.0 \mathrm{~cm}$ belongs to this small context. The thick vessel wall is noteworthy. ${ }^{206}$

$960 \mathrm{~N} 1-\mathrm{L} 5-1, \mathrm{rd} .=12.0$, max.d. $=12.4$, pres.ht. $=$ 8.4, Nile B1, surface uncoated with red slipped rim band, coiled and turned, base trimmed with a tool. VI $\sim 148$.

\section{Nile C2 - 'Beer' jars (Fig. 25.p)}

The kettle rim with a medium long neck is similar to examples already described for $\mathrm{L} 9 .{ }^{207}$

960N1-L5-3, rd.=9.0, pres.ht. $=8.6$, Nile C2, surface red slipped on exterior and on rim interior, coiled and turned.

The two contexts Tomb IX-L6 and Tomb IX-L7 were combined for presentation because at least two different vessels were joined together by sherds. Both contexts contained late Middle Kingdom and New Kingdom pottery, where the majority belongs to the Middle Kingdom. Especially noteworthy is the quite well preserved body of a 'beer jar' from L6 (Fig. 26.j). The 'beer jar' rim fragments found in L6/L7 (Fig. 26.b-i) indicate a $13^{\text {th }}$ Dynasty or early Second Intermediate Period date $^{208}$ although other pottery suggests a date in the later Second Intermediate Period. Perhaps two phases of usage are represented in the material remains of this tomb.

\footnotetext{
207 SeILER 2005, 70-75, DAN 3.

208 SeILER 2005, 70-75, 127-130.
} 


\section{Nile B1 - Hemispherical cup (Fig. 25.q)}

This cup fragment represents a moderately open shape with a thin red rim band. ${ }^{209}$

Tomb IX-L6-10, rd.= 12.0, pres.ht. $=4.6$, Nile B1, surface uncoated with thin red slipped rim band, coiled and turned, well smoothed.

\section{Small Dishes, various fabrics (Fig. 25.r-u)}

A variety of small dishes and plates was found. There are none of the types among them that are put by Seiler in the latter part of the Second Intermediate Period or even the New Kingdom. ${ }^{210}$

Tomb IX-L7-7, bd. $=4.5$, pres.ht. $=2.2$, Nile B1, surface uncoated, base string cut.

Tomb IX-L6-5, rd. $=8.2$, bd. $=3.7$, ht. $=3.6$, Nile B2, surface uncoated (7.5YR7/3 pink), coiled and turned (W1?), base string cut, good quality.

Tomb IX-L6-6, rd. $=8$, bd. $=4.0$, ht. $=2.8$, Nile C2, surface uncoated (10YR6/4 light yellowish brown), coiled and turned, base string cut, bad quality, rim trimmed with tool or fingers.

Tomb IX-L6-7, $\mathrm{rd} .=11.0, \mathrm{bd} .=4.0, \mathrm{ht} .=2.6$, Nile C2, surface uncoated (10YR7/3 very pale brown), coiled and turned (W1?), base string cut.

\section{Nile B2 - Dish with white dots (Fig. 25.v)}

The dish with white dots represents one of the more problematic vessel types in the repertoire of the tomb. Shape, technology and quality of the vessel resembles very closely those of the Middle Kingdom as represented in Tomb III-L17 (Fig. 9.d with dots and Fig. 9.e and g), which suggests a date in the earlier part of the Middle Kingdom but not the late part or even the Second Intermediate Period. ${ }^{211}$ Thus, it is difficult to decide whether these dishes might form a group with the beaker jar no. 8801. It is possible but cannot be proven from the current find situation.

Tomb IX-L6-17, rd. $=20.0$, max.d. $=21.2$, ht. $=$ 6.6, Nile B2 fine variant, surface red slipped on interior 2.5YR6/4 weak red), uncoated on exterior, on interior white dots painted with finger tips, the white colour reacted to lemon juice, thus the paint is lime based, coiled and turned, with upper part joined on separately, interior very well smoothed, base trimmed and smoothed subsequently.

\footnotetext{
209 Schiestl/Seiler 2012, 96-104.

210 Cf. Seiler 2005, 78-79, 140-141.

211 SeIler 2005, 80-81, Abb. 34. But their shape and technology is different.
}

Nile C2 - Basin with squared rim (Fig. 26.a)

Several fragments of this basin were found in L6 and L7 and two pieces joined those contexts. The rim diameters range from 24.0 to $32.0 \mathrm{~cm}$, which exemplifies the irregularity of these vessels made in combination technique. The rim fragments remained uncoated. Their very sharp trimming of the rim by means of a tool is a unique feature of these fragments. ${ }^{212}$

Tomb IX-L6-1-4, $\mathrm{rd} .=24.0$, pres.ht. $=5.0$, Nile $\mathrm{C} 2$, surface uncoated, coiled and turned, sharp trimming of edge of rim.

\section{Nile C2 - 'Beer' jars (Fig. 26.b-j, 27.a)}

At least 6 beer jar bases (in total $5.5 \%$ estimated base vessel equivalents ${ }^{213}$ ) were found left in the tomb and it seems quite clear that they should have belonged to the original tomb assemblage (Tomb IX-L6-21-25, no. 8682). The rim fragments of the beer jars belong partly to the so-called 'kettle-rim' (Fig. 26.b, d-j) and partly to a very vertical rimneck type with only a slight indentation on the interior (Fig. 26.c). ${ }^{214}$ One better preserved example has a neck that is relatively short but also quite round. Almost all the rim fragments have a rim diameter/aperture of about $9.0 \mathrm{~cm}$. The majority of the fragments were identified as Nile $\mathrm{C} 2$.

\section{Bases}

No. 8682 , max.d. $=30.5$, pres.ht. $=45.3$, Nile $\mathrm{C} 2$, section wide dark grey core, red and brown oxidation zones, surface red slipped on exterior (10R $5 / 6$ red), natural surface colour (10YR7/3 very pale brown), coiled and turned on turning device/wheel (W1), base roughly trimmed with a tool. The vessel was filled with earth just the same as the fill of the tomb, with some remnants of very fine mud and lime plaster (lemon test). There were a few sherds of the vessel itself in the fill. The rim is missing, but the body is preserved up to the neck.

Tomb IX-L6-25, pres.ht. $=22.2$, Nile C2, surface red slipped on exterior, coiled and turned, base roughly scraped on exterior, made in at least two parts (join line), held together while drying by rope.

\footnotetext{
212 Schiestr/Seiler 2012, 316, no 3-4 but the current example is more out-turned than incurved.

213 Orton et al. 1993, 171-173.

214 SEILER 2005, 70-75, DAN 2 and 3.
} 

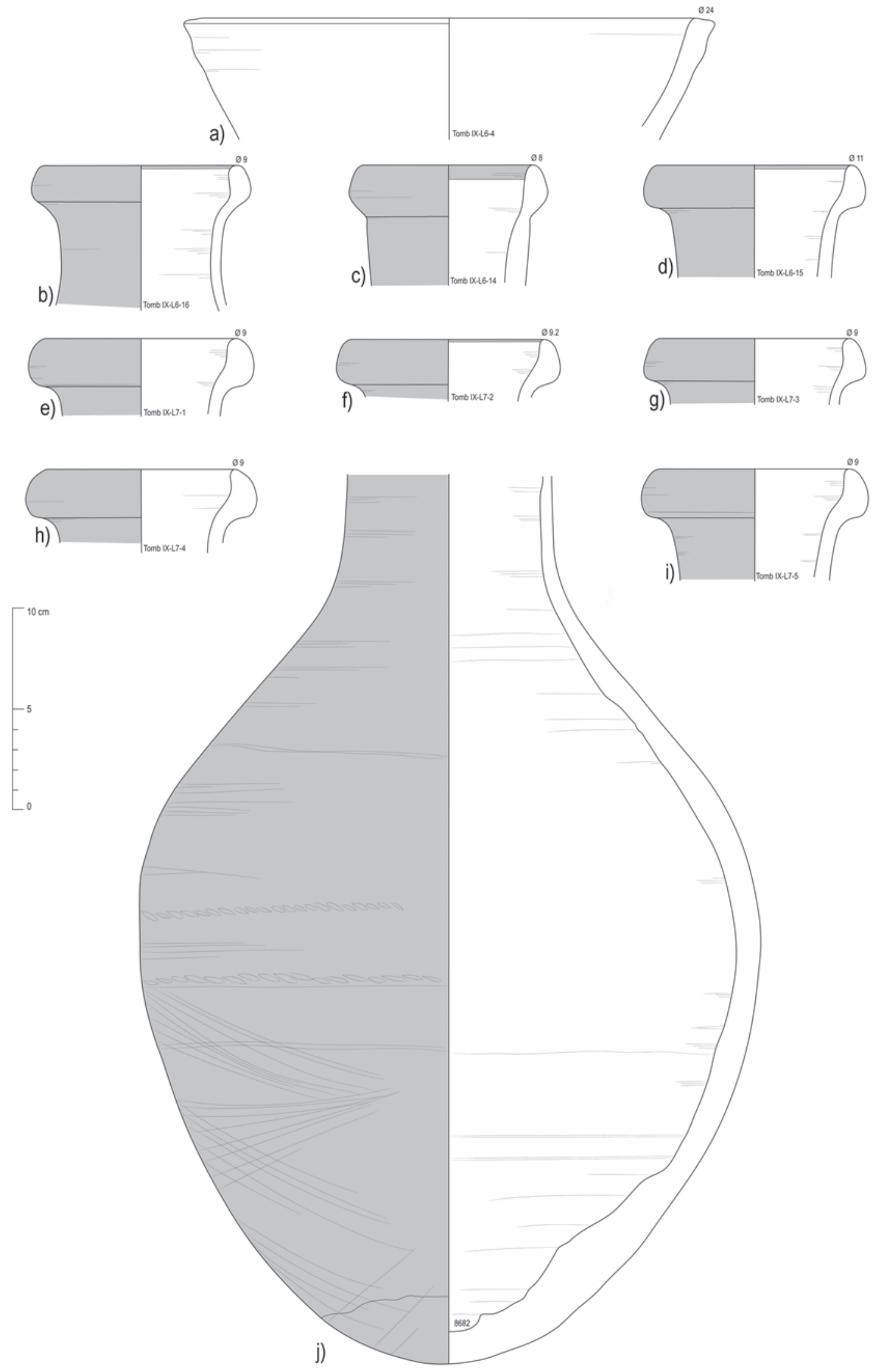

Fig. 26 Pottery from Tomb IX-L6 and L7, late Middle Kingdom, drawn by B. Bader, inked by E. Schuster. 


\section{Rims}

Tomb IX-L6-16, rd.= 9.0, pres.ht.= 7.2, Nile C2, surface red slipped on exterior and interior on rim, coiled and turned.

Tomb IX-L6-14, rd.=8.0, pres.ht.= 5.8, Nile C2, surface red slipped on exterior and interior on rim, coiled and turned, same as no. 13.

Tomb IX-L6-15, rd.= 11.0, pres.ht.= 5.6, Nile C2, surface red slipped on exterior and interior on rim, coiled and turned, same as no. 12 with $\mathrm{rd} .=9.0$.

Tomb IX-L7-1, rd.=9.0, pres.ht.= 3.8, Nile C2, surface red slipped on exterior, coiled and turned.

Tomb IX-L7-2, rd.= 9.2, pres.ht.= 3.1, Nile C2, surface red slipped on exterior and interior on rim, coiled and turned.

Tomb IX-L7-3, rd.= 9.0, pres.ht.= 3.3, Nile C2, surface red slipped on exterior, coiled and turned.

Tomb IX-L7-4, rd.=9.0, pres.ht.=4.1, Nile C2, surface red slipped on exterior, coiled and turned.

Tomb IX-L7-5, rd.=9.0, pres.ht.= 5.5, Nile C2, surface red slipped on exterior, coiled and turned.

\section{Nile B2 - Beaker jar (Fig. 27.b)}

This beaker jar is perhaps also representative of the later usage of this tomb. The vessel was wheelmade and joined together in at least two parts, but the fabric of the vessel is much harder than the average Middle Kingdom Nile B2 and the shape does not belong to the repertoire of the late Middle Kingdom in Thebes or elsewhere. It remains doubtful, however, that this single vessel represents a use phase of Tomb IX in the late Second Intermediate Period although there are formal parallels at Dra Abu el-Naga. ${ }^{215}$ It is possible that it came into the tomb at the same time as the fragmented New Kingdom material.

No. 8801 (L6+7), rd.=8.0, max.d.= 10.8, ht.= 22.8, Nile B2, section pink core with red and orange oxidation zones, surface uncoated (7.5YR6/3 light brown), wheelmade (W1), joined from at least two parts, base trimmed and smoothed subsequently, post-firing hole cut into the base but not in centre.

\section{New Kingdom jar with folded rim (Fig. 27.c)}

Although this is the only certain intrusive diagnostic sherd of the New Kingdom, it shows the mixing of the contents of this tomb.

215 SeIler 2005, 86, Abb. 37. It also remains to be seen, if the difference in technology - wheelmade (W1) as opposed to wheelmade with folds on the interior from squeezing together may be reminiscent of a chronological difference
Tomb IX-L7-6, rd.=9.0, pres.ht.=4.0, Nile B2/ $\mathrm{C} 1$, surface uncoated, wheelmade (W1).

Finally Tomb IX-L8 is the last find context in this tomb with pottery to be presented. In this context the New Kingdom component was represented by only one body sherd of a closed vessel with horizontal black lines on a red slip - the hall mark of the early $18^{\text {th }}$ Dynasty (not shown).

\section{Nile B1 - Hemispherical cup (Fig. 27.d)}

The only hemispherical cup rim fragment of this assemblage is open in shape with a slightly turned out rim and a thin red slipped rim band. The rim diameter is, with $13.0 \mathrm{~cm}$, on the wider end of the scale of such cups. It is very similar to that of Fig. 25.q.

Tomb IX-L8-1, rd.= 13.0, pres.ht.= 3.0, Nile B1, surface uncoated with a thin red rim band, coiled and turned.

\section{Nile B2 - Bowl with horizontal grooves (Fig. 27.i)}

Large bowls with horizontal grooves are often carinated in shape, but this rim fragment is not well enough preserved to determine this. This type seemingly does not occur in the corpus of vessel shapes from Dra Abu el-Naga. This type has already been discussed (cf. Fig. 25.d).

Tomb IX-L8-2, rd.= 31.0, pres.ht.=2.7, Nile B2, surface light red slipped on exterior and interior, coiled and turned.

Nile B2 - Spout (Fig. 27.f)

This quite narrow spout does not allow any conclusions on the vessel type from which it is derived. Many different types comprise spouts. ${ }^{216}$

Tomb IX-L8-3, rd.= 2.0, pres.ht.= 4.8, Nile B2, surface uncoated, handmade: coiled and squeezed.

\section{Nile B2 - Small vessel (Fig. 27.h)}

The extremely thick walled base may have belonged to a model jar rather than an open vessel.

Tomb IX-L8-4, bd.=3.5, pres.ht.= 2.4, Nile B2, surface uncoated, coiled and turned (W1?), base string cut.

or a different choice of workshops. Seiler's figure shows these side by side as if contemporary.

216 Schiestl/SEILER 2012, e.g. 326-327, 336, no 5, 342-345. 

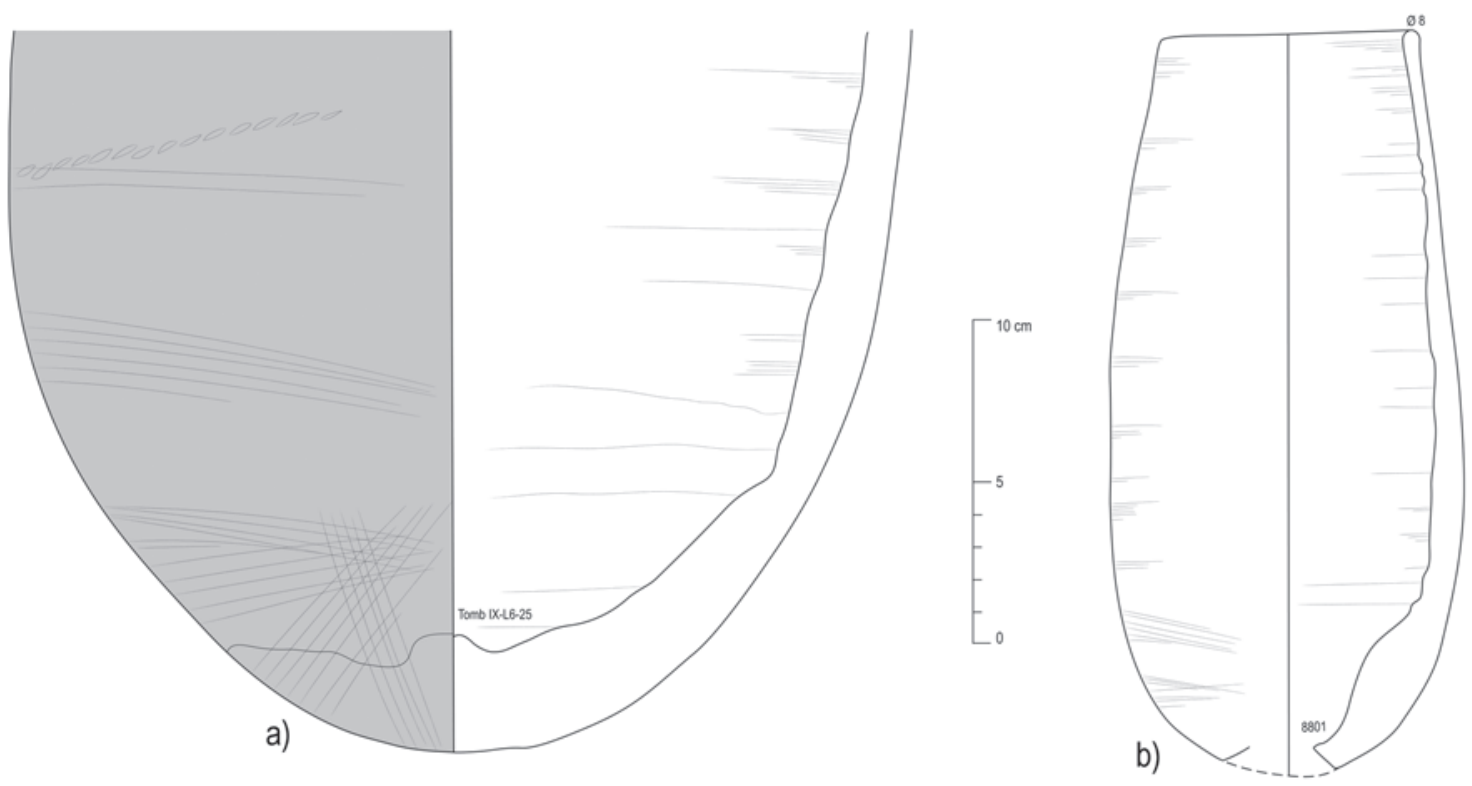
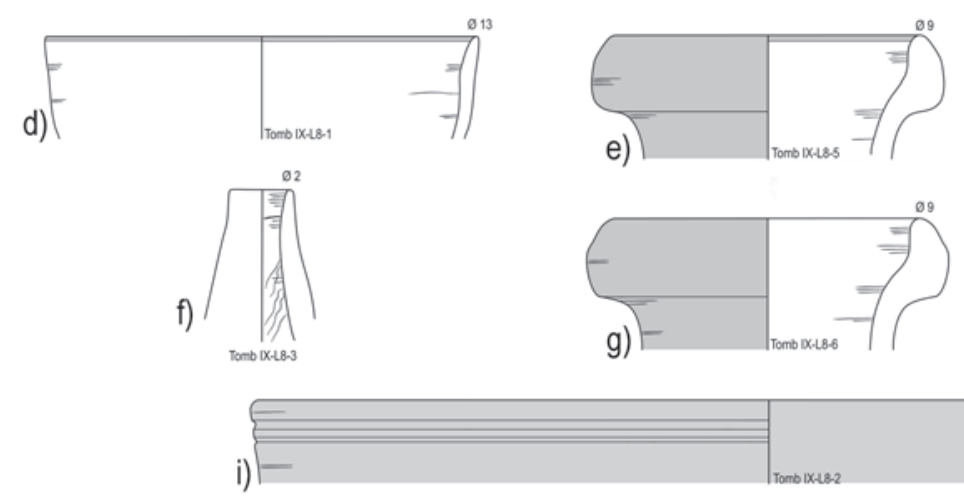

c)

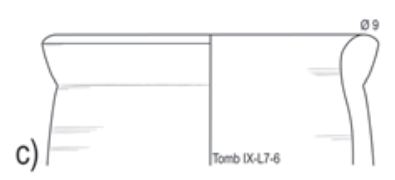

h)
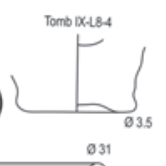

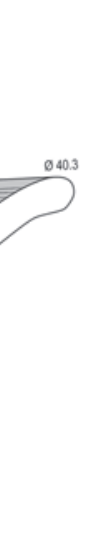

j)

Fig. 27 a-c) Pottery from Tomb IX-L6 and L7, d-j) Pottery from Tomb IX-L8, late Middle and New Kingdom, drawn by B. Bader, inked by E. Schuster.

\section{Nile C2 - Large dish (Fig. 27.j)}

Large dishes are a ubiquitous vessel type in late Middle Kingdom assemblages. The direct rim is slightly out-turned and has direct parallels in the corpus of the late Middle Kingdom/early Second Intermediate Period (' $13^{\text {th }} / 16^{\text {th }}$ Dynasty') from Dra
Abu el-Naga ${ }^{217}$ although the current example is of larger size and better quality. ${ }^{218}$

No. 20107, $\mathrm{rd} .=40.3$, ht. $=10.4$, Nile $\mathrm{C} 2 / \mathrm{C} 4$, section grey core, brown oxidation zones, surface red slipped rim on exterior and partly inside $(10 \mathrm{R} 5 / 6$ red), natural colour (7.5YR6/4 light 
brown), coiled and turned, base coiled and scraped on exterior.

\section{Nile C2 - 'Beer' jars (Fig. 27.e, g)}

Again the existing beer jar fragments belong to the 'kettle' rim variety. From their stance they may have had a slightly funnel shaped neck rather than a tubular one, but preservation is not good enough to be certain.

Tomb IX-L8-5, rd.=9.0, pres.ht.=3.6, Nile C2, surface red slipped on exterior and interior on rim, coiled and turned.

Tomb IX-L8-6, rd.=9.0, pres.ht.= 3.9, Nile C2, surface red slipped on exterior, coiled and turned.

\subsection{8.) Tomb X (square 960-North 2)}

Tomb X was excavated in 2012 and 2013 by Abdel Ghani Abd el Rahman. A deep shaft was found below a mud brick wall belonging to the Temple of Millions of Years covering the mouth of the shaft, which went down $6.30 \mathrm{~m}$. There was a small chamber higher up in the shaft, and two burial chambers to the east and west of the shaft, the lower of which was not examined due to its instability and imminent danger. ${ }^{219}$

Layers L8, L9, L10 and L11 of this tomb mostly came from the shaft and contained late Middle Kingdom as well as New Kingdom pottery. In Layer 11 only a few body sherds of New Kingdom 'BB jars' were found. Due to massive erosion, either by wind or water, they were hard to recognise, which shows the state of the material. Under normal circumstances this distinction does not pose any difficulties. The overall repertoire in all layers is dominated by fragments of Middle Kingdom beer jars that were very eroded and could not be reconstructed to form a complete profile. All together the processing provided a weight of $22.35 \mathrm{~kg}$ for this type. ${ }^{220}$ The remainder of the body sherds comprised a few hemispherical cups and some large Nile C2 dishes and basins (63 fragments with a weight of $1.2 \mathrm{~kg}$ ). Only one beer jar provides a complete profile (no. 20263, Fig. 28.d) due to the fact that it was found articulated. Several additional rim sherds attest to the presence of more examples. Layer 12 contained exclusively

219 Seco Álvarez et al. in press, ms 5-6. Seco Álvarez/Martinez Babon 2015b, 38.

220 The number of sherds was 590 , which suggests a high rate of breakage for this Tomb if compared to the $24.0 \mathrm{~kg}$ and 320 sherds from Tomb IX.
Middle Kingdom material although it was quite eroded, too. Some model vessels were preserved ${ }^{221}$ and could be reconstructed as well as larger parts of basins and large dishes.

Again the eroded state of the pottery poses the question, whether the tomb was open (at the same time as Tomb IX?) for a longer period of time, in order for the surfaces of the assemblage to deteriorate so massively. Hitherto, this feature was not observed in any other tomb assemblages except Tombs IX and X.

No joins were found between layers but the reason for this might be sought in the preservation of the pottery. Therefore, the ceramic material from the layers will be presented in a sequence.

Tomb X-L8, the topmost shaft fill, contained only seven diagnostics among them one unmistakeable New Kingdom 'BB jar' base, the trimmed rim of a 'flower pot' and the handle of a transport amphora. The only other diagnostics were the base of a Nile $\mathrm{C} 2$ (beer) jar and three rim fragments of a large shallow Nile C2 dish covered with white plaster on the interior and the exterior.

The assemblage from Tomb X-L9, also from the shaft, contained several vessels of late Middle Kingdom date with complete profiles. Among these were Nile B2 model dishes, a large Nile C2 dish and one beer jar. The only New Kingdom intrusions were New Kingdom body sherds of 'BB jar' type (1), a Marl A2 vessel (2) and a Canaanite transport amphora (2).

\section{Nile B2 - Small dishes (Fig. 28.a-b)}

The two dishes are distinguished by the visibility of the ridges of the coils or the fast moving of the potter's fingers leaving these ridges without smoothing. Similar features have been detected in the ceramic material of Senebtisi. ${ }^{222}$ The fineness of the fabric makes a New Kingdom date unlikely.

Tomb X-L9-1, rd.=9.0, bd.=3.9, ht. $=2.3$, Nile B2 fine variant, surface uncoated, coiled and turned (W1?), turning spiral visible on interior, base string cut.

Tomb X-L9-2, rd.=9.4, bd.=3.6, ht. $=2.8$, Nile B2 fine variant, surface uncoated, coiled and

\footnotetext{
221 As referred to in the excavation report. Seco Álvarez et al. in press, $\mathrm{ms} 6$.

222 Mace/Winlock 1916, 112.
} 
a)
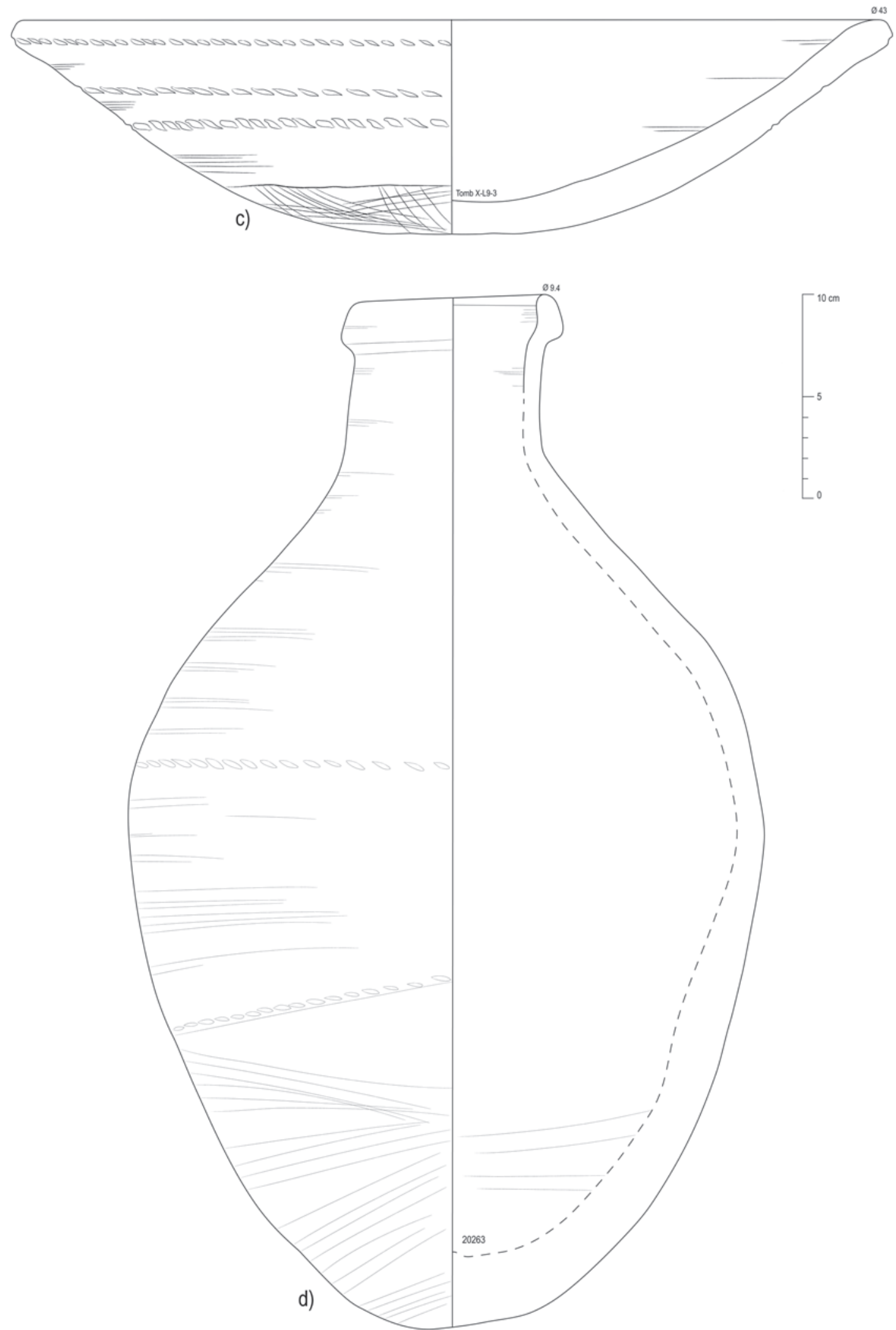

Fig. 28 Pottery from Tomb X-L9, late Middle Kingdom, drawn by B. Bader, inked by E. Schuster. 
turned (W1?), turning spiral visible on interior, base string cut.

\section{Nile C2 - Large dish (Fig. 28.c)}

This large dish shows a dark brown discolouration on the interior around the rim ca 5.0 to $6.0 \mathrm{~cm}$, which may have been a red rim. The interior also contains remains of white colour. These remains were too badly preserved to be tested. Cf. the discussion of this type above (Fig. 27.j).

Tomb X-L9-3, rd.=43.0, ht. $=10.6$, Nile C2, section dark grey core with red and light brown oxidation zones, surface uncoated, coiled and turned, base scraped on exterior, three rows of rope impressions on exterior.

\section{Nile C2 - 'Beer' jar (Fig. 28.d)}

This jar belongs to Seiler's type DAN 3. ${ }^{223}$ See also discussions above.

No. 20263, rd.=9.0, max.d. $=31.1$, ht. $=48.8-$ 49.1, Nile C2, section light grey core and brown oxidation zones, surface red slipped on the exterior (10R5/6 red), natural surface 10YR7/4 very pale brown, coiled and turned, made in at least three parts, base scraped on exterior, two rows of rope impressions on the exterior.

The assemblage of Tomb X-L10 contained again one fragment of an uncoated large shallow dish with direct rim (Nile C2), a Nile C2 basin with flattened base and direct rim (also uncoated), several kettle rims of beer jars (Nile C2), several model jars and small closed vessels in Nile B2, and hemispherical cup base fragments made from Nile B2. Only two New Kingdom intrusive body sherds were present in this context. Again it seems as if the further down in the shaft, the less mixed the material becomes.

\section{Nile B2/C1 - Model vessels (Fig. 29.a-d)}

Beside the base and rims of several closed small vessels that may be classed as models one fragment of a dish or lid was found. The identification as a lid is based on the position of the red rim band that is more visible on the exterior than the interior. Moreover, the rim contour does not conform to most of the other model dishes from the temple. ${ }^{224}$ Flat based jars have been encountered before in

SEILER 2005, 70-75, 127-130.

224 SChiestr/Seiler 20121059, no 3. This type does not occur at Thebes.
Tomb III-L17 (Fig. 11.h), Tomb IV-L2 (Fig. 15.f) and Tomb VI-L12-17 (Fig. 20.1-n).

Tomb X-L10-4, rd./bd.= 7.0, pres.ht.=1.8, Nile B2 fine variant, surface uncoated, red rim band on exterior, coiled and turned (W1?).

Tomb X-L10-10, bd.= 3.3, pres.ht. $=2.6$, Nile B2, surface thick red slipped on exterior, wheelmade (W1), base cut with a tool.

Tomb X-L10-11, rd.=8.0, pres.ht.=3.1, Nile B2/ $\mathrm{C} 1$, surface red slipped on exterior, coiled and turned (W1?), pre-firing hole in vessel wall.

Tomb X-L10-12, rd.=3.5, pres.ht.= 2.5, Nile B2/ $\mathrm{C} 1$, surface uncoated, coiled and turned (W1?), rim trimmed with a tool on interior.

\section{Nile C2 - Large dish (Fig. 29.e)}

See the previous discussions of this type (Fig. 21.b-g, 27.j, 28.c).

Tomb X-L10-1, $\mathrm{rd} .=46.0$, pres.ht. $=8.9$, Nile C4, section dark grey core with light brown oxidation zones, surface uncoated (10YR7/3 very pale brown), coiled and turned, base scraped on exterior, three rows of rope impressions on exterior.

\section{Nile C2 - Large basin (Fig. 29.f)}

This is one of the most complete vessels of this type. A wide band of white wash or slip was applied to the exterior in an irregular manner, with a thin band on the interior. ${ }^{225}$

Tomb X-L10-2, rd. $=45.0$, pres.ht. $=22.9$, Nile $\mathrm{C} 4$, section wide dark grey core with light brown oxidation zones, surface uncoated (10YR7/3 very pale brown), white slipped wide band on exterior (irregular width $3.7-6.0 \mathrm{~cm}$ ) and on interior thin rim band, slip had trickled down, very well smoothed on interior.

\section{Nile C2 - 'Beer' jars (Fig. 29.g-j)}

Again all the beer jar rims found belong to the 'kettle' rim variety. Their rim diameter/aperture varies from 8.0 to $10.0 \mathrm{~cm}$. The fragments were too eroded to tell whether they had a red slip applied or not. The fragments are equivalent to Seiler's type DAN 3, see discussions above.

Tomb X-L10-5, rd.=10.0, pres.ht.=3.5, Nile C2, surface eroded, soft.

Tomb X-L10-6, $\mathrm{rd} .=8.0$, pres.ht.=3.0, Nile C2, surface eroded, soft.

\footnotetext{
25 Schiestl/Seiler 2012, 314-316, 319-320. There is no example with a white slip or wash.
} 
a)

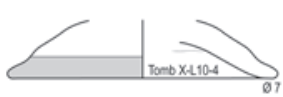

b)

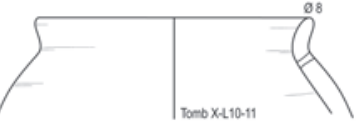

c)

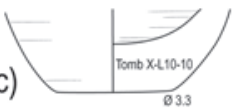

d)

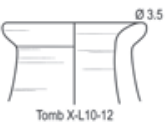

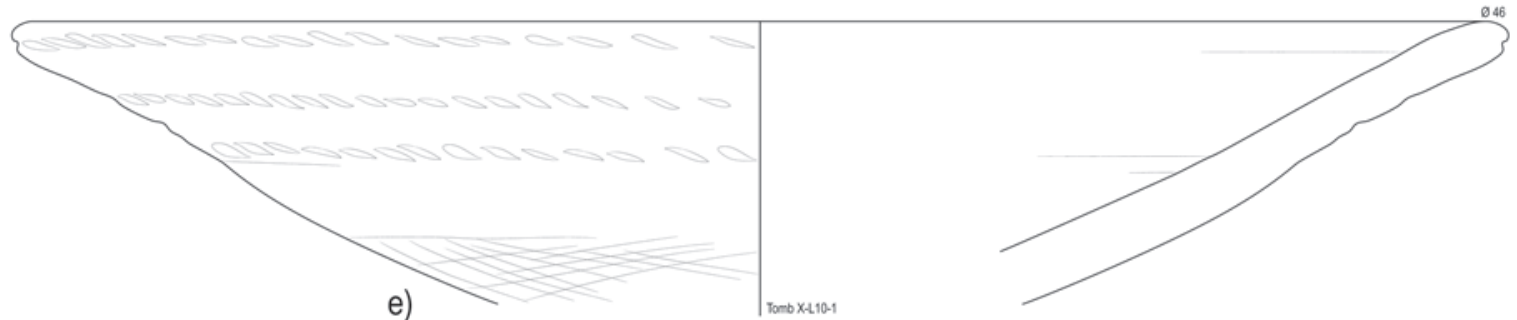

e)
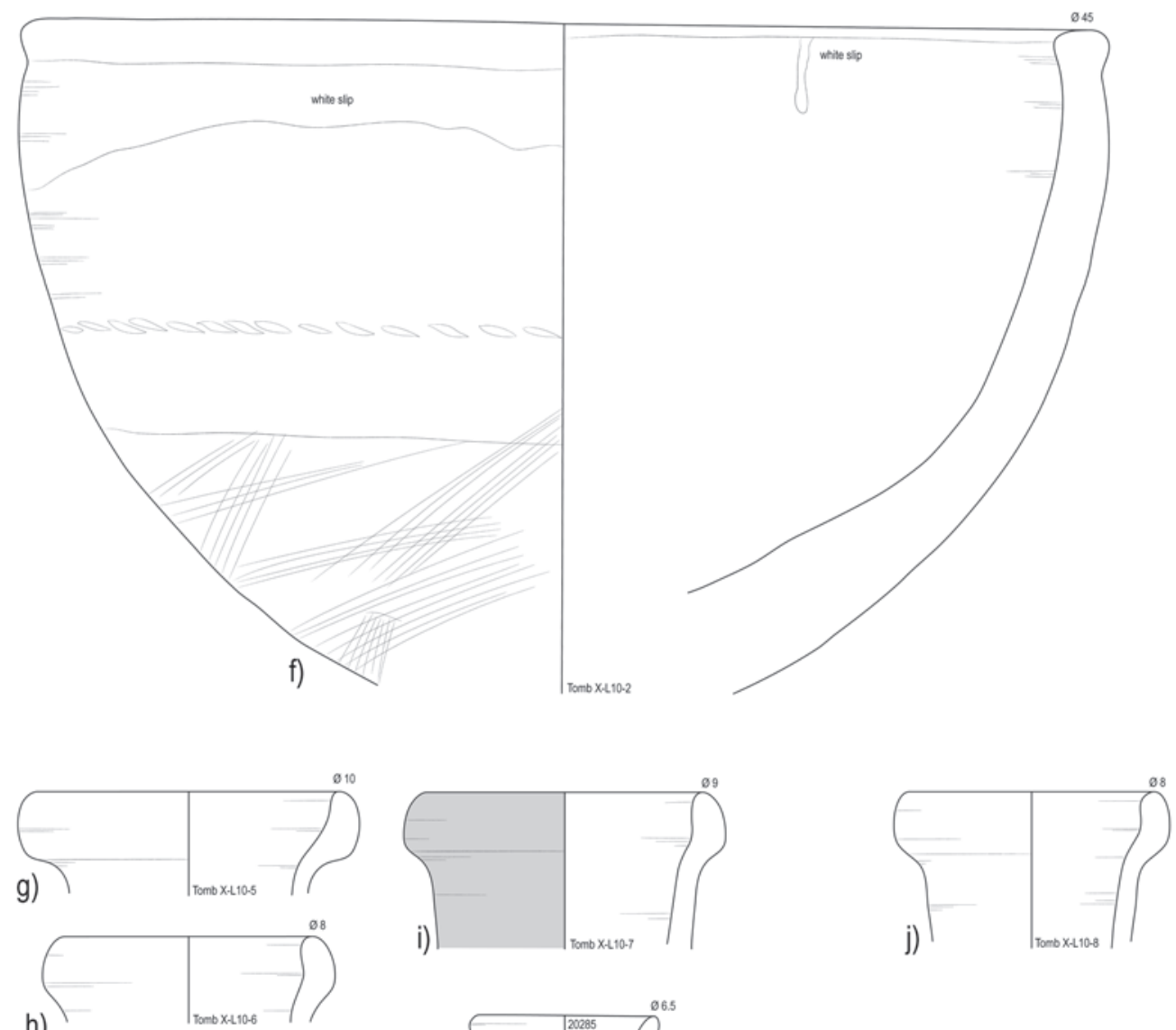

h)

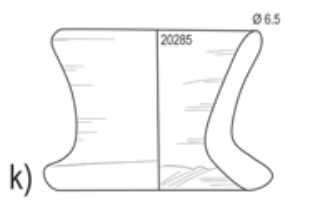

Fig. 29 a-j) Pottery from Tomb X-L10. k) Pottery from Tomb X-L11, late Middle and New (?) Kingdoms, drawn by B. Bader inked by E. Schuster. 
Tomb X-L10-7, rd.=9.0, pres.ht. $=5.4$, Nile C2, surface red stipped on exterior, soft.

Tomb X-L10-8, rd.= 8.0, pres.ht.= 5.2, Nile C2, surface eroded, soft.

Tomb X-L11 does not differ in essence from the previous contexts except for the presence of a ring stand with a direct rim, which seems to belong rather to the New Kingdom tradition than earlier. Beside this vessel (no. 20285, Fig. 29.k) no different types appeared: hemispherical cup fragments, model vessel fragments (dishes, bowls and jars, some in Nile B1), large Nile C2 dishes and basins, Nile $\mathrm{C} 2$ kettle rims of beer jars with diameters of $8.0-9.0 \mathrm{~cm}$. Again the dominant type in the body fragments were from Nile C2 'beer' jars and Nile C2 open shapes. The New Kingdom component was very small with two more possible body sherds (difficult to identify due to massive surface erosion).

No. 20285, rd. $=6.5$, bd. $=7.0$, ht. $=5.0$, Nile B2, section black core with light brown oxidation zones, surface uncoated (also eroded, 10YR7/3 very pale brown), wheel turned (W1), base scraped on interior, generally good quality.

Tomb X-L12 contained only Middle Kingdom material, again all the fragments were badly eroded. The repertoire found is congruent with a typical late Middle Kingdom tomb assemblage, of course the material was thrown about and smashed and some material might be missing.

\section{Nile B1 - Dish (Fig. 30.a)}

This small shallow dish made from Nile B1 is a more unusual addition to the corpus already existing. The manufacturing technology groups this vessel clearly with the Middle Kingdom tradition of pottery making. ${ }^{226}$

Tomb X-L12-5, rd.= 14.0, pres.ht.= 3.2, Nile B1, surface uncoated, coiled and turned, base scraped on exterior, interior well smoothed.

\section{Nile B1/B2 - Hemispherical cups (Fig. 30.b-c)}

The diagnostic fragments of hemispherical cups consist of both Nile B1 (base) and Nile B2, and the rims show an open shape with a rim diameter of $12.0 \mathrm{~cm}$. One of the rim fragments is uncoated whilst the other had a red rim band applied.
Tomb X-L12-2, rd.= 12.0, pres.ht.=4.1, Nile B2, surface uncoated (? eroded), coiled and turned.

Tomb X-L12-4, rd. $=12.0$ ?, pres.ht. $=6.3$, Nile B2, surface uncoated with red slipped rim band, coiled and turned.

\section{Nile B2 - Model dishes and restricted bowls (Fig. 30.d-f)}

A complete profile of a model dish came to light in L12. A model bowl such as no Tomb X-L12-3 with a very thin folded rim and a presumably round base has not been encountered in another tomb but L11 yielded such a fragment. They did not join. The technology of manufacture is in keeping with that of the late Middle Kingdom. The other small fragment might have belonged to a bowl/or jar of another type.

No. $8798, \mathrm{rd} .=8.3, \mathrm{bd} .=3.8, \mathrm{ht} .=2.8$, Nile B2 $/ 3$, section red core brown oxidation zones, surface uncoated (5YR6/6 reddish yellow), coiled and turned (W1?), base string cut.

Tomb X-L12-3, rd.=4.0, pres.ht.=4.3, Nile B2, surface red slipped on exterior and on rim interior, coiled and turned, base scraped on exterior.

Tomb X-L12-9, rd.=8.0, pres.ht.= 1.7, Nile B2 fine variant, surface red slipped on exterior and interior, coiled and turned (W1?).

\section{Nile B2 - Model jars (Fig. 30.g-h)}

The body of model jar no Tomb X-L12-6 resembles very much those of Tomb VI-L12-17 (Fig. $20 . m-n)$, whilst the other example, no. 8800 , has the shape of a canopic jar, which was discussed before (see Fig. 20.i, 25.c).

Tomb X-L12-6, pres.ht. $=8.1$, Nile B2, surface eroded, extremely soft, coiled and turned, and squeezed together as shown by folds on interior.

No. $8800, \mathrm{rd} .=3.8, \mathrm{bd} .=3.6, \mathrm{ht} .=6.1$, Nile B2/3, section not visible, intact, surface totally eroded, soft (5YR6/8 reddish yellow), coiled and turned (W1?), base string cut.

\section{Nile B2 - Jar with undulating contour (Fig. 30.i)}

This is the rare occurrence of a jar with undulating contour in the ceramic corpus of the tombs in the mortuary temple, which interestingly is absent from the publication of the ceramic material from Dra Abu el-Naga. It appears in the repertoire of

226 Larger examples are listed in the Middle Kingdom Handbook. SchiestL/Seiler 2012, 147. 


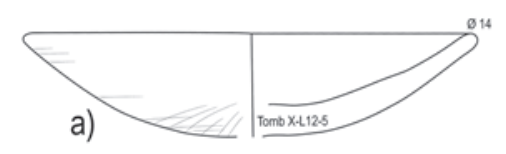

a)
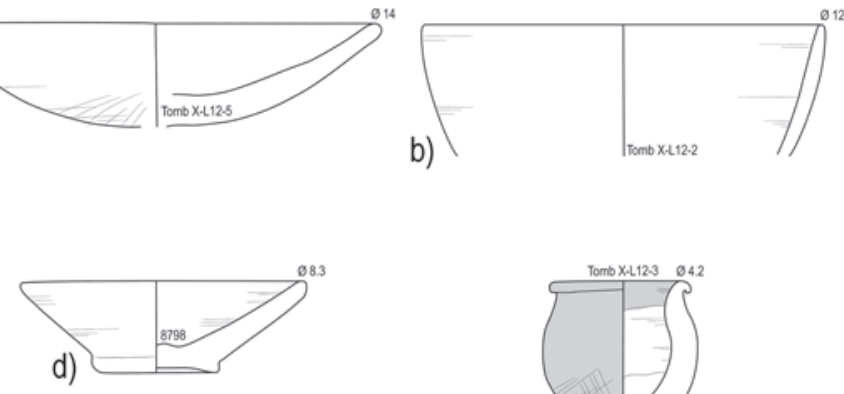

\begin{tabular}{c}
${ }^{10 \mathrm{~cm}}$ \\
-5 \\
\hline- \\
\hline- \\
\hline
\end{tabular}

g)
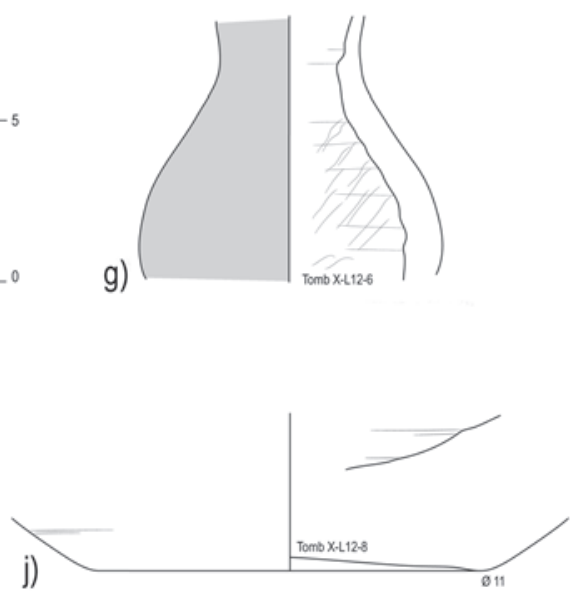
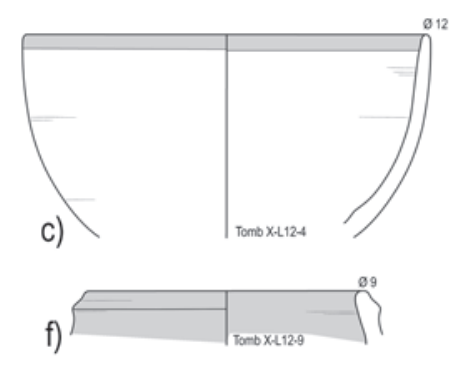

e)
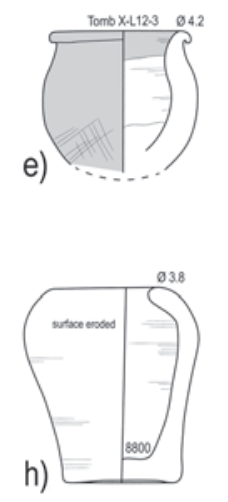

Fig. 30 Pottery from Tomb X-L12, late Middle Kingdom to Second Intermediate Period, drawn by B. Bader, inked by E. Schuster.

one of the tombs in the Ramesseum very similar to the current one. ${ }^{227}$

No. 8799 , rd. $=5.0$, pres.ht. $=9.1$, Nile B2, section uniform light brown, surface red slipped on top part on exterior (10R6/8 light red) and on rim interior, natural colour 7,5YR6/4 light brown), coiled and turned, body squeezed and slightly turned (folds on interior).

\section{Nile C2 - Basins and bowls (Fig. 30.j)}

The two base fragments of open shapes in Nile C2 represent again large dishes with rounded bases and basins with a flattened base. Only the latter will be shown, because the former is well represented in the previous figures (cf. Fig. 27.j, 28.c).

Tomb X-L12-8, bd.= 11.0, pres.ht. $=4.8$, Nile $\mathrm{C} 4$, surface uncoated, base trimmed with a knife.

\section{Nile C2 - 'Beer' jars (Fig. 30.k)}

The last beer jar rim fragment to be discussed from this tomb also shows the kettle rim, this time with a tubular neck although it does not seem to be overly long as it starts to flare out towards a shoul-

227 Nelson 2013, fig. 2.c.

228 SChiestr/Seiler 2012, 70-75, DAN 3. der. This is in keeping with the complete profile of 20263 from L9. ${ }^{228}$

Tomb X-L1, rd.=9.0, pres.ht. $=8.2$, Nile $\mathrm{C} 2$, surface eroded (uncoated?), coiled and turned.

\subsection{9.) Tomb XIV (square 980 -Sourth1) ${ }^{229}$}

The ceramic material from Tomb XIV, in which the high status burial of a lady with exquisite Middle Kingdom jewellery was found under a rock fall (L9), is extremely interesting although it has been scattered through the tomb chambers and the shaft. It was excavated by I. Macias in 2014. Reconstruction of the material is still in progress therefore only a preview of the ceramic repertoire is given here.

The tomb consisted of a vertical shaft with two chambers and several niches. The Layers 1 to 6 represent shaft fill, whilst the others (7 to 11) came from the chambers. The work on the reconstruction of the ceramic vessels proved that the broken material was distributed through most of the layers as vessels joined across them: L3/L4/L7/L11,

229 Seco Álvarez/Martinez Babon 2015b, 39-41. 
L4/L6，L6/L7/L8/L10/L11，L3/L7/L8，L7/L8，L7/ L8/L9, L7/L8/L10, L8/L10, L3/L4/L7/L8/L11. Thus, it can be stated that post-burial activities took place within this tomb that distributed broken material across the whole tomb. Interestingly New Kingdom intrusions (mainly some body sherds) were found in the shaft fill and the contexts immediately adjacent to the shaft but not with the human remains of the lady and jewellery and not in the layers further away from the shaft. From this we can conclude that the initial destruction took place quite soon after the burial because the other ceramic material seems quite homogeneous to date and does not include any later Second Intermediate Period material, but further research is necessary to prove this first impression.

Because the cross joins connect all the layers the following preliminary description concentrates on vessel types and not on context.

The ceramic corpus of this tomb includes a larger number of fine Nile B1 and Nile B2 hemispherical cups (subject to more restoration work), very well made Nile B1/2 medium sized dishes with dark red slip, a wide range of model vessels mostly dishes (cf. e. g. Fig. 10.d-s, 11.a-g), model jars (cf. Fig. 11.h, Fig. 15.f), and 'canopic' shapes (cf. Fig. 25.c, 30.h) and a few examples of small finely made restricted bowls with short modelled $\mathrm{rim}^{230}$. Some examples were equipped with red rims and vertical striped decoration. Several Nile B2 jars with undulating contour and flat base cut with a tool ${ }^{231}$ and fine jars with funnel necks ${ }^{232}$ were also found. The preliminary Nile B corpus is rounded off with a single small lid with a painted red cross on the exterior and pre-firing hole pinched into the top of it.

The Nile C2 corpus is represented by several large shallow dishes with and without wide red slipped rim bands (cf. Figs. 21.b, f-g, 27.j, 28.c) and a deep basin (no. 20114, Fig. 31.a).

No. 20114 (L4 and L6), rd.= 36.0, ht.= 20.5, Nile $\mathrm{C} 2$, section red core, brown oxidation zones, uncoated surface (10YR7/3-4 very pale brown) with wide irregular white slipped rim band on exterior (5Y8/1 white) and thin rim band on interior, coiled and turned, base roughly trimmed, rim trimmed with tool only on one side of vessel, three

\footnotetext{
230 Schiestl/Seiler 2012, 343, no 1.

231 Schiestl/Seiler 2012, 696-697, type II.L.b.

232 SEILER 2005, Falttafel 8.1-2.
}

rows of rope marks on exterior, well smoothed on interior.

A very interesting find are two compartmented bowls with seven compartments and a central cup made from Nile C2 with a white slip (nos 20283 and 20284) very similar to that found in the tomb of Senebtisi at Lisht. ${ }^{233}$ Their surfaces, especially at the base are very eroded. It remains unclear if this erosion is derived from heavy use (unclear which use, as no traces of contents were left) or throwing about in the tomb or flood water in the tomb. Other Nile C2 vessel types were a ring stand base and several beer jars. Two were already reconstructed to complete profiles and most of the rim fragments seem to belong to the 'kettle' type, but the final analysis must show if other rim types also occur in the sherd material. At least eight beer jars belonged to the burial and the body shape seems to be mostly ovoid.

Also at least five conical vessels (cf. Fig. 14.f, 18.j) made of Marl clay fabrics were found (also in L9, together with the body and the jewellery), suggesting very convincingly that this type, indeed, also belongs to the late Middle Kingdom. They vary in height and surface treatment: some were red slipped and burnished ${ }^{234}$ whilst others were left uncoated. The manufacturing technology is in keeping with the examples already mentioned.

The handle of an imported Canaanite jar from L6 belongs rather to the Late Bronze Age than the Middle Bronze Age, because the fabric does not resemble those known from other sites in the earlier period.

An interesting contrast to Seiler's older Middle Kingdom phase is provided by the total lack of Marl C1 storage jars. ${ }^{235}$ Hitherto only one storage jar rim fragment was found in a surface context and a few body fragments of the same type in other (surface) contexts but not more than 20 altogether.

The dating of the material (except for the small amount of intrusive New Kingdom material in Layers 2, 3, 6, 7 and 11) is clearly late MK possibly to be equated with the late $12^{\text {th }}$ or early $13^{\text {th }}$ Dynasty. It seems likely that the later end of this phase should be preferred but currently this cannot yet be proven. The intrusion of the New Kingdom

\footnotetext{
233 Mace/Winlock 1916, 112-113, pl. XXXVA-B. Now in the Metropolitan Museum in New York (09.180.891), where Bettina Bader saw it in the permanent exhibition.

234 Cf. Seco Álvarez et al. in press, ms 21, fig. 26.h.

235 SeILER 2005, 76-77.
} 

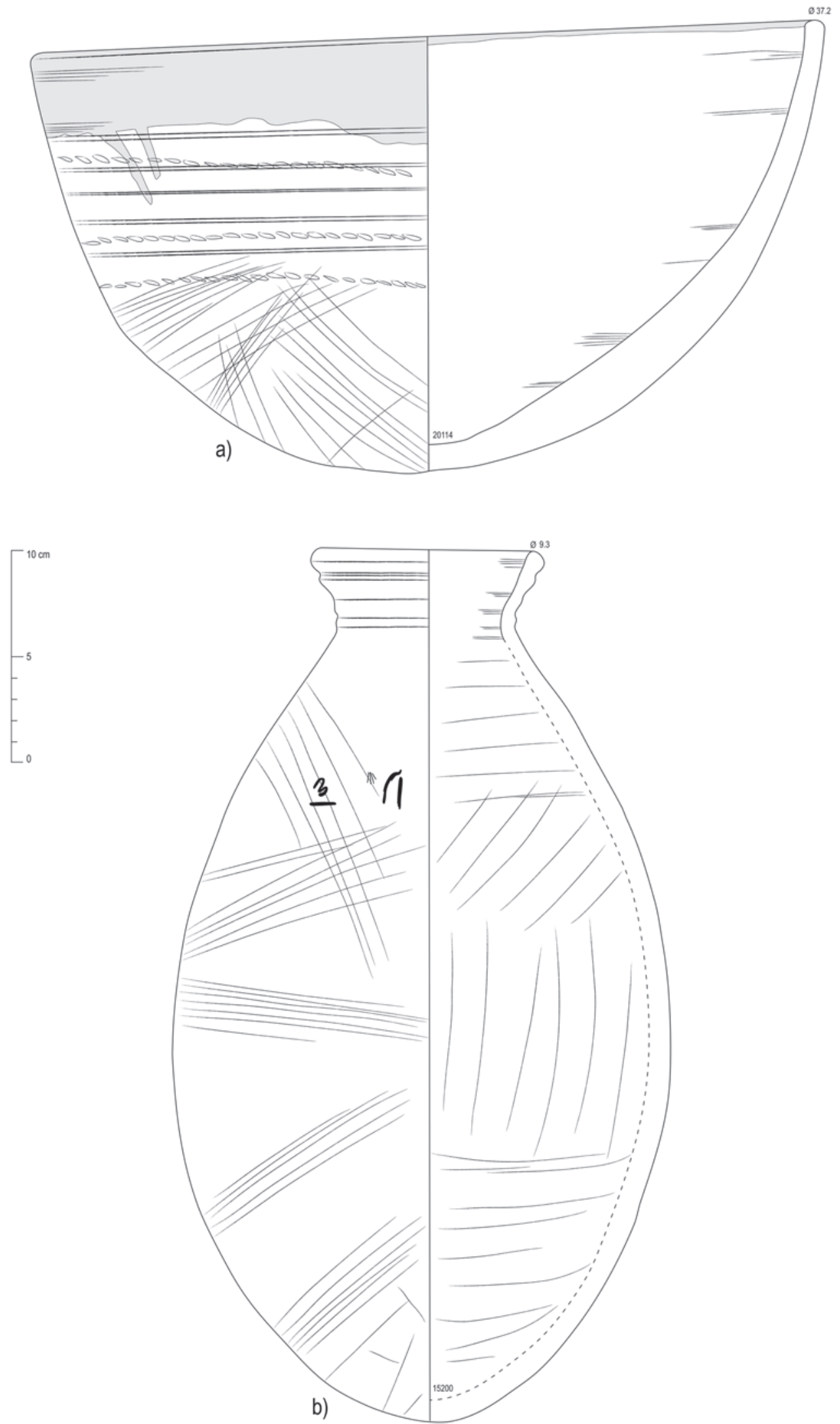

Fig. 31 a) Pottery from Tomb XIV. b) Pottery from Tomb XV, late Middle Kingdom, drawn by B. Bader, inked by E. Schuster. 
material can be suspected during the building process of the temple of millions of years of Thutmosis III.

\subsubsection{0.) Tomb XV (square 970-South 2) 236}

Tomb XV was also excavated in 2014 by I. Macias. It seems to have been used in the Middle Kingdom as well, most probably in the late $12^{\text {th }}$ Dynasty as an almost intact Marl C, compact vessel of ovoid shape with corrugated neck was found in the shaft fill (see Fig. 31.b). ${ }^{237}$

No. 15200 (L8), rd.= 9.3, max.d. $=23.3$, ht. $=$ 40.7, Marl C, compact, section uniform red, surface covered with thick yellowish scum (2.5Y8/2), handmade with rim turned on device, base handmade and scraped on exterior, very well smoothed. Part of a hieratic inscription in top third of vessel; ${ }^{238}$ vessel was covered additionally with white powder, which is lime confirmed by lemon test (frothing was observed), almost complete, weight $3.3 \mathrm{~kg}$.

The surface layer L1 contained in addition to the usual Middle Kingdom and New Kingdom mix also a modern Ballâs jar. The shaft was filled with Layers L2, L5, L6, L7, L9 and L10 whilst the chamber/niche contained layers L8 and L11. New Kingdom material of the earlier part of the $18^{\text {th }}$ Dynasty was found in L5, L6, L7 and L8 but not in other parts of the tomb. Some of the vessels could be reconstructed such as a quite large 'BB jar' and a Marl A4 bowl with wheelmade ring base.

Cross joins were found hitherto between layers L5/L6, L7/L8/L10/L11, L8/L9, L9/L10 and L8/L11. As the reconstruction work is not finalised yet, there may be more joins yet to come.

The Nile B1 and B2 pottery repertoire as far as it is known to date comprises small and medium plates/dishes of very good quality, at least one small dish with an inturned lip and a red slip on interior and exterior, a considerable number of hemispherical cups, which need much restoration work, a Nile B2/C1 dish with undulating rim and a restricted bowl with short spout and finger pinched base ${ }^{239}$ but with thickened rim, red slip on exterior down two thirds of the vessel height. The model vessels include dishes and bowls as well at least one example with an in-curved rim. The closed vessels comprise medium ovoid jars with a funnel neck, ${ }^{240}$ a globular jar with incised decoration of zig zag and horizontal lines red slipped in the upper part of the vessel, medium jars with a vertical neck, several red burnished bottles but not necessarily with high funnel neck and several jars with undulating contour and flat base with the top part red slipped.

A vessel without comparanda in Thebes is a thin walled footed bowl made from Marl clay or a

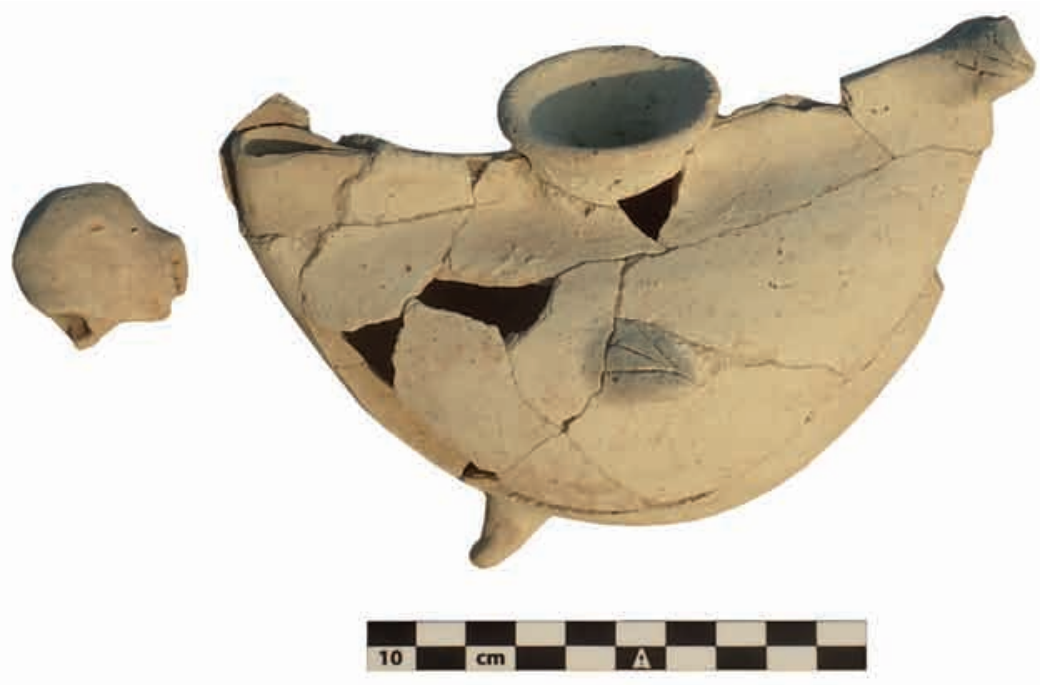

Fig. 32 Pottery from Tomb XV, probably Second Intermediate Period, photo by B. Bader.

236 Seco Álvarez/Martinez Babon $2015 b$.

237 BADER 2001, 129-145; ARNOLD 1981.

238 Dd mdw[?] ....hk3 t 7[?]....would be the equivalent 33.25 litres. Cf. BADER 2011, 159-160 for other inscriptions on such pottery.
239 Similar to Schiestl/Seiler 2012, 344, no 10-11, mostly from the $12^{\text {th }}$ Dynasty.

240 SeILer 2005, Falttafel 8.1-2. 
white slipped Nile clay fabric with the foot closed at the bottom. ${ }^{241}$ A Nile B2 ring stand of very good quality rounds off the Nile B repertoire.

Ubiquitous among the pottery types of this tomb are Nile C2 'beer' jars with folded rims as well as kettle rims and straight tubular necks that are not very long. One example shows a sharply defined edge on the exterior lip but none that could be reconstructed to a full profile. An interesting type that had not appeared before was made from Nile C2 with a restricted tendency and folded rims. A red slip had been applied on the interior and exterior, perhaps they should be reconstructed to bag-shaped beakers, ${ }^{242}$ or a carinated form. ${ }^{243}$

In this tomb the Marl clay fabric component is more prominent than in the others. Beside a Marl A ringstand, there were several small to medium jars with either globular or bag shaped contour. The globular one had a narrow neck with a very finely corrugated neck. Again the Marl A4 conical vessels appeared either red slipped and burnished on the exterior or uncoated. Especially noteworthy is the figurative vessel in Marl A4 in the shape of a bird, which may hint at a later Second Intermediate Period date for this phase ${ }^{244}$ (no. 15201, L8 and L10, Fig. 32).

\subsubsection{1.) Tomb XVI (square 980-South 2)}

Tomb XVI was excavated by A. Moreno Onorato in 2014 and it was totally destroyed by the building project of Thutmosis III. Only a few depositions of smaller pottery vessels were preserved in layers L2, L3 and L10 that were close to the bedrock floor representing the remains of a corridor and a burial chamber. The vertical preservation of the architecture was below $0.5 \mathrm{~m}$ so that the Middle Kingdom material was mixed with New Kingdom pottery from the actual building process. Only the peculiarities of the corpora date the activities.

One very fine round based carinated cup made of Marl A (no. 8789) was reconstructed from sherds found in all of those layers. All in all the pottery from the burial, which can be reconstructed, gives the impression that it belongs to the earlier part of the Middle Kingdom. The quality of the

\footnotetext{
241 See BADER 2001, 60, Type 17 for the form.

242 SChiestl/Seiler 2012, 545

243 MACE/WINLOCK 1916, fig. 82.16.

244 SEILER 2010, fig. 14. ,17 $7^{\text {th }}$ Dynastyc.

245 SCHiestl/Seiler 2012, 296-297.
}

pottery is excellent and the repertoire ranges from fine hemispherical cups (one of them, no. 8797, with a very large ancient hole in the base), to several very well made round based model dishes, a model jar and models of hemispherical cups as well as an elongated pointed jar. One model cup is shown here (Fig. 33.a).

No. 8782 , rd. $=7.5$, bd. $=3.8$, ht. $=4.7$, Nile B2, section uniform light brown (7.5YR5/4 brown), surface uncoated with red slipped rim on exterior and interior, some red painted spots on the exterior (accidental?), good quality of manufacture, coiled and turned (W1?), base string cut.

Among the shapes found was the base of a chalice from fine Nile B1 with a dark red slip. This type is common in the early Middle Kingdom around the beginning of the $12^{\text {th }}$ Dynasty in other parts of Egypt. ${ }^{245}$ More analytic and comparative work has to be done to ascertain the date range of this deposit.

\subsubsection{2.) Summary of tombs within the temple area}

Although the study of the material is not finished for all the tombs ${ }^{246}$ within the area of the temple of Thutmosis III, a summary can be offered.

Interestingly not one trace of the earlier material from the tomb cluster north of the temple enclosure wall was hitherto found within the temple area (cf. 5.1), hence this phase may be termed (Phase 1).

The ceramic material suggests that already in the early $12^{\text {th }}$ Dynasty this area was used for burials (Phase 2). It is clear from the architectural features (corridors, shafts, chambers) that they were built for affluent people probably belonging to a local élite. It is also interesting that two of the tombs with the earliest pottery possess corridors (Tomb III, IV), whilst those with later pottery are shaft tombs. Also the tombs are spatially quite close to other early $12^{\text {th }}$ Dynasty tombs such as at least one phase of Tomb ' 79 beneath the mortuary temple of Thutmosis IV, ${ }^{247}$ one tomb (A17) in the area of the mortuary temple of Amenhotep II $^{248}$ and others located behind the hill of Sheikh abd el-Gurna discussed by Dorothea Arnold. ${ }^{249}$ It is also noteable that these earlier tombs all contained

\footnotetext{
246 Tombs XIV, XV and XVI still need to be studied in detail.

247 Guidotti 1985. Measuring on google maps resulted in an approximate distance of $570 \mathrm{~m}$.

248 Consonni/Sesana 2016.

249 ARNOLD 1991.
} 


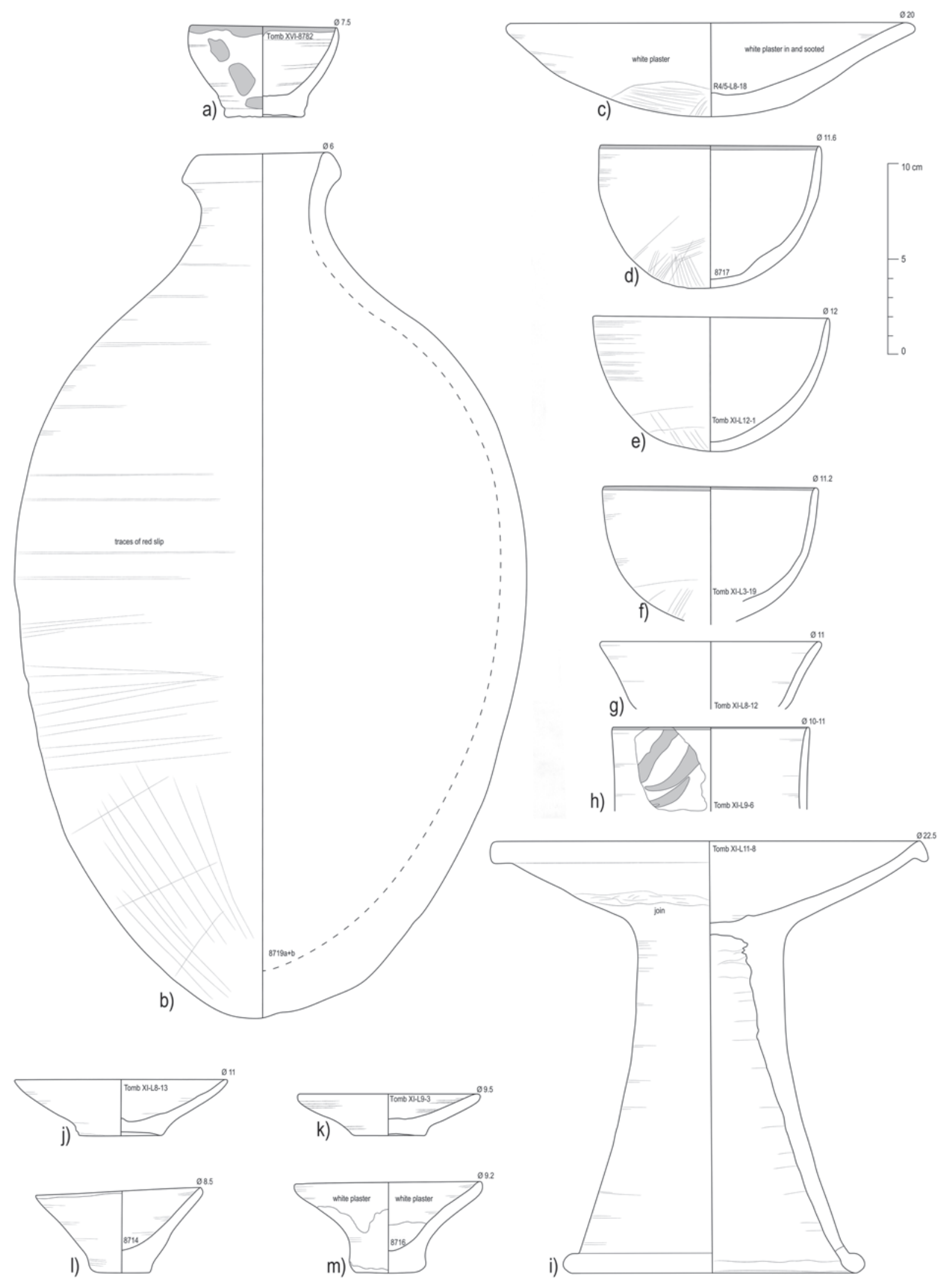

Fig. 33 a) Pottery from Tomb XVI. b-1) Pottery from Tomb XI, late Middle Kingdom, drawn by B. Bader, inked by E. Schuster. 
pottery offering tables. ${ }^{250}$ It is possible that these seemingly separate tomb clusters belong to a larger cemetery.

It is difficult to state when these early $12^{\text {th }}$ Dynasty tombs were robbed and/or reused and new tombs built, but there is clearly a third phase (Phase 3) dateable to the 'late $12^{\text {th }}$ and/or $13^{\text {th }}$ Dynasty', ceramic remnants of which were found in almost all the tombs listed (II, III, IV, V, VI, VII, IX, X, XIV and XV). It must be stated in this instance that the length of the phases proposed cannot be estimated with any certainty at the moment. Further, it is currently not possible to calculate a plausible time span for how long into the ' $13^{\text {th }}$ Dynasty' and beyond this third Phase would last. Most probably this phase continues into the early Second Intermediate Period. ${ }^{251}$

Furthermore, there are some hints for yet another phase of use of these tombs (Phase 4) some time in the later Second Intermediate Period (or ' $17^{\text {th }}$ Dynasty'), but very little remains from this period (e. g. burial of 4 individuals in Tomb III with one pottery vessel ${ }^{252}$ and perhaps in Tomb V, see above).

Finally the destruction of some of the tombs during the construction of Thutmosis' III temple of millions of years can be regarded as Phase 5 .

\section{6.) The Middle Kingdom tomb north of the enclosure wall - Tomb XI and its use life}

Immediately north and south of the enclosure walls of the temple several more tombs were found (VIII, ${ }^{253}$ XVII) but only XI will be treated here, because work on the other tombs is not far enough advanced to give a proper overview.

\section{1.) Tomb XI (square R4/5) ${ }^{254}$}

This tomb was excavated in 2013 by M. Seco. It has a deep shaft of $7.0 \mathrm{~m}$ and two chambers one in the north and one in the south: Room 1 and Room 2 as well as another chamber (Room 3) containing a square pillar in the middle.

Layer R4/5-L2 contained an enormous amount of pottery from the surface above the mouth of the tomb shaft. The major component of this assem-

\footnotetext{
250 Guidotti 1985, fig. 10; Consonni/Sesana 2016, fig. 5; Bader in Seco Álvarez et al. 2012/13 (appeared 2016), 362, fig. 35.

251 Termed by Seiler ' $16^{\text {th }}$ Dynasty'.

252 Seco Álvarez et al. 2012/13 (appeared 2016), 337-338.
}

blage was fragmentary New Kingdom 'BB jars' (see below). R4/5-L3 is from the top of the shaft, whilst L6, L7 and L8 represent shaft fill. L8 is the last layer before the floor of the bedrock was reached (with a 'modern' cooking tagine very much smoke blackened; see Fig. 39.b) indicating severe disturbance of the contents of the tomb. R4/5-L9 represents the chamber in the north, Room 1, whilst R4/5-L10 signifies the pit for the sarcophagus in Room 1. There a Middle Kingdom carinated miniature cup with vertical red stripes was found, some New Kingdom material but also a much later qulla with a filter neck (see below). The sloping passage to Room 2 was termed L11, the fill of Room 2, the chamber in the south, L12, the niche L13 and L14 represents the fill of Room 3.

The ceramic finds from this tomb are very heterogeneous with elements from the early and later Middle Kingdom, the early $18^{\text {th }}$ Dynasty, the later New Kingdom, the $25^{\text {th }}$ Dynasty, an even later phase in the $7^{\text {th }}$ to $4^{\text {th }}$ century B.C. and some fragments that may be classed as modern. The Middle and New Kingdoms are best represented whilst the others often only appear as single sherds or vessels. Thus, the occupational history of this tomb differs from those inside the temple mentioned before. The main reason for this difference can be seen in the position of the tomb because outside of the temple area proper it was more exposed to external influences such as frequent entering and/ or local activity. The ceramic evidence attests to a much wider chronological range of activity and use periods although it remains unclear which activity exactly took place.

Joins between vessels were noted from L2/L3, L8/L13, L10/L13, L10/L11, L12/14 and L13/14. Due to the mixed nature of all the Layers the pottery is given in chronological order from the oldest to latest and not in contextual groups. Preference is given here to complete profiles and fragments of ceramic vessels not yet discussed.

\subsection{1.) The Middle Kingdom pottery corpus}

One of the most striking new shapes appearing in Tomb XI is an early-mid $12^{\text {th }}$ Dynasty 'beer' jar

\footnotetext{
253 Seco Álvarez et al. in press, plan 2, fig. 4; excavated by M. Seco in 2013.

254 Seco Álvarez 2015, 122-123, plan 4; Seco Álvarez/MarTINEZ BABON 2015c.
} 
with ellipsoid body contour and a short narrow neck with folded lip, which also has parallels in the Theban necropolis ${ }^{255}$ (Fig. 33.b).

No. 8719 (L3), rd.=6.3, max.d. $=26.3$, ht. $=46.0$, Nile C2, section not visible intact, surface eroded traces of red slip on exterior (10 R 5/6 red), coiled and turned, base scraped on the exterior, intact, weight $5.75 \mathrm{~kg}$.

Whilst this one particular vessel sticks out as being earlier than most, the other vessels and fragments can only be assigned to a general late(r) Middle Kingdom horizon (late $12^{\text {th }} / 13^{\text {th }}$ Dynasty perhaps into the early Second Intermediate Period. The corpus of the Middle Kingdom encompasses many of those shapes that were already discussed with the tombs above, also among the types were jars with undulating contour and dishes with white dots.

Nile B1 and B2 - Hemispherical cups (Fig. 33.d-f)

All of these cups encountered in Tomb XI (L2, L3, L11, L8, L9) show an open shape except one with straight walls and all were decorated with a red slipped rim band on the interior and the exterior. The rim diameter ranges from $11.0 \mathrm{~cm}$ to $13.5 \mathrm{~cm}$. Three examples were preserved with a full profile but they are not uniform. ${ }^{256}$

No. 8717 (L3), rd.=11.2, max.d.=11.6, ht.=7.5, Nile B1, section uniform reddish brown, surface uncoated (2.5 YR 6/4 weak red) with thin red rim band on interior and exterior (10R6/6 light red), coiled and turned W1?), base trimmed (in a different pattern to Memphis), VI 154.7, almost complete, weight $250 \mathrm{~g}$.

$\mathrm{R} 4 / 5-\mathrm{L} 12-1, \mathrm{rd} .=12.0$, max.d. $=12.3$, ht. $=7.1$, Nile B1, surface sooted and blackened on interior and exterior, coiled and turned, base trimmed, VI 172.3 .

$\mathrm{R} 4 / 5-\mathrm{L} 3-19, \mathrm{rd} .=10.8$, max.d. $=11.2$, pres.ht. $=$ 7.1, Nile B1, surface uncoated with thin red rim band on interior and exterior, coiled and turned, base trimmed.

\section{Nile B2/C1 - Medium shallow dish (Fig. 33.c)}

This vessel cannot be dated more precisely than a general Middle Kingdom date. ${ }^{257}$

\footnotetext{
255 Schiestl/Seiler 2012, 646-647.

256 Schiestl/Seiler 2012, 96-98, 100-104.

257 Schiestl/SEILER 2012, 147.

258 SEILER 2010, fig. 13.
}

No. R4/5-L8-18, rd.=20.0, ht. $=4.9$, Nile B2/C1, fabric beige and soft, surface burnt and sooted and covered with white plaster on interior and exterior, coiled and turned, base scraped.

\section{Nile B1 - Carinated cups (Fig. 33.g-h)}

Two very fine and well-made carinated cups with the carination just preserved were found. This type group was already discussed (see Fig. 12.f, 14.a).

R4/5-L8-12, rd. $=11$, pres.ht. $=3.5$, Nile B1, surface uncoated, coiled and turned (W1?).

R4/5-L9-6, rd.= 10-11.0, pres.ht.=4.3, Nile B1, surface uncoated with red slipped rim band on interior and exterior

\section{Nile B2/C1 - Offering stand (Fig. 33.i)}

This type of offering stand is made from a fairly fine fabric with folded rim on base and rim and a wide open dish. The feet are only moderately high. No exact parallel is represented in the Middle Kingdom pottery Handbook but appears at Dra Abu el-Naga dated to the ' $17^{\text {th }}$ Dynasty. ${ }^{258}$

R4/5-L11-8, rd. $=15$, bd. $=15.0$, ht. $=22.60$ (reconstructed from non-joining sherds), Nile B2, surface uncoated, coiled and turned, base turned.

\section{Various fabrics - Model vessels (Fig. 33.j-m,} 34.a-c)

A large amount of small dishes and bowls was found in this tomb, but because it was quite disturbed only a small selection of different types of flat based shallow and deeper dishes (already discussed), carinated cups and restricted vessels will be presented here. No. 8716 (Fig. 33.m) may represent a model footed bowl, which is not represented in the Middle Kingdom Handbook. It may perhaps date later. In contrast the carinated cup (Fig. 34.a) is clearly a Middle Kingdom type ${ }^{259}$ represented in the Theban necropolis ${ }^{260}$ but without the red painted decoration. The round based cup no. 8715 also has no exact parallels in terms of the manufacturing technology in the Handbook. ${ }^{261}$ Finally, the restricted cup no. 8718 finds a general parallel. ${ }^{262}$

R4/5-L8-13, rd.= 11.0, bd.=4.4, ht.=3.0, Nile B3, surface uncoated, wheelmade (W1?), base string cut.

\footnotetext{
259 Aston 2004b, vol. 2, pl. 1.2.

260 SChiestl/Seiler 2012, 912-913

261 Schiestl/Seiler 2012, 850-851.

262 Schiestl/Seiler 2012, 1004-1005.
} 
a)
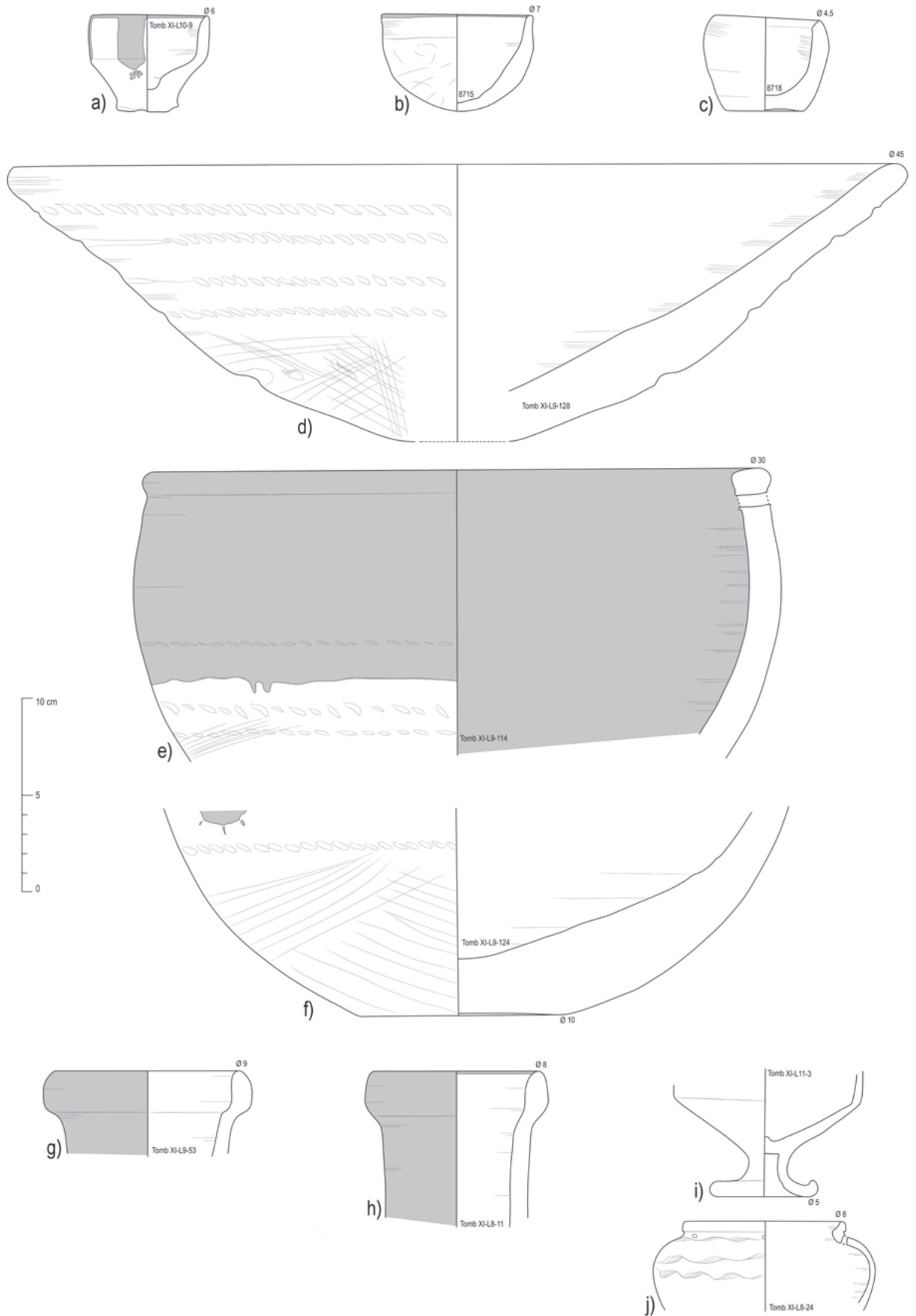

Fig. 34 Pottery from Tomb XI, Middle Kingdom, drawn by B. Bader, inked by E. Schuster. 
R4/5-L9-3, rd. = 9.5, bd.= 3.7, ht.= 2.1, Nile B2, surface uncoated (7.5YR6/4 light brown), wheelmade (W1?), base string cut.

No. 8714 (L2), rd. $=8.5$, bd. $=8.5$, ht. $=4.0-4.7$, Nile B2, section uniform brown, surface uncoated (5YR5/4 reddish brown), wheelmade (W1?), base string cut.

No. 8716 (L3), rd.=9.2, bd.=9.6, max.d.=3.54.0 , ht. $=4.7$, Nile B2, section not visible intact, surface uncoated (10YR7/3 very pale brown) with white wide rim band (or mortar/plaster? 2.5Y8/2 pale yellow), wheelmade (W1), base string cut.

R4/5-L10-9, rd. $=6.0$, bd. $=3.2$, ht. $=5.0$, Nile B1, surface uncoated with red rim band, and vertical broad bands down to carination, coiled and turned, base string cut.

No. 8715 (L8), rd.=6.5-7.0, max.d.= 7.8, ht.= 5.0, Nile B2, section uniform light brown, surface uncoated (7.5YR6/4 light brown) with red rim band (10R5/6 red), combination technique of hand and wheelmade, base handmade and trimmed, uneven.

No. 8718 (L14), rd.=4.5, max.d.=6.5, bd. $=4.0$ 4.5 , ht. $=4.9$, Nile B2, section uniform light brown, uncoated (10YR6/3 pale brown), wheelmade (W1), base string cut.

\section{Nile C2 - Large dishes and bowls (Fig. 34.d)}

Several examples of large dishes were found in the tomb. Variations are with white cross, with white finger dots, with radial finger strokes in white paint and uncoated. The rim diameters range from 26.0 to $45.0 \mathrm{~cm}$. Several rows of string impressions are the norm. Other variations of large bowls include those with trimmed rims and open shape (cf. Fig. 12.h) in L9-101, L9-122. This group has already been discussed (p. 186).

$\mathrm{R} 4 / 5-\mathrm{L} 9-128, \mathrm{rd} .=45.0$, pres.ht. $=14.4$, Nile C2, surface uncoated (7.5YR6/4 light reddish brown), coiled and turned, base scraped on exterior, 4 rows of string impressions, smoothed on interior.

\section{Nile C2 - Large basins (Fig. 34.e-f)}

A larger number of fragments of basins with restricted rim with folded lips and pre-firing holes were found in several layers. They belong to at least two vessels as two complete flattened bases were found but could not be joined to rims. This variation of basins with flat base is not represented in the Middle Kingdom Handbook (only round and ring bases ${ }^{263}$ ), although there can be no doubt

\footnotetext{
263 Schiestl/Seiler 2012, 304-309, 314-325.

264 SEILER 2005, 70-75, 127-130.
}

that that the type belongs to the Middle Kingdom. Therefore, no chronological difference can be ascertained as yet.

R4/5-L9-114, $\mathrm{rd} .=30.0$, pres.ht. $=14.8$, Nile C2 + some lime, surface red slipped on interior and exterior below max.d., coiled and turned, base scraped, three rows of rope impressions, under the rim pre-fired hole made from outside to the inside.

R4/5-L9-124, bd. $=10.0$, pres.ht. $=10.7$, Nile C2, coiled and turned, base roughly scraped.

\section{Nile C2 - 'Beer' jars (Fig. 34.g-h)}

Apart from the complete example shown above (no. 8719) only a few rim fragments were found. The type (Seiler's DAN 2 or $3^{264}$ ) suggests a date in the later $13^{\text {th }}$ Dynasty or early Second Intermediate Period. One larger body fragment from L3 may be of ellipsoid or ovoid body shape but it is not well enough preserved to be certain. Also in the body sherd statistics the type is not well represented in Tomb XI: there were only 61 fragments with a weight of $7.5 \mathrm{~kg}$ from all layers. This is in contrast to Tombs V, VI, IX and X, and suggests thorough disturbance.

R4/5-L9-53, rd.=9.0, pres.ht.= 4.4, Nile C2, surface red slipped on exterior and interior on rim, coiled and turned.

R4/5-L8-11, rd. $=8.0$, pres.ht. $=7.9$, Nile C3, surface red slipped on exterior and interior on rim, coiled and turned.

\section{Marl A3 - Chalice (Fig. 34.i)}

Several fragments of very finely made chalices were found in this tomb. Their scarcity in publications leaves some doubt whether this material is modern or not. Only in the Ramesseum was similar pottery found and dated to the $17^{\text {th }}$ Dynasty. ${ }^{265}$

\section{Marl A4 - Conical vessels (not shown)}

Two examples of this type also appear in this tomb (cf. Fig. 14.f, 18.j) in L9-70-71 (see previous discussions).

$\mathrm{R} 4 / 5-\mathrm{L} 11-3, \mathrm{bd} .=5.0$, pres.ht. $=6.7$, Marl A3? very fine, surface uncoated, wheelmade, base wheelmade.

\section{Marl A4 - Squat jar with wavy decoration (Fig. 34.j)}

A very well-known type ${ }^{266}$ is represented by this high quality shouldered jar. Only the top part is

\footnotetext{
265 Nelson 2013, 93-97, fig. 2n-o.

266 Schiestl/Seiler 2012, 454-455, 11th to early $12^{\text {th }}$ Dynasty.
} 
preserved with a modelled rim to hold a lid. The shoulder was pierced pre-firing. This is the second pottery type with clear early dating beside the beer jar no. 8719.

R4/5-L8-24, rd.= 8.0, pres.ht.= 4.6, Marl A4, surface uncoated, pre-firing holes pierced from outside to inside, wheelmade, pre-firing incised decoration made with comb, surface very well smoothed.

\section{Nile C3 - Rectangular tray with square com- partments (Fig. 35)}

A particularly interesting item in the pottery corpus hitherto not very well known at all is a compartmented tray with at least 12 compartments as the joining fragments prove (Fig. 35). The pottery fabric was a rough Nile $\mathrm{C}$ clay covered in a thick white 'slip' which turned out to be a 'powder' or 'pigment' containing much lime as it reacted violently to the acid of a lemon. Similar trays but with fewer or more compartments were found at Dra Abu el Naga, ${ }^{267}$ Asasif ${ }^{268}$ and Tarif. ${ }^{269}$ Seiler interpreted the Dra Abu el-Naga example with four compartments as bread mould because remains of the contents seem to have been present and that vessel conformed to the usual appearance of bread moulds but did not show the thick white slip/covering. For this reason the current example does not seem to represent a bread mould.

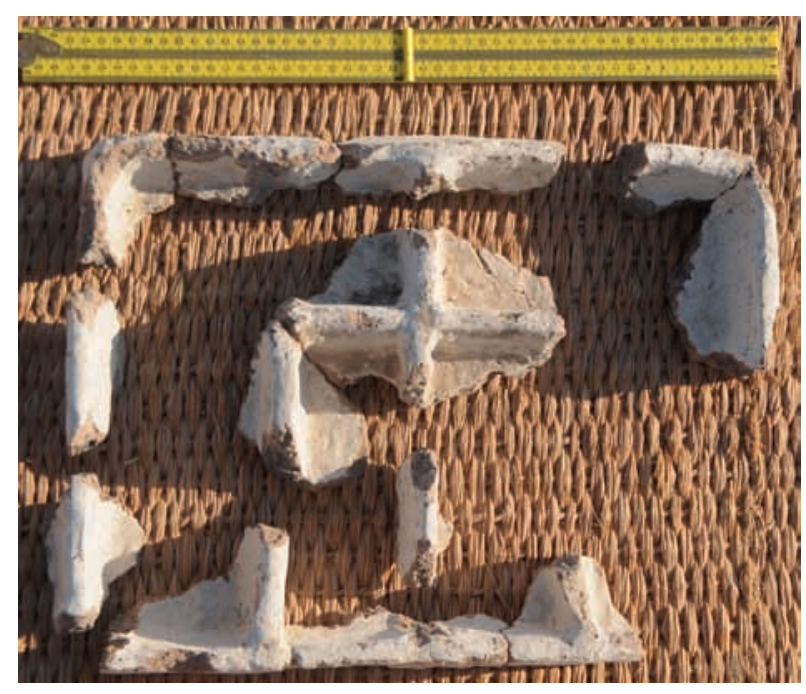

Fig. 35 Pottery from Tomb XI, Middle Kingdom, photo by B. Bader.

\footnotetext{
267 SeILER 2005, 68-69.

268 Carnarvon/Carter 1912, 51-54, tomb 24, pl.47.2 very elaborate with more compartments now in Cairo Museum.

269 Petrie 1909, plate XX.597.

270 Seco Álvarez 2015, 109-110
}

\subsection{2.) The New Kingdom pottery corpus}

The New Kingdom pottery corpus found in and around this tomb was marked above all by the massive dumping of ceramic material to the north of the northern enclosure wall (see also section 9.) on a length of about $50 \mathrm{~m}$ which also seems to have covered Tomb XI. ${ }^{270}$

Whilst the pottery repertoire is clearly dominated by 'BB jars' (these are discussed below), there were several other ceramic types belonging to the general time horizon of the use of the temple (Fig. 36; 37; 38.a). A selection will be presented here. Also a number of red and black painted and blue painted potteries was found in and around this tomb but these sherds will be discussed separately (see Fig. 48).

\section{Various fabrics - So-called Beer Bottle after Holthoer (BB jars, Fig. 36.a-c)}

No. 8713 (L2), rd.=8.0-7.0, max.d.=15.3, bd.=7.0, ht. $=24.5$, Nile B2, section not visible, intact, surface uncoated (7.5YR5/4 brown), wheelmade (W1), base string cut (base type C), vessel warped, weight $1.0 \mathrm{~kg}$.

No. 8712 (L2), rd.= 8.5-7.0, max.d.= 15.5, ht. $=$ 26.0, Nile B3, section not visible intact, surface uncoated (7.5YR5/3 brown), wheelmade (W1), base string cut and edges of base cut subsequently (Base Type A), vessel cannot stand up, vessel warped, weight $1.5 \mathrm{~kg}$.

\section{Open vessel forms}

Many fragments of open vessels with red-splash decoration $^{271}$ were found. They may show direct rims, direct incurved rims, thickened rims and folded rims as well as a variety of base forms (round, flat and ring). Interestingly no material with black rims came to light there, which is quite rare in the temple material overall either due to chronological or other, perhaps social or organisatorial, reasons. ${ }^{272}$ The typical open vessels are made of Nile B2 or B3 with slightly incurved direct rims. ${ }^{273}$

Nile B3 - Red splash decorated dish (Fig. 36.d) R4/5-L9-17, rd.= 19.5, bd. $=7.2$, ht. $=5.8-6.8$, Nile B3, surface uncoated (2.5YR6/6 red) with red

\footnotetext{
271 Aston 2006.

272 Perhaps pottery with black rims had no specific use within the temple.

273 SeILER 2005, 140-142; Aston 2003, fig. 1.a-b.
} 

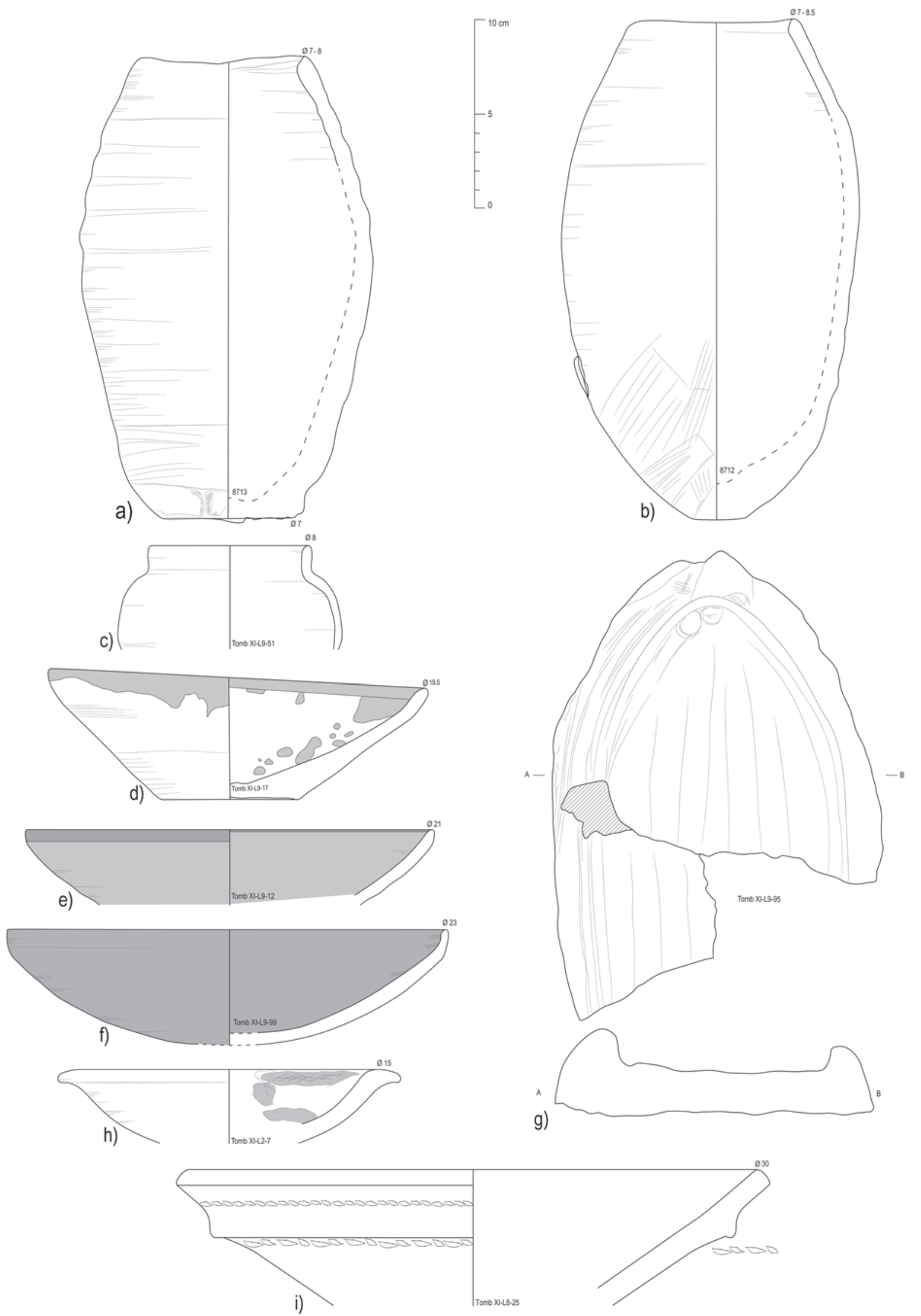

Fig. 36 Pottery from Tomb XI, New Kingdom, drawn by B. Bader, inked by E. Schuster. 
slipped rim and splash decoration (10R5/8 red), wheelmade, base cut with string.

\section{Nile B2 - Dish with incurved rim (Fig. 36.e)}

R4/5-L9-12, rd.= 21.0, pres.ht.=4.0, Nile B2, red slip on interior and exterior with darker red rim band, wheelmade.

\section{Nile B2 - Dish with upright rim (Fig. 36.f)}

R4/5-L9-99, rd. $=23.0$, pres.ht. $=6.1$, Nile B2, surface red slipped on interior and exterior (7.5YR4/6 red), polished (rilling lines are visible under polishing but not the polishing strokes), wheelmade, base wheelmade.

\section{Nile B2/C1 - Oval trays handmade (Fig. 36.g)}

The most remarkable observation to be made of this pottery type is that it has been handmade on the ground with a low ledge around an oval base. ${ }^{274}$ Not much time was spent on removing the traces of the manufacturing process so it seems that it was not important that these vessels looked appealing. In the tomb of Rekhmire, a vizier in office during Thutmosis' III reign, such vessels are shown in a food production scene ${ }^{275}$ in combination with the only other handmade vessel type occurring, namely the large white washed storage jars with obvious traces of manufacture (see below, Fig. 37.e).

No. R4/5-L9-95, rd.= oval, ht. $=4.5$, Nile B2/C1, surface uncoated (2,5 YR 5/6 red), handmade on ground.

\section{Nile B2 - Burner (Fig. 36.h)}

Burners are usually round based with a ledge rim and obvious resin and burning traces. Such pottery occurs already in the Ahmose complex at Abydos, ${ }^{276}$ although the contextual information is not unequivocal and the range seems to cover the entire New Kingdom. ${ }^{277}$

No. R4/5-L9-99, rd.= 15.0, pres.ht.= 3.9, Nile B3 with abundant quartz, surface uncoated, wheelmade, stained with burning of solid material and soot marks.

\footnotetext{
274 Another example in BADER 2015, fig. 6.28.

275 Cf. Nina de Garis Davis' painting in the Metropolitan Museum, reg. no 31.6.30.

276 BuDKa 2006, Fig. 3-4.

277 Pumpenmeier in: Dreyer et al. 1998, 133, fig. 27, top row. See also the foundation deposit in the mortuary temple of Sethos I, Myśliwiec 1987, 25-39, cat. nos 52-53.
}

\section{Nile B3 - Large ledged dish (Fig. 36.i)}

Such dishes occur quite frequently and exhibit string impressions above and below a thickened ledge.

R4/5-L8-25, rd.= 30.0, pres.ht.= 7.3, Nile B3, surface uncoated, wheelmade with two rows of string impressions.

\section{Closed vessel forms}

As mentioned before the corpus of closed vessel forms is dominated by 'BB jars', but there are some other vessel shapes represented in the fill of the tomb.

The pottery corpus of the $18^{\text {th }}$ Dynasty contains parallels for these vessel types: a neckless jar (Fig. 37.a) with upright direct rim, ${ }^{278}$ a jar with slightly tapering neck and without shoulder (Fig. 37.b) ) $^{279}$ and shouldered jars (Fig. 37.c $\mathrm{c}^{280}$; Fig. 37. $\mathrm{d}^{281}$ ). Beside the vessels mentioned below (Fig. 38) there are several fragments of large jars that were roughly scraped on the exterior from the maximum diameter towards the base, two type variants of which have already been published. ${ }^{282}$ Also a few rim fragments were found that belong to storage jars with folded rims, but so little of the body was preserved that they will not be shown here. The rim of the large storage jar (Fig. 37.e) finds close parallels in the New Kingdom. ${ }^{283}$

R4/5-L12-31, rd. $=8.0$, pres.ht. $=10.5$, Nile B3, surface uncoated, wheelmade.

$\mathrm{R} 4 / 5-\mathrm{L} 12-30, \mathrm{rd} .=11.0$, pres.ht. $=14.4$, Nile B2, hard fired, surface uncoated, wheelmade (strong rilling all over).

R4/5-L9-82, pres.ht. $=21.5$, Nile B2/C1, surface uncoated with drops of white slip irregular, wheelmade (rilling all over).

R4/5-L9-25, rd. $=5.0$, pres.ht. $=8.7$, Nile B2, fine variant, surface red slipped on exterior down to just below shoulder, wheelmade in at least two parts.

R4/5-L10-19, rd. $=27$, pres.ht. $=19.2$, Nile B3, hard fired, surface white slipped on exterior an rim on interior, wheelmade, at beginning of the shoulder hand manufacture clearly visible.

\footnotetext{
278 Holthoer 1977, 155, pl. 34, GJ 1, IR/0/f-g.

279 Holthoer 1977, JW, 162-163, pl. 38; Aston 2003, fig. 3.35, phase $2 \mathrm{~A}$ - Thutmosis III.

280 Aston 2003, fig. 3.

281 Probably Holthoer 1977, 164, pl. 39-40, NF 1 IR/0/c-d.

282 BADER 2015, fig. 5.24, 6.25.

283 E.g. Brack/Brack 1977, Taf. 63.1/43.
} 

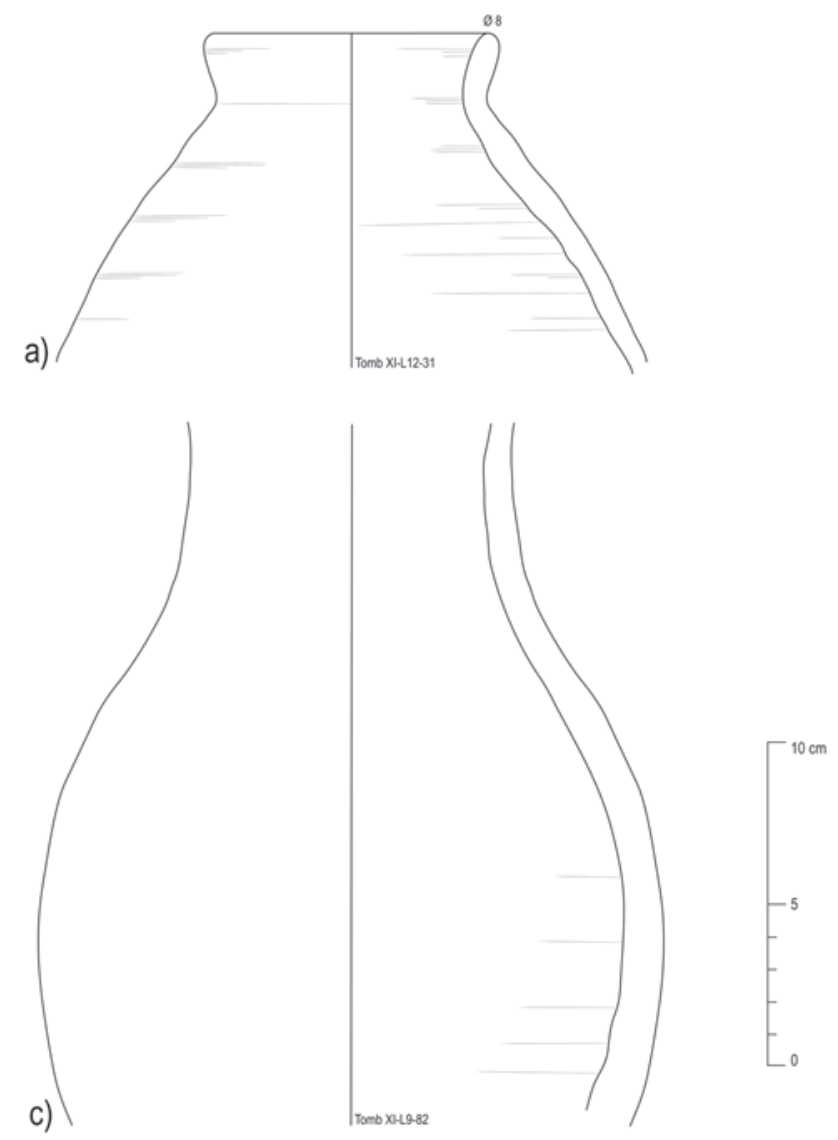

b)
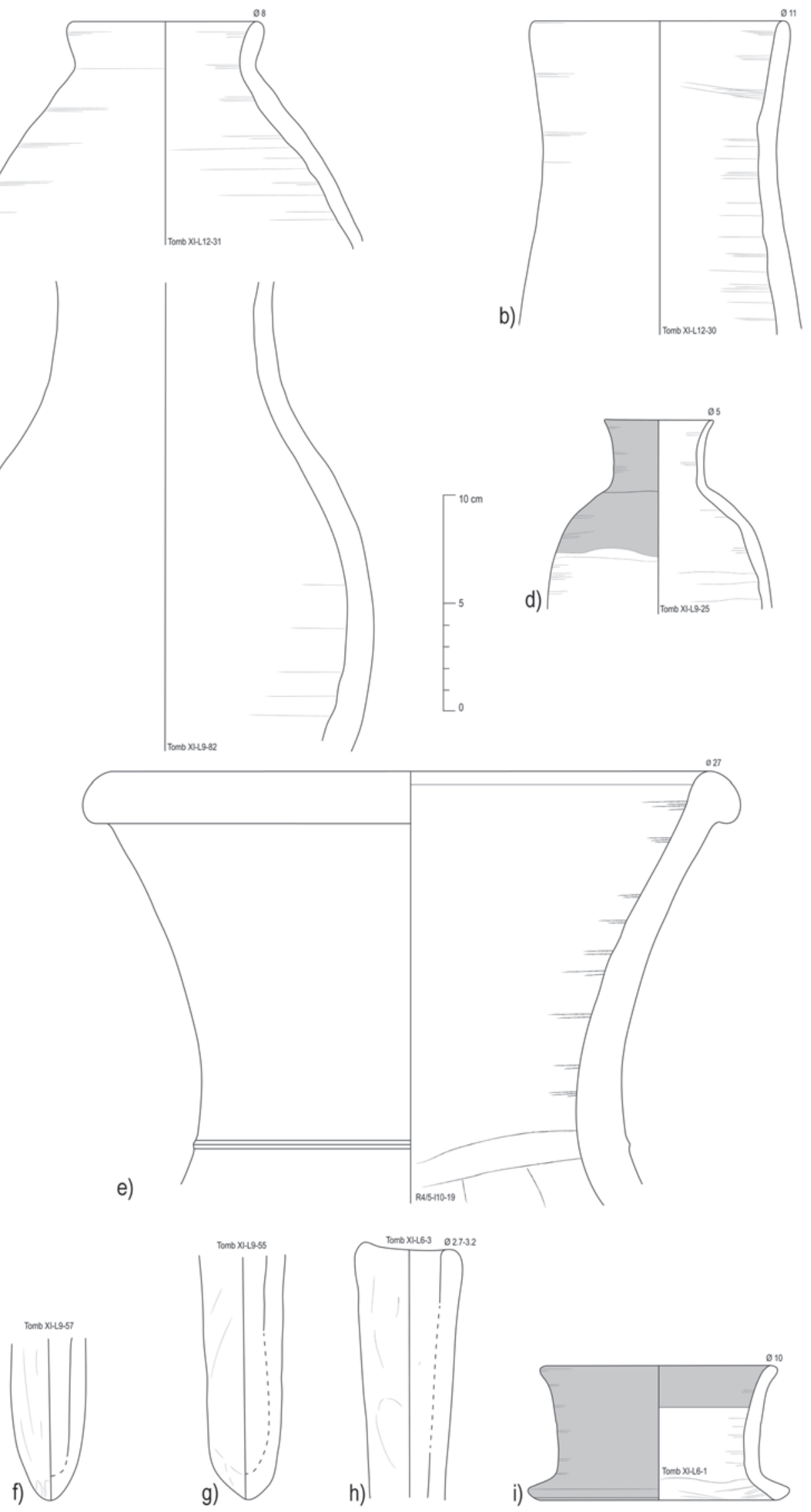

Fig. 37 Pottery from Tomb XI, New Kingdom, drawn by B. Bader, inked by E. Schuster. 
a)
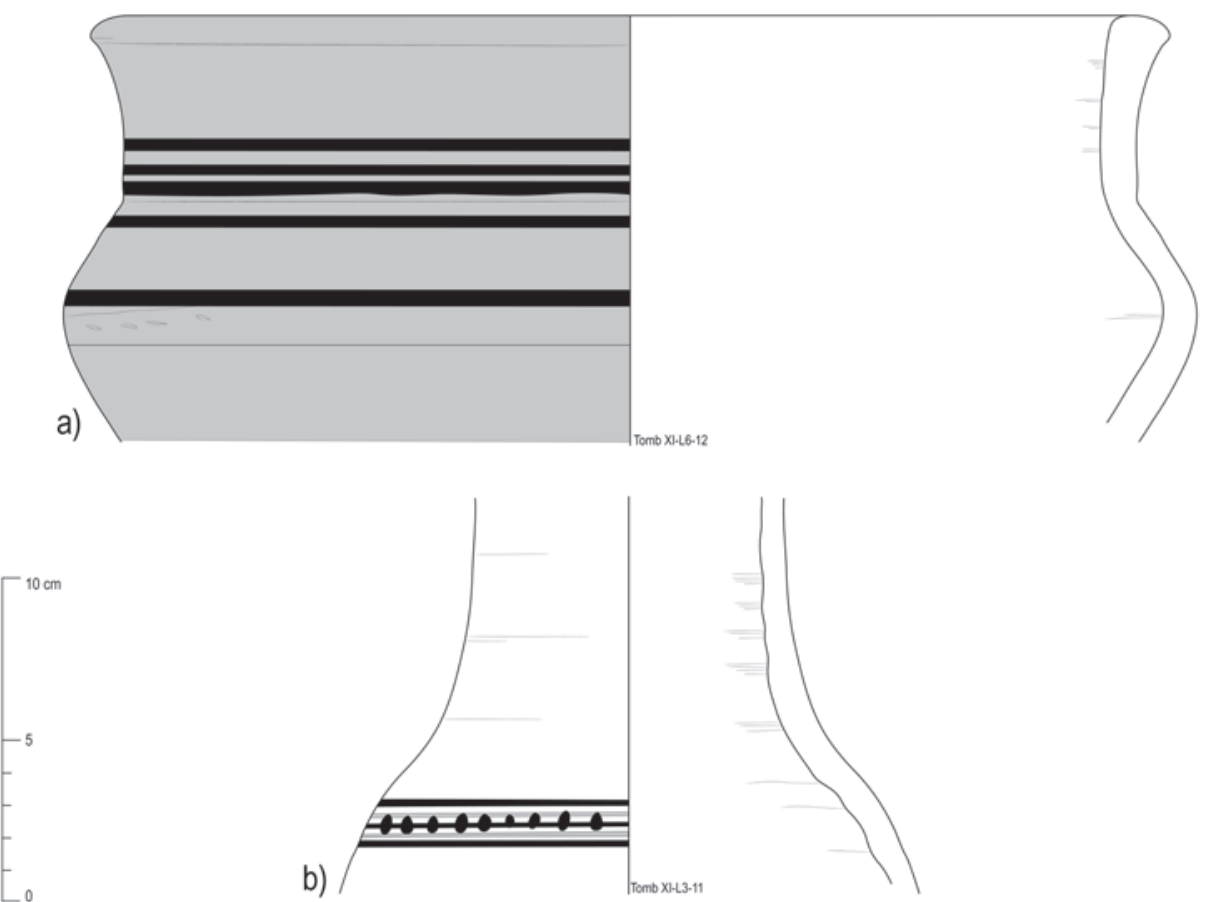

b)
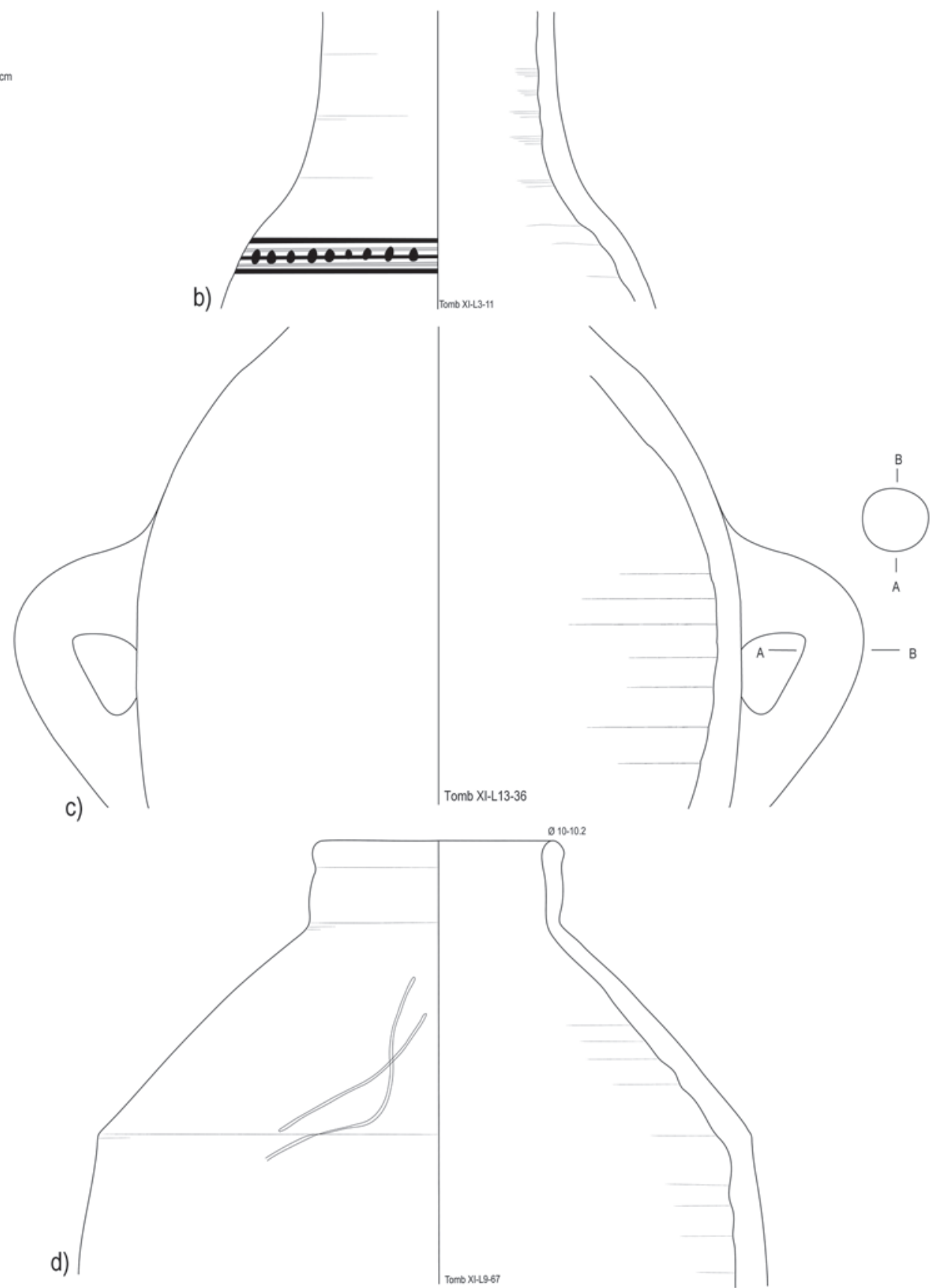

Fig. 38 Pottery from Tomb XI, New Kingdom, 25th Dynasty, drawn by B. Bader, inked by E. Schuster. 


\section{Bread moulds (Fig. 37. f-h)}

The bread moulds found in the tomb belong to a long thin and pointed variety, sometimes with one or two finger impressions at the bottom. In one example several thin layers of cream of clay ('Schlicker') with abundant mica was still preserved. The moulds were made over a core and the finger marks on the exterior are clearly visible. ${ }^{284}$

R4/5-L9-57, pres.ht. $=7.3$, Nile B3, surface uncoated, handmade over core, two finger impressions on the base.

R4/5-L9-53, pres.ht. $=7.3$, Nile B2, surface uncoated, handmade over core, smoke stained on exterior.

$\mathrm{R} 4 / 5-\mathrm{L} 6-3, \mathrm{rd} .=2.7-3.2$, pres.ht. $=11.4$, Nile B3, handmade over core, remains of fine cream of clay in several layers inside.

\section{Nile B2 - Ring stand (Fig. 38.i)}

One complete profile of a ring stand was recorded and it belongs to the higher variety of the New Kingdom with a direct slightly out-turned rim. ${ }^{285}$

R4/5L6-1, rd. $=10.0$, bd. $=11.0$, ht. $=6.2$, Nile B2, surface red slipped on the exterior and the rim on the interior, wheelmade, base scraped with a tool on the interior.

\section{Marl clay pottery (Fig. 38.a-b)}

Beside uncoated body fragments of Marl A4 vessels very few diagnostics were found, but some Marl clay fabric pottery was decorated. Among those was the typical Thutmosid red and black decoration of lines and dots also known as 'swallows on a wire' pattern ${ }^{286}$ or dot-lines ${ }^{287}$, and black lines $^{288}$ but body fragments made of Nile clay fabrics also showed such decoration.

R4/5-L6-12, rd.=30.0, pres.ht. $=13.1$, Marl A2, very dense, surface with scum and black painted horizontal lines, wheelmade, perhaps rim tick on the top of the rim.

R4/5-L3-11, pres.ht.= Marl A2, surface uncoated, with red and black painted decoration, wheelmade.

\footnotetext{
284 JACQUET-GORDON 1981, fig. 5.1-8.

285 Holthoer 1977, 76, pl. 15, TU 4 low.

286 Bourriau et al. 2005, 120; Rzeuska 2001, 301-303; Hope $1987 \mathrm{a}, 109$.

287 Holthoer 1977, 55-56; for the type: Holthoer 1977, pl. 39, NJ 3 IR/4D/g-h.

288 Holthoer 1977, 112, pl. 24, CC 3 IIR/3D/f-g.

289 Aston 2004a.
}

Imported pottery Amphora shoulder with handle (Fig. 38.c)

This amphora shoulder is very much rounded, with the section of the handle almost circular. The fabric of the piece is shown in Fig. 1.aa. From this fragment it is not possible to be certain whether the type belongs to the Middle or the Late Bronze Age. For the late Bronze Age the shoulder is very round and the handle sections tend to be oval, suggesting rather a Middle Bronze Age date. ${ }^{289}$

R4/5L13-36, pres.ht. $=19.6$, Foreign Fabric 7, surface obscured by hard white crusts.

\subsection{3.) Later Material (Fig. 38.d, 39.a-b)}

Only a few definitely post-New Kingdom vessels were present and they will be discussed in the following. Most of these have abundant parallels all over Egypt and can therefore be well dated. A Nile clay fabric jar most likely dateable to the $25^{\text {th }}$ Dynasty $^{290}$ (Fig. 38.d) was found in Layer 9, whilst a Marl clay storage jar type (Fig. 39.a) occurs frequently in the Late Period. Comparable examples were found in Thebes at Dra Abu el-Naga, ${ }^{291}$ the mortuary temple of Merenptah ${ }^{292}$ and in the priests' houses in Karnak. ${ }^{293}$ Beside a filter neck of a Marl clay qulla, the latest identifiable vessel is without doubt the tagine-like cooking vessel from Layer 8 . With this kind of material it is difficult to know whether it belongs to the $18^{\text {th }}-19^{\text {th }}$ century $\mathrm{AD}$ or an even later phase.

R4/5-L9-67, rd. $=10.0-10.2$, pres.ht. $=18.2$, Nile B2 with abundant quartz, hard fired, surface covered sloppily with a white slip on the exterior, wheelmade (W1-2), postfiring mark on the shoulder.

R4/5-L8-28, rd. $=9.0 \mathrm{~cm}, \max . \mathrm{d} .=22.0$, ht. $=$ 52.3, Marl A4, variant 2, extremely dense, section uniform reddish orange, surface covered by thick greenish scum, wheelmade.

$\mathrm{R} 4 / 5-\mathrm{L} 8-27, \mathrm{rd} .=31.0$, pres.ht. $=10.3$, fabric with lots of angular stones, ${ }^{294}$ section dark brown, surface burnished on interior and exterior, burnt, handmade with vertical incisions along the ledge on the exterior.

\footnotetext{
290 Polz et al. 2003, 364, fig. 19.1, late $8^{\text {th }}$ and $7^{\text {th }}$ century.

291 Polz et al. 2003, fig. 19.3, late $7^{\text {th }}$ and $6^{\text {th }}$ century BC.

292 Aston 2008, pl. 144, 276-278, 27 $7^{\text {th }}$ Dynasty.

293 MAsson 2011, pl. 2, 25 ${ }^{\text {th }}$ to $26^{\text {th }}$ Dynasty.

294 The fabric looks very similar to one found at the Luxor Town Mound project of AERA in collaboration with ARCE, in which Bettina Bader took part in 2010.
} 

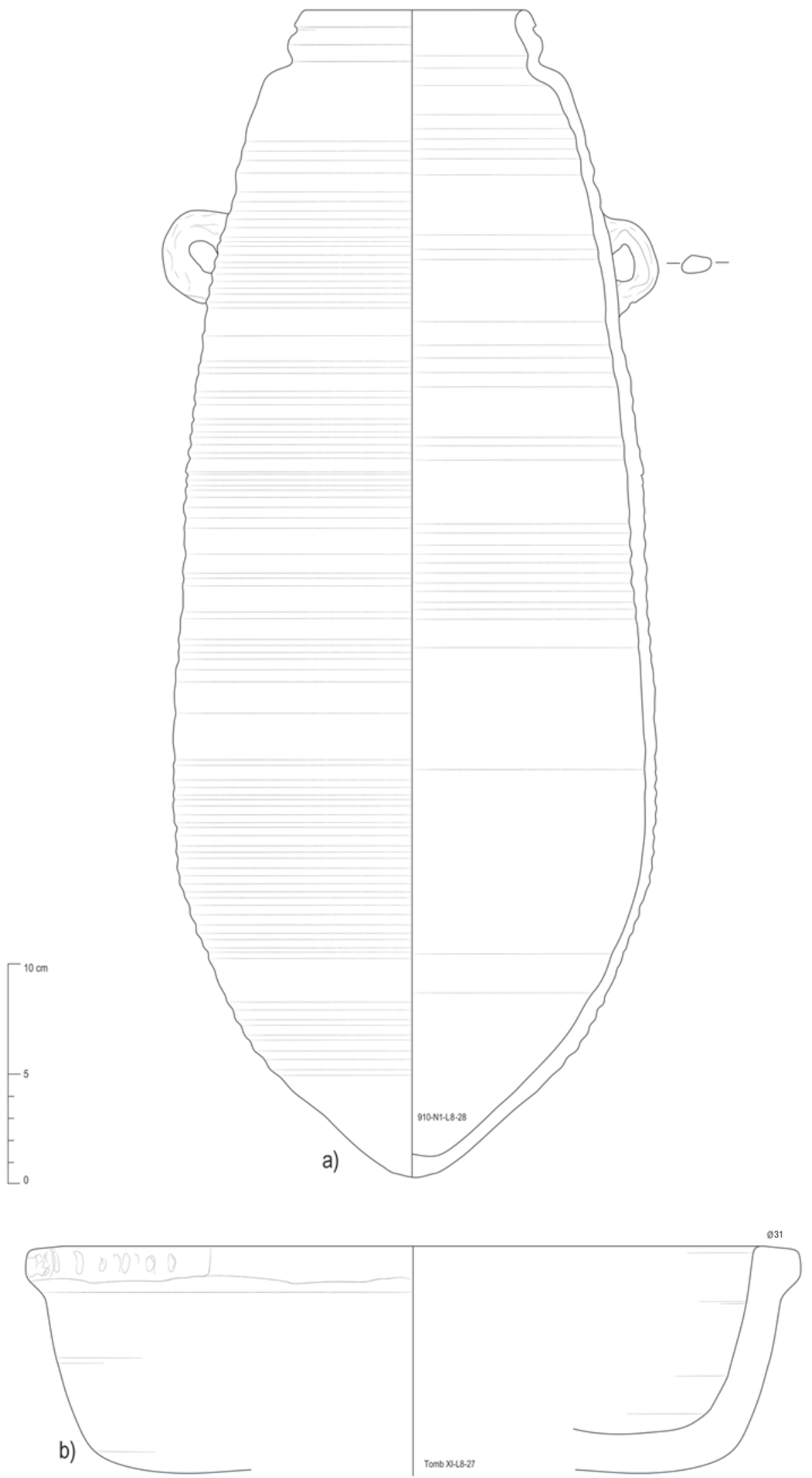

Fig. 39 Pottery from Tomb XI, later material, drawn by B. Bader, inked by E. Schuster. 

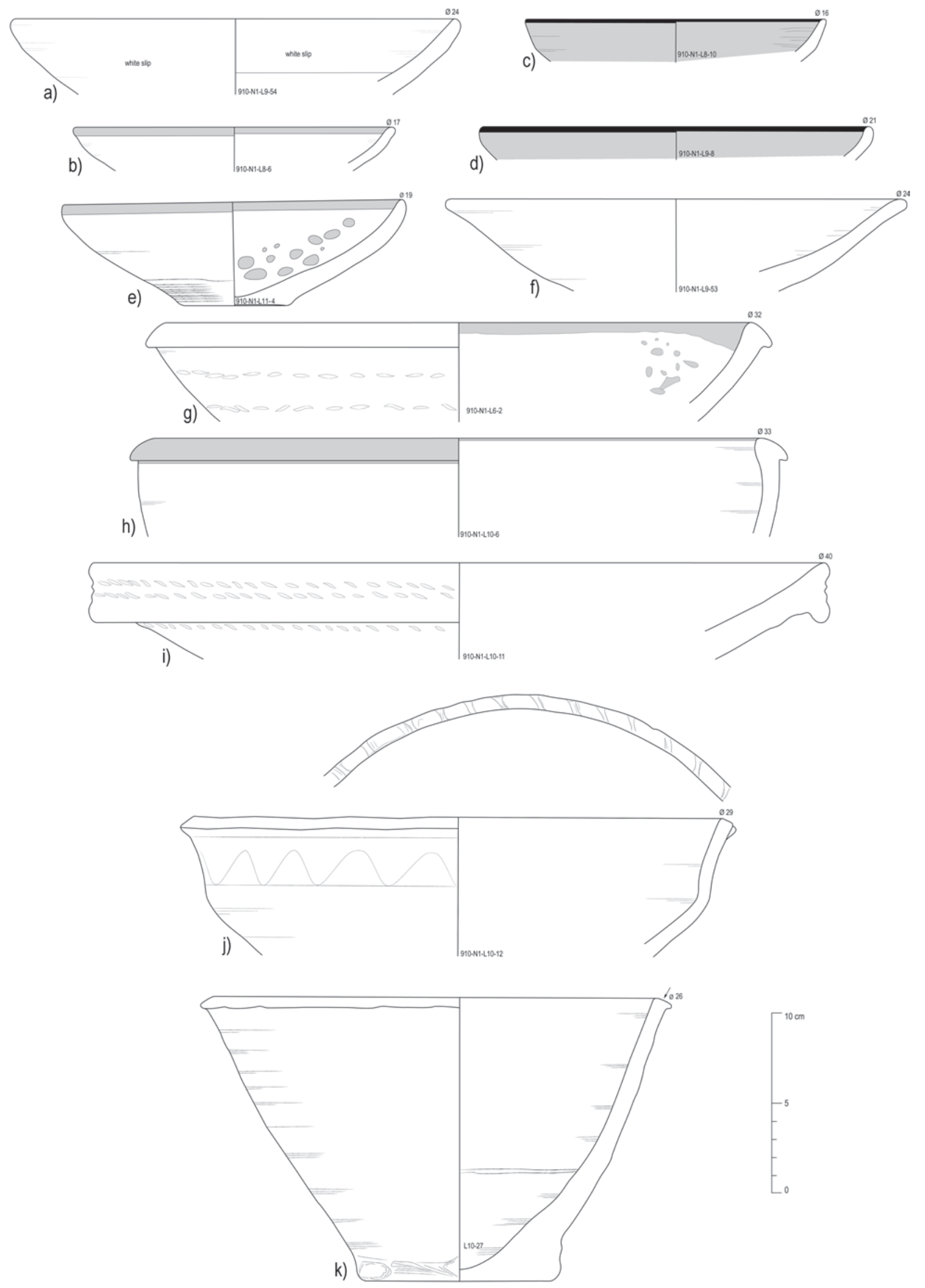

Fig. 40 Pottery from foundation deposit 910-N1, reign Thutmosis III, drawn by B. Bader, inked by E. Schuster. 


\section{7.) The pottery corpus contemporary with the use life of the temple of Thutmosis III}

\section{1.) The foundation deposit (square 910-North1)}

This deposit was situated in the second courtyard. It was placed to the north of the doorway that led into this courtyard. As it was next to the interior side of the western enclosure wall, ${ }^{295}$ it was probably signalling the position of this wall.

The fact that the ceramic material was very broken and could only be reconstructed to complete profiles in some cases, indicates that the deposit was not intact. In spite of this the material gives a homogeneous impression in terms of chronology. The only fragment that 'sticks out' is a thick-walled blackened cooker of Nile clay fabric burnished on the interior and deeply combed on the exterior. ${ }^{296}$ Most interestingly a dish fragment with the painted cartouche possibly of Thutmosis III belongs to the assemblage. ${ }^{297}$ The deposit was excavated in several layers L4, L6, L8, L9, L10 and L11 and analytic work on the pottery revealed several joins across these contexts: L4/L6, L6/L8, L8/L9/L10, L8/L9, L8/L10, L9/L10. Interestingly there were no joins between L11 and the remaining layers but the same vessel types occur. Because these cross joins proved the connection of the layers the pottery will be presented in corpus form. ${ }^{298}$

Pottery types occurring in fragments but not included in the figures comprise the hand formed sooted leg/ear of a fire $\operatorname{dog},{ }^{299}$ the base of a narrow bread mould (cf. Fig. 37.f-h), one fragment of a ring stand with a white slip, two very small jar rims and bases of model vessels and one very thick walled base filled with white plaster from L10. ${ }^{300}$

All in all the pottery of the deposit is very similar to that which Ricke had discovered during his work in the temple of Thutmosis III, ${ }^{301}$ although the range of types he shows is much more restricted. The disturbance of the deposit 910-N1 may ultimately be the reason for this. Another, dis-

\footnotetext{
295 Seco Álvarez 2015, 117-118, fig. 14; Seco Álvarez/MartiNEZ BABOn 2015a. Due to error Bettina Bader was omitted as co-author of this last paper. A correction slip will be published in the next volume of the journal Memnonia.

296 It is possible that this fragment represents a Kerma cooking pot. D. Aston, personal communication.

297 BADER 2015, 298, cat.no.1, fig. 1.1

298 In BADER 2015 some of the vessel types are published.

299 Aston 1989.
}

turbed foundation deposit for a pillar in the temple was found by Borchardt in 1934. In addition to some joints of a calf (head and leg), only pottery was found, which overall is very similar to 910 N1. The completely preserved vessels were published in photographs (16 out of about 40 in total) which included slender bottles, 'BB jars', incurved bowls with direct rims, straight walled bowls with direct rims and narrow New Kingdom bread moulds. ${ }^{302}$

A number of foundation deposits of the Thutmosid period are known from other sites but are usually only partially published. ${ }^{303}$ Comparing the pottery from 910-N1 with other contemporary deposits it becomes obvious that in 910-N1 the model pottery is in the minority. The largest proportion of vessels is taken up by flower pots $\left(\mathrm{MNI}^{304} 5\right)$ and 'BB jars' (MNI 31), which may be a further hint at disturbance. However, the ordinary pottery from foundation deposits is rarely published in full so that comparative interpretations are difficult. There are certainly differences in the composition of the types of objects as well as in the pottery types, which need to be addressed by further research.

\section{Open shapes \\ Nile B2 - Dishes/bowls with direct incurved rims (Fig. 40.a-e)}

This vessel type represents one of the most ubiquitous pottery types occurring in the temple material. There are variations represented in the foundation deposit comprising sherds with red slipped surface and red rim, uncoated and red rim, uncoated and white rim (wide), red slipped with black rim (in two instances) and red slipped rim with red splash decoration. Ring pattern burnishing also appears. The occurrence of these subtypes is spread over all the layers. The usual fabric used for these dishes is Nile B2 although Nile B3 and once Nile B4 and even Nile $\mathrm{C}$ were also observed. Bases probably belonging to this type are usually raised bases produced on the wheel (after turning

\footnotetext{
300 Similar to BAder 2015, fig. 5.24 and 6.25

301 Ricke 1939, Taf. 12, although he only published one drawing of each type.

302 FAKHry 1937, 28-30, pl. II-b-III.

303 For a list of foundation deposits dating to the reigns of Hatsheptsut and Thutmosis III up to 1973 Weinstein 1973, 92-141; 149-196. Since then in Thebes SzAFranski 1997; Mensan 2007.

304 Minimum Number of Individuals.
} 
them over). But only the bases of red splash bowls could be identified unequivocally and they were all made on the wheel. The diameters of the rims range from 16.0 to $24.0 \mathrm{~cm}$, whilst the base diameters are usually around 6.0 or $7.5 \mathrm{~cm}$. Red splash bowls were the most common variant. ${ }^{305}$

910-N1-L9-54, rd.= 24.0, pres.ht.=4.1, Nile B2, surface uncoated with wide white rim on interior and white slip on exterior, wheelmade.

910-N1-L8-6, rd.= 17.0, pres.ht. $=2.4$, Nile B2, very fine variant, surface red slipped on exterior and interior, red rim, wheelmade.

910-N1-L8-10, rd.=16.0, pres.ht.= 2.3, Nile B2, very fine variant, surface red slipped on exterior and interior, black rim, on interior ring pattern burnishing, wheelmade.

910-N1-L9-8, rd. $=21.0$, pres.ht. $=1.8$, Nile B2, surface red slipped on exterior and interior, black rim, wheelmade.

910-N1-L11-4, rd. $=19$, bd. $=6.0$, ht. $=5.8$, Nile B2, section Fig. 1.k, surface uncoated with red slipped rim and red splash decoration on exterior, wheelmade, base wheelmade.

\section{Nile B2 - Dishes with direct out-turned rim (Fig. 40.f)}

This shape variant is much rarer and only represented as rim sherds (L6-8, L8-8-9 and L9-53). The fabric is mostly Nile B2 although Nile B3 also appeared once. The dishes were uncoated except one with a red rim.

910-N1-L9-53, rd.= 24.0, pres.ht. $=4.9$, Nile B2, surface uncoated, wheelmade.

\section{Nile B2 - Bowls with folded rim (Fig. 40.g-h)}

Only four rim fragments can be assigned to this type, whilst the depth of these vessels obviously differs. Their rim diameters are all more than $26.0 \mathrm{~cm}$ and even over $30.0 \mathrm{~cm}$. The surface treatment was mostly uncoated except for the redsplash bowl. The fabrics used belong to the Nile B2 group.

910-N1-L6-2, rd. $=32.0$, pres.ht. $=5.5$, Nile B4, surface uncoated with red-splash decoration, wheelmade.

910-N1-L10-6, rd.= 33.0?, pres.ht. $=5.5$, Nile B2, surface uncoated only lip red slipped, wheelmade.

\footnotetext{
305 Aston 2006.
}

Nile B4 - Large Bowl with folded rim (Fig. 40.i)

One large bowl with folded rim and several rows of rope impressions belongs to the assemblage.

910-N1-L10-11, rd.= 40.0, pres.ht. $=5.3$, Nile B4, surface uncoated, wheelmade.

\section{Nile B2 - Carinated bowl with pie-crust rim (Fig. 40.j)}

This vessel type is reminiscent of Second Intermediate Period types and may be a further development of it.

910-N1-L10-12, rd.=29.0, pres.ht.= 7.7, Nile B2, surface eroded, wheelmade, rim pinched with fingers.

\section{Nile B2 -Flower pots (Fig. 40.k)}

Among the open shapes this vessel type occurred most frequently in the foundation deposit. It is the hall mark of mid- $18^{\text {th }}$ Dynasty pottery. ${ }^{306}$ Some bases found had a pre-firing hole in the middle of the base, but non-pierced examples also exist. All bases were string cut. None of the vessels had holes in the sides of the vessel. The rims were mostly trimmed with a tool and the trimming created a small 'lip' either on the inside or the outside of the rim. More rarely the rim was just square in section or rounded. One example had a folded rim, whilst another showed a flaring one. Slightly triangular rims also occurred. The fabric variants are Nile B2 and Nile B3, and the surface of the vessels is usually uncoated. The bases were all string cut, the quality of the manufacture varying widely. Again all layers contained fragments of such vessels. The Metropolitan Museum of Art, New York shows one flower pot from a foundation deposit from Hatshepsut's temple at Deir el-Bahari corroborating the occurrence of this type in foundation deposits (27.3.421).

910-N1-L10-27, rd. $=26.0$, bd. $=10.0-11.0$, ht. $=$ 15.7, Nile B3, surface uncoated, smoothed inside, made in two parts, wheelmade, base string cut.

\section{Nile B2 -Small and medium conical cups (Fig. 41.a-c)}

This vessel type is reminiscent of a flower pot in terms of the manufacturing technology as well as in the general proportions. One complete profile from L11 and several rim fragments that may belong to the same type (L11, L9) were found in

\footnotetext{
306 Holthoer 1977, 83-86, FP, pl. 18; SäVe-SöDERBErgh/TroY 1991, 44, Type FP mid $18^{\text {th }}$ Dynasty.
} 

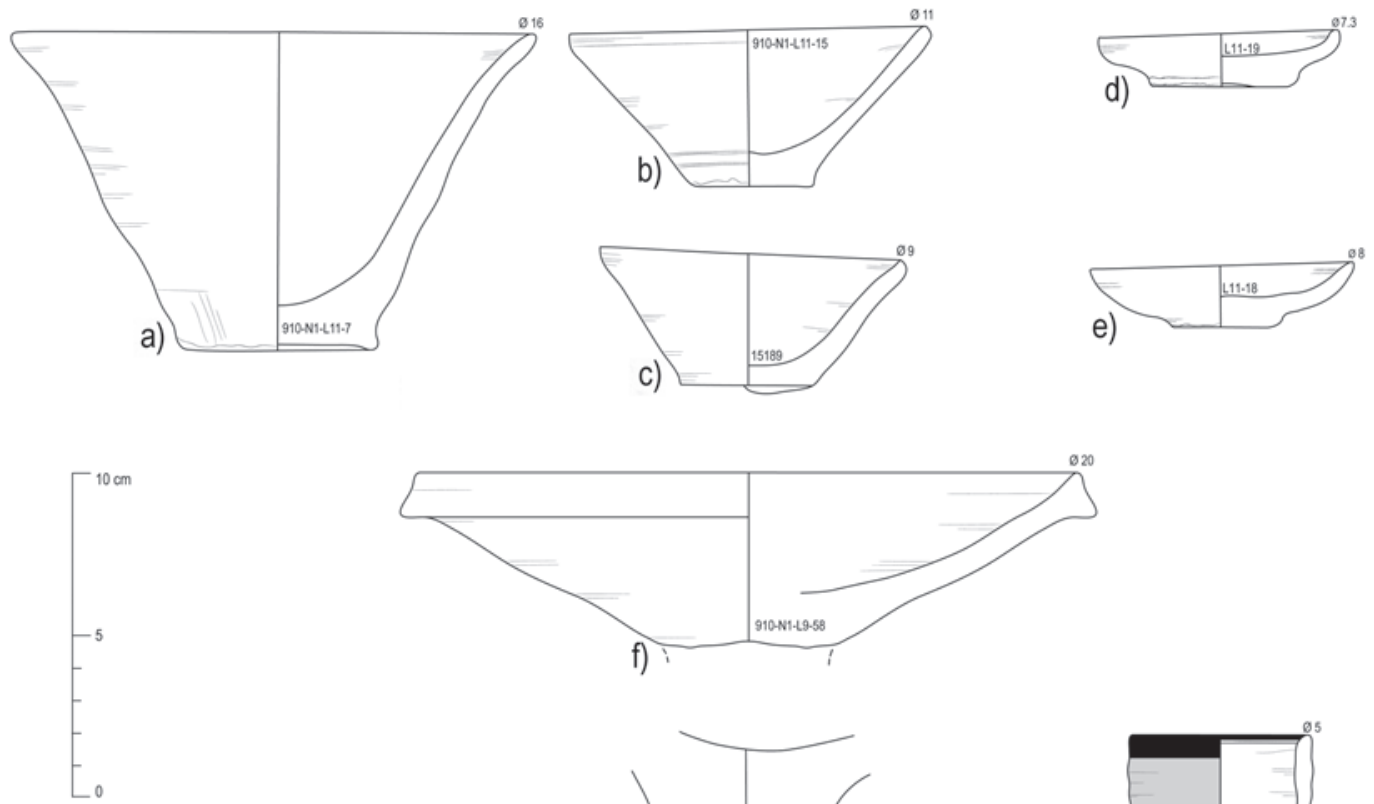

h)
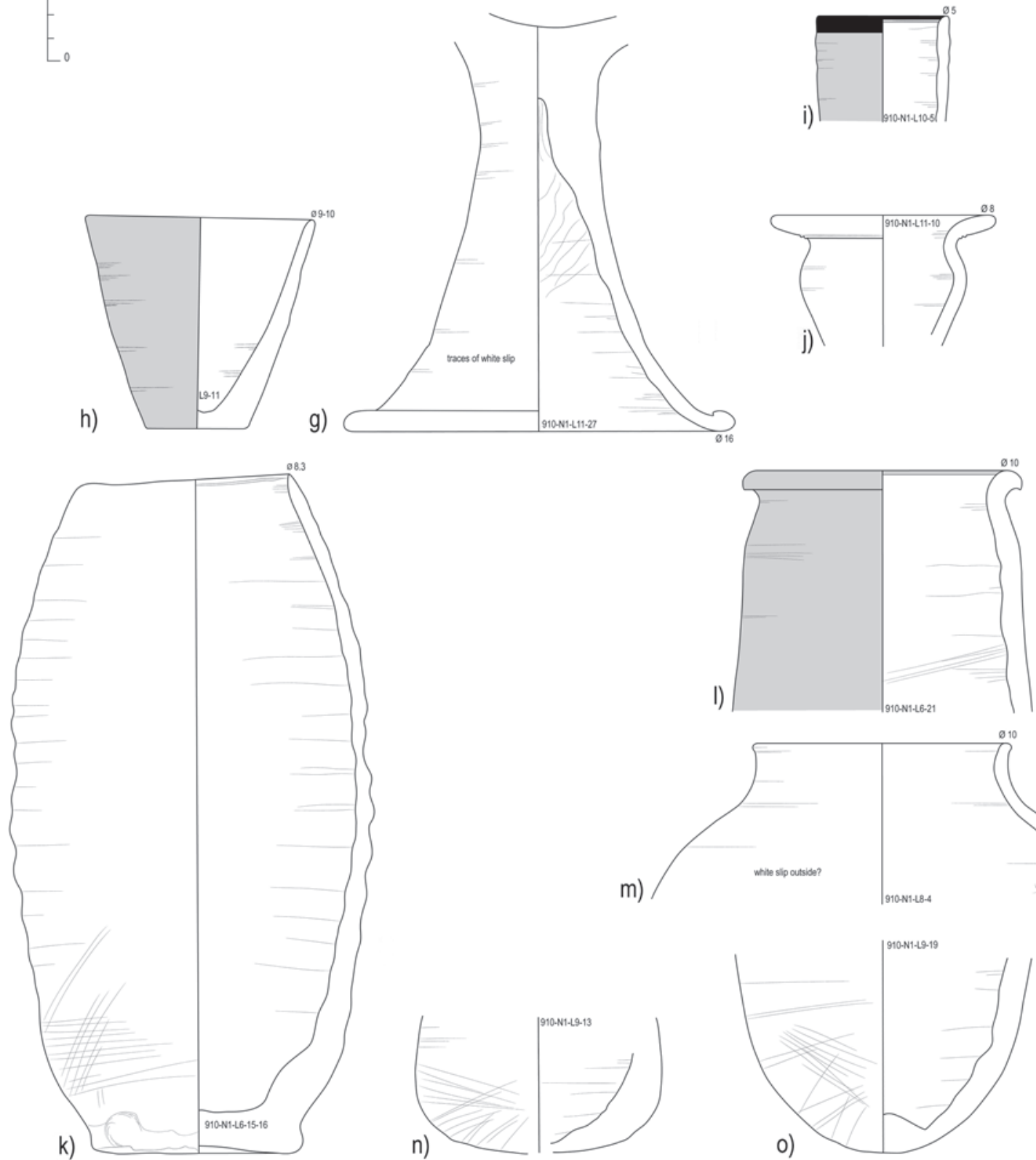

Fig. 41 Pottery from foundation deposit 910-N1, reign Thutmosis III, drawn by B. Bader, inked by E. Schuster. 
the deposit. An even smaller version was also found in two complete profiles. Note that the rim was also trimmed and the base string cut. Similar shapes are known from the foundation deposit at the Djeser-akhet platform in the temple of Hatshepsut at Deir el-Bahari but they are not exactly the same. ${ }^{307}$

910-N1-L11-7, rd. $=16.0$, bd. $=6.0$, ht. $=9.8$, Nile B2, surface uncoated, wheelmade, base string cut.

910-N1-L11-15, $\mathrm{rd} .=11.0, \mathrm{bd} .=4.0, \mathrm{ht} .=4.8$, Nile B2, surface uncoated, wheelmade, base string cut, some black material is stuck to the interior of the vessel.

No. 15189 (L11), rd.=9.0-9.5, bd.=4.0, ht.= 3.9-4.6, Nile B2/C1, section not visible, surface uncoated (5YR5/6 yellowish red), wheelmade, base string cut.

\section{Nile B2 - Small dishes (Fig. 41.d-e)}

These dishes are very reminiscent of Seiler's trend setters for the $18^{\text {th }}$ Dynasty. ${ }^{308}$

910-N1-L11-18, rd. $=7.0$, bd. $=4.5$, ht. $=2.0$, Nile

B2, surface uncoated, wheelmade, base string cut.

910-N1-L11-19 rd. $=8.0$, bd. $=3.0-3.5$, ht. $=1.7$, Nile B2, surface uncoated, wheelmade, base string cut.

\section{Nile B2 - Offering stands (Fig. 41.f, g)}

At least four offering stands were part of the assemblage because four mid pieces, where the feet and the dish meet, were found. The rims of these stands seem to have been folded and subsequently trimmed in the middle so that a depression resulted. One of the dish fragments showed traces of burning on the interior as well as some white substance adhering to it. Fragments of such offering stands were found in L9 and L11. ${ }^{309}$

910-N1-L9-58, rd. $=20.0$, pres.ht. $=5.4$, Nile B2, surface uncoated, smoothed on interior, rim trimmed with a tool, wheelmade.

910-N1-L11-27, bd. $=16.0$, pres.ht. $=17.1$, Nile $\mathrm{B} 2 / \mathrm{C} 1$, surface eroded, but traces of white slip on exterior, wheelmade, base wheelmade, middle of stand squeezed into shape.

\footnotetext{
307 SzAFrAnSKi 1997, fig. 2.1, 5, 8.

308 SeIler 2005, 140-141, Abb. 62.5-8.

309 Cf. Mensan 2007, 21-25, 23, photo at bottom of page, stands of similar shape.

310 SzAfranski 1997, fig. 2.7 with a red rim band.
}

Closed shapes

Nile B2 - Small beaker with flaring rim (Fig. 41.h)

This shape is generally replicated by one vessel from a foundation deposit at Deir el-Bahari, although the ware is different. ${ }^{310}$

910-N1-L9-11 rd. $=9.0-10.0, \mathrm{bd} .=4.5, \mathrm{ht} .=9.0$, Nile B2, surface red slipped on exterior, wheelmade, base string cut and well smoothed.

\section{Nile B2 - Small beaker jar with black rim (Fig.}

41. i)

Beakers in general occur quite frequently in the temple in several different wares: uncoated, red slipped on exterior, red slipped and burnished on exterior, and red slipped with a black rim band. But the latter are extremely rare within the temple, as far as it is known currently. ${ }^{311}$

910-N1-L10-5, rd. $=5.0 ?$, pres.ht. $=4.5$, Nile B2, surface red slipped on exterior and rim on interior, on top of this a black rim, wheelmade.

\section{Nile B2 - Small beaker/jar with carinated con- tour (Fig. 41. j)}

The rim fragment of this unusual vessel has a flaring rim with an S-shaped contour. Because it is not completely preserved parallels are hard to identify. Similar vessels may be represented in the foundation deposit of the tomb of Hatshepsut in the Valley of the Kings. ${ }^{312}$

910-N1-L11-10 rd. $=9.0-10.0$, pres.ht. $=5.5$, Nile B2, surface uncoated, wheelmade.

\section{Nile B2 - BB jars (Fig. 41.k)}

All the 'BB jars' found in the foundation deposit belong to the variety with direct incurved rim (see Fig. 7, 36.a-b). ${ }^{313}$ This type was the most numerous closed vessel type (MNI 31). The variants of Nile B clay include Nile B2, B3 and B4. The base varieties comprise Type $\mathrm{B}$ and $\mathrm{C}$ and very few of $\mathrm{D}$ (see below). The jars were mass produced and not of particularly good quality. Some of the bodies sported discoloured spots from touching the next vessel in the kiln, also hinting at mass production. The direct rims where either trimmed and very pointed at the end, slightly thickened or thinner than the vessel wall of the body.

\footnotetext{
311 SEILER 2005, Falttaf. 6.6-7, assigns them to the early $18^{\text {th }}$ Dynasty.

312 Davis/CARTer 1906, pl. XIV.9-10.

313 RicKe 1939, Taf. 12 last row, left.
} 
910-N1-L6-15-16, $\quad \mathrm{rd} .=10.0-12.0 \quad$ (warped), max.d. $=15.2$, bd. $=8.8$, ht. $=28.2-28.6$, Nile B2, surface uncoated, wheelmade, base string cut, vessel asymmetrical.

\section{Nile B2 - Cylindrical vessel (Fig. 41.l)}

This rim fragment may represent a variation of a 'BB jar', although no direct parallels are known. In the dumps north of the temple such a vessel with string-cut base was found. This means that bases of BB-jars and this type are undistinguishable.

910-N1-L6-14, rd. $=10.0$, pres.ht. $=10.2$, Nile B3, surface red slipped exterior and rim on interior, wheelmade.

\section{Nile B2 - Globular jar with modelled rim (Fig. 41.m)}

The preservation of the rim fragment suggests a globular jar. The top part includes one join line suggesting the piece belongs to a larger vessel. No immediate parallels from Thebes are known to us.

910-N1-L8-4, rd.= 10.0, pres.ht. $=7.0$, Nile C, surface white slipped?, made in at least two parts, wheelmade.

\section{Bases of closed vessels (Fig. 41.n-o, 42.c)}

A variety of different bases are shown to round off the picture of the vessel types found in the foundation deposit. Simple round bases occur as well as one bag-shaped type. Also interesting is the ovoid to ellipsoid vessel with a small flat base.

910-N1-L9-13, pres.ht. $=5.6$, Nile B2, surface uncoated, wheelmade, base scraped on exterior.

910-N1-L9-19, pres.ht. $=8.6$, Nile B3, surface uncoated, wheelmade, base scraped on exterior.

910-N1-L9-22, bd.=4.0, pres.ht.=17.8, Nile B2, surface light red slipped on exterior, wheelmade, made at least in two parts, base wheelmade with extra pad added as base.

Nile B2 - Medium slender bottles (Fig. 42.d-f) Such vessels were already found by Ricke in a foundation deposit. ${ }^{314}$ There he shows two different vessel types, which may well be represented in

\footnotetext{
314 Ricke 1939, Taf. 12, top row.

315 DAVIS/CARTER 1906, pl. XIV.11 but incomplete.

316 Mensan 2007, 22, top photo.

317 SzAFranski 1997, fig. 3.12.

318 DAVIS/CARTER 1906, pl. XIV.5 or 6.
}

this material. Altogether eight vessels and bases/ bodies of similar shape have been found (1 in L9, 7 in L11), which is very close to Ricke's seven examples of the two types. Similar vessel fragments are known from a foundation deposit of Hatshepsut in the Valley of the Kings, ${ }^{315}$ and at Karnak. ${ }^{316}$

910-N1-L11-31, rd.= 3.2, max.d. $=9.9$, bd. $=5.5$, ht. $=23.0$, Nile B2, section Fig. 1.j, surface uncoated, visibly wet smoothed, wheelmade, base string cut and trimmed.

910-N1-L11-33, max.d. $=9.7$, bd. $=5.0$, pres.ht. $=$ 21.8, Nile B2, surface uncoated, wheelmade, base string cut and trimmed.

910-N1-L11-35, max.d. $=8.2, \mathrm{bd} .=5.5-6.3$, pres. ht. $=14.0$, Nile B2, surface uncoated, visibly wet smoothed, wheelmade, base string cut, left rough.

\section{Nile B2 - Model vessel with incurved rim? (Fig. 42.g-h)}

To classify these rim fragments is quite difficult, but similar pottery exists in a foundation deposit at Deir el-Bahari. ${ }^{317}$ Possible parallels are from the foundation deposit of Hatshepsut's tomb in the Valley of the Kings. ${ }^{318}$

910-N1-L9-9, rd. $=5.0$, pres.ht.= 3.0, Nile B2, surface red slipped on exterior, wheelmade.

910-N1-L11-14, rd.= 5.0, pres.ht. $=3.8$, Nile B2, surface uncoated, encrusted with salt crystals, wheelmade.

\section{Marl A2 - Squat jar with black and red hori- zontal lines (Fig. 42.i)}

Red and black line decoration is extremely common in the reign of Thutmosis III, regardless if on Nile clay or Marl clay vessels. ${ }^{319}$

910-N1-L10-13, pres.ht.= 6.4, Marl A2 (greenish grey), surface very eroded, wheelmade.

\section{Marl A4 - Squat jar with handle(s) (Fig. 42.j)}

Such squat jars are quite rare in the Theban necropolis but are known to belong to the New Kingdom pottery corpus. ${ }^{320}$

910-N1-L6-20, pres.ht. $=9.0$, Marl A4, Section Fig. 1.p, surface uncoated, wheelmade.

\footnotetext{
319 Aston 2003, fig. 3.26, 28; Holthoer 1977, pl. 23, FO 2, pl. 31-32, CV 1 IIIP/3D/a-b; SÄVe-SöDerbergh/Troy 1991, 44, type FO2 dates to the mid $18^{\text {th }}$ Dynasty; 39 type CV1 decorated to the $18^{\text {th }}$ Dynasty.

320 Holthoer 1977, pl. 23, AH 1; SÄVe-SöDerbergh/Troy 1991, 28 , AH1 occurs rarely at Fadrus in the early to mid- $18^{\text {th }}$ Dynasty.
} 
Marl A4 - Large slender jar with grooves on neck (Fig. 42.a-b)

This type was already discussed (Fig. 13.g).

910-N1-L8-20-21, pres.ht. $=21.3$, Marl A4, surface uncoated, wheelmade, three grooves incised pre-firing on neck, base wheelmade with horizontal rilling, well smoothed.
Imported transport amphorae (Fig. 43.a-b)

Perhaps the least likely to belong to the foundation deposit are the fragments of imported amphorae, which were found in small quantities in all layers. The other material consisted only of non-diagnostic material: L6 -three sherds of FF2; L10 -two sherds of FF1 and two of FF2.
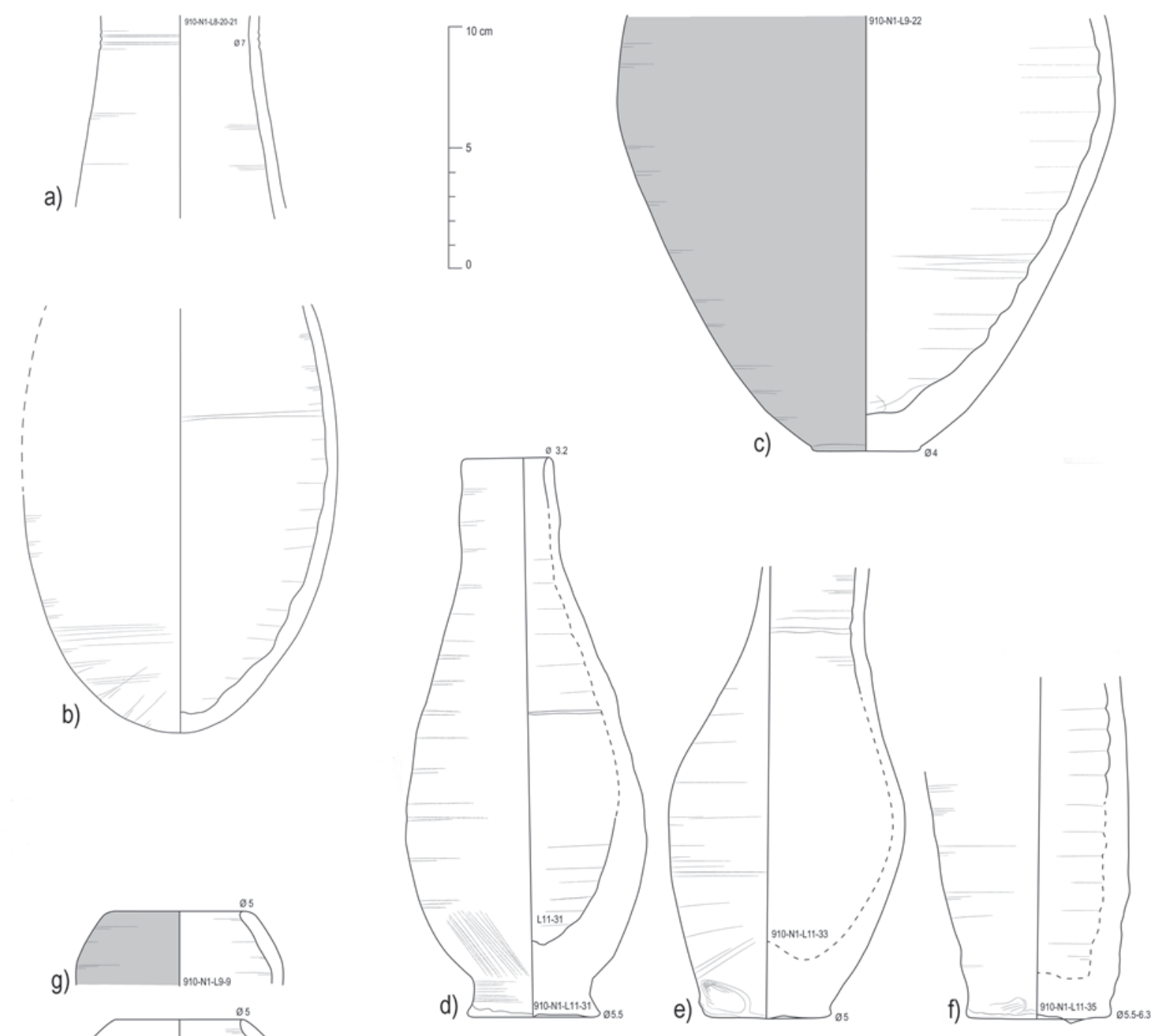

g)
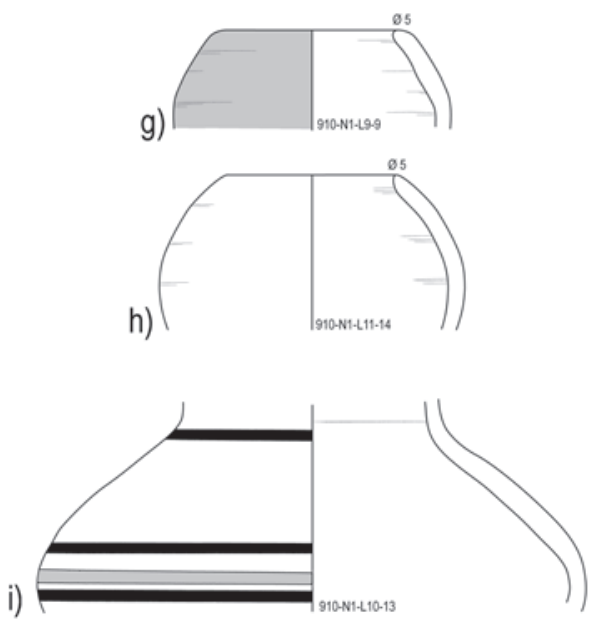
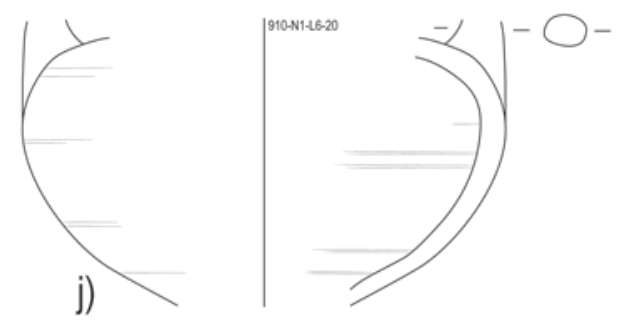

Fig. 42 Pottery from foundation deposit 910-N1, reign Thutmosis III, drawn by B. Bader, inked by E. Schuster. 
a)

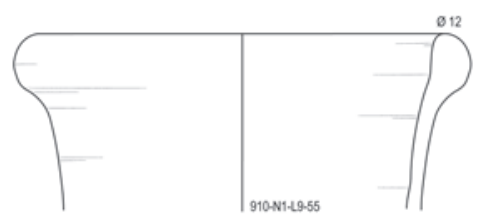

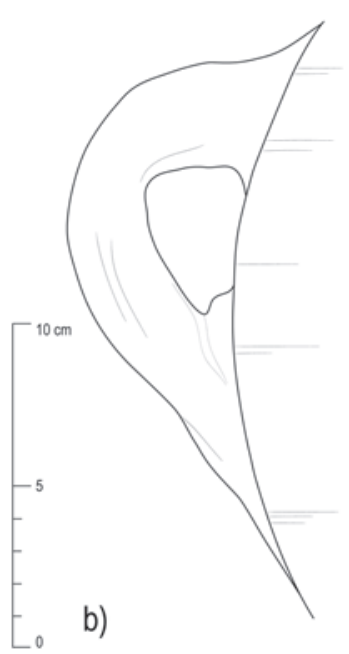

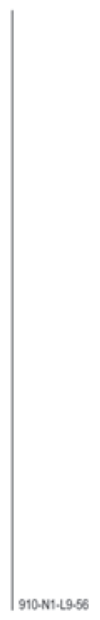

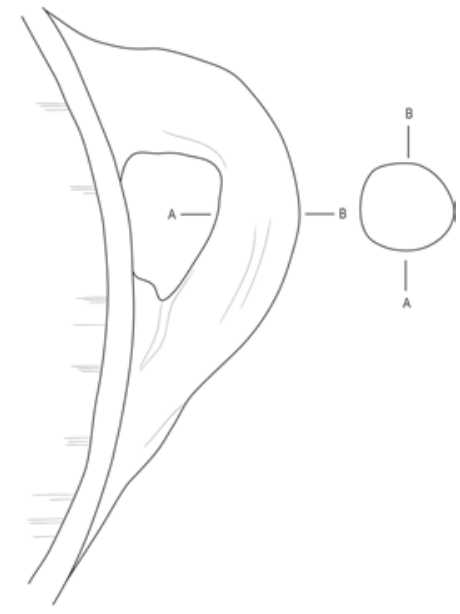

Fig. 43 Pottery from foundation deposit 910-N1, reign Thutmosis III, drawn by B. Bader, inked by E. Schuster.

910-N1-L9-56, pres.ht. $=$ 18.2, FF 1, surface uncoated (no scum), wheelmade, Fig. 1.t.

910-N1-L9-55, rd.= 12.0, pres.ht.= 5.5, FF 1, surface uncoated (no scum), wheelmade.

\section{2.) The 'BB jars'}

In the New Kingdom occupation of the site, the variety of Nile clay fabrics especially those used for the mass production of so-called 'Beer Bottles ${ }^{321}$ is extremely rich in variants and differs greatly in quality. The number and density of inclusions in such vessels varies from almost no visible inclusions, which is admittedly very rare, to sections that are full of inclusions such as crushed limestone particles, very fine chaff, straw in medium and large sizes, small stones, grog (sometimes even larger re-fired sherds), and various amounts of quartz. The fabric variants include Nile B2, B3, B4 and Nile B2/C1 (which is a coars-

321 Holthoer 1977, 86-88, pl. 18 assumed they were used for beer because they were most frequently found together with conical vessels, which he interpreted as bread moulds (the so-called ,flower $\operatorname{pot}^{\star}$ ). This interpretation continues to be challenged cf. recently Hubschmann 2010. Considering the shape criteria of certain forms this type of closed vessel cannot be called a 'bottle' because it has no narrow neck. er variant of Nile B2 with slightly coarser organic inclusions) and Nile D. Sometimes the coarseness of the inclusions gets to a point where the fabric has to be called Nile $\mathrm{C}$ but such cases are rare.

The wide variety in the raw material of these jars indicates that several large workshops were providing the vessels for the deliveries to the temple (of whatever kind of commodity). It is also possible that these differences represent various areas in a very large workshop. In some cases one gets the impression that everything that was lying about on the workshop floor found its way into the fabric. ${ }^{322}$

One of the most puzzling circumstances of the vast amounts of 'BB jars' coming out of the ground in the excavation at the temple is the fact that a considerable number of the bases were pierced and exhibit often quite large pre-firing holes (see also the base typology below). Thus, the probable raison d'etre, namely the holding of a

\footnotetext{
322 A visit to the largely still traditional pottery workshops at Hardassa north of Luxor exemplified this impression. In one of the open air workshops a lot of various debris and refuse was scattered all over the floor, whilst the other was kept extremely tidy. Interestingly the proprietor of the latter is a woman.
} 
liquid (even if not 'beer'), is made impossible for a considerable proportion of these vessels. At the same time a smaller number of vessels were accidentally cut at the base so that no liquid commodity would remain long within the vessel. Nevertheless, even these vessels were delivered to the tem- ple to be used. Hitherto, no reasonable conclusion was found to explain this phenomenon except to suspect votive offerings without actual commodities (see below).

The base typology (Type A to E) will be explained first, because the shape of the bases a)
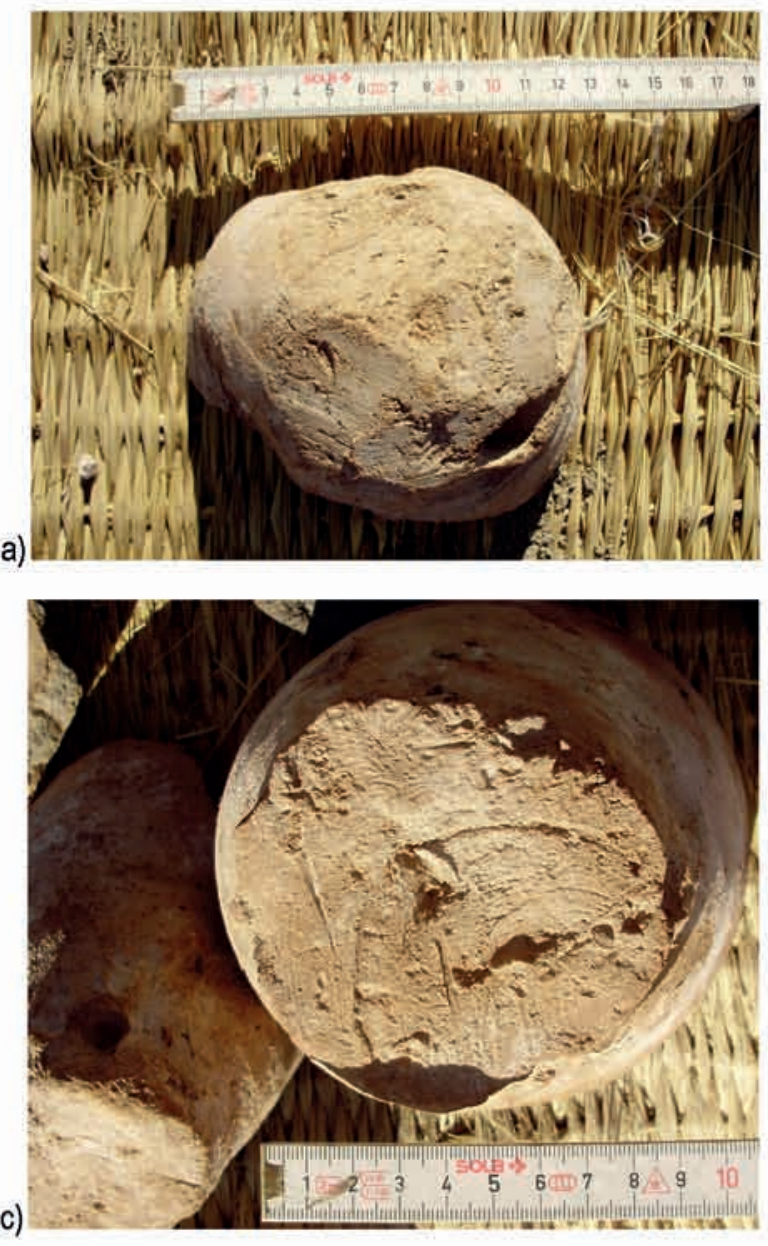
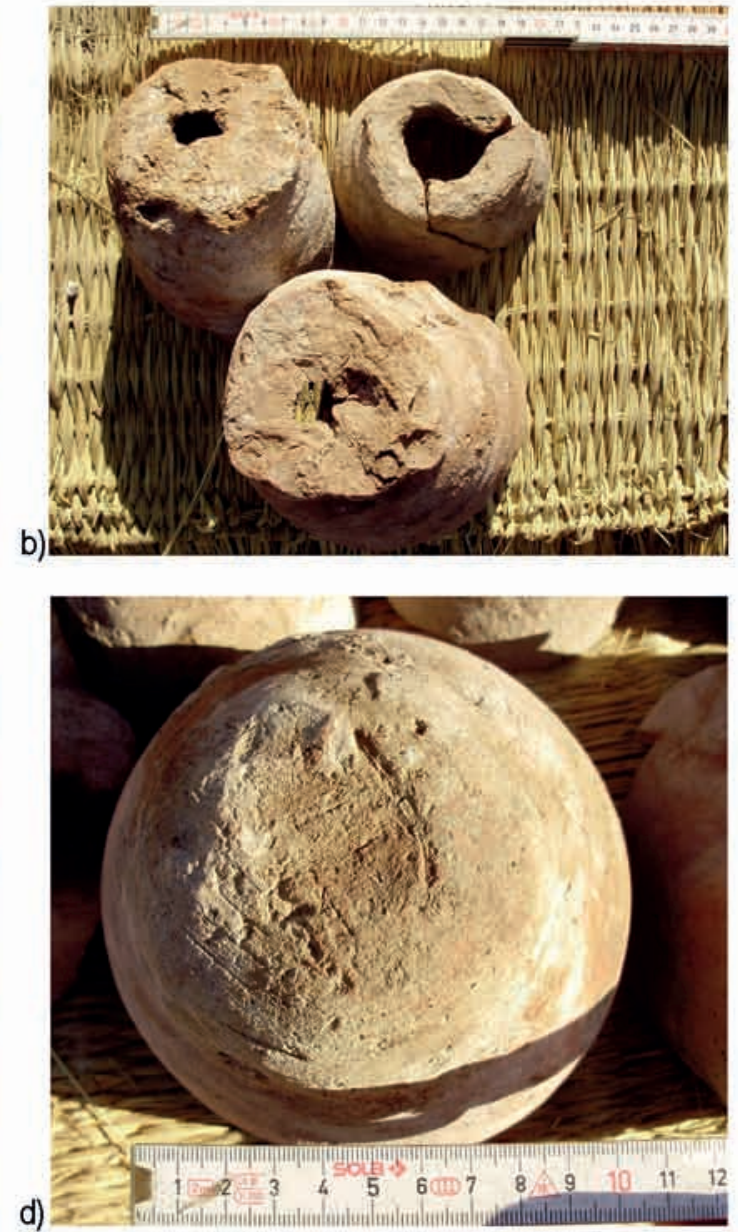

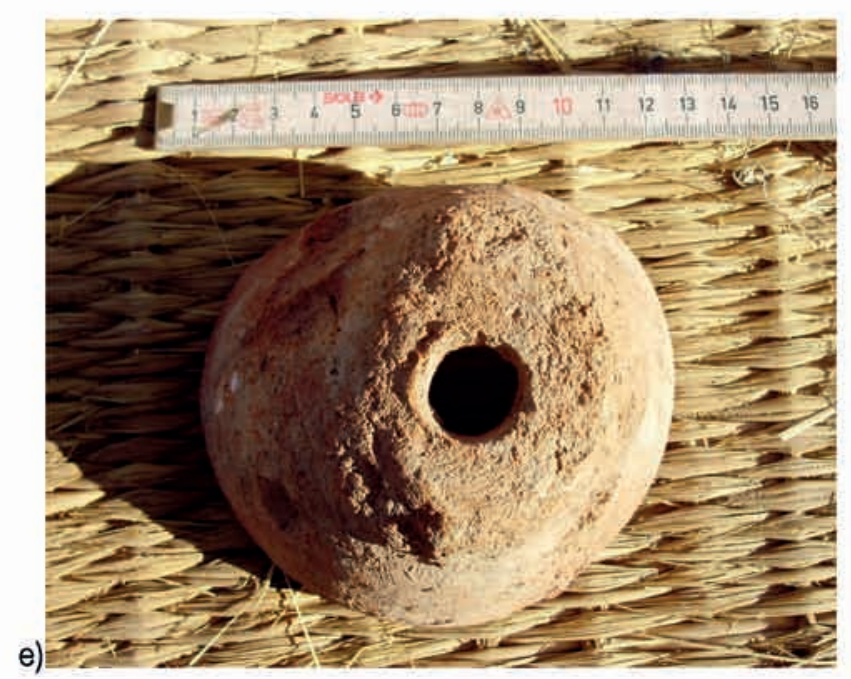

Fig. 44 Base typology of BB jars, New Kingdom, photos by B. Bader. 
directly influences the properties, the overall proportions and shapes of the complete profiles of the 'BB jars', which will be discussed below. ${ }^{323}$

\section{Type A (Fig. 44.a)}

Base Type A has been cut around the original edges of the 'BB jar' to such an extent that these vessels are not stable on a flat surface (they would be able to stand up only on sand). ${ }^{324}$ No variation of this type with a hole has been observed so far. This type is not represented in HoLthoer's typology.

Type B (Fig. 44.b)

Base Type B is represented by 'BB jars' that were cut from the wheel with string or wire (a hump is perhaps less likely for a base diameter of around 8.0 to $10.0 \mathrm{~cm}$ ) lifted off by hand leaving finger prints around the edges of the base. Finally the base was pierced to create a hole roughly in the centre of the base usually from the outside to the inside. Holthoer does not seem to have recorded holes within his material or there were none.

\section{Type C (Fig. 44.c)}

Base Type $\mathrm{C}$ corresponds to 'BB jars' cut from the wheel with string or wire and lifted from the wheel with the hands leaving finger imprints along the edges of the base. Seemingly no further steps were taken to make the vessels more appealing. ${ }^{225}$ In HolthoER's typology likely parallels are BB2 transitional 185/511:137, 185/217:1 and BB 3 simple $350 / 20: 1 .^{326}$

Type D (Fig. 44.d)

Base Type D can be differentiated by the fact that the edges of the base were cut away after the steps had been taken described under type C. This leads most of the time to the removal of the finger impressions around the base visible in type B and C. An irregular 'base' remains that is often rough-

323 A similar definition of the bases was undertaken for the material from the late $18^{\text {th }}$ Dynasty Saqqara burial of Horemheb, although no intentional holes seem to have occurred there. Cf. Aston 2011a, 219. Note that no examples with direct incurved rim without neck and shoulder exist there. The latest type III.4 in fig. VI.11 dates to the second half of the $19^{\text {th }}$ Dynasty and shows a sharp indentation and slight thickening of vessel wall immediately below the rim. The so-called pointed bases of Saqqara differ from the Theban type A in that there is no neat trimming below a horizontal edge. Unfortunately more ly triangular. The vessel stands up on flat surfaces. ${ }^{327}$ The vessel no. 185/419:1 from Holthoer's typology (BB2 transitional) seems to be similar to this base variant. ${ }^{328}$

\section{Type E (Fig. 44.e)}

Base Type E, finally, is the same as Type D except that a pre-firing hole was pierced in the centre of the base. Again Holthoer does not seem to have recorded holes in the bases or there were none in his material.

Complete 'BB jars' in the temple (Fig. 7, 36. $\mathrm{a}-\mathrm{b}, 41 . \mathrm{k}, 45)$

The overwhelming majority of BB jars in the temple and from the levelling layers/dumps to the north of the northern enclosure wall belong to the variety without shoulder and direct incurved rim. Often the jars survived the dumping intact (Fig. 51.c) and a high number of these jars survive with complete profile. Whilst not every variation can be discussed here, it seems that in 'BB jars' with Base type $\mathrm{B}$ and $\mathrm{C}$ the proportion of height to base and rim diameters is very similar overall and does not vary much although the careless manufacture often leads to extremely asymmetrical vessels. In many examples, admittedly often without good stratigraphic position, the base diameter is around $6.0-9.5 \mathrm{~cm}$ with a rim diameter of about 7.0 to $11.0 \mathrm{~cm}$. The position of the maximum diameter is usually at the mid height of the vessel. Maximum diameters are typically from 14.5 to ca $19.6 \mathrm{~cm}$, whilst the height varies from 20.0 to $29.5 \mathrm{~cm}$ with the middle of this range occurring most frequently.

The proportion of the other base types is of course different, due to the much smaller (or not existing) base diameter although the position of the maximum diameter is still in mid height of the vessel. Only a few vessels hitherto were discovered with the maximum diameter in the top third (Fig. 45). Still the rim is incurved and the vessel

detailed drawings of the 100s of BB jar bases from Saqqara as well as the exact measurements for base diameters remain unpublished. The range of base diameter varies from 6.0 to $9.0 \mathrm{~cm}$ and most seem to have belonged to our type $\mathrm{C}$ string cut, with edges and finger indents.

324 See examples in BADER 2015, 304-305, fig.4.20-21.

325 A classic example in BADER 2015, fig. 3.16.

326 Holthoer 1977, pl. 18.

327 BADER 2015, fig. 3.17-19.

328 Holthoer 1977, pl. 18. 


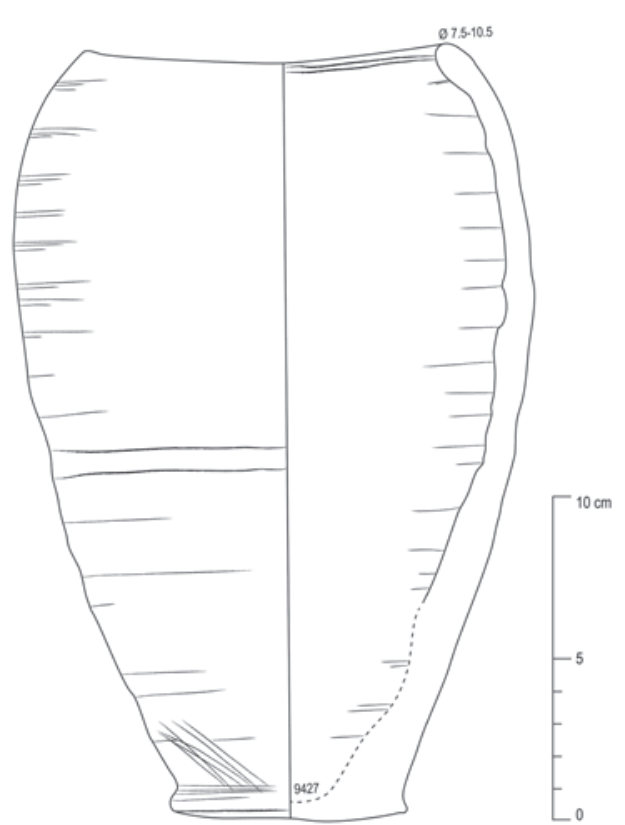

Fig. 45 BB jar, drawn by B. Bader, New Kingdom, inked by E. Schuster.

does not show a shoulder. Further studies need to confirm whether this difference in shape is due to chronological development.

No. 9427 (Enclosure Wall South: 940-South 4-L10), rd.= 10.5-7.5, max.d.= 15.8, bd.=6.5-7.0, ht.= 24.0, Nile B2/B3, section not visible, surface uncoated (10R6/3 pale brown), burnt, warped, wheelmade, base (type C) string cut, intact, weight $1.25 \mathrm{~kg}$, one kiln stain in mid height of the vessel.

'BB jars' with shoulder (Holthoer type 4) ${ }^{329}$ are exceedingly rare and only occur so far in a few rim fragments all over the site (see Fig. 23.h, 36.c). Whether this represents a chronological difference or a functional one needs to be investigated further. Interestingly Holthoer's subtype BB 2, which consists of several different profiles, was found to date from the mid- $18^{\text {th }}$ to the late $18^{\text {th }}$ Dynasty. ${ }^{330}$ Thus, the jars need to be studied in greater detail in order to find out, whether there are subtle developments that are chronologically sensitive.

\subsection{1.) A preliminary quantification of 'BB-jar' bases and base typology}

All base fragments from R4/5L2 (layer over the mouth of the shaft of Tomb XI, see above) were recorded and a quantitative analysis can be pre-

\footnotetext{
329 HOLthoer 1977, pl. 18.

330 SÄVE-SÖDERBERGH/Troy 1991, 33, 233, 238.

331 See plan in Seco Álvarez 2015, plan 1.
}

sented. Once this exercise is done for all contexts a comparative study of the distribution of these bases may be undertaken and similarities and differences will allow interpretation of this data.

The levelling and dumping layer continued along the entire North Enclosure Wall, more of which was discovered in squares R6, 930-940-North 5 and 910-920-North 5, ${ }^{331}$ and it should be noted here that the quantity of rims preserved is notably smaller than that of the bases. The reason for this differential preservation presumably lies in the fact that the bases are almost indestructible, whereas the rims are frail and prone to break easily. All rims were direct, incurved and without formal 'shoulder'. The tops of the rims vary in morphology: they may be trimmed, thickened or thinning.

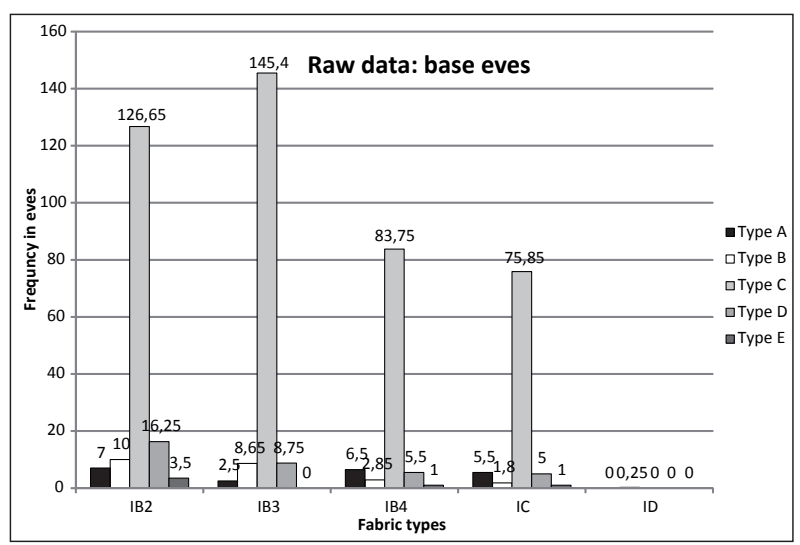

Fig. 46 Frequency distribution of BB jar base types.

Altogether 517.7 base estimated vessel equivalent $^{332}$ were recorded from layer 2. This would equate a minimum of 518 complete vessels. By far the most frequent base type is Type $\mathrm{C}-\mathrm{a}$ string cut base with clearly articulated edges around the base (see Fig. 44.c and frequency distribution chart Fig. 46). This holds true in all fabric variants. Type $\mathrm{E}$ is not much represented as almost all the examples assigned to this group show an accidental perforation, unwanted presumably. It occurred due to the fact that the base was cut off the wheel too thinly and subsequently the actual base was opened. Nevertheless, this mistake in production does not seem to have deterred the use of these vessels because these vessels were still fired $^{333}$ and delivered to the temple, perhaps as votive offerings.

\footnotetext{
332 ORTON et al. 1993, 21, 171-173.

333 Cf. Aston 2011a, 219, for the same observation in the tomb of Horemheb. For Mut el-Kharab Hubschmann 2010, 61.
} 


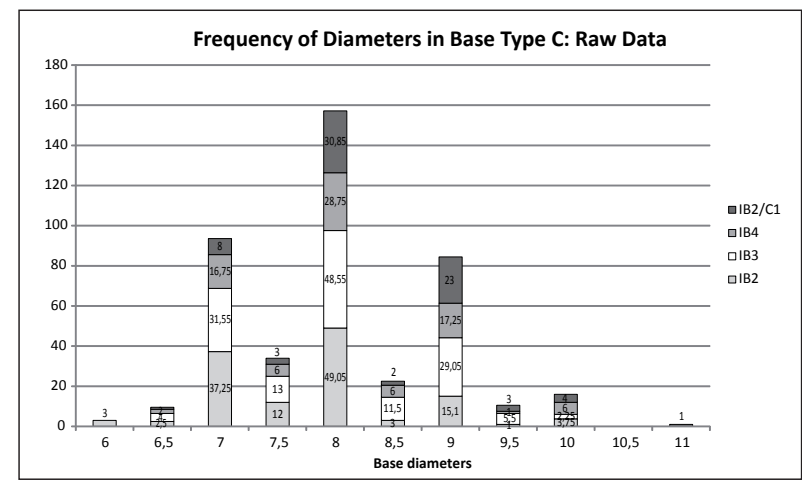

Fig. 47 Frequency distribution of base diameters of BB jars.

The frequency of 'BB jar' bases with intentional holes (Types B and E, see Fig. 46) is small compared to those without. This is in stark contrast to vessels of the same type group but belonging to a later sub-type from beneath the foundations of a Third Intermediate Period temple at Mut el-Kharab in the Dakhla Oasis. ${ }^{334}$ Thus, it is clear that the purpose of the vessels (with and without perforations) is most probably connected to offerings in temples and tombs. Hubschmann proposed a rather symbolical than practical purpose of these jars for offerings. On the other hand to assume that a considerable portion of these tens of 1000 s of vessels were delivered to the temple exclusively for symbolic actions containing nothing seems also unlikely considering the practice of the 'circulation of offerings' ('Opferumlauf'). Another possibility is the ritual of 'killing' pottery although the vessel type does not fit with the pictorial evidence. ${ }^{335}$

Further, the quantification of R4/5 layer 2 near the temple of Thutmosis III exemplifies that the function of these vessels needs not necessarily be the same in all contexts and periods. Base Type D (see Fig. 44.d) is next in terms of frequency although it falls far behind, whilst Types A and B are rare. Considering the frequency of fabrics Nile B3 is most common, whilst Nile B2 is next, followed by Nile B4 and Nile B2/C1. Nile D is only represented by one fragment. Interestingly within Base Type D, Nile B2 is the most frequently used fabric, with Nile B3 and B4 following closely. In the frequency distribution of base diameters $8.0 \mathrm{~cm}$ is most frequent in Type $\mathrm{C}$ closely followed by 7.0 and $9.0 \mathrm{~cm}$. It must be remembered, though,

\footnotetext{
334 Hubschmann 2010, 61.

335 SeILER 2010, 49-52.

336 Aston 2011b, 72.
}

that the bases of 'BB jars' are always uneven (see Fig. 44), thus it is probably safest to scrutinise the range between 7.0 and $9.0 \mathrm{~cm}$ as the most frequent range within this context with 7.5 and 8.5 as measurements in between. This result can be used as a basis for comparison with other areas. Also this result may serve as a beginning to address the assumption that the smaller the base diameter of the 'BB jars' the later in the chronological sequence the vessel should be dated. ${ }^{336}$

\section{3.) Painted pottery (Fig. 48)}

Among the pottery of the temple the occurrence of painted fragments can be called rare (more exact quantitative data needs to be collected to give percentages). Unfortunately most of the existing examples are from surface layers so that an independent chronological development cannot yet be sketched.

\subsection{1.) Monochrome red or black decoration}

Most frequently the red splash decoration occurs (see above) as well as red slipped pottery vessels with horizontal black line decoration, ${ }^{337}$ but small body fragments of closed vessels dominate with a few larger pieces. Black lines appear on vessels made of Nile clay fabrics as well as Marl clay fabrics (cf. Fig. 38.a). Another pattern encountered is black hanging elongated triangles usually decorating Marl A4 jars around the neck/shoulder transition. In terms of shape they seem to be similar to vessels decorated in bichrome style ${ }^{338}$ but no complete profile has yet been found.

\subsection{2.) Red and black decoration}

Red and black decoration on pottery is one of the hallmarks of Thutmosid ceramic production and thought to be inspired by Cypriote Bichrome ware. ${ }^{339}$ Whilst many sherds from the temple are decorated with alternating red and black horizontal lines few fragments allow a reconstruction of the overall shape of these vessels (Fig. 42.a, 48.a).

No. 20104, (square 960-North1-L1) Jar with horizontal lines, pres.ht. $=6.5$, Nile B2 inclusions but Marl Clay fabric look -mixed clay?, section beige core, pink oxidation zones, surface white

\footnotetext{
337 Aston 2003, fig. 1.b.

338 BADER 2015, fig. 5.26.

339 Aston 1998, 55.
} 
a)

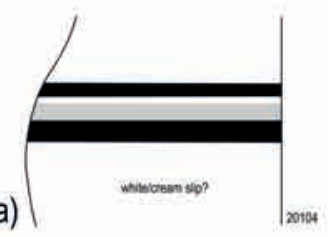

b)

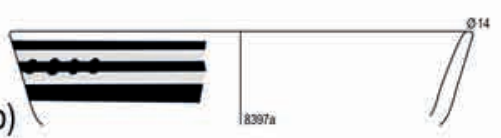

c)

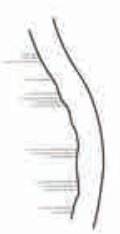

l)
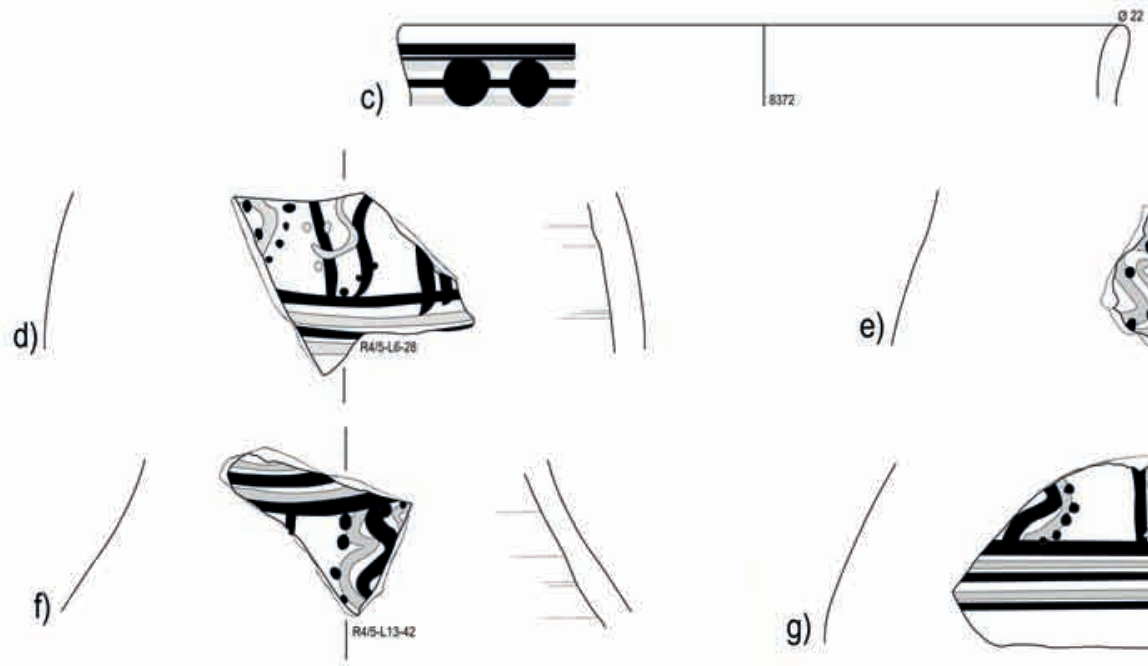

e)
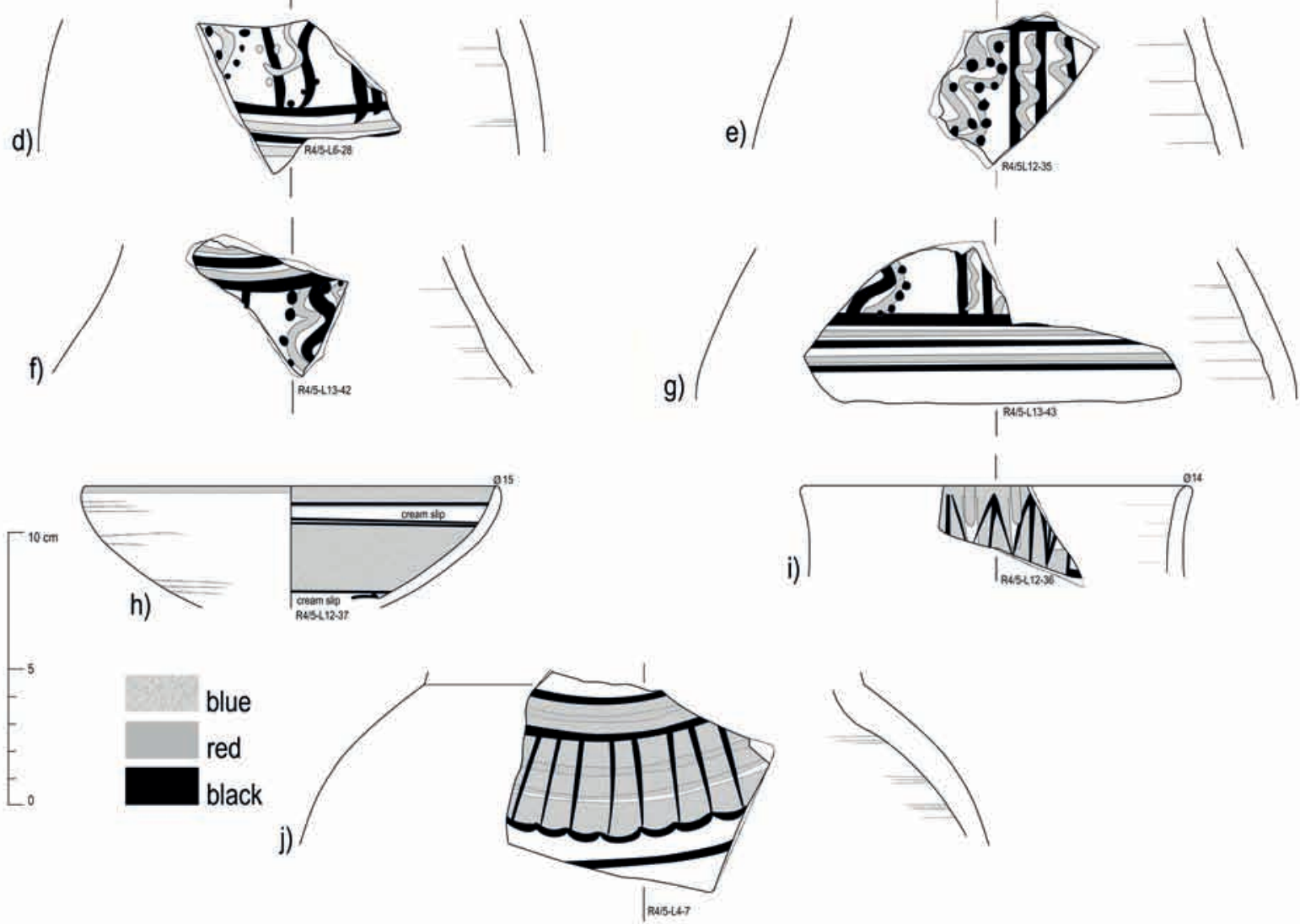

Fig. 48 Painted pottery, New Kingdom, drawn and inked by B. Bader.

slipped? (7.5YR8/3 pink), wheelmade, decorated with three horizontal lines, black-red-black, $8-10 \mathrm{~mm}$.

A common pattern well-known from other Thutmosid sites, ${ }^{340}$ is the combination of lines with dots ('swallow on a wire', dot-lines), which appear on open as well as on closed vessel shapes (see Fig. 38.b) and on Nile clay fabrics as well as on Marl clay fabrics. In addition there is a fabric that seems to be specific for this type of decoration: either a mixed clay fabric or a Nile clay body with Marl clay slip (Fig. 48.b-c). Other patterns observed but not yet recorded include closed ves- sels with horizontal black and red lines with vertical black lines,

No. 8397a (square 900-South 2-L8), open vessel, rd.= 14.0, pres.ht.= 2.9, Marl A2, section uniform light pink, surface uncoated (7.5YR7/4 pink), wheelmade, decorated with three horizontal lines, black-red-black 3-5 mm.

No. 8372 (square 900-South 2-L1), open vessel, rd. $=22.0$, pres.ht. $=2.5$, Marl + chaff?? mixed clay?, section uniform pink, surface uncoated (7.5YR7/3 pink), wheelmade, decorated with five horizontal lines, black -black -red -black -red and large dots on top of them.

340 RzEuSKa 2001. 
Another pattern observed is a combination of 'swallow on a wire' and hanging elongated triangles framed by a set of three black - red - black lines. $^{341}$

A hitherto unknown pattern variant combines wavy lines with straight lines and black and red dots (Fig. 48.d-g). This pattern is exclusively known from body sherds from contexts of Tomb XI, which were very much mixed in date. Neither the fabric, the pattern nor the manufacturing technology (W1) indicates a late date for these fragments. The chaine operatoire for the painting is as follows: 1) the cream slip ${ }^{342}$ was applied to the surface of the vessel, 2) the straight black lines were painted 3) the red wavy lines 4) the black wavy lines 5) the black dots. The pattern seems to consist of a panel with vertical straight and wavy lines with dots in red and black bordered between several vertical lines alternating black and red at the maximum diameter and the shoulder of the vessel.

R4/5-L6-28, pres.ht. $=4.9$, Nile B3, bichrome decoration on cream slip, wheelmade.

R4/5-L12-35, pres.ht.= 5.1, Nile B3, bichrome decoration on cream slip, wheelmade.

R4/5-L13-42, pres.ht. $=5.2$, Nile B3, bichrome decoration on cream slip, wheelmade.

R4/5-L13-43, pres.ht. $=5.7$, Nile B3, bichrome decoration on cream slip, wheelmade.

\subsection{3.) Red, black, blue and pink}

The study of the blue painted pottery from the temple has only just begun, because larger amounts of different blue painted sherds came mainly from the dumping/levelling layers to the north of the northern enclosure wall from the surface debris and the layers immediately below them. Thus, no stratigraphic order can be offered here.

Within the temple area blue painted pottery fragments also came only from some surface layers. So far only a few diagnostics were found in the fill of Tomb XI. The majority of the material consists of body sherds, which need more restoration work in order to find more joins and decoration schemes.

\section{Open Vessel (Fig. 48.h)}

$\mathrm{R} 4 / 5-\mathrm{L} 12-37$, rd. $=15.0$, pres.ht. $=4.6$, Nile B2, fine variant, cream slip with blue and black decoration

\footnotetext{
341 BADER 2015, Fig. 5.26.

342 The cream slip is not shown on the drawings in order not to obfuscate the patterns.
}

on interior, wheelmade; 1) cream slip, 2) black lines 3) blue bands.

\section{Closed Vessels (Fig. 48.i-j)}

R4/5-L12-36, rd.= 14.0, pres.ht. $=4.6$, Nile B3, cream slip with blue, red and black decoration on exterior, wheelmade; 1) cream slip, 2) black lines 3 ) red lines 4) blue petals and bands.

R4/5-L4-7, pres.ht. $=6.5$, Nile B3, cream slip with blue, red and black decoration on exterior, wheelmade; 1) cream slip, 2) red lines 3) blue bands 4) Black lines and petals.

Only few sherds found in the current excavations appear to date before the style dominant at the palace of Amenhotep III at Malqata (Fig. 49), ${ }^{343}$ but more recording is necessary to corroborate this hypothesis.

No. 15538, pres.ht. $=6.5$, Marl A4, section uniform greenish grey, surface uncoated $(2.5 \mathrm{Y} 8 / 4$ pale yellow), wheelmade, very well made, frieze of five horizontal black and blue lines; below a stem of a large lotus flower with two blossoms to the left and right.

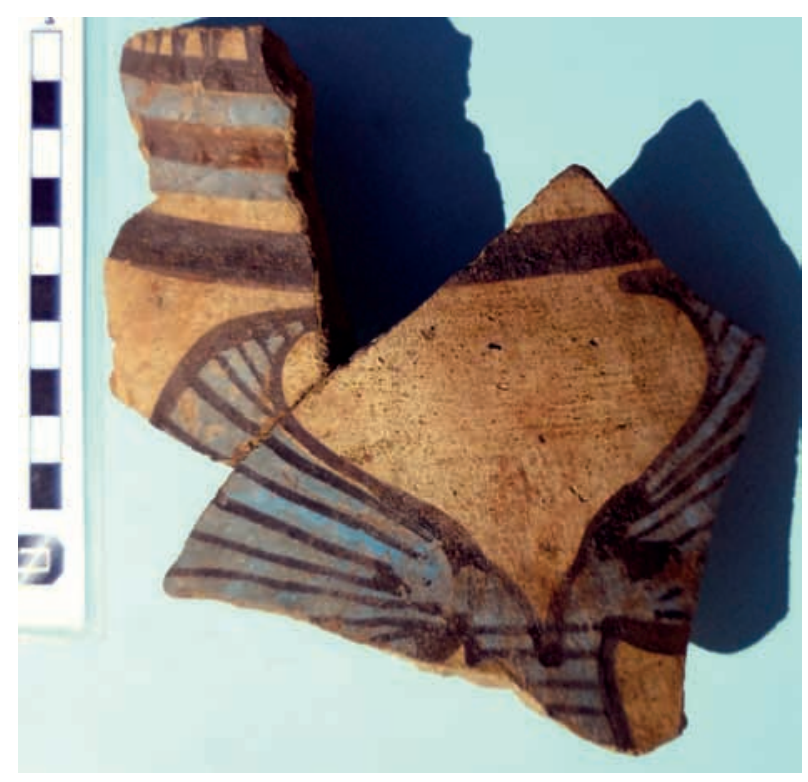

Fig. 49 Blue painted pottery, New Kingdom, photo B. Bader.

\section{8.) The pottery from later use of the temple - the Ramesside complex (Fig. 50.a)}

Already Grébaut recognised some $19^{\text {th }}$ and $20^{\text {th }}$ Dynasty remains in the northern part of the

\footnotetext{
343 Hope $1987 b$.
} 


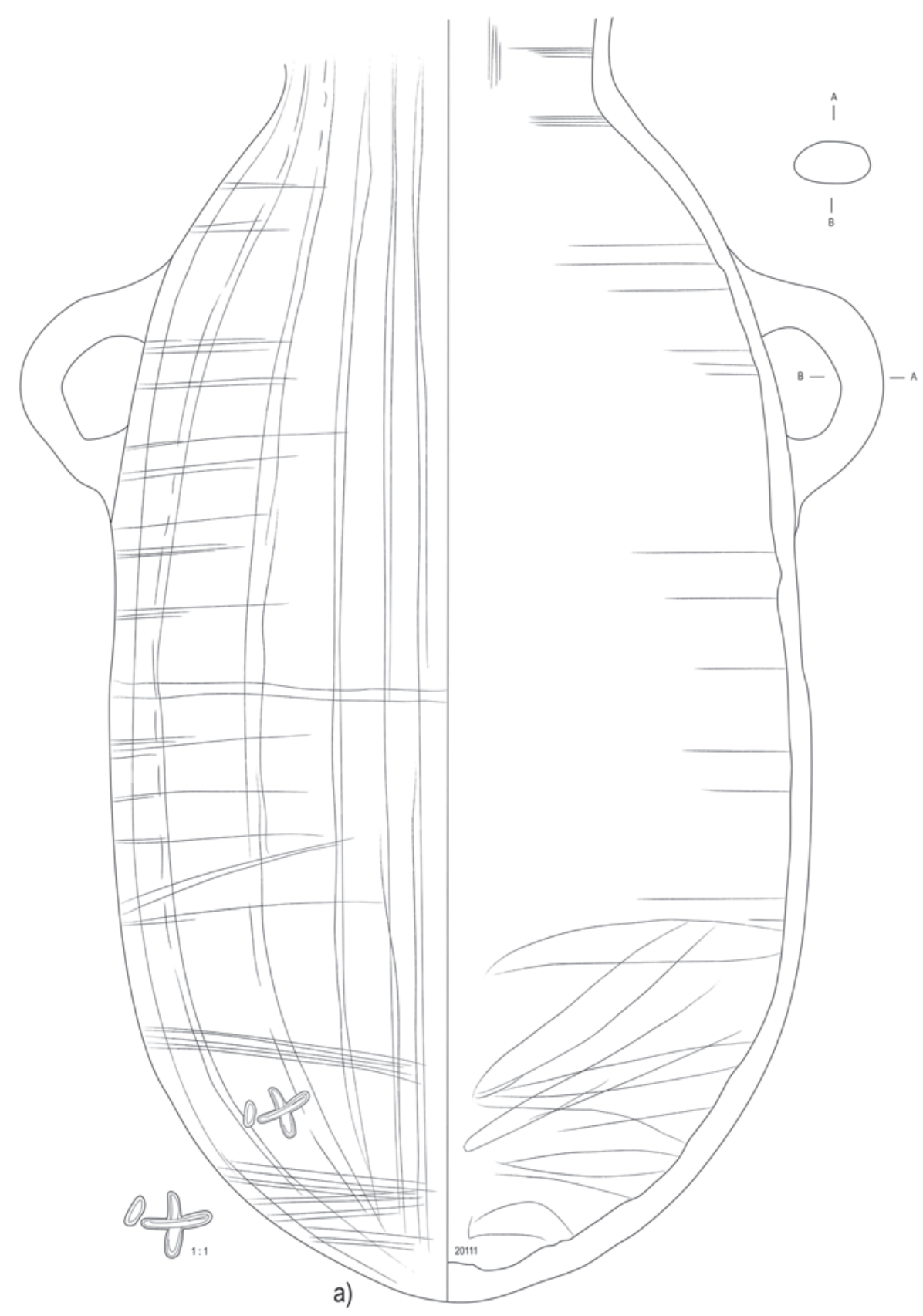

b)
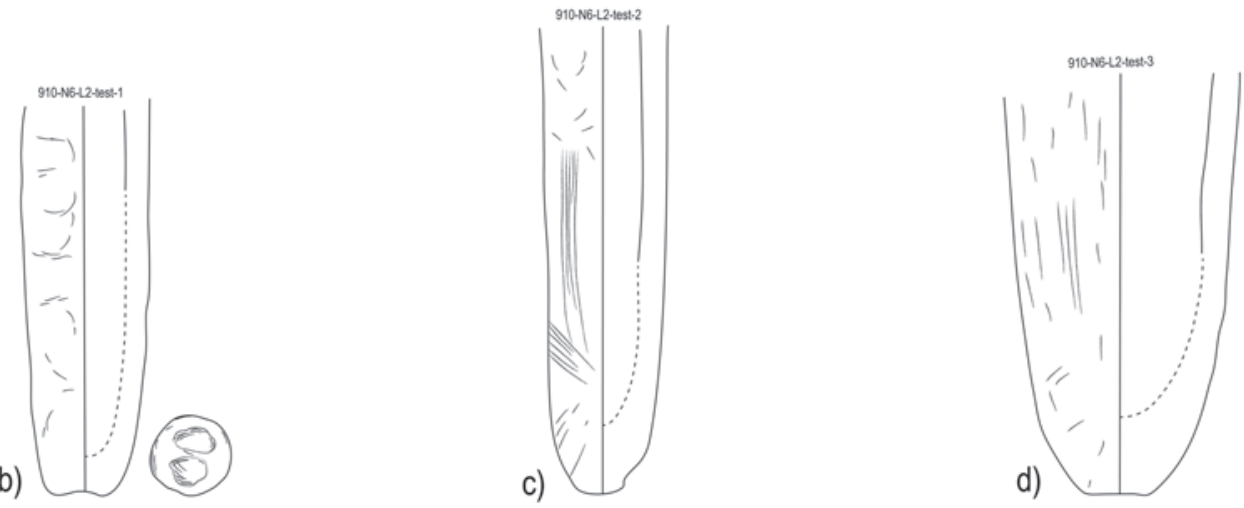

Fig. 50 a) Ramesside pottery. b-d) New Kingdom bread moulds, drawn by B. Bader, inked by E. Schuster. 
'magazines' as he took back to Cairo a 'zir' and registered it in the journal d'entrée, as no. 28916. Unfortunately he did not describe the pottery vessels in detail "[...]Dans un angle de la piece $F$ un vase en terre poreuse, analogue au zir arabe, mais plus petit, était fiché en terre; en $G$ j'ai trouvé l'ostracon signalé plus haut. Un peu partout gisaient de petits vases en poterie commune. Rien de bien caractéristique ne vient aider à déterminer à quelle époque ont été élevées ces construction; il me paraît cependant qu'on peut les faire remonter jusqu'à la XIXe ou XXe dynastie.[...]”344

In the north eastern part of the second court, a suite of rooms with a stone lintel of Ramesses II was found. ${ }^{345}$ The pottery of this area is in the process of being analysed and the first results can be presented here.

The topsoil contexts ${ }^{346}$ from 910-North 4-L1 and 910-North 3-L1 were heterogeneous and contained pottery from the Middle Kingdom, the Thutmosid and Ramesside period, possibly the Ptolemaic period as well as modern material (ballâs jar fragments ${ }^{347}$ ). During processing for the first time the late New Kingdom Marl D pottery repertoire appeared including fragments of amphorae and funnel neck jars as well as a few meat jar rims. Also interesting is the top of a large Marl D pilgrim flask (920-North 1-L2). ${ }^{348}$

'BB jar' bases and rims are still prominent in all the contexts, but none could yet be reconstructed to a complete profile. They still show the direct incurved rim, with only a few shouldered examples. The base diameters appear to be on average smaller than before, but hard data needs to be collected in order to corroborate the impression. Another fairly frequent vessel type is the Nile B dishes/bowls with strong rilling on the exterior of the base and almost S-shaped direct rims. ${ }^{349} \mathrm{~A}$ few blue painted sherds also appear out of context in the upper layers.

It seems as if a stratigraphic sequence starts from the Ramesside layers down to the Thutmosid levels perhaps encompassing the original magazines. The analysis of the material will show whether any of the original material stored there was still present, which must be deemed unlikely,

\footnotetext{
344 DARRESSY 1926, 15, note 3.

345 Seco Álvarez/Martinez Babon 2015d, Taf. 48 for the plan.

346 Note that these areas were excavated before, cf. note 6.

347 As the Egyptian workers still use ballalis for their water storage these sherds might be very recent.

348 Aston 1998, 462-463, cat.no 1692.
}

or if the magazines had been thoroughly cleaned out before being reused.

Pottery no. 20111 from context 920-North 4-L2 exemplifies the fact that the activities within the Ramesside complex continued at least into the late Ramesside period ( $20^{\text {th }}$ Dynasty). ${ }^{350}$

No. 20111, max.d.= 28.0, pres.ht.= 51.1, Marl D, section uniform reddish brown, surface covered with scum (5Y7/3 pale yellow), in some places 5YR7/6 reddish yellow, wheelmade, joining line visible - made in parts, base mould made, strong rilling lines seem to form a pattern with vertical burnishing, pre-firing pot mark (just in slip?).

\section{9.) The dumps to the north of the temple (squares 900 to 950-North 4-6)}

Since 2013 the northern side of the enclosure wall has been excavated and provided literally several tons of ceramic material for processing and analysis. In each season 2013, 2014 and 2015 over $1000 \mathrm{~kg}$ of pottery were processed. ${ }^{351}$ This assemblage was heavily dominated by 'BB jars' and their fragments as expected. In order to obtain a better idea of the features of the overall assemblage present in this area and its dating a 1.0 by $1.0 \mathrm{~m}$ test trench was sunk from just below the topsoil (Surface debris and L1) down to the bed rock in the 10.0 by $10.0 \mathrm{~m}$ square 910 -North 6 . All the ceramic material was collected for analysis. The excavation of this test trench provides the unique means for dating and quantification, which would otherwise be impossible due to the sheer bulk of the material.

\section{1.) The pottery test trench (910-North 6-L2-test)}

As indicated by the formerly collected ceramic material a very large component was formed by BB jars, even intact ones, although other vessel types were also found, such as shouldered jars with long neck, slender elongated jars, large vessels with vertical scraping (see Fig. 52.e-f, 53a) ${ }^{352}$, large white slipped handmade storage jars (cf. Fig. 37.e), a variety of dishes and bowls (including ring pattern burnishing), model dishes and bowls, oval

\footnotetext{
349 Aston 1998, 326-331, for a variety of morphological traits and surface treatments.

350 See Aston 1996, 66, Group 49 and fig. 203c-205d.

351 Bettina Bader would like to thank D. Penzer for her indispensable help in 2015.

352 BADER 2015, fig. 6.25.
} 
a)
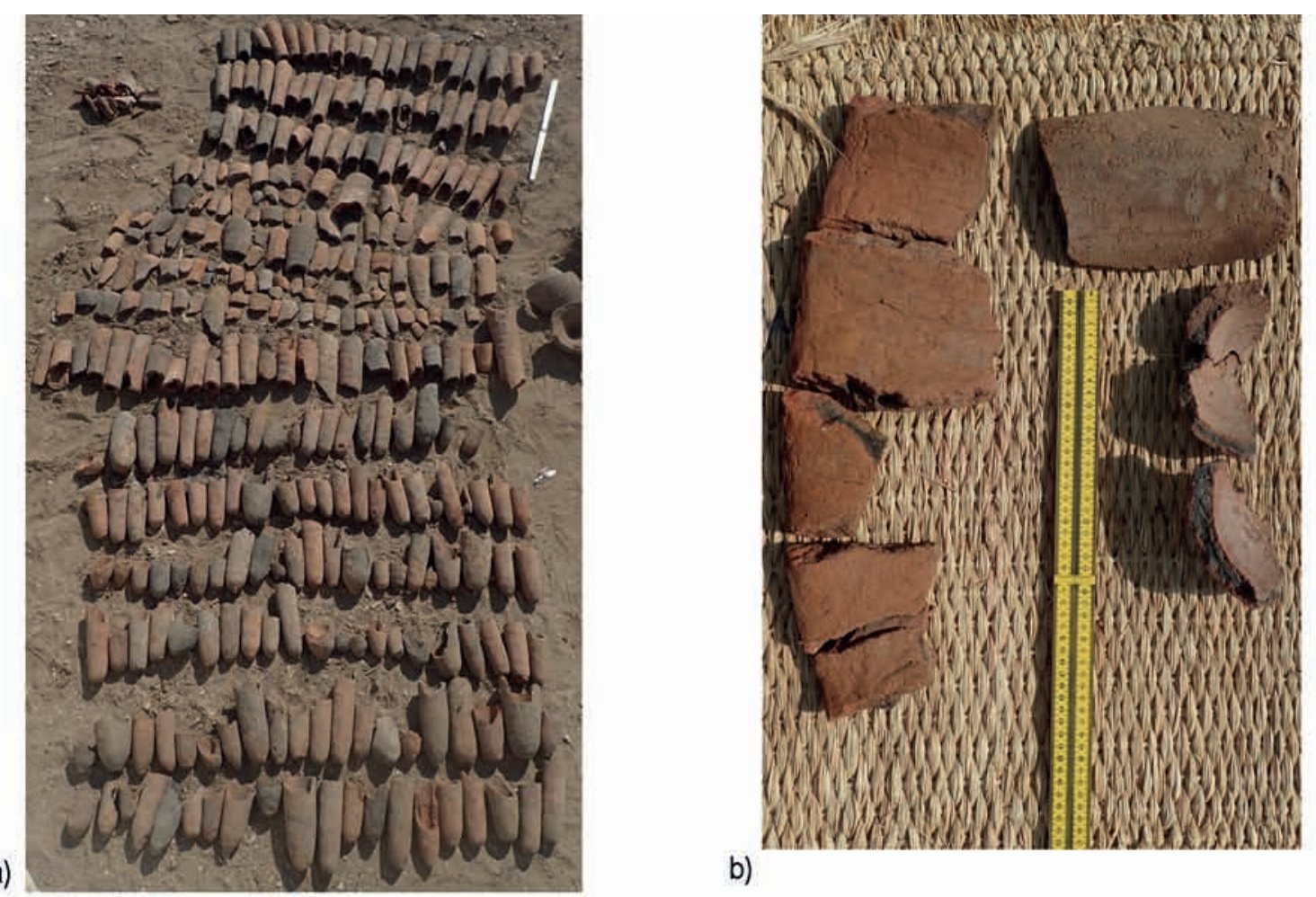

b)

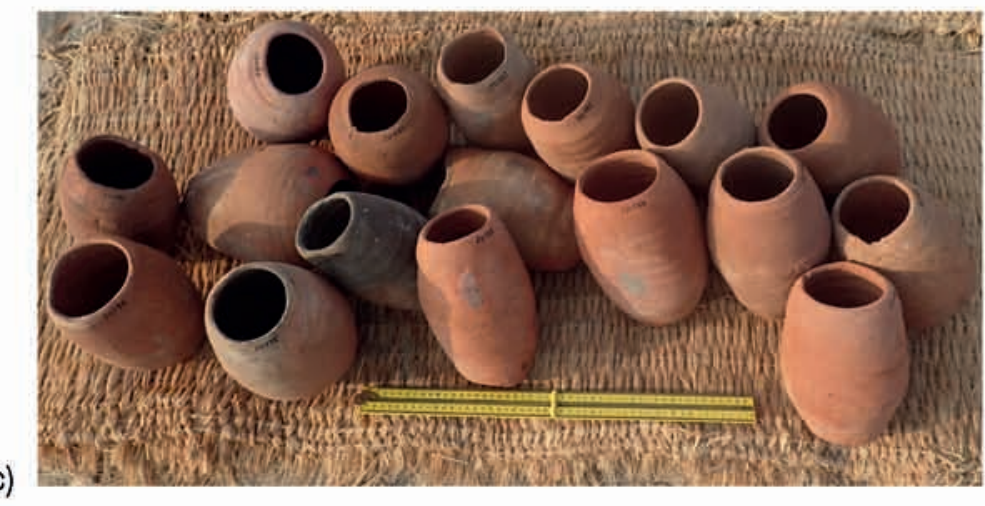

Fig. 51 Pottery from the test trench, photos by B. Bader.

trays (cf. Fig. 36.g), burners (cf. Fig. 52.a), narrow bread mould fragments and a varying number of blue painted vessel fragments.

The most surprising find within the material from test trench 910-N6 was the large amount of bread moulds hitherto underestimated because they were rarely preserved in larger pieces (see Fig. 51.a). The material consisted of Nile B2 or the slightly rougher Nile B2/C1 with diameters for the rims ranging from 8.0 to $4.0 \mathrm{~cm}$ (most of them were preserved to a $100 \%$ circle). The base types occurring are pointed, with two finger impressions on the base or a flattened base (Fig. 50.b-d). This material shows that in contrast to common opinion not all New Kingdom bread moulds are narrow, and there were even oversized and huge bread moulds (Fig. 51.b).

910-N6-L2-test-1, bd. $=2.3$, pres.ht. $=12.3$, Nile $\mathrm{B} 2 / \mathrm{C} 1$, surface uncoated, handmade over core, two pre-firing finger impressions on base.

910-N6-L2-test-2, pres.ht.= 14.4, Nile B2, surface uncoated, handmade over core, pointed base, cream of clay still present inside.

910-N6-L2-test-3, bd. $=2.1$, pres.ht. $=13.0$, Nile $\mathrm{B} 2 / \mathrm{C} 1$, surface uncoated, handmade over core, base flattened or with one pre-firing finger impression, cream of clay still present inside.

The recorded data will make it possible to quantify all the vessel types occurring including 'BB jars', large jars with vertical scraped base, 

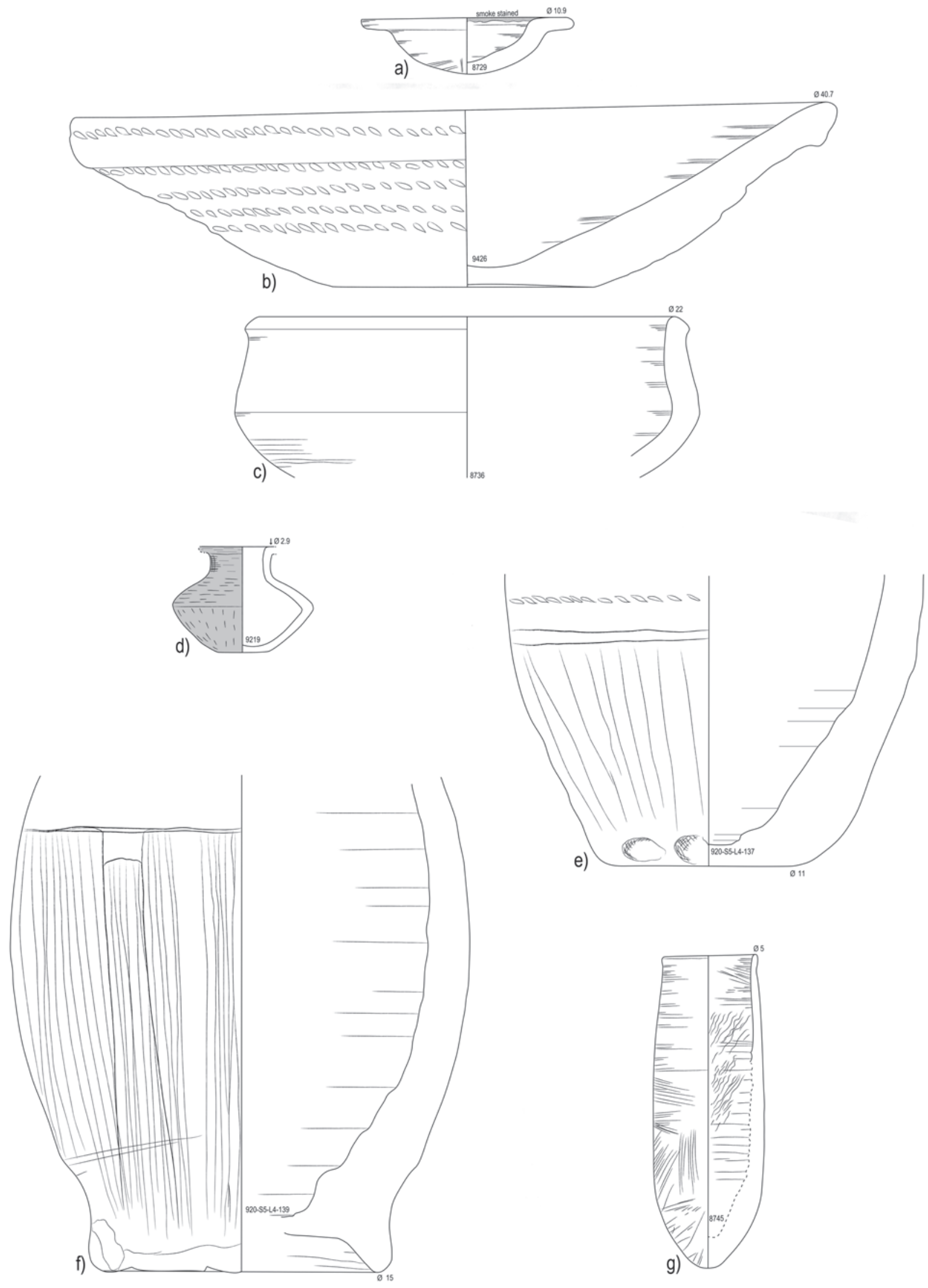

Fig. 52 New Kingdom pottery, drawn by B. Bader, inked by E. Schuster. 
burners, small beaker jars (one with a black rim band), dishes with red splash decoration, dishes with incurved rim and red rim band, carinated bowls with red rim band, flower pots, model dishes, slender jars, one jar with white slip and black horizontal lines, some marl clay fabric jars and a few other types, that hint at a slightly later date towards the late $18^{\text {th }}$ or $19^{\text {th }}$ Dynasty (funnel neck jars (Nile C with red slip on exterior)), ${ }^{353}$ and large necked storage jars with rolled or folded rim (Nile $\mathrm{B} 2 / \mathrm{C} 1),{ }^{354}$ for example. Interestingly no Marl D pottery was among the material processed and no blue painted fragments in this instance.

All material from test trench 910-North 6-L2-test was processed and a selection for further recording was made. The weight of the processed material from the trench alone amounts to $608 \mathrm{~kg}$. To this must be added the weight of the intact BB jars $(17)^{355}$ (Fig. 51.c) and that of the complete profiles (46), all in all 63 complete profiles.

\section{0.) Selected pottery types from the general site corpus of the temple}

A few interesting and/or more complete vessels are shown here in addition to the New Kingdom pottery discussed above. Often the complete profiles are from the surface and are dated to the early to mid- $18^{\text {th }}$ Dynasty only by parallels. Nevertheless it seems worthwhile to show vessel types that are perhaps not commonly known to occur in the temple of Thutmosis III. Note that a short overview was published before. ${ }^{356}$

\section{Nile B2 - Small burner (Fig. 52.a)}

Although a burner fragment has been shown already, this vessel type is also known from complete profiles in a variety of sizes (cf. Fig. 36.h). A number were found to the north of the temple and await recording.

No. 8729 (R6-L1), rd.= 10.9, ht.=3.1, Nile B2, section red core light brown oxidation zones, surface uncoated (7.5YR6/4 light brown), wheelmade, base trimmed with a tool, smoke stained on interior with resin remains.

\section{Nile C2 - Large bowl (Fig. 52.b)}

Large bowls such as this complete example are very difficult to date and occur during the entire

\footnotetext{
353 E.g. Aston 1998, 284-293.

354 Aston 1998, 306-307.

355 The average weight of an intact vessel is about $1.5 \mathrm{~kg}$.
}

New Kingdom. ${ }^{357}$ This piece is particularly interesting because it bears a number of traces and adaptations for daily use.

No. 9426 (940-S5-L3), rd.=40.0, bd.= 14.0, ht. $=$ 9.3-9.6, Nile C2, wide dark grey core, red and brown oxidation zones, surface uncoated, wheelmade, five rows of rope impressions on the exterior (the rope was turned in Z-direction), all around the rim in ca $5.0 \mathrm{~cm}$ width, the interior surface is smoke stained, the base was completely rubbed off by intention, presumably the dish had originally a ring base.

\section{Nile B2/C1 - Cooking pot (Fig. 52.c)}

Among all the hitherto recorded ceramic material this fragment is the first cooking pot so far encountered. Unfortunately from a surface layer, it is an important addition to the site corpus.

No. 8736 (910-S2-L1), rd.=22.0, pres.ht. $=8,5$, Nile B2/C1 (abundant quartz), section wide black core with red and brown oxidation zones), surface uncoated (5YR6/4 light reddish brown), wheelmade, smoke blackened and sooted on exterior.

\section{Nile B2 - Medium beaker jars (Fig. 52.g)}

The full profile of a very slender beaker provides the site corpus with a narrower variation with a more pointed base than similar beakers (Fig. 17.ef, 19.b, 27.b).

No. 8745 (930-S3-L2), rd.= 5.0, max.d. $=6.9$, $h t .=16.7$, Nile B2 fine variant, section violet core with red and brown oxidation zones, surface uncoated, wheelmade, base wheelmade and trimmed squeezed and turned in the upper part of the vessel.

\section{Nile clay fabric - Vessels with vertically scraped lower parts (Fig. 52.e-f, 53.a)}

Frequently during processing heavy bases made from various Nile clay fabrics with very rough vertical scraping in the lower part of the vessel were noticed. Until now two complete profiles could be reconstructed, which prove that a similar technology used for the bases, does not automatically mean that the same vessel types occurred. The two complete profiles published elsewhere ${ }^{358}$ represent presumably storage jars, but no. 8723 is slender with a narrow neck and the max.d. in the 

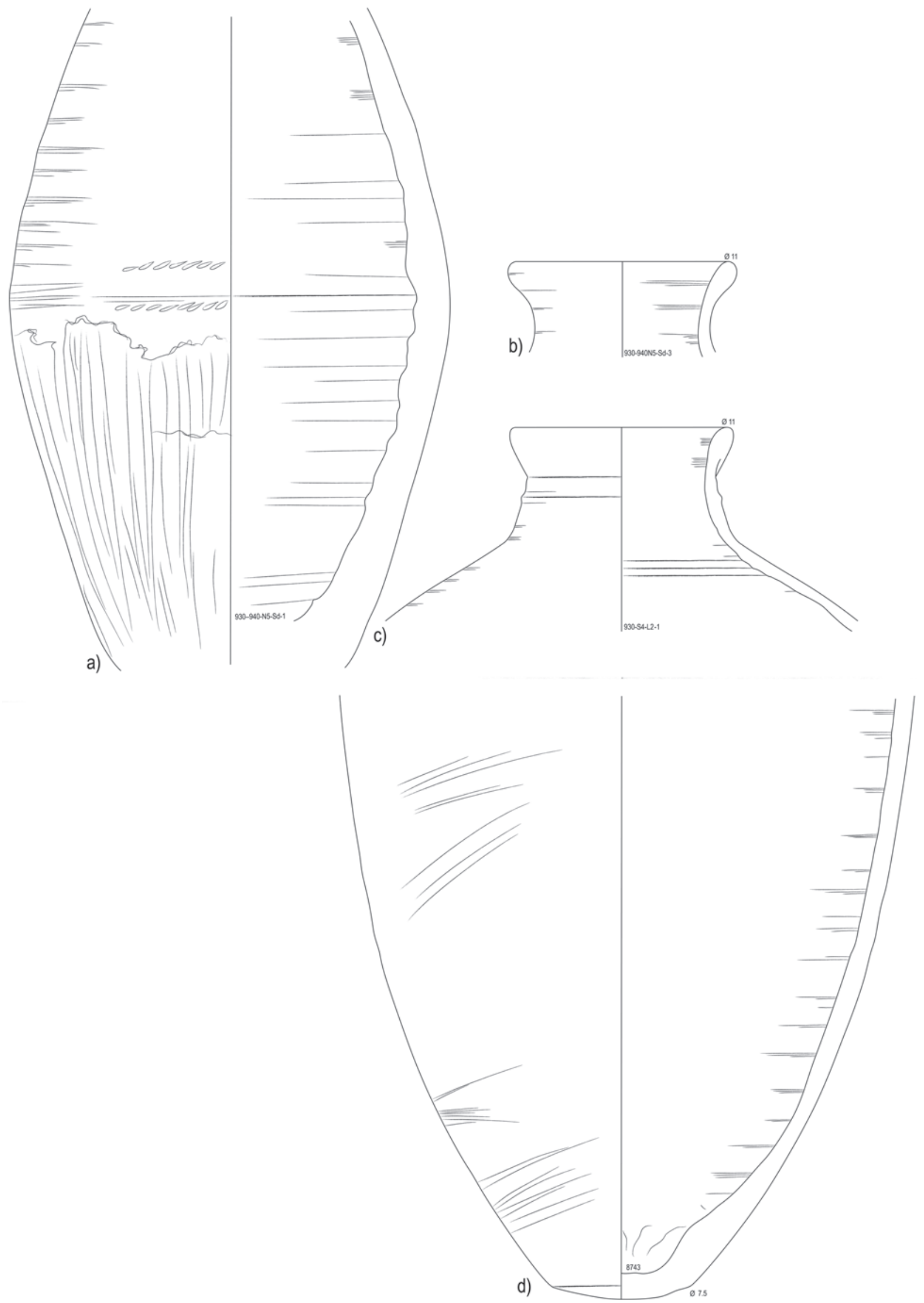

Fig. 53 New Kingdom pottery and Canaanite jars, drawn by B. Bader, inked by E. Schuster. 
lower third of the vessel, whilst no. 8724 is wide bodied with the max.d. in the top third. ${ }^{359}$ Interestingly also some of these large jars exhibit pre-firing holes in their bases, which casts some doubts on their actual use. The base and body fragments presented here are meant to demonstrate that several more types may be hidden among such bases. That they are contemporary with the temple of Thutmosis III is proven by the occurrence of just such a base in the tomb of Tjanuni. ${ }^{360}$ From Qau a large vessel, type $166,{ }^{361}$ with a height of approximately $50.0 \mathrm{~cm}$ and the same vertically scraped base technology is known. It must have been very rough, because in the British School of Archaeology pottery corpus drawings of the New Kingdom, the manufacturing technology is usually not shown. Also marked are the rope impressions around the maximum diameter. The burial groups in which this type was found suggest a date in the early to mid- $18^{\text {th }}$ Dynasty. Some of these accompanying types occur also at the temple of Thutmosis III.

920S5L4-137, bd. $=11.0$, pres.ht. $=15.5$, Nile C3, section dark grey core red and brown oxidation zones, surface uncoated, wheelmade (W1), base string cut (?) or formed on the floor with deep finger impression on the side, lower part of vessel very roughly scraped vertically on exterior.

920S5L4-139, bd. $=15.0$, pres.ht. $=26.3$, Nile $\mathrm{C} 3$, section wide dark grey core thin brown oxidation zone on exterior, surface uncoated, wheelmade (W1), ring base turned (?), lower part of vessel very roughly scraped vertically on exterior.

930-940-N5-Sd-1, pres.ht. $=36.0$, Nile B3, section wide grey core, thin red oxidation zones, surface uncoated, wheelmade (W1), lower part of vessel very roughly scraped vertically on exterior.

\section{Bibliography}

ARNOLD, Do.

1968 Keramikbeispiele aus den Gräbern der frühen 11. Dynastie von El-Târif, MDAIK 23, 38-67.

1972 Weiteres zur Keramik von el-Târif, MDAIK 28, 33-46.

1976 Wandbild und Scherbenbefund, MDAIK 32, 1-34.

359 It is very possible that this type is a more slender and taller continuation of one of Seiler's Theban style beer jars, that is flat based. SEILER 2010, fig. 4.3-4, although the scraping did not happen vertically in the $13^{\text {th }}$ Dynasty.
Marl A2 -Small squat jar (Fig. 52.d)

Small squat and carinated containers such as this form one of the hallmarks of the $18^{\text {th }}$ Dynasty. The heavy use traces around the rim suggest that this vessel was not just a symbolic offering.

No. 9219 (980-S3-L1), rd.= 2.9, bd.= 2.6, max.d. $=7.5$, ht. $=5.6$, Marl A2, section uniform light red, surface red slipped and burnished on exterior (horizontally on rim and shoulders; below the carination vertically), wheelmade (W1), base wheelmade and very well smoothed, the rim looks abraded all around, no original rim left.

\section{Imported amphorae (Fig. 53.b-d)}

The imported Canaanite material is scarce although almost every context contains a few body sherds. The methodological problem lies in the fact that until now most of the imported material comes from the surface (except that of the foundation deposit, see above). Thus, the classification of the material and the research into trade relations of the temple administration with the Levant remains to be studied in detail.

No. 8743 (910-S5-L5), bd.= 7.7, pres.ht= 32.8, FF (not classified yet), section dark grey core, thin red oxidation zone outside, surface uncoated (2.5Y7/3 pale yellow), wheelmade (W1-2), base mould made.

930-S4-L2-1, rd.= 11.0, pres.ht.=11.1, FF3, section dark brown with beige and red oxidation zones (Fig. 1.v), surface uncoated, wheelmade (W1-2).

930-940N5-Sd-3, rd.= 11.0, pres.ht. $=5.2, \mathrm{FF} 4$, section dark brown with beige and red oxidation zones (Fig. 1.w), surface uncoated covered with white scum, wheelmade (W1-2).

1981 Ägyptische Mergeltone (»Wüstentone«) und die Herkunft einer Mergeltonware des Mittleren Reiches aus der Gegend von Memphis, 161-191 in: ArNolD, D. (ed.) Studien zur altägyptischen Keramik, SDAIK, Mainz am Rhein.

1988 Pottery, 106-146 in: Arnold, D. (ed.) The Pyramid of Senwosret I Volume I, Publications of the Metropoli$\tan$ Museum of Art 22, New York.

\footnotetext{
360 Brack/Brack 1977, pl. 6.5, 23.

361 Brunton 1930, pl. 29.166.
} 
1991 Amenemhat I and the Early Twelth Dynasty at Thebes, Metropolitan Museum of Art Journal 26, $5-48$.

1993 Techniques and Traditions of Manufacture in the Pottery of ancient Egypt in: Arnold, D./Bourriau, J. (eds.), An Introduction to Ancient Egyptian Pottery, Sonderschrift des Deutschen Archäologischen Instituts Abteilung Kairo 17, Mainz am Rhein.

Aston, B. G.

1994 Ancient Egyptian Stone Vessels: Materials and Forms, SAGA 5, Heidelberg.

2011a The Pottery in: Raven, M. J. (ed.) The Memphite Tomb of Horemheb, Commander-in-chief of Tutankhamun V: The Forecourt and the Area south of the Tomb with some Notes on the Tomb of Tia, Turnhout.

Aston, D. A.

1989 Ancient Egyptian "Fire Dogs": A New Interpretation, MDAIK 45, 27-32.

1996 Egyptian Pottery of the Late New Kingdom and the Third Intermediate Period (Twelfth to Seventh Centuries $B C$ ), Studien zur Archäologie und Geschichte Altägyptens 13, Heidelberg.

1998 Die Keramik des Grabungsplatzes QI, Teil 1 Corpus of Fabrics, Wares and Shapes, Forschungen in der Ramsesstadt, Mainz am Rhein.

2003 New Kingdom Pottery Phases as Revealed by welldated Tomb Contexts, 135-162 in: BieTAK, M./CZERnY, E. (eds.), The Synchronisation of Civilisations in the Eastern Mediterranean in the Second Millennium B.C. II.

2004a Amphorae in New Kingdom Egypt, Ägypten und Levante 14, 175-214.

2004b Tell el-Dab'a XII. A Corpus of Late Middle Kingdom and Second Intermediate Period Pottery, UZK 20, Vienna.

2006 Making A Splash. Ceramic Decoration in the Reigns of Tuthmosis III and Amenophis II, 65-74 in: CZERNY, E./Hein， I./Hunger, H./Melmann， D./Schwab，A. (eds.), Timelines. Studies in Honour of Manfred Bietak, I, Leuven.

2008 The Pottery -Untersuchungen im Totentempel des Merenptah in Theben, Band IV, Beiträge zur ägyptischen Bauforschung und Altertumskunde 17, Mainz am Rhein.

2011b The Pottery from L49, 28-83 in: Perez Die, C. (ed.) Hercleópolis Magna (Ehnasya el Medina, Egipto), La Necrópolis 'real' del Tercer Período Intermedio y su reutilización, Madrid.

BADER, B.

2001 Tell el-Dabca XIII. Typologie und Chronologie der Mergel-C-Ton Keramik. Materialien zum Binnenhandel des Mittleren Reiches und der Zweiten Zwischenzeit, Untersuchungen der Zweigstelle Kario des Österreichischen Archäologischen Instituts 19, Vienna.
2007 A Tale of Two Cities: First Results of a Comparison between Avaris and Memphis, 249-268 in: BIETAK, M./Czerny, E. (eds.), The Synchronisation of Civilisations in the Eastern Mediterranean in the Second Millennium B.C., III, Proceedings of the SCIEM 2000 Euro Conference, Vienna, 28th of May to 1st of June 2003.

2009 Tell el-Dabca XIX. Auaris und Memphis im Mittleren Reich und in der Hyksoszeit. Vergleichsanalyse der materiellen Kultur, Untersuchungen der Zweigstelle Kario des Österreichischen Archäologischen Instituts 31, Vienna.

2010 Processing and Analysis of Ceramic Finds at the Egyptian Site of Tell el-Dab'a/Avaris, 209-233 in: Horejs, B./Jung, R./Pavúk, P. (eds.), Analysing Pottery. Processing - Classification - Publication, Studia Archaeologica et Medievalia 9, Bratislava.

2011 Vessels in Ceramics and Stone: The Problem of the Chicken and Egg?, 125-171 in: Aston, D. et. al., Under the Potter's Tree. Studies on Ancient Egypt Presented to Janine Bourriau on the Occasion of her 70th Birthday, Orientalia Lovaniensia Analecta, Leuven, Paris, Walpole, CT.

2015 Overview of Pottery occurring in the Temple of Millions of Years of Thutmose III, 293-309 in: SECO Álvarez, M./Jódar Minarro, A. (eds.), The Temples of Millions of Years in Thebes, Granada.

2016 Quantification as a Means of Functional Analysis: Settlement Pottery of the Late Middle Kingdom at Tell el-Dab a, 47-67 in: Bader, B./KNoblauch, C./ KöHLER, E. C. (eds.), Vienna 2 -Ancient Egyptian Ceramics in the 21st Century, Orientalia Lovaniensia Analecta, Leuven, Paris, Bristol, CT.

BIETAK, M.

1991 Tell el-Dabca V: Ein Friedhofsbezirk der Mittleren Bronzezeitkultur mit Totentempel und Siedlungsschichten, UZK 8, Vienna.

BOURriau, J.

2006 Technology in the Pottery of the Middle and New Kingdoms: An Underrated Tool in the Archaeologist's Armory, 31-43 in: Mathieu, B./Meeks, D./Wissa, M. (eds.), L'apport de l'Égypte à l'histoire des techniques. Methodes, chronologies et comparaisons, Bibliothèque d'Étude 142, Le Caire.

Bourriau, J./De Meyer, M./Op de Beeck, L./Vereecken, S.

2005 The Second Intermediate Period and Early New Kingdom in Dei al-Barsha, $\ddot{A} \& L$ 15, 101-129.

Bourriau, J./Gallorini, C.

2016 Survey of Memphis VIII, Kom Rabia: The Middle Kindom and Second Intermediate Period Pottery, EES Excavation Memoir, London.

Brack, A./Brack, A.

1977 Das Grab des Tjanuni-Theben nr. 74, Archäologische Veröffentlichungen 19, Mainz am Rhein. 
BRUNTON, G.

1930 Qau and Badari III, British School of Archaeology in Egypt, London.

BUDKA, J.

2006 The Oriental Institute Ahmose and Tetisheri Project at Abydos 2002-2004: The New Kingdom Pottery, Ä\&L 16, 83-120.

Carnarvon, G. H. t. E. o./Carter, H.

1912 Five Years' Explorations at Thebes, London/New York.

Consonni, A./Sesana, A.

2016 The Pottery from a Middle Kingdom Tomb at the Temple of Millions of Years of Amenhotep II, Thebes, 103-118 in: Bader, B./Knoblauch, C./Köhler, E. C. (eds.), Vienna 2: Ancient Egyptian Ceramics in the 21st Century, Vienna.

DARRESSY, G.

1926 Voyage d'inspection de M. Grebaut en 1889, ASAE 26, $1-22$.

DAVIS, T. M./CARTER, H.

1906 The Tomb of Hatshopsitu, London.

Dreyer, G./Hartung, U./Hikade, T./Köhler, E. C./Müller, V./Pumpenmeier, F.

1998 Umm el-Qaab, Nachuntersuchungen im frühzeitlichen Königsfriedhof, 9./10. Vorbericht, MDAIK 54, 77-167.

FAKHRY, A.

1937 Miscellanea, Annales du Service des Antiquités de l'Egypte 37, 25-38.

Guidotтi, M. C.

1985 La Ceramica della Tomba '79 a Gurna, Egitto e Vicino Oriente 8, 25-61.

Guidotti, M. C./Silvano, F.

2003 La Ceramica del Tempio di Thutmosis IV a Gurna, Pisa.

HeIN, I.

2001 Untersuchungen und vorläufige Bilanz zur Keramik aus 'Ezbet Helmi, speziell Areal H/V, Ägypten und Levante 11, 121-147.

2012 La céramique importée palestinienne du 2e millénaire av. J.-C. provenant de la fouille du Trésor de Thoutmosis Ier in: JacQuet-Gordon, H. (ed.) Karnak X, Le Trésor de Thoutmosis Ier, La céramique, Le Caire.

Holthoer, R.

1977 New Kingdom Pharaonic Sites. The Pottery, SJE 5:2, Lund.

Hope, C.

1987a Innovation in the Decoration of Ceramics in the Mid18th Dynasty, Cahiers de la Céramique Égyptienne 1, 97-122. 1987b Pottery of the Egyptian New Kingdom: Three Studies, Victoria College Archaeology Reasearch Unit, Occasional Paper No. 2, Burwood.

Hubschmann, C.

2010 Beer Jars of Mut el-Kharab, Dakhla Oasis: Evidence of Votive Activity in the Third Intermediate Period, Bulletin of the Australian Centre for Egyptology 21, 55-70.

JACQUET-GORDON, H.

1981 A Tentative Typology of Egyptian Bread Moulds, 11-24 in: Arnold, D. (ed.) Studien zur altägyptischen Keramik, SDAIK, Mainz am Rhein.

KLOTZ, D.

2013 The Earliest Representation of a Potter's Kick-Wheel in Egypt, Égypte Nilotique et Méditerranéenne 6, 169-176.

Loyrette, A.-M./NAsr, M./Bassiouni, S.

1993 Une tombe en bordure des greniers nord du Ramesseum, Memnomia 4, 115-127.

Mace, A. C./WinLock, H. E.

1916 The Tomb of Senebtisi at Lisht, Publications of the Metropolitan Museum of Art Egyptian Expedition, New York.

MASSON, A.

2011 Jarres au décor polychrome du Musée Pouchkine: manifestations originales de la tendance archaisante de 25e-26e dynasties?, 645-677 in: Aston, D. A./BADER, B./Gallorini, C./Nicholson, P./Buckingham, S. (eds.), Under the Potter's Tree, Studies on Ancient Egypt Presented to Janine Bourriau on the Occasion of her 70th Birthday, Orientalia Lovaniensia Analecta 204, Leuven, Paris, Walpole, CT.

Mensan, R.

2007 Tuthmosid Foundation Deposits at Karnak, Egyptian Archaeology 30, 21-25.

MYŚLIwIEC, K.

1987 Keramik und Kleinfunde aus der Grabung im Tempel Sethos' I. in Gurna, Archäologische Veröffentlichungen 57, Deutsches Archäologisches Institut, Abteilung Kairo, Mainz am Rhein.

Nelson, M.

2003 The Ramesseum Necropolis, 88-94 in: STRUdwick, N./Taylor, J. (eds.), The Theban Necropolis. Past, Present and Future, London.

NeLson, M.

2013 Une sépulture de la deuxième période intermédiaire (XVIIe) dynastie es ses ramifications, Memnonia 24, 91-103.

Nelson, M./Kalos, M.

2000 Concessions funéraires du Moyen Empire découvertes au nord-ouest du Ramesseum, Memnonia 11, $131-151$. 
Nordström, H./Bourriau, J.

1993 Fascicle 2. Ceramic Technology: Clays and Fabrics, 149-190 in: Arnold, D./Bourriau, J. (eds.), An Introduction to Ancient Egyptian Pottery, SDAIK 17, Mainz am Rhein.

Orton, C./Tyers, P./Vince, A.

1993 Pottery in Archaeology, Cambridge Manuals in Archaeology, Cambridge.

Petrie, W. M. F.

1909 Qurneh, BSAE 16, London.

Polz, D./Mählitz, E./Rummel, U./Seiler, A.

2003 Bericht über die 9. bis 12. Grabungskampagne in der Nekropole von Dra Abu el-Naga/Theben West, MDAIK 59, 317-388.

Pyke, G.

2005 Late Roman Egyptian Amphorae from Squares U and $\mathrm{V}$ at Kom el-Nana, 213-243 in: Faiers, J. (ed.) Late Roman Pottery at Amarna and Related Studies, London.

Quibell, J. E.

1898 The Ramesseum, London.

RiCKE, $\mathrm{H}$.

1939 Der Totentempel Thutmoses’ III, Beiträge zur Ägyptischen Bauforshung and Altertumskunde, Glückstadt-Hamburg.

Rzeuska, T.

2001 Pottery from the Temple of Tuthmosis III at Deir elBahari, Études et Traveaux 19, 300-328.

SÄve-SöDerbergh, T./Troy, L.

1991 New Kingdom Pharaonic Sites. The Finds and the Sites (Text), SJE 5, Uppsala.

Schiestl, R./Seiler, A.

2012 A Handbook of Middle Kingdom Pottery. Volume I: The Corpus Volume, Contributions to the Chronology of the Eastern Mediterranean 31, Vienna.

Seco Álvarez, M.

2015 Latest News about the Work of Investigation in the Temple of Millions of Years of the Pharaoh Thutmose III in Qurna, 101-133 in: Seco Álvarez, M./Jódar Minarro, A. (eds.), The Temples of Millions of years in Thebes, Granada.

Seco Álvarez, M./el Bialy, M./abd el Gafhar, N./Martinez Babon, J./Noureddine, I./Wilson, E./Guio, A./Gamarra Campuzano, A./Moreno Cifuentes, M.-A./Bader, B./AbD el Kerim, F. Y./el Nouby, E./AbD el Ghani Abd el Rahman/Amin, A./ Moнamed, F. K.

in press Fourth and Fifth Excavation Season of the EgyptianSpanish Project at the Mortuary Temple of Thutmosis III at the West Bank of Luxor (2011 and 2012), ASAE 87.
Seco Álvarez, M./Martinez Babon, J.

2015a A Foundation Deposit in the Temple of Millions of Years of Thutmose III in Luxor, Memnonia 25, 157167.

2015b Jewelry found in a 12th Dynasty Tomb at Luxor, Kmt 26 (2), 35-41.

2015c Middle Kingdom Tombs beneath the Temple of Millions of Years, Egyptian Archaeology 47, 27-30.

2015d A Ramesside Building in the Temple of Millions of Years of Thutmose III in Luxor, Studien zur Altägyptischen Kultur 44, 383-391.

Seco Álvarez, M./Radwan, A./Martinez Babon, J./Alaeldine, A./Kamimura, E./Mora Ruedas, E./Moreno Cifuentes, M.-A./ Noureddine, I./Perzlmeier, C./Abu el-Yazid, M. A./Abd El Kerim, F. Y./Abu Zaid, O./Mohamed, F. K.

2010 First Season of the Egyptian-Spanish Project at the Funerary Temple of Thutmosis III in Luxor, ASAE 84, $27-61$.

Seco Álvarez, M./Radwan, A./Martinez Babon, J./Noureddine, I./Wilson, E./Guio, A./Kamimura, E./Gamarra Campuzano, A./Mora Ruedas, E./Moreno Cifuentes, M.-A./Bader, B./Pazos, F./Abd el Kerim, F. Y./el Nouby, E./Mohamed, F. K.

2012/13 (appeared 2016) Second and Third Excavation Seasons of the Egyptian-Spanish Project at the Mortuary Temple of Thutmosis III at the West Bank of Luxor (2009 and 2010), ASAE 86, 329-395.

Seidlmayer, S.

1990 Gräberfelder aus dem Übergang vom Alten zum Mittleren Reich, Studien zur Archäologie und Geschichte Altägyptens 1, Heidelberg.

SeIDLMAYer, S.

2005 Regionale und chronologische Charakteristika der Beigabenkeramik des Friedhofs von Elephantine, 279-299 in: Pantalacci, L./Berger-el-Naggar, C. (eds.), Des Néferkare aus Montouhotep. Travaux archéologiques en cours sur la fin de la VI dynastie et la Première Période Intermédiaire, Lyon.

SeILER, A.

2005 Tradition \& Wandel. Die Keramik als Spiegel der Kulturentwicklung Thebens in der Zweiten Zwischenzeit, SDAIK 32, Mainz am Rhein.

2010 The Second Intermediate Period in Thebes: Regionalism in Pottery Development and its Cultural Implications, 39-53 in: MArÉE, M. (ed.) The Second Intermediate Period (Thirteenth -Seventeenth Dynasties, Current Research, Future Prospects, Leuven, Paris, Walpole, CT.

2012 Middle Kingdom Pottery in the Theban Necropolis, 299-320 in: Schiestl, R./Seiler, A. (eds.), Handbook of Pottery of the Middle Kingdom. Volume II: The Regional Volume, Contributions to the Chronology of the Eastern Mediterranean 31, Vienna. 
Sesana, A./Consonni, A.

2013 Une sépulture de nouveau-né dans l'aire du temple de millions d'années d'Amenhotep II à Thèbes-Ouest, Memnonia 24, 185-198.

SZAFRANSKI, Z.

1997 Foundation Pottery Deposit at the Djeser-akhet Platform, 53-64 Warsaw Egyptological Studies I Essays in Honour of Prof. Dr. Jadwiga Lipinska, Warsaw.
Weigall, A. E. P.

1906 Report on the Excavation of the Mortuary Temple of Thoutmose III in Gurneh, ASAE 7, 121-141.

1907 Note additionelle: Plan of the Mortuary Temple of Thoutmose III, ASAE 8, 286.

Weinstein, J.

1973 Foundation Deposits in Ancient Egypt, PhD, University of Pennsylvania. 